\title{
Considerations of Efficiency and Distributive Justice in Multidimensional Poverty Measurement
}

\author{
Dissertation \\ zur Erlangung des Doktorgrades \\ der Wirtschaftswissenschaftlichen Fakultät \\ der Georg-August-Universität Göttingen \\ vorgelegt von \\ Nicole Isabell Rippin \\ geboren in Bonn, Bad-Godesberg \\ Göttingen 2013
}

Gutachter:

Prof. Stephan Klasen, Ph.D.

Universität Göttingen

J-Prof. Dr. Sebastian Vollmer

Universität Göttingen 

To my wonderful family. I love you. 


\section{Eidesstattliche Versicherung}

1. Die Gelegenheit zum vorliegenden Promotionsvorhaben ist mir nicht kommerziell vermittelt worden. Insbesondere habe ich keine Organisation eingeschaltet, die gegen Entgelt Betreuerinnen und Betreuer für die Anfertigung von Dissertationen sucht oder die mir obliegenden Pflichten hinsichtlich der Prüfungsleistungen für mich ganz oder teilweise erledigt.

2. Ich versichere, dass ich die eingereichte Dissertation "Considerations of Efficiency and Distributive Justice in Multidimensional Poverty Measurement" selbstständig und ohne unerlaubte Hilfsmittel verfasst habe; fremde Hilfe habe ich dazu weder unentgeltlich noch entgeltlich entgegengenommen und werde dies auch zukünftig so halten. Anderer als der von mir angegebenen Hilfsmittel und Schriften habe ich mich nicht bedient. Alle wörtlich oder sinngemäß den Schriften anderer Autoren entnommenen Stellen habe ich kenntlich gemacht.

3. Die Richtlinien zur Sicherung der guten wissenschaftlichen Praxis an der Universität Göttingen werden von mir beachtet.

4. Eine entsprechende Promotion wurde an keiner anderen Hochschule im In- oder Ausland beantragt; die eingereichte Dissertation oder Teile von ihr wurden nicht für ein anderes Promotionsvorhaben verwendet.

5. Des Weiteren ist mir bekannt, dass Unwahrhaftigkeiten hinsichtlich der vorstehenden Erklärung die Zulassung zur Promotion ausschließen bzw. später zum Verfahrensabbruch oder zur Rücknahme des erlangten Titels berechtigen.

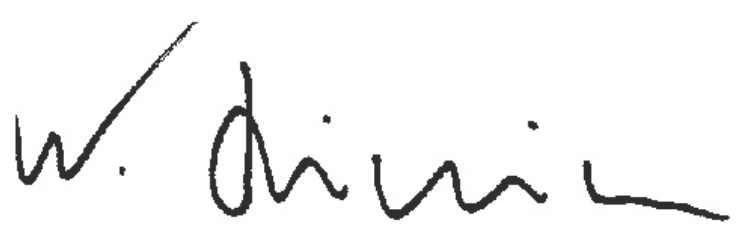

Nicole Isabell Rippin 


\section{Preface}

The twentieth century brought a lot of change for welfare economics that resulted in a profound transformation of poverty measurement. It began in the nineteen-thirties and -fifties when two waves of severe criticism concerning the comparability of individual utilities posed a serious threat to the long history of utility-based welfarism. For a transitional period the income approach moved into the centre of traditional theory. Though mostly considered as a mere placeholder until a new theory of welfare economics would take over, it should become the main approach to poverty measurement.

The situation changed with the development of the capability approach from the nineteen-eighties onwards; the first approach that actually had the potential to establish a new theory of welfare economics. The new approach made capabilities and functionings, i.e. what persons are actually able to do and be, the subject of analyses rather than economic resources or utility. Poverty under this approach is the failure to achieve a minimum set of central capabilities needed in order to be able to pursue whatever one has reason to value in life. This definition already indicates the approach's inherent respect of individual freedom and responsibility that makes it especially intriguing. The latter, however, becomes most obvious in the case of inequality.

The objective of distributive justice within the framework of the capability approach is to remove inequality of opportunity in order to create a level playing field that enables every individual to pursue whatever he or she has reason to value. Inequality of choice or effort is explicitly excluded. The clear distinction between the two types of inequality acknowledges the tension between distributive justice and efficiency, the objective of the latter being the expansion of the overall capability set available in a society. This distinction, though crucial, is seldom made in welfare economic theory.

Despite all its strengths, the capability approach faced a lot of resistance, mainly due to the fact that it replaces the subject of analysis, utility or income, with a set of subjects that are all measured in different units. Thus, a lot of scepticism was raised with regard to its operationalisability which obviously required a multidimensional approach. However, the initial resistance weakened over time. By now, new multidimensional indices are proposed on 
an almost daily basis. Interestingly, the new multidimensional approach is not only utilised to operationalise the capability approach, but also traditional welfarism. It seems that due to the sudden availability of the new multidimensional measures the latter experiences a rather unexpected resurrection.

The main objective of this volume is to analyse in how far the decision to define and measure poverty in terms of one of the three main theories of welfare economics that define well-being either as i) happiness (traditional welfarism), ii) opulence (income or expenditure approach), or iii) capabilities and functionings (capability approach) affects the empirical evaluation of poverty and poverty trends.

However, in order to be able to do so, a methodological weakness inherent in the current multidimensional approach to poverty measurement has to be addressed first. As mentioned before, one of the main strengths of the capability approach is that it explicitly accounts for the tension between the two concepts of distributive justice and efficiency (Sen, 1992, pp. 78):
'But equality is not the only social charge with which we have to be concerned, and there are demands of efficiency as well. An attempt to achieve equality of capabilities - without taking note of aggregative considerations - can lead to severe curtailment of the capabilities that people can altogether have. [...] Indeed, it will be argued that the import [sic!] of the concept of equality cannot even be adequately understood without paying simultaneous attention also to aggregative consideration - to the 'efficiency aspect' [...].'

One could easily consider distributive changes that are just but not efficient and vice versa. ${ }^{1}$ Nevertheless, in the current approach to multidimensional poverty measurement, this crucial difference is not made: inequality between poverty dimensions is generally treated as association sensitivity, thus reducing the concept of distributive justice to a mere analysis of how efficient poverty deprivations are distributed among the poor.

The first two essays of this volume are dedicated to overcome this methodological weakness. Starting point is the suggestion to define inequality between dimensions more

\footnotetext{
${ }^{1}$ As a simple example consider the distribution of six pairs of shoes between two persons. Initially, person 1 has five right shoes and one left shoe, whereas person 2 has one right shoe and five left shoes. Since left and right shoes are perfect complements, both persons are able to wear exactly one pair of shoes. This situation satisfies the concept of distributive justice. However, it is obvious that it is far from being efficient: through a simple switch of shoes both person could have three pairs each - a situation that still satisfies the concept of distributive justice. On the other hand, a switch of shoes that leaves person 1 with one right and one left shoe and person 2 with five right and five left shoes would satisfy the concept of efficiency but would violate the concept of distributive justice.
} 
holistically as the association-sensitive spread of simultaneous deprivations across the population.

The first essay deals with the operationalisation of the extended definition of inequality between dimensions in the case of ordinal poverty indices. It introduces a new property called "Sensitivity to Inequality Increasing Switch (SIIS)" that conditions the extent to which an inequality increasing switch increases poverty on the relationship between poverty dimensions. The new property is utilised to derive a new class of multidimensional poverty indices that is unique in the sense that it is the first class of additive poverty indices that is able to account for inequality as well as association-sensitivity.

The second essay does essentially the same with regard to cardinal poverty indices. It introduces a new property called "Inequality Sensitivity (IS)" that basically requires poverty to increase (in the case of substitutes) or to decrease (in the case of complements) if an association increasing switch between two poor individuals comes at the expense of the poorer of the two. Again, the new property is utilised to derive a new and unique class of multidimensional indices that, though additive, is able to account for both inequality within and between poverty dimensions on one hand and the relationship between poverty dimensions on the other.

Once the methodological weakness has been corrected, the third essay uses the rich data source of the German Socio-Economic Panel in order to propose a new (ordinal) multidimensional poverty index for Germany that is based on the capability approach, the so called German Correlation Sensitive Poverty Index. It also introduces a multidimensional happiness index, the Subjective Correlation Sensitive Poverty Index, that is based on traditional welfarism and is derived from a satisfaction-based self-evaluation of different poverty dimensions. Finally, the official poverty measure of Germany, i.e. the at-risk-ofpoverty rate, defined as 60 per cent of the median net equivalence income, is calculated as a representative of the income approach. All three indices are compared across dimensions, regions and over time, and the results seem to indicate one thing above all: the significant differences in the evaluation of poverty and poverty trends induced by the different indices and the high added value that is created through the operationalisation of the capability approach. 


\section{Acknowledgements}

This thesis would not have been possible without the support of many persons who contributed in one way or another to its completion.

First and foremost, I want to thank my supervisor Professor Stephan Klasen for giving me the opportunity to write this thesis as an external $\mathrm{PhD}$ student. I am well aware that this is something not many professors would be willing to do as it means a lot of work while getting little in return. I decided to approach Stephan Klasen because of what I knew about his excellent scientific reputation but I got much more than I expected. It never ceases to amaze me how he skilfully managed to give me all freedom to do my own research and follow my ideas while at the same time providing the best possible support whenever needed. I am more than grateful.

As well, I would like to express my deepest thanks to my second supervisor Junior Professor Sebastian Vollmer for carefully reading and commenting on several revisions of my writings. This thesis benefited greatly from his insightful comments and constructive criticism, and his practical advice and kind encouragement were a great support especially at the final stage of the work.

My heartfelt thanks also go to my colleagues at the German Development Institute, especially Tilman Altenburg and Markus Loewe, for understandingly releasing me from tedious tasks and for patiently enduring me in stressed mood. I could not imagine a better working atmosphere.

I am also very thankful to Sabina Alkire, Eva Jespersen, Stephan Klasen, and Peter Krause for facilitating my research stays at the Oxford Poverty and Human Development Initiative, the Human Development Report Office, the University of Göttingen, and the German Institute for Economic Research, respectively, as well as, of course, their whole staff. My research benefited greatly from the deep knowledge that they readily shared and their openness and friendliness made the resarch stays an experience that I will always treasure.

I also wish to thank the many scientists who helped me with their valuable comments and suggestions all along the way, in particular Valérie Bérenger, Florent Bresson, Satya Chakravarty, Conchita D’Ambrosio, Markus Grabka, Ortrud Leßmann, Milorad Kovacevic, 
Jacques Silber, Subbu Subramanian, Suman Seth, Maria Emma Santos, Casilda Lasso de la Vega, Gaston Yalonetzky and several anonymous referees.

In addition, I would like to thank the participants of the following conferences for their helpful comments and suggestions: the Human Development and Capability Association's annual conference (Amman, 2010; The Hague, 2011; Jakarta, 2012), the Fourth Meeting of the Society for the Study of Economic Inequality (Catania, 2011), the OECD Universities' Joint Economics Congress: New Directions in Welfare II (Paris, 2011), the Research Committee of the German Economic Association on Development Economics' annual conference (Bonn, 2012), and the $32^{\text {nd }}$ International Association for Research in Income and Wealth conference (Boston, 2012).

The financial assistance of the German Federal Ministry for Economic Cooperation and Development is gratefully acknowledged.

Last but in no way least, I am most grateful to all my friends, in particular Mario, Andreas, Julia, Jimmy and Josefa, whose readiness to listen was a great support especially during the hard times. I also want to thank my wonderful family, in particular my grandparents and parents, who always believed in me and continuously encouraged me to reach higher. Finally, my deepest thanks go to my husband whose love gave me stability and carried me through times of crisis. Without his continuous support I could not have done any of this. 


\section{Contents}

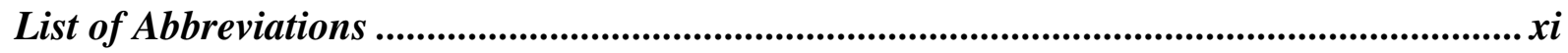

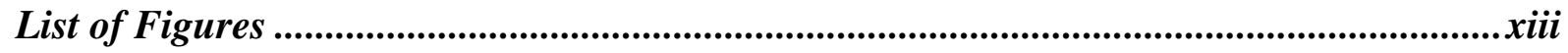

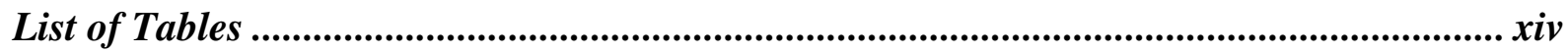

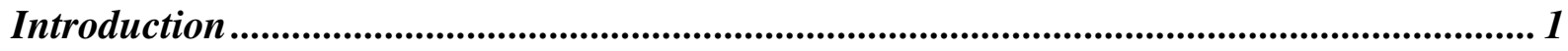

1 Efficiency and Distributive Justice in Ordinal Poverty Indices .................................... 13

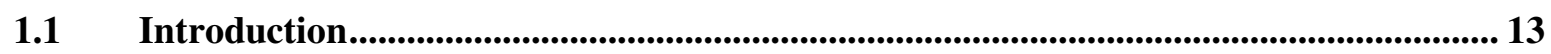

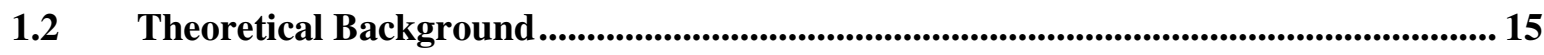

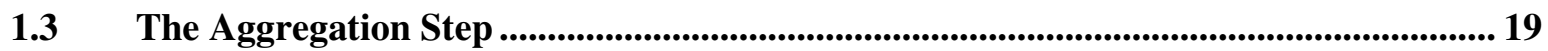

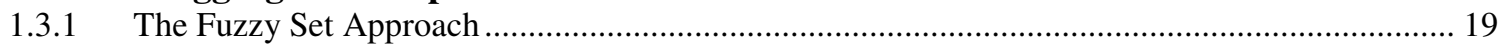

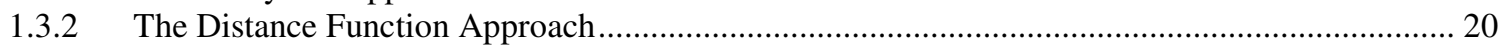

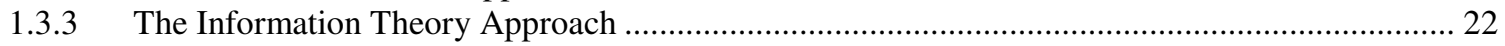

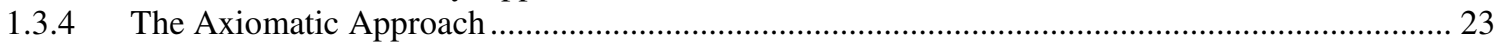

1.4 Correlation-Sensitive Poverty Indices...................................................................................... 27

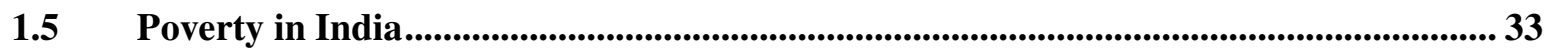

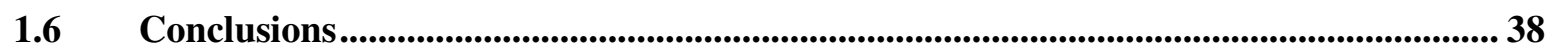

2 Efficiency and Distributive Justice in Cardinal Poverty Indices .................................. 40

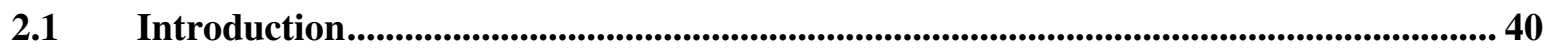

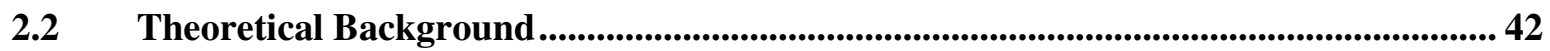

2.3 The Axiomatic Foundation for Cardinal Poverty Measures............................................. 45

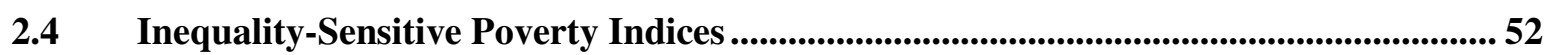

$2.5 \quad$ Empirical Application .................................................................................................................. 58

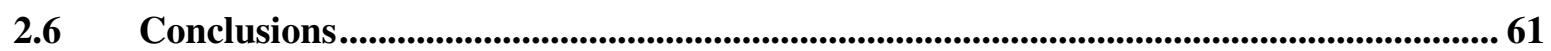

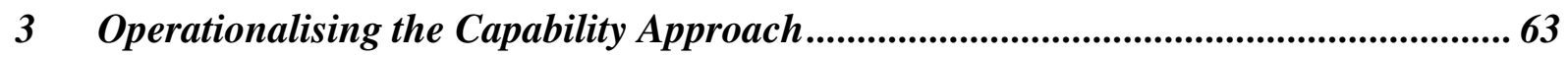

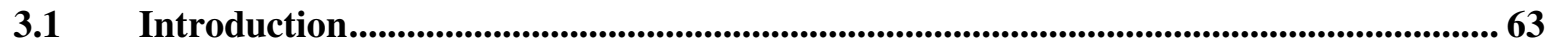

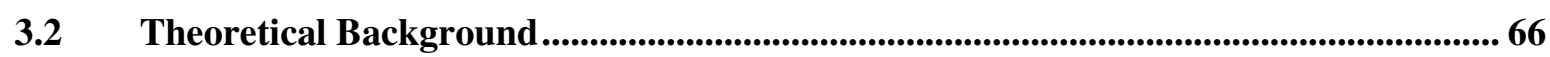

3.3 The Axiomatic Foundation and Decomposition ..........................................................68

3.4 The German Correlation Sensitive Poverty Index........................................................ 71

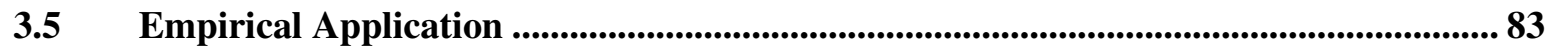

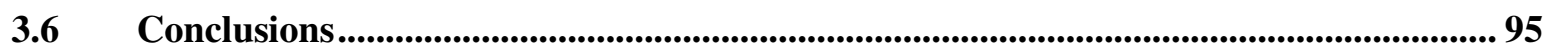

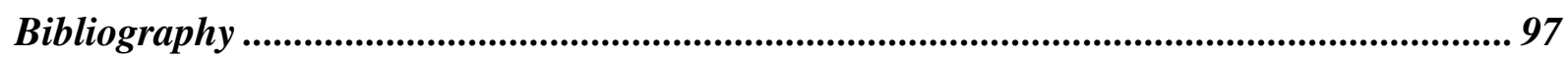

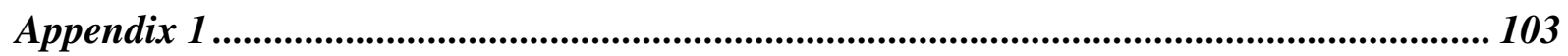

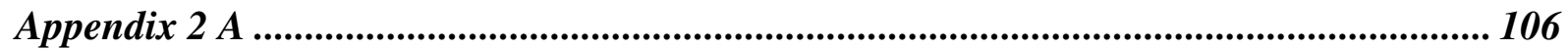

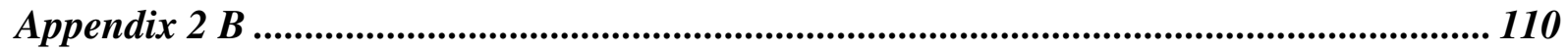

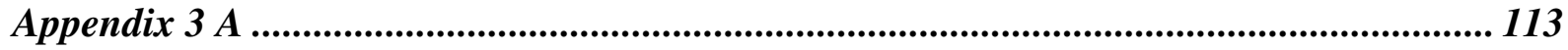

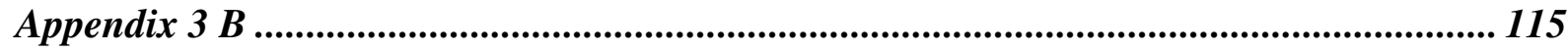




\section{List of Abbreviations}

\begin{tabular}{|c|c|}
\hline ALEP & Auspitz-Lieben-Edgeworth-Pareto \\
\hline $\mathbf{A N}$ & Anonymity \\
\hline BMI & Body Mass Index \\
\hline CSPI & Correlation Sensitive Poverty Index \\
\hline CLI & Composite Living Index \\
\hline DIE & German Development Institute \\
\hline DIW & German Institute for Economic Research \\
\hline DHS & Demographic and Health Surveys \\
\hline EPC & Equality-Promoting Change \\
\hline FD & Factor Decomposability \\
\hline FGT & Foster-Greer-Thorbecke \\
\hline GCSPI & German Correlation Sensitive Poverty Index \\
\hline GE & Generalized Entropy \\
\hline GSOEP & German Socio-Economic Panel \\
\hline IS & Inequality Sensitivity \\
\hline $\mathbf{H}$ & Headcount \\
\hline ISPI & Inequality Sensitive Poverty Index \\
\hline IT & Information Technology \\
\hline MDG & Millennium Development Goals \\
\hline MPI & Multidimensional Poverty Index \\
\hline $\mathbf{M N}$ & Monotonicity \\
\hline NDA & Nondecreasingness under Association Increasing Switch \\
\hline NIA & Nonincreasingness under Association Increasing Switch \\
\hline NIPA & Nonincreasingness under Pareto-efficient Association Increasing Switch \\
\hline NM & Normalization \\
\hline OPHI & Oxford Poverty and Human Development Initiative \\
\hline $\mathbf{P P}$ & Principle of Population \\
\hline SCSPI & Subjective Correlation Sensitive Poverty Index \\
\hline SD & Subgroup Decomposability \\
\hline
\end{tabular}


SF Strong Focus

SI Scale Invariance

SIIS Sensitivity to Inequality Increasing Switch

UM Uniform Majorization

UNDP United Nations Development Programme

USAID U.S. Agency for International Development

WAI Weighted Asset Index

WHO World Health Organization 


\section{List of Figures}

Figure 0.01 Transforming Economic Resources into Utility ............................................................. 4

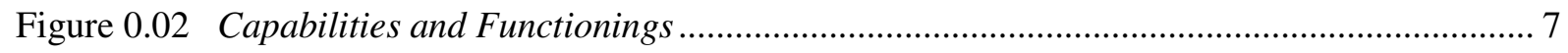

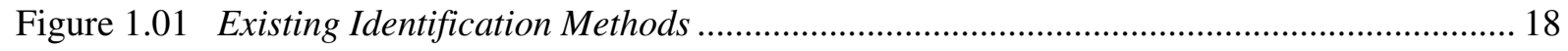

Figure 1.02 The Correlation Sensitive Identification Method .......................................................... 28

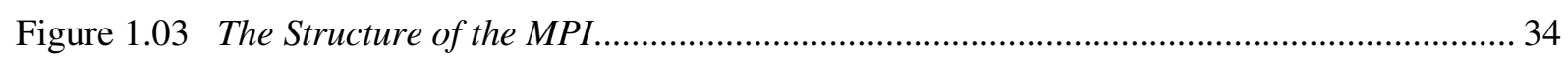

Figure 1.04 Indian Poverty Maps according to MPI and CSPI ...................................................... 38

Figure 2.01 The Correlation Sensitive Identification Method ............................................................ 44

Figure 2.02 Pareto-Efficiency and Association Increasing Switches ................................................. 49

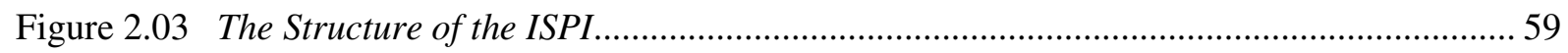

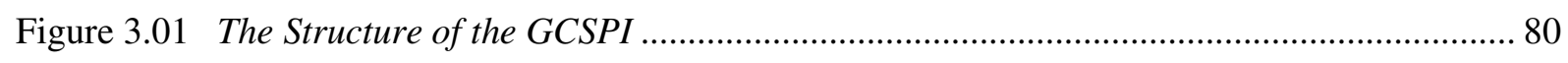

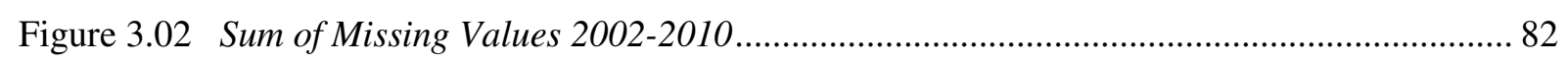

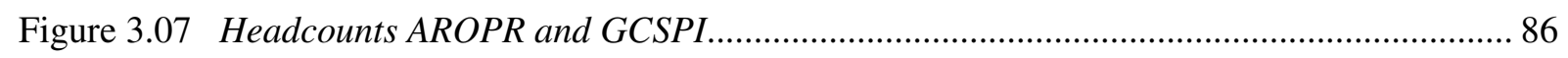

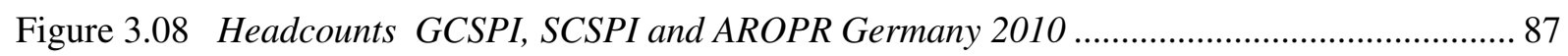

Figure 3.09 Development of Economic Figures Germany 2002-2010 ............................................. 88

Figure 3.10 Different Poverty Paths in Germany 2002-2010 ........................................................ 90

Figure 3.11 German Poverty Maps AROPR 2002-2010 ……......................................................... 91

Figure 3.12 German Poverty Maps GCSPI 2002-2010 .................................................................. 92

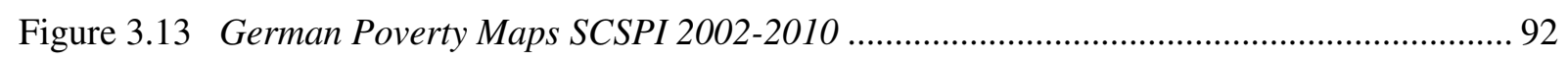

Figure 3.14 Development of Dimensional Decompositions 2002-2010 …......................................... 93

Figure 3.15 Dimensional Decomposition of GCSPI 2010................................................................ 94

Figure 3.03 Frequency Distribution Satisfaction with Health ....................................................... 113

Figure 3.04 Frequency Distribution Satisfaction with Work .......................................................... 113

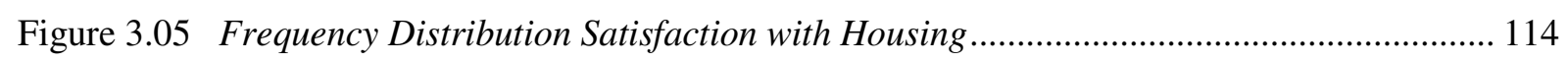

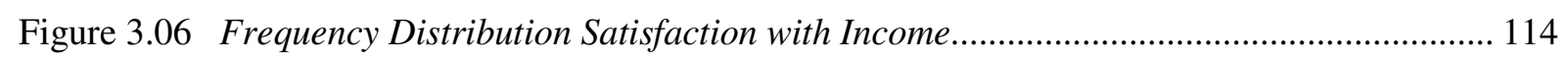




\section{List of Tables}

Table 1.01 Axiomatic Foundation of Selected Ordinal Poverty Measures ........................................ 31

Table 1.02 A Comparison of Five Indian Households (DHS 2005) ................................................ 34

Table 1.03 Poverty Rates and Decompositions Selected Indian States............................................. 35

Table 1.04 The CSPI and Selected Decompositions for India (DHS 2005) ...................................... 36

Table 2.01 Axiomatic Foundation of Selected Cardinal Poverty Measures ....................................... 55

Table 3.01 Spearman Rank Correlation Equal and Prevalence Weights ......................................... 82

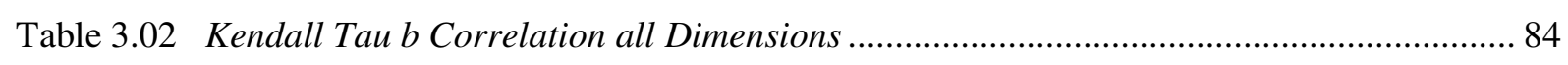

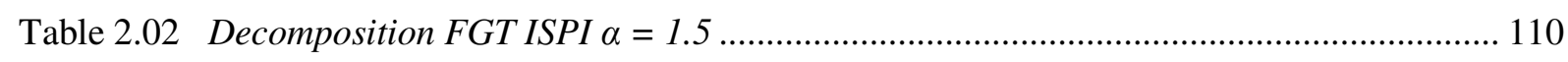

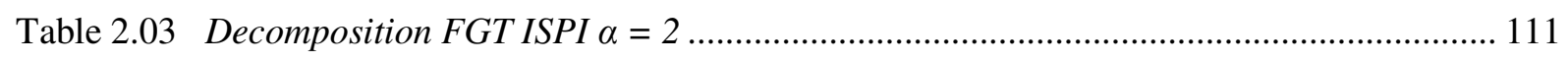

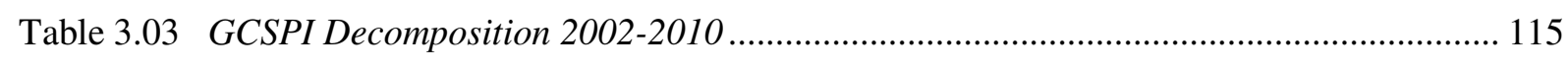

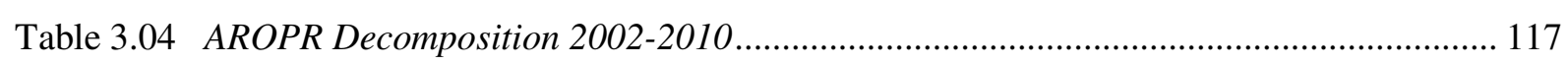

Table 3.05 GCSPI Decomposition 2002 (prevalence weights) ...................................................... 117

Table 3.06 GCSPI Decomposition 2004 (prevalence weights) ...................................................... 118

Table 3.07 GCSPI Decomposition 2006 (prevalence weights) ...................................................... 118

Table 3.08 GCSPI Decomposition 2008 (prevalence weights) ...................................................... 119

Table 3.09 GCSPI Decomposition 2010 (prevalence weights) ..................................................... 119

Table 3.10 GCSPI Decomposition 2002 (equal weights)............................................................. 120

Table 3.11 GCSPI Decomposition 2004 (equal weights)............................................................... 120

Table 3.12 GCSPI Decomposition 2006 (equal weights).............................................................. 121

Table 3.13 GCSPI Decomposition 2008 (equal weights)............................................................ 121

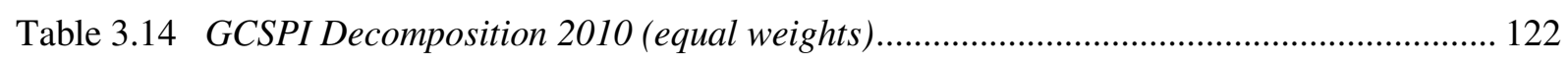

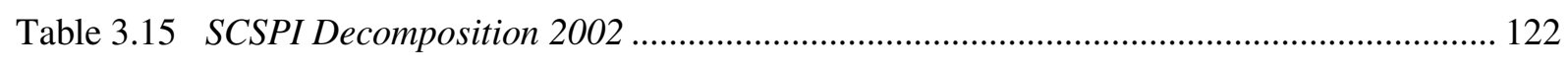

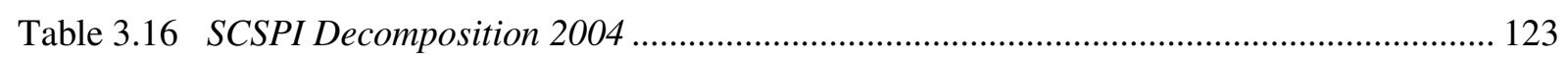

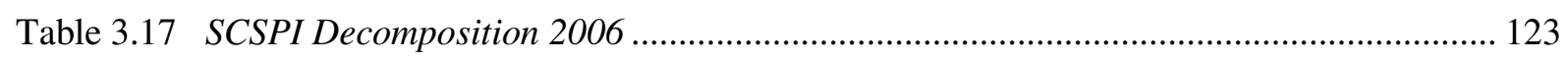

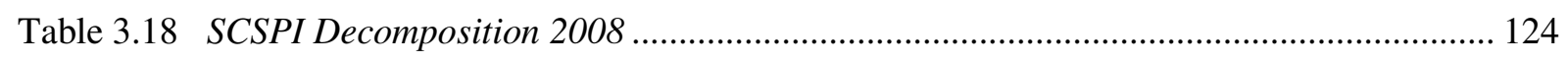

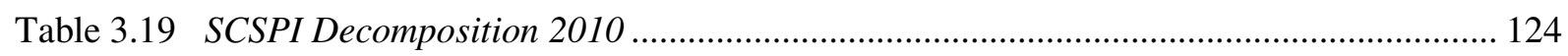




\section{Introduction}

'[W]ealth is evidently not the good we are seeking; for it is merely useful and for the sake of something else'

Aristotle in Nicomachean Ethics (Volume 1, p. 7)

Welfare economics, which according to Amartya Sen 'is the part of economics that is concerned with the assessment of the goodness of states of affairs and the appraisal of policies' (Sen, 2009, p. 272), has traditionally equated well-being with happiness, placing the latter at the centre of analysis. The roots of this tradition can be traced back as far as the fourth century BC, to the time of Aristotle. In his two-part 'philosophy of human affairs', the Greek philosopher argues that happiness, or Eudaimonia, is the final human good, the one thing every person is striving to achieve (Bartlett and Collins, 2012, p. x). His approach would influence economics for centuries to come, such as the theory of utilitarianism, which typically defines utility as some psychological metric such as happiness or pleasure.

\section{Traditional Welfarism}

Utilitarianism, pioneered in 1789 by Jeremy Bentham (Bentham, 2007), continued to provide the basis for welfare economics until well into the nineteen-thirties: traditional welfarism measured the goodness of states of affairs in a society as the sum of individual utilities in that society, thereby building upon the cardinal measurement, comparison and aggregation of individual utilities. It is not hard to see that this is a very ambitious approach, especially considering the fact that utility is a rather fuzzy concept that is already difficult to measure, let alone to compare and aggregate in a fairly reasonable manner.

In fact, it was the assumption of interpersonal comparability of utility that eventually dealt the first major blow to traditional welfare economics in the nineteen-thirties. Researchers such as the British economist Lionel Robbins argued that interpersonal comparisons of individual (cardinal) utilities cannot possibly be made in any sound scientific sense: 'I still cannot believe that it is helpful to speak as if interpersonal comparisons of utility 
rest upon scientific foundations - that is, upon observation or introspection. I am perhaps more alive than before to the extraordinary difficulties surrounding the whole philosophy of valuation' (Robbins, 1938, p.640).

At the heart of the problem is the fact that mental conditioning as well as adaptive preferences, whether upward or downward, can have a significant impact on individual utilities (Dworkin, 1981a, pp. 228-240; Sen, 1987, p. 11; Roemer, 1998, pp. 238-239). An example of the former is provided by "expensive tastes", which refer to the special preferences of an individual who needs more than others in order to reach the same utility level. Assuming equal capacity for satisfaction would disregard this issue - and consequently allocate a larger share of society's resources to this individual. Another example would be "offensive tastes", which denote the special, actually sadistic preferences of an individual who needs to have his or her fellow creatures treated poorly in order to reach the same utility level as others. An example of the latter is what has become known as the "tamed housewife" scenario, describing a situation in which continuously bad circumstances result in a downward adaptation of preferences, leading to a situation in which individuals need far less than others in order to achieve the same level of utility.

Despite the severe and not easily rejectable criticism, traditional welfarism managed to survive, though in the sharply reduced version of revealed preference welfarism, pioneered by Abram Bergson (1938) and Paul Samuelson (1948). Revealed preference welfarism dissociated itself from any kind of interpersonal (cardinal) utility measurement, comparison and aggregation. Instead, it relied solely on the (ordinal) observable expression of preferences. However, about ten years later, the slimmed down version of traditional welfarism received a second and this time final blow.

The blow came in the form of what was to become one of the most famous theorems, i.e. the impossibility theorem of Kenneth Arrow (1950). What Arrow demonstrated is that any policy decision based on revealed preferences with regard to three alternatives or more is unable to satisfy a set of very few reasonable properties. In particular, he introduced the following three axioms: 1 . Unrestricted Domain (U), i.e. the domain is defined for the set of all preference orders of the society; 2. Weak Pareto (WP), i.e. whenever all members of a society prefer alternative $x$ to $\mathrm{y}$, then $x$ should be collectively preferred to $y$ as well; 3 . Independence of Irrelevant Alternatives (I), i.e. whenever additional alternatives are offered but do not change any of the individual preference orders, the collective decision should not change either. 
Kenneth Arrow's impossibility theorem demonstrates that in the case of three alternatives or more, any decision rule that satisfies $\mathrm{U}, \mathrm{WP}$ and I is unable to satisfy another highly desirable property referred to as Non-Dictatorship (ND) which requires that collective decisions are not dictated by the preferences of one individual. This result, astonishing at first glance, is driven by the fact that the restriction on revealed preferences reduces any decisionmaking process to a voting method of some form or another. However, the exposure of voting methods to a series of consistency problems was recognised almost two hundred years earlier, for instance by the French mathematician and philosopher Marie Jean Antoine Nicolas Caritat, better known as the Marquis de Condorcet. In 1785, he made an observation that was to go down in history as the Condorcet Paradox and that illustrates vividly the consistency problems of voting methods.

Consider, for instance, the following very simple example of the Condorcet Paradox. Imagine a society with three individuals who have to decide between three different alternatives, i.e. $x, y$ and $z$. Furthermore, assume that the three individuals have the following preferences over $x, y$, and $z$ :

$\left.\begin{array}{ccc}\text { Ind.1 } & \text { Ind.2 } & \text { Ind.3 } \\ x & y & z \\ y & z & x \\ z & x & y\end{array}\right\} \begin{array}{ll}x \succ y: & 2 \\ y \succ z: & 2 \\ z \succ x: & 2\end{array}$

Given that the society as a whole prefers $x$ to $y(x \succ y)$ and $y$ to $z(y \succ z)$, one would logically expect that $x$ would be preferred to $z(x \succ z)$, a property that has become known as transitivity. However, in the example above, the society as a whole prefers $z$ to $x(z \succ x)$ so that any decision based on this society's voting is inevitably arbitrary and - dictatorial.

Numerous studies worked on rehabilitating revealed preference welfarism, but to no avail. Different versions of the impossibility theorem proved that it applied in one form or another to any social welfare function that was not of the type "cardinally fully comparable", i.e. the very type whose rejection led to the development of revealed preference welfarism in the first place. This opened the door for alternative approaches.

\section{The Income Approach}

Leaving aside those approaches that aimed to replace the whole idea of welfarism, for instance contractarian or libertarian approaches, one concept that is closely related to utilitarian welfare economics seemed to gain broad acceptance was the income approach, 
which can be traced back to William Petty (1623-1687), who is acknowledged as the pioneer of national income accounting. It is interesting to note that, as Amartya Sen points out (2009, p. 226), William Petty explicitly mentioned other determinants of individual condition besides income, such as happiness and safety. Likewise, the deliberate restriction that Cecil Pigou imposed when he suggested, as early as 1932, that national income be used to measure 'that part of social welfare, that can be brought directly or indirectly into relation with the measuring-rod of money' was largely ignored (Pigou, 2005, p.11).

It seems that after struggling for years with the problem of comparing utility, an easy approach was rather tempting. Concepts such as well-being, poverty, and inequality were almost exclusively measured in monetary terms over the next few decades.

However, the chain from economic resources to utility (whatever the definition ${ }^{2}$ ) is anything but straightforward.

Figure 0.01 Transforming Economic Resources into Utility

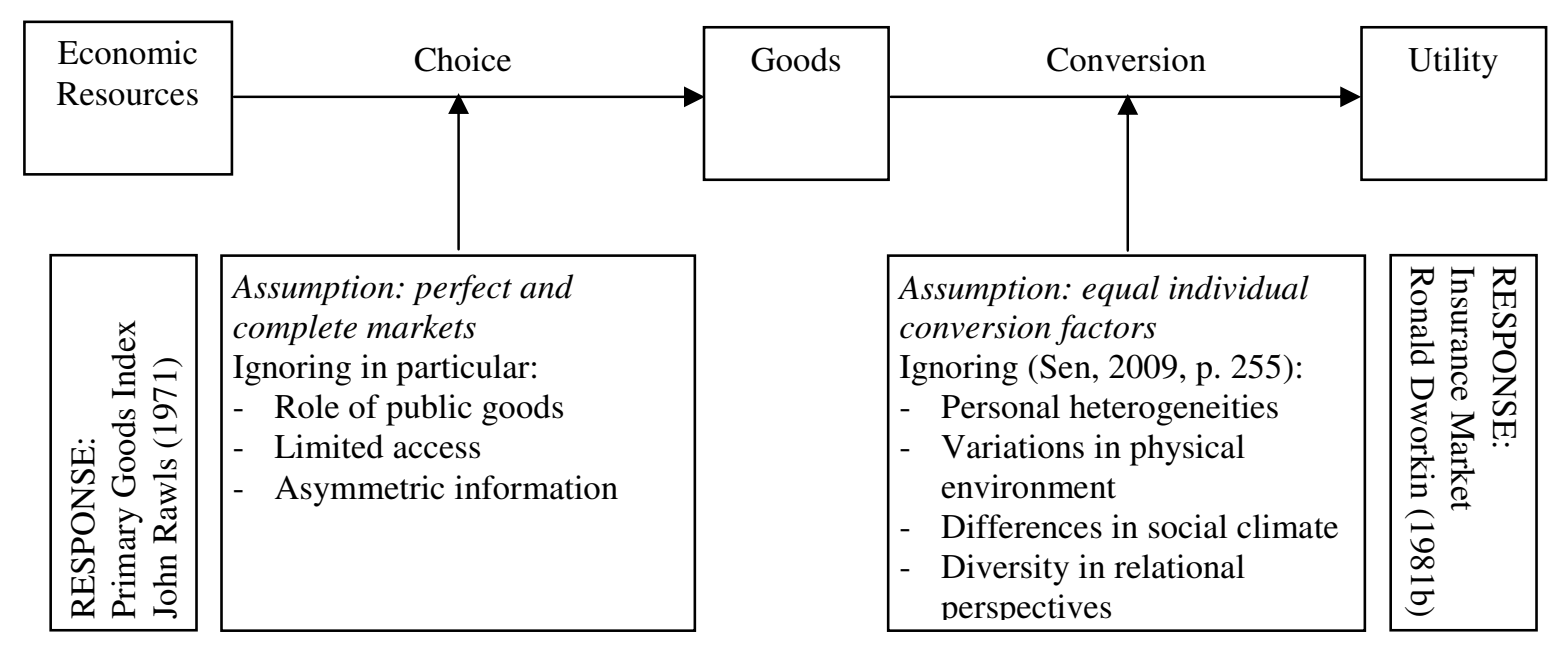

In response to the theoretical dead-end represented by a concentration on the outcome utility, there has been a shift towards the input economic resources (wealth, income) as the new basis for welfare economics. Aristotle's statement was rarely contested that economic resources were not an end in themselves but rather means to an end. Specifically, it was assumed that economic resources provided a sufficiently precise proxy for the actual subject matter, i.e. utility. In order for this proposition to hold, two assumptions must be made: that perfect and complete markets exist and that individual conversion factors do not vary, i.e. all

\footnotetext{
${ }^{2}$ In the following, I will define utility as "something that individuals value and have reason to value", whatever the individual definition of that something may be. Also, I will leave aside the whole question how utility functions might look like as this would go well beyond the scope of this thesis.
} 
individuals have the same ability to convert goods into utility. Over the course of time, however, the accuracy of these two assumptions has been increasingly questioned.

With regard to the perfect and complete markets assumption, it was stated that i) especially in developing countries many individuals only have limited access to markets; ii) markets for many of the most basic goods do not exist at all, for instance, public goods such as access to healthcare and education systems; and, finally, iii) due to asymmetric information, prices can neither be considered efficient nor fair. With regard to the assumption of individual conversion factors being equal, Amartya Sen (2009, p. 255) actually provided a whole list of factors influencing the ability of individuals to convert goods into what they have reason to value: i) personal heterogeneities, caused by any kind of handicap or any kind of adversity that individuals might face. A disabled person, for instance, will need more goods and thus more resources than a healthy person in order to live a "normal life"; ii) differences in the physical environment; iii) differences in the social environment; and, finally, iv) diversity in relational perspectives, which refers to a situation in which a person's absolute standing depends on his or her relative standing compared to other members of society. An example of the latter is provided by Adam Smith: the ability to appear in public without shame, which strongly depends on what Adam Smith calls 'established rules of decency' (Smith, 1776, p. 467).

Due to the justified criticism, many researchers have sought time and again to create alternative approaches for a new - and convincing - theory of welfare economics. In an effort to overcome the problems connected with the perfect and complete market assumption, John Rawls introduced in his theory of justice (1971) an index of primary goods which are defined as goods that 'a rational man wants whatever else he wants' (Rawls, 1971, p. 79). They are goods that every person needs to have in order to successfully realise his or her individual life plan. The index of primary goods is intended to replace economic resources as the object of analysis in his theory of (social) justice. Whereas Rawls' index successfully avoids the perfect and complete market assumption, it has some serious weaknesses of its own.

For one thing, it is by no means obvious what these primary goods are that all people want or who should define them. Rawls himself provides five broad categories: 'rights, liberties, and opportunities, and income and wealth' (Rawls, 1971, p. 79). It would be difficult to agree on even these broad categories in all cultures, but in order to apply the index in practice, a much more detailed definition would be needed. At the root of the problem is the fact that the removal of the complete and perfect market assumption led to the loss of another aspect, that of individual choice. The objective of the income approach is to ensure that 
persons receive a minimum amount of money. However, it is up to them how they actually spend their money. The primary goods approach, on the other hand, leaves no scope for individual choice as it predetermines the goods people ought to have. Even where there is overwhelming consensus on the selection of these primary goods, Rawls' approach still has a distinct taste of paternalism about it. In addition, it still suffers from the fact that the ability to convert goods into utility can vary greatly from individual to individual. However, any measurement approach that ignores these differences gives a misleading image of individual poverty and consequently leads to distribution policies that are, in fact, unjust (e.g. Sen, 2009, p. 255).

In an effort to overcome the weaknesses of Rawls' approach, Ronald Dworkin (1981b) developed the idea of a hypothetical insurance market. He draws on John Harsanyi's concept of 'choice involving risk' (1953, p. 435) in which the unborn soul has to choose between different types of income distribution in a society without knowing which social position it will be born into ${ }^{3}$. Dworkin's idea is that individuals, behind the veil of ignorance, have the opportunity to buy insurance against any kind of conversion handicap they might experience in their lives. Those who choose to insure themselves will receive compensation for their handicap as determined by the insurance they purchased. With such a hypothetical insurance market in place, so goes the argument of Dworkin, the problem of different conversion factors can be overcome and real equality of resources can be achieved. It should be noted that Ronald Dworkin differentiates between two kinds of inequality. His insurance market provides compensation for inequality that is due to external circumstances, while inequality that is due to individual choices and preferences is not compensated.

One of the main problems of Dworkin's approach, though, is that while it works rather well in the case of individual handicaps, it is much harder to consider insurance markets for non-personal characteristics (Sen, 2009, p. 266). In addition, his approach also relies on the same perfect and complete market assumption as the income approach.

\section{The Capability Approach}

Though both of the aforementioned approaches have become famous theories in social science, neither of them was convincing enough to successfully compete with the highly appreciated simplicity of the income approach. However, another approach has been

\footnotetext{
${ }^{3}$ This concept has become better known in economic literature by the term used by John Rawls in his theory of justice: the 'veil of ignorance' (Rawls, 1971, p. 118).
} 
developed that deliberately breaks away from traditional thinking: the capability approach developed by the Indian economist and philosopher Amartya Sen (1979, 1985, 1992, 1999, 2009). Each of the approaches presented so far focuses on the instruments for achieving wellbeing, a logical consequence following the abandonment of utilitarianism and its failure to make well-being itself the subject of analysis. The novelty of the capability approach is its introduction of so-called functionings and capabilities that are not instruments but constitute elements of well-being (Sen, 1992, p. 39).

Functionings are the "beings" and "doings" individuals are able to be and do (Sen, 1992, p. 39). Functionings are rather different to commodities. They constitute an aspect of living itself whereas commodities are objects a person might use. According to Sen, basic functionings include the ability to be well-nourished, to live a healthy life and to walk about without shame. Whereas a person's set of functionings represents his or her actual achievements, the set of achievable functioning vectors constitutes that person's capability set. In other words, a person's capability set represents his or her freedom to choose between different functioning bundles and thus that person's opportunities to achieve well-being.

Figure 0.02 Capabilities and Functionings

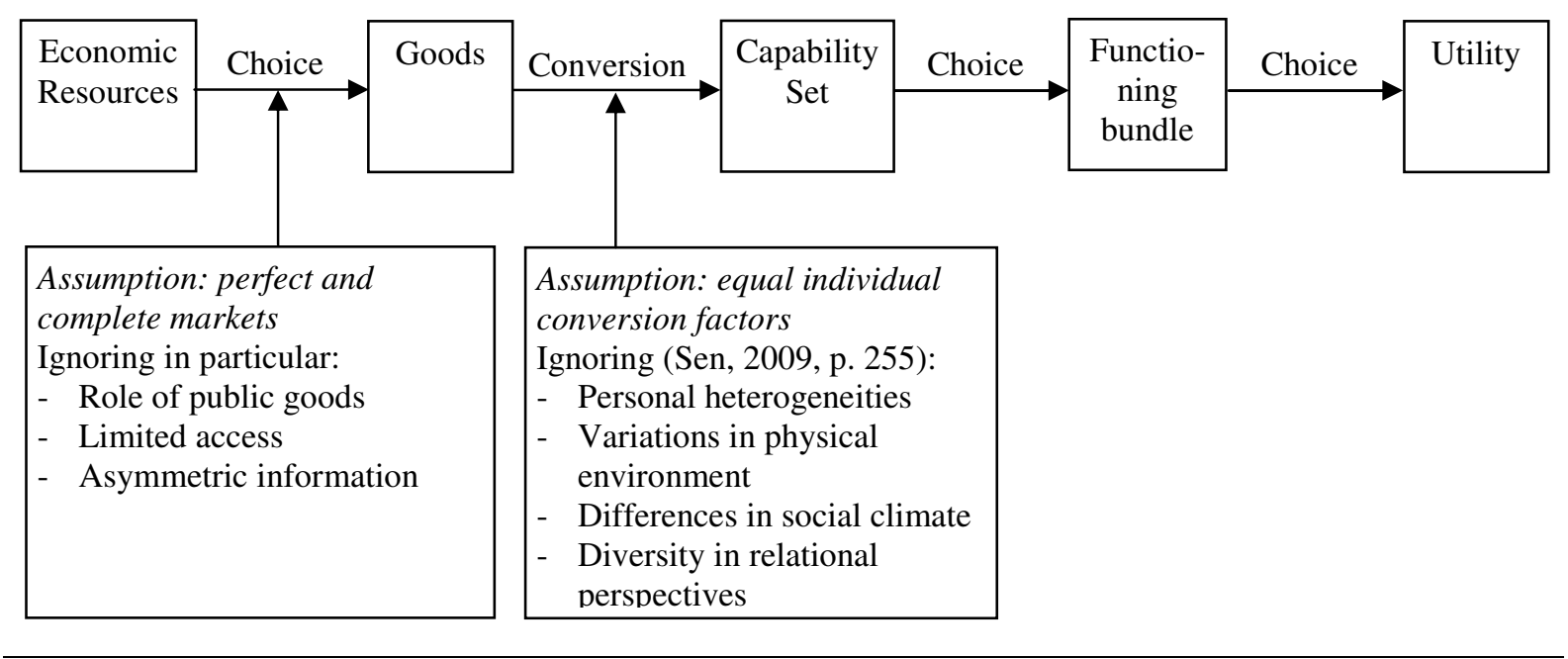

Through the introduction of capabilities and functionings, the capability approach successfully bypasses the problems associated with the assumptions of perfect and complete markets and of equal individual conversion factors while at the same time introducing two very interesting aspects of individual responsibility and freedom of choice.

The first aspect is introduced by the differentiation between the capability set of possible functioning bundles on one hand and the functioning bundles themselves on the other. Consider the following example of Amartya Sen (1992, p. 52): Imagine two individuals who are undernourished. One individual is undernourished because he or she is starving, i.e. lacks 
the means to satisfy his or her hunger. The other person, however, has enough means to satisfy his or her hunger, but deliberately chooses to fast for religious reasons. Standard theory would treat both individuals the same, however, the capability approach differentiates between the two as they have very different capability sets. While neither may meet a specific minimum calorie intake, the former has no choice but to starve due to a restricted capability set. The latter, on the other hand, has the capability to eat, but out of free choice refuses to do so.

The second aspect is introduced through the fact that though the individually chosen functioning bundles are constitutive elements of utility (or well-being), they can also be utilised to extend the capability set and thus enhance well-being. In other words, a sufficiently large initial capability set enables individuals to pursue well-being, but does not ensure wellbeing, as the latter is a matter of individual responsibility. It is interesting to note here that when Thomas Jefferson (1743-1826) penned the Declaration of Independence (ratified on the $4^{\text {th }}$ of July 1776), he seemed to have a similar idea in mind. Up until that point, the prevailing opinion of what rights a government must ensure could be traced back to the British philosopher John Locke (1634-1704), who, in his 'Second Treatise of Government' (1690), claimed these rights to be life, liberty and possessions ${ }^{4}$. Thomas Jefferson deliberately replaced the last aspect when he penned: 'We hold these truths to be self-evident, that all men are created equal, that they are endowed by their Creator with certain unalienable Rights, that among these are Life, Liberty and the pursuit of Happiness.' It is very interesting that he wrote of the rights to life and liberty, but not the right to happiness. Instead, he called for the right to pursue happiness, acknowledging that happiness itself is something closely bound up with individual responsibility and choice.

Despite the obvious and intriguing advantages of the capability approach, it has not been adopted readily. The reason is that, while undoubtedly intriguing, the theoretical framework of the capability approach is rather difficult to operationalise. For one thing, though the new approach introduces different aspects of choice, it neglects another one, the choice of those "essential capabilities" that shape the minimum capability set individuals ought to have in order to be able to pursue their goals in life. Sen rightfully claims that this weakness is not as bad as in the case of Rawls' primary goods approach, as it is much easier to agree on capabilities than on goods (e.g. Sen, 1985, p.6). For instance, it is much easier to agree on the

\footnotetext{
${ }^{4}$ 'The state of nature has a law of nature to govern it, which obliges every one: and reason, which is that law, teaches all mankind, who will but consult it, that being all equal and independent, no one ought to harm another in his life, health, liberty, or possessions [...].' (Locke, 1690, chapter 2, Sect. 6).
} 
capability to avoid undernourishment than it is to agree on a specific calorie intake. However, he also acknowledges that choosing the essential capabilities is still a difficult and controversial task. Consequently, Sen refused to provide even a list of broad categories that could be used to operationalise his approach. It was the American philosopher Martha Nussbaum who bridged this gap by suggesting ten 'central human capabilities' (Nussbaum 2003, pp. 41-42). Though her list is far from uncontested, it is already being widely used to apply the capability approach. The other reason for scepticism towards this approach is the plurality inherent in the nature of capabilities and functionings. Comparing, even aggregating, different functionings or capabilities has been reproached for adding 'apples and bananas'.

Yet, the capability approach has so many intriguing advantages, especially when it comes to measuring poverty and inequality, that it is beginning to gain traction within the area of welfare economics. This trend is being fuelled further by the empirical applications of the approach, which have provided evidence that it produces rather different results to the traditional income approach (e.g. Klasen, 2000, Alkire and Santos, 2010, Figari, 2012).

\section{Multidimensional Poverty Measurement}

As has already been pointed out, what is so fascinating about the capability approach is its approach to freedom of choice and individual responsibility. Poverty, according to Sen, is 'not a matter of low well-being, but of the inability to pursue well-being' (Sen, 1992, p. 110). In order to measure poverty as the inability to pursue well-being, a minimum capability set needs to be determined that comprises those basic or central capabilities that a person needs in order to be able to pursue his or her goals in life, i.e. his or her well-being. The definition acknowledges that the capability or opportunity of the poor to develop their human capital and reach their full potential is limited by external circumstances, such as socio-economic background, race, gender, religion, health, or nutrition.

Likewise, the issue is not inequality of well-being but inequality in the ability to pursue well-being. This definition already indicates Sen's concern to base considerations of distributive justice, i.e. the 'deep need for impartiality between individuals' (Sen 1992, p. 21), on the "right" kind of inequality (Sen, 1997, p. 12). This right kind of inequality is inequality among the poor, or inequality in the opportunity of individuals to pursue whatever they have reason to value. It wastes human capital, not as a result of individual choice, but due to being enforced on people by discrimination of any sort, e.g. by their socio-economic background, race, gender or religion, but also by adversity, such as poor health resulting from sicknesses 
or accidents etc. This is the kind of inequality we would want to avoid out of considerations of justice.

The kind of inequality we may not want to avoid is the inequality that results directly from freedom of choice. As an example, consider two individuals with the same capability set, i.e. with the same opportunity to pursue their goals in life. Whereas the first person chooses to enjoy life and work only if unavoidable, the other person makes great efforts to invest in his or her human capital in order to make his or her life count. While it is not in the interest of the capability approach to compare, let alone evaluate, the two different lifestyles, being as they are the result of individual choice, it nevertheless has to ask the question of how to deal with the results of the different choices. It is obvious that the second person will end up with a much larger capability set than the first person, i.e. there will be a high degree of inequality of capabilities.

However, this is a type of inequality that we may not want to fight. The reason is that there exists a tension between the objective of equal capabilities on one hand and the objective of overall expansion of capabilities, which I will refer to as "efficiency", on the other. ${ }^{5}$ Sen argues, '[a]n attempt to achieve equality of capabilities - without taking note of aggregative considerations - can lead to severe curtailment of the capabilities that people can altogether have' (Sen, 1992, pp. 7-8). Inequality in this case would reward the effort and investments made by the second person that are more than likely to lead to an overall expansion of capabilities from which society as a whole would benefit.

These considerations are strongly corroborated by the results of a recent study by Marrero and Rodriguéz (2010). The authors argue that the reason the relationship between inequality and growth is still controversial in academic literature is that this differentiation between inequality of opportunity and inequality of choice has not been made. Utilising a panel data set for 23 U.S. states, they demonstrate that no significant relationship can be established between overall inequality and growth whereas a robust and significantly positive relationship exists between growth and inequality of choice, and a robust and significantly negative relationship between growth and inequality of opportunity.

\footnotetext{
${ }^{5}$ Please note that there are two ways to apply the concept of efficiency. The first is a dynamic interpretation, i.e. it refers to the fact that rewarding effort and investments leads to higher growth and thus 'generally enhancing individual advantages, no matter how distributed' (Sen, 1992, p. 136). The second one is a static interpretation, i.e. describing a redistribution that improves the advantages of some individuals without worsening the situation of the others. It is the latter interpretation that is utilized in this thesis.
} 
These considerations forcefully demonstrate why inequality among the poor is crucial for any evaluation of poverty and explain Sen's strong opposition to poverty indices that neglect inequality (Sen, 1992, p. 103):

\begin{abstract}
'It can now be asked whether the two together would provide an adequate informational base for poverty measurement? The answer, briefly, is: no. $\mathrm{H}$ and I [i.e. poverty incidence and intensity] together still cannot be adequate, since neither pays any attention to the distribution of income among the poor. [...] Intensification of the more acute deprivation cannot be outweighed by the increase in the income of the person who was less poor even to start with.'
\end{abstract}

He further argues that by ignoring inequality among the poor, one might very well end up with anti-poverty policies (Sen 1992, p. 105).

But the considerations also explain his claim for a clear distinction between the concepts of distributive justice and efficiency that, though of course closely related, are obviously not the same. Acknowledging the tension between them is crucial. Though efficiency and distributive justice considerations make the same strong case against inequality of opportunity, they might very well differ in their ways of fighting it. In fact, one could easily consider changes in situations that are just, but not efficient and vice versa:

Just, but not efficient: Consider, for instance, a situation with two "comparable" individuals, i.e. individuals with the same individual characteristics facing the same circumstances. Now assume that one individual has access to seven right shoes and three left shoes, but the other individual has access to three right shoes and seven left shoes. The situation might easily be considered just, as the two otherwise "comparable" individuals have the same capability to wear three pairs of shoes. However, it is also deeply inefficient. By a simple exchange, both individuals could easily expand their capability sets from the opportunity to wear three pairs of shoes to the opportunity to wear five pairs of shoes.

Efficient, but not just: Consider the same initial situation of two "comparable" individuals with seven right and three left shoes and three right and seven left shoes, respectively. Now assume a simple exchange in the course of which individual one hands four of his/her seven shoes over to individual two. Individual one has still the same opportunity to wear three pair of shoes as before, while individual two has now the opportunity to wear seven pairs of shoes. This exchange is definitely efficient, it is, however, unjust - why should only the second individual gain from the exchange when both individuals could improve their situation?

And yet, current approaches to multidimensional poverty measurement that actually resulted out of Sen's capability approach do not account properly for his forceful claims. 
Well-known and widely-used multidimensional poverty indices either account for distributive justice or for efficiency, treating the two concepts as one and the same and thereby neglecting the tension that exists between them. It is the purpose of this volume to provide ways to overcome this methodological weakness in order to be able to evaluate the changes that a sound operationalisation of the capability approach will induce in poverty measurement. 


\section{Chapter 1}

\section{Efficiency and Distributive Justice in Ordinal Poverty Indices: The Correlation Sensitive Poverty Index}

The focus of this chapter is on the two concepts of distributive justice and efficiency within the context of ordinal multidimensional poverty measurement. Though a clear distinction exists between these two concepts they are usually equated. In fact, in the ordinal context inequality between dimensions is usually considered as the spread of simultaneous deprivations across the population. This narrow definition accounts for the concept of distributive justice but not for efficiency. This chapter suggests to define inequality between dimensions as the association-sensitive spread of simultaneous deprivations across the population. This more holistic approach captures both concepts of distributive justice and efficiency and facilitates the introduction of a new axiom as well as a new identification method which in turn provide the basis for a new class of multidimensional poverty indices. The latter is unique in the sense that it is the first ordinal class of additive poverty indices that is able to take care of considerations of distributive justice and efficiency at the same time. A representative of this class is the Correlation Sensitive Poverty Index (CSPI) that is exemplarily applied to the Indian DHS 2005 data set and illustrates the empirical relevance of the new methodological approach.

\section{$1.1 \quad$ Introduction}

The fact that poverty is a multidimensional phenomenon is undisputed, even in the income poverty literature. In fact, income is not supposed to be important per se but rather to serve as an indicator for economic resources that enable individuals to satisfy their multidimensional needs. In order to satisfy that purpose, two main assumptions have to be imposed: i) the existence of complete and perfect markets, and ii) perfect substitutability among all poverty dimensions. The appropriateness of these assumptions, however, has been increasingly 
questioned and finally led to a multidimensional measurement approach (e.g. Rawls, 1971; Sen, 1985; Drèze and Sen, 1989; UNDP, 1995).

Equally undisputed is the fact that inequality is detrimental to poverty reduction and human development in general (e.g. Fields, 1999; Kanbur and Lustig, 1999; Kanbur, 2000; Ravallion, 2001; White and Anderson, 2001; Bourguignon, 2003; Bourguignon, 2004; Ravallion, 2007; Grimm et al., 2010). It also nourishes hazardous social tensions and conflicts and even political violence (e.g. Muller and Seligson, 1987; Deininger, 2003). Thus, Sen (1976) requires any reasonable poverty index to be sensitive to inequality. In fact, if poverty is defined as the "lower end" of well-being, then every poverty index is a function of attribute distribution, a fact that shifts inequality into the centre of every poverty analysis.

In a multidimensional framework, poverty attributes are no longer restricted to a perfect substitute relationship. Inequality is no longer confined to the spread of dimension-specific achievements but in addition comprises the joint distribution of attributes across a population. The former has become known as intra-personal inequality (Kolm, 1977) and has been adequately captured by majorization properties ${ }^{6}$.

This chapter claims that inter-personal inequality (Atkinson and Bourguignon, 1982) - or inequality between dimensions -has not been adequately considered. In fact, in the context of ordinal poverty indices it is commonly equated with the distribution of simultaneous deprivations across the population (Chakravarty and D'Ambrosio 2006, Jayaraj and Subramanian 2010), an equation that fails to consider the relationship that exists between different poverty dimensions. However, the neglect of association-sensitivity leads to a neglect of the concept of efficiency that is not the same as distributive justice - though both concepts are closely related.

In response, this chapter introduces a new property that is based on a broader definition of inter-personal inequality as the association-sensitive spread of simultaneous deprivations across a population, and conditions the extent to which an "inequality increasing switch" increases poverty on the relationship among attributes. The new property is utilised to derive a new, uniquely characterised class of correlation sensitive poverty indices that is the first ordinal and additive measure able to account for considerations of distributive justice as well as efficiency. In order to demonstrate the empirical implications of the new approach, this chapter closes with a comparison of the poverty rates for 29 Indian states and union territories

\footnotetext{
${ }^{6}$ Majorization properties establish criteria to compare different possibilities to distribute wealth in a society. Given the two vectors of equal length $\mathbf{x}=\left(x_{1}, \ldots, x_{n}\right)$ and $\mathbf{x}^{\prime}=\left(x_{1}^{\prime}, \ldots, x_{n}^{\prime}\right)$ with $\sum_{i=1}^{n} x_{i}=\sum_{i=1}^{n} x_{i}^{\prime}$, a majorization property can be defined as $\mathbf{x} \geq_{M} \mathbf{x}^{\prime}: \Leftrightarrow \sum_{i=1}^{n-1} x_{(i: n)} \geq \sum_{i=1}^{n-1} x_{(i: n)}^{\prime}$ where $x_{(1: n)} \geq x_{(2: n)} \geq \ldots \geq x_{(n: n)}$.
} 
as calculated by of one of the most popular ordinal poverty indices, the Multidimensional Poverty Index (MPI) to those calculated by the new Correlation Sensitive Poverty Index (CSPI).

The chapter proceeds as follows. The second section provides a brief introduction in the theoretical background of the chapter. Section three lays the axiomatic foundation for the derivation and decomposition of the new class of indices that are derived in section four and utilised in the empirical application presented in section five. Section six concludes. Proofs are relegated to the appendix.

\subsection{Theoretical Background}

Composite poverty indices are derived in two main steps, i.e. the identification step to identify those who are poor, and the aggregation step to aggregate the individual poverty characteristics into the overall poverty index (Sen 1976). This section concentrates on the identification step. In a multidimensional framework, the identification of the poor requires two choices: $i$ ) the choice of poverty dimensions, indicators, thresholds and weights in order to identify those who are deprived, and ii) the choice of an appropriate method to identify the poor within the group of the deprived. Though the choices taken in the first step are crucial, they go well beyond the scope of this chapter whose theoretical approach can be applied to whatever poverty dimensions, indicators, weights and thresholds have been chosen in the first step. The following is a summary of the notations and definitions used throughout the chapter. Let $\mathbb{R}^{\mathrm{k}}$ denote the Euclidean $k$-space, and $\mathbb{R}_{+}^{\mathrm{k}} \subset \mathbb{R}^{\mathrm{k}}$ the non-negative $k$-space. Further, let $\mathbb{N}$ denote the set of positive integers. $\mathbf{N}=\{1, \ldots, n\} \subset \mathbb{N}$ represents the set of $n$ individuals and $\mathbf{D}=\{2, \ldots, d\} \subset \mathbb{N}$ the set of $d$ poverty dimensions captured by a set of poverty attributes $\mathbf{K}=\{2, \ldots, k\} \subset \mathbb{N}$. Let $\mathbf{a} \in \mathbb{R}_{+}^{\mathbf{K}}$ denote the weight vector for the different attributes with $a_{j}>0$ for all $j=1, \ldots, k$ and $\sum_{j=1}^{k} a_{j}=1$.

I refer to the quantity of an attribute with which an individual is endowed as an achievement. The achievement vector of individual $i$ is represented by $\mathbf{x}_{i \cdot}=\left(x_{i 1}, \ldots, x_{i k}\right)$. The achievement matrix of a society with $n$ individuals is represented by $\mathbf{X} \in \mathbb{R}_{+}^{\mathrm{NK}}$ where the $i j$ th entry represents the achievement $x_{i j}$ of individual $i$ in attribute $j$. Let $X_{\mathrm{n}}$ be the set of possible achievement matrices of population size $n$ and $X=\mathrm{U}_{\mathbb{N}} \subset_{N} X_{n}$ the set of all possible achievement matrices. I further refer to the poverty threshold of attribute $j$ as $z_{j}$. Thus, individual $i$ is deprived in attribute $j$ whenever her achievement falls short of the respective threshold, i.e. 
whenever $x_{i j}<z_{j} . \mathbf{z} \in \mathbb{R}_{++}^{\mathbf{K}}$ represents the vector of the chosen poverty thresholds and $\mathbf{Z}$ the set of all possible vectors of poverty thresholds.

I define a poverty index as a function $P: X \times \mathbf{Z} \rightarrow \mathbb{R}$. For any poverty threshold vector $\mathbf{z} \in \mathbf{Z}$, society $\mathcal{A}$ has a higher poverty level than society $\mathcal{B}$ if and only if $P\left(\mathbf{X}^{\mathcal{A}} ; \mathbf{z}\right) \geq P\left(\mathbf{X}^{\mathfrak{B}} ; \mathbf{z}\right)$ for any $\mathbf{X}^{\Re}, \mathbf{X}^{\mathfrak{B}} \in X$.

The main methods for the identification of the poor are the aggregate poverty line approach and the component poverty approach. Under the aggregate poverty line approach, the identification of the poor is based upon a function $\varphi: \mathbb{R}^{\mathrm{k}} \times \mathbb{R}^{\mathrm{k}} \rightarrow \mathbb{R}$ that aggregates individual achievements in all poverty dimensions. A person is poor if and only if his/her aggregation function is negative, i.e. $\varphi\left(\mathbf{x}_{i} ; \mathbf{z}\right)<0$. The unique feature of this approach is that it allows compensation between attributes below and above threshold levels among those who are poor (Weak Focus Axiom).

This limits the application of this approach to a cardinal context and does therefore not allow an ordinal-cardinal comparison as aspired in this volume.

Therefore, the following analysis is based upon the second method, the component poverty approach that builds upon an attribute-wise evaluation of poverty. All attributes are considered to be essential in the sense that a failure to achieve the threshold level automatically implies deprivation no matter what the achievements are in other dimensions, i.e. compensation is restricted to attributes below threshold levels (Strong Focus Axiom). In order to describe the component poverty approach, let $\mathbf{c}_{i}=\left(c_{i 1}, \ldots, c_{i k}\right)$ represent the deprivation vector of individual $i$ such that $c_{i j}=1$ if $x_{i j}<z_{j}$ and $c_{i j}=0$ if $x_{i j} \geq z_{j}$. Further, let $\delta_{i}=\sum_{j \in\{1, \ldots, k\} c_{i j}=1} a_{j}$ denote the sum of weighted deprivations suffered by individual $i$ and let $S_{j}(\mathbf{X})$ - or simply $S_{j}$ - denote the set of individuals who are deprived with respect to attribute $j$. The identification of the poor is based on a function $\rho: \mathbb{R}_{+}^{\mathbf{K}} \times \mathbb{R}_{++}^{\mathbf{K}} \rightarrow\{0,1\}$, i.e. an identification function such that individual $i$ is poor if $\rho\left(\mathbf{c}_{i} ; \mathbf{z}\right)=1$ and not poor if $\rho\left(\mathbf{c}_{i \cdot} ; \mathbf{z}\right)=0$.

Three specifications of the identification function have been suggested so far. The union method is based on the (rather strong) assumption that all attributes are perfect complements ${ }^{7}$.

\footnotetext{
${ }^{7}$ I follow the Auspitz-Lieben-Edgeworth-Pareto (ALEP) definition of substitutability and complementarity. The ALEP definition considers two attributes to be substitutes if their second cross partial derivatives are positive. Intuitively, an increase in one attribute decreases poverty the less the higher the achievements in the second attribute. In the same way, attributes are considered to be complements, when the respective cross partial derivatives are negative and independent in case they are zero.
} 
Thus, deprivation in one attribute equals deprivation in all attributes so that each person who is deprived is considered to be poor: $\rho_{U}\left(\mathbf{c}_{i \cdot} ; \mathbf{z}\right)=\left\{\begin{array}{lll}1 & \text { if } & \max \left\{\mathbf{c}_{i \cdot}\right\}=1 \\ 0 & \text { if } & \max \left\{\mathbf{c}_{i \cdot}\right\}=0\end{array}\right.$.

In other words, already the loss in one attribute cannot be compensated, thus, there is no need to consider inequality.

The intersection method, on the other hand, is based on the (equally strong) assumption that all attributes to be perfect substitutes. Thus, poverty occurs solely in case of deprivation in all attributes so that only those individuals are considered poor who are deprived in every single attribute: $\rho_{I S}\left(\mathbf{c}_{i} ; \mathbf{z}\right)=\left\{\begin{array}{lll}1 & \text { if } & \min \left\{\mathbf{c}_{i .}\right\}=1 \\ 0 & \text { if } & \min \left\{\mathbf{c}_{i}\right\}=0\end{array}\right.$.

In other words, the loss in any attribute can always be completely compensated through other attributes - unless a person is deprived in all attributes. Therefore, only those persons are considered poor, who suffer the highest degree of deprivation. This resembles in a way a Rawlsian "maxi-max" principle (Rawls, 1971) which requires the maximum improvement of the situation of the worst off, i.e. those with the maximum number of deprivations (Jayaraj and Subramanian, 2010, p. 56).

Both approaches are obviously extreme cases, relying on extreme assumptions, and consequently yielding poverty rates that are plainly inapplicable, being either far too high or far too low (Bérenger and Bresson, 2010; Alkire and Foster, 2011).

Mack and Lindsay (1985) were the first to formulate the idea for an intermediate method as a kind of a loophole that was explicitly introduced as the dual cut-off method by Foster (2007) and Alkire and Foster (2007, 2011). The intermediate method defines a minimum level of weighted deprivation so that individual $i$ is considered poor if his/ her sum of weighted deprivations is at least $\delta_{I M}^{\min }: \rho_{I M}\left(\mathbf{c}_{i \cdot} ; \mathbf{z}\right)=\left\{\begin{array}{lll}1 & \text { if } & \delta_{i} \geq \delta_{I M}^{\min } \\ 0 & \text { if } & \delta_{i}<\delta_{I M}^{\min }\end{array}\right.$.

Please note that the intermediate method comprises union and intersection method as extreme cases, i.e. in case $\delta_{I M}^{\min } \hat{=} \max \left\{\mathbf{c}_{i .}\right\}=1$ and $\delta_{I M}^{\min } \hat{=} \min \left\{\mathbf{c}_{i .}\right\}=1$, respectively.

Though the intermediate method is a convenient way out of the dilemma of extreme poverty rates, its theoretical justification is questionable. Apart from the fact that the choice of $\delta_{I M}^{\min }$ is arbitrary, the whole method is based on the indirect assumption that up to $\delta_{I M}^{\min }$ attributes are perfect substitutes whereas the same attributes are considered perfect complements from $\delta_{I M}^{\min }$ onwards. 
Figure 1.01 Existing Identification Methods
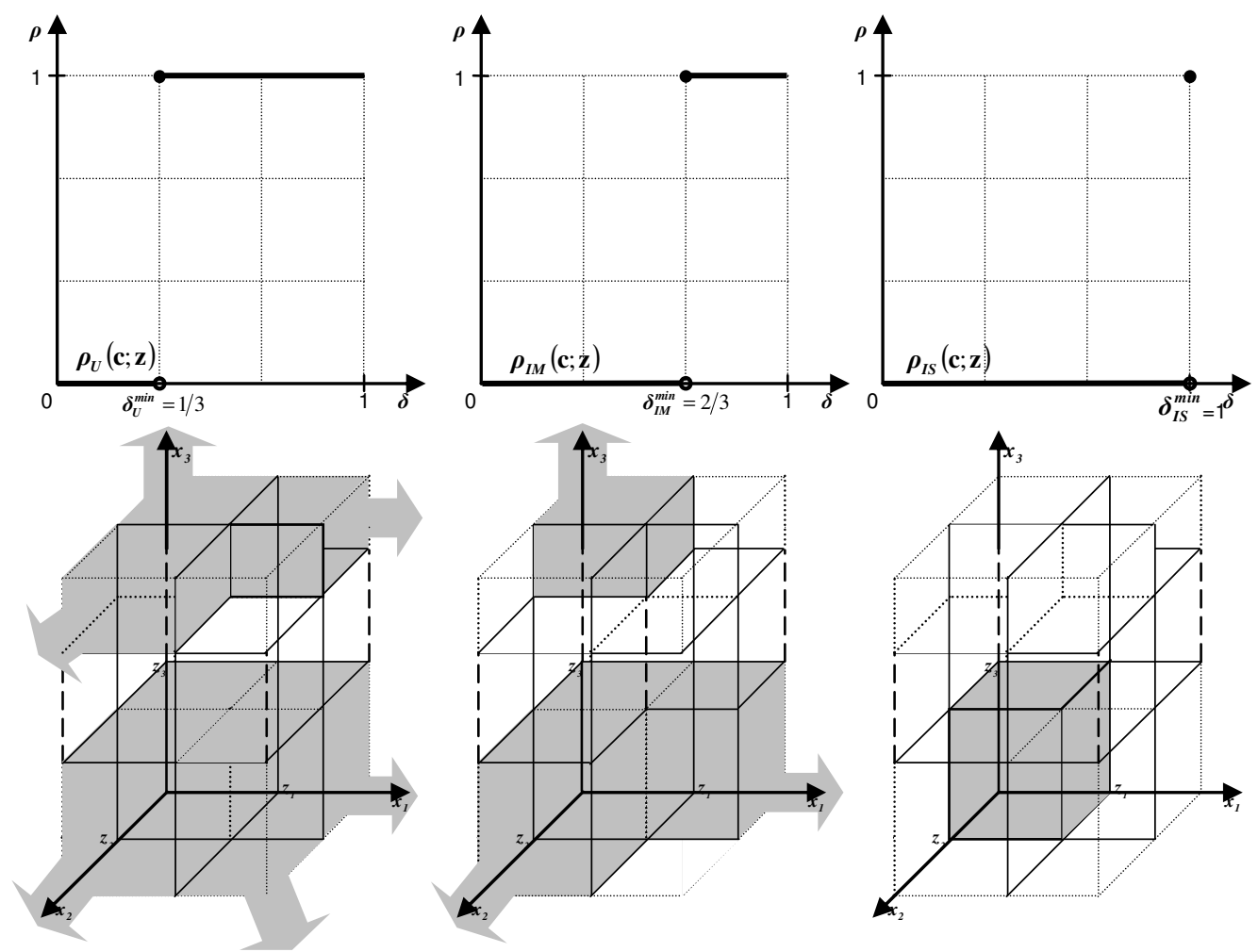

Figure 1 provides a graphical illustration of the different identification methods for the case of three equally weighted attributes. The upper part of the figure shows the different identification functions. The horizontal axis indicates the sum of weighted deprivations from which an individual suffers and the vertical axis whether the respective individual is poor $(\rho=1)$ or not $(\rho=0)$. The identification function of the union method, $\rho_{U}(\mathbf{c} ; \mathbf{z})$, equals one for any individual that is deprived, i.e. for any $i$ with $\delta_{i} \geq 1 / 3=\delta_{U}^{\min }$. The intermediate method is illustrated for a cut-off level of two third, i.e. $\rho_{I M}(\mathbf{c} ; \mathbf{z})=1$ for any $i$ with $\delta_{i} \geq 2 / 3=\delta_{I M}^{\min }$. The identification function of the intersection method, $\rho_{I S}(\mathbf{c} ; \mathbf{z})$, equals one only in case an individual is deprived in all attributes, i.e. for any $i$ with $\delta_{i}=1=\delta_{I S}^{\min }$.

The bottom part of the figure shows the attribute constellations according to which individuals are classified as poor. The three axes indicate the quantities for the three attributes $\left(x_{1}, x_{2}, x_{3}\right)$ and the respective threshold levels $\left(z_{1}, z_{2}, z_{3}\right)$. Shaded areas highlight those attribute constellations that indicate poverty. Obviously, the union method relates almost all, the intersection method only one attribute constellation to poverty. The intermediate approach is the only method that yields "reasonable" poverty rates - however based on a questionable assumption concerning the relationship between attributes.

Summarizing, all three methods rely on extreme assumptions regarding the relationship between attributes which are either all perfect substitutes or all perfect complements. These 
assumptions do not only exclude every other form of relationship between attributes, they also imply that inequality in the distribution of attributes between individuals is either irrelevant (in the case of perfect complements) or of utmost importance (in the case of perfect substitutes).

This chapter demonstrates that once the two concepts of distributive justice and efficiency are properly taken care of, they imply a new identification method which provides a theoretically-guided way out of the dilemma of inapplicable poverty rates. The argument is related to one made by Dasgupta and Ray in 1986 who observed that inequality issues can either be included in poverty reduction policies by formulating a respective axiom in the aggregation step or by choosing the "right" poverty line, i.e. by tailoring the poverty line to the size of the budget such that all who are poor according to this line are lifted out of poverty. They point out that though the two procedures are equivalent in an 'arithmetical sense', the former seems to be the more appropriate one to employ.

\subsection{The Aggregation Step}

Current research concentrates on four main approaches to aggregate individual poverty characteristics into one composite index: i) the fuzzy set approach, ii) the distance function approach, iii) the information theory approach, and iv) the axiomatic approach. In this volume I will concentrate on the axiomatic approach as it best fits the purpose of my research. Thus, I will briefly introduce the first three approaches before turning to a more detailed discussion of the axiomatic approach. ${ }^{8}$

\subsubsection{The Fuzzy Set Approach}

The theoretical foundation of the fuzzy set approach was developed by Zadeh (1965) and is based on the consideration that it may sometimes be impossible to precisely define certain sets of objects. Whereas it is always possible to decide for some objects whether they belong to a certain set or not there may very well remain objects for which such a precise statement cannot be made. As a consequence, these objects are considered to form a kind of fuzzy subset.

Let there be any set $T$ and let $t$ be any element of this set. $A$ is characterised as a fuzzy set of $T$ by a so called membership function $\omega_{A}(t)$ that assigns to each element in $T$ a number in

\footnotetext{
${ }^{8}$ For a detailed discussion of the different aggregation methods see Deutsch and Silber, 2005; Kakwani and Silber, 2008; Maasoumi and Lugo, 2008.
} 
the interval [0,1]. If $\omega_{A}(\bar{t})=0$ for any $\bar{t} \in T, \bar{t}$ does not belong to $A$. If $\omega_{A}(\hat{t})=1$ for any $\hat{t} \in T, \hat{t}$ belongs completely to $A$. However, in case $0<\omega_{A}(\tilde{t})<1$ for any $\tilde{t} \in T$, element $\tilde{t}$ belongs only partially to $A$, with the degree of membership the greater the higher the value of $\omega_{A}(\tilde{t})$. $^{9}$

Applied in the context of multidimensional poverty, it can be said that some individuals lack so many resources that they certainly should be classified as poor whereas the welfare level of others is as high that they should certainly not be classified as poor at all. However, there will be individuals for whom such a classification is not that obvious. In fact, there will be many individuals who are deprived in some dimensions and not deprived in others. To those, the fuzzy set approach would attribute different degrees of membership to the set of the poor.

The use of fuzzy measures in the context of poverty measurement allows for an imprecise borderline between the poor and the non-poor which seems to be a promising way to capture the vagueness inherent in the concept of poverty. Yet, the very advantage of this approach is also its main disadvantage when it comes to the practical application: the impreciseness of the approach renders poverty comparisons extremely difficult. This is the main reason why I decided not to use the fuzzy set approach in the following analysis.

\subsubsection{The Distance Function Approach}

The distance function approach is a concept widely used in efficiency analysis and especially in production economics as a mean to summarize the range of information resulting from the multi-output nature of production into merely one dimension. Distance functions can be differentiated according to their input or output orientation. Intuitively, the output distance function $D_{\text {out }}(x, y)$ measures the extent to which an output vector $y \in R_{++}^{M}$ can be increased given a fixed input vector $x \in R_{++}^{N}$. Let $P(x)$ be defined as the output set of all output vectors $y$ which can be produced by the input vector $x$. The production possibility frontier $\operatorname{PPF}(x)$ depicts the maximum among the output combinations that can be produced by input vector $x$.

The distance function for a specific output combination measures the distance between this combination and $P P F(x)$ as the inverse of the factor by which the production could be

\footnotetext{
${ }^{9}$ Note that fuzzy poverty measures are not measures of the intensity of poverty, but instead address the vagueness of poverty (Qizilbash and Clark 2005).
} 
increased for a given input vector $x$, i.e. $D_{\text {out }}(x, y)=\min \{\theta:(y / \theta) \in P(x)\}$ where $\theta$ is a scalar that measures the distance to $\operatorname{PPF}(x) .{ }^{10}$

Similarly, the input distance function $D_{\text {in }}(x, y)$ measures the extent to which an input vector $x \in R_{++}^{N}$ can be reduced given a fixed output vector $y \in R_{++}^{M}$. Let $L(y)$ be defined as the input set of all input vectors $x$ which can produce the output vector $y$. The isoquant $I Q(y)$ depicts the minimum among the input combinations that can produce a certain output vector $y$.

The distance function for a specific input combination measures the distance between this combination and $I Q(y)$ as the inverse of the factor by which the input quantities could be reduced for a given output vector $y$, i.e. $D_{i n}(x, y)=\max \{\rho:(x / \rho) \in L(y)\}$ where $\rho$ is a scalar that measures the distance to $\operatorname{IQ}(y) .{ }^{11}$

Summarizing, the distance function approach aggregates the information that results from a multidimensional phenomenon into one single dimension - a clear methodological similarity to multidimensional poverty measurement. In addition, the approach does not rely on any kind of assumption concerning prices or behaviour, a property that makes a transfer to the thematic field of multidimensional poverty measurement appear particularly desirable.

Lovell et al. (1994) were the first to apply the distance function approach to the thematic context of well-being. They interpret the input vector $x$ as a vector of resources and the output vector $y$ as a vector of functionings. Then they derive a multidimensional index of well-being by first utilizing the concept of the input distance function to aggregate individual achievements which are in a second step transformed into functionings via an output distance function. In other words, what the distance function approach in effect does is to aggregate individual achievements in the various poverty dimensions into a single index with individual $i$ being poor whenever his/her aggregate index falls below some chosen poverty line.

Thus, this approach aggregates poverty dimensions first and determines one poverty line for the aggregate vector afterwards (instead of poverty thresholds for each dimension). It resembles therefore the traditional one-dimensional poverty measures (e.g. the income-based approach with a broader concept of income) and therefore does, strictly speaking, not count as

\footnotetext{
${ }^{10}$ It can be shown that $D_{\text {out }}(x, y)$ is non-decreasing, positively linearly homogenous and concave in $y$ and decreasing in $x$. Additionally, $D_{\text {out }}(x, y) \leq 1$ if $y$ belongs to $P(x)$ and $D_{\text {out }}(x, y)=1$ if $y$ lies on $P P F(x)($ Coelli et al., 1998).

${ }^{11}$ It can be shown that $D_{i n}(x, y)$ is non-decreasing, positively linearly homogenous and concave in $x$ and decreasing in $y$. Additionally, $D_{i n}(x, y) \leq 1$ if $x$ belongs to $L(y)$ and $D_{i n}(x, y)=1$ if $x$ lies on $I Q(y)$ (Coelli et al., 1998).
} 
a truly multidimensional approach. This is the main reason why this approach is not followed up in the following analysis.

\subsubsection{The Information Theory Approach}

Cover and Thomas (2006, p. 1) provide the following description of the information theory approach: 'Information Theory answers two fundamental questions in communication theory: What is the ultimate data compression (answer: the entropy $H$ ), and what is the ultimate transmission rate of communication (answer: the channel capacity $C$ ).'

There exists a specific result concerning the entropy which is extremely valuable in the context of inequality and poverty measurement: two entropies are equal if, and only if, underlying distributions are identical. As a result, there exists a basic measure for the divergence between two distributions, namely the difference between their entropies, the socalled relative entropy (Maasoumi and Lugo 2008, p. 8).

Due to this appealing property, the concept of entropy has been applied to the analysis of multidimensional inequality (Maasoumi, 1986) and later to the analysis of multidimensional poverty. The basic idea is that since all poverty indices are functions of the distribution of identified attributes and the distribution of attributes contains all the information about the attributes, a poverty index should be required to have a distribution as 'close' as possible to the multivariate distribution of attributes. In other words, the respective relative entropy is to be minimized.

Let $S_{i}$ denote the aggregator function for individual $i$ which depends on his/her achievement vector $\mathbf{x}_{i}=\left(x_{i 1}, \ldots, x_{i k}\right)$. Then, in order to derive an 'optimal' poverty index, Maasoumi and Lugo $(2008$, p. 8) consider the following weighted average of the relative entropy divergences between the aggregator functions $\left(S_{1}, S_{2}, \ldots, S_{n}\right)$ and each vector of attributes $\mathbf{x}_{j .}=\left(x_{1 j}, x_{2 j} \ldots, x_{n j}\right)$ that is to be minimized:

$$
D_{\lambda}(S, X ; w)=\sum_{j=1}^{k} w_{j}\left[\sum_{i=1}^{n} S_{i}\left[\frac{\left(S_{i} / x_{i j}\right)^{-\lambda}-1}{\lambda(\lambda-1)}\right]\right]
$$

with $w_{j}$ being the weight attached to the Generalized Entropy divergence from attribute $j$. Minimizing $D_{\lambda}(S, X ; w)$ with respect to $S_{i}$ leads to the following two 'optimal' Information Theory aggregation functions (Maasoumi and Lugo, 2008, p. 8):
(1) $S_{i} \propto\left[\sum_{j=1}^{k} w_{j} x_{i j}^{\lambda}\right]^{1 / \lambda}$ if $\lambda \neq 0$
(2) $S_{i} \propto \prod_{j=1}^{k}\left(x_{i j}\right)^{w_{j}}$ if $\lambda=0$ 
Maasoumi and Lugo (2008) claim that the information theory approach represents the most elegant way to aggregate attributes as it summarizes the information inherent in all attributes in an efficient manner. However, as has been discussed before, efficiency should not be the only criterion for the aggregation of attributes. Rather, any aggregation should be based on the two concepts of efficiency and distributive justice. This is the main reason why the following analysis is not based on the information theory approach. However, the latter has the valuable advantage that it can easily be utilized as an add-on in almost every poverty measurement approach. ${ }^{12}$ In other words, any class of poverty measures can be scrutinized as to whether it is efficient in the sense of the information theory approach or not, something which will be made use of in the following analysis.

\subsubsection{The Axiomatic Approach}

Chakravarty, Mukherjee and Ranade (1998) were the first to apply the axiomatic approach in order to derive multidimensional poverty indices. The idea behind this approach is to define a list of desirable properties that a poverty index should satisfy. This list is then utilized to derive those classes of poverty indices that satisfy these properties. Thus, the axiomatic approach provides the most transparent way to take care of value judgments by explicitly defining properties that poverty indices may or may not satisfy. It is this specific property that makes the axiomatic approach so appealing for the analysis of the interplay between the two concepts of efficiency and distributive justice, the main objective of this volume.

Most of the axioms that are introduced in the following analysis are so-called core axioms that have been derived by the generalization and extension of the core axioms of the one-dimensional framework to fit the multidimensional framework (e.g. Chakravarty, Mukherjee and Ranade 1998, Bourguignon and Chakravarty 1999, Tsui 2002, Bourguignon and Chakravarty 2003, Chakravarty and Silber 2008). However, it is the main message of this volume that the axioms that have been derived so far are unable to sufficiently capture the interplay between the two concepts of efficiency and distributive justice. In fact, as will be demonstrated in the following, they either account for the one or the other but not for both.

This chapter concentrates on the axiomatic foundation for ordinal poverty measures; the second chapter will do the same for the cardinal case.

\footnotetext{
${ }^{12}$ Note, however, that the information theory approach reaches far beyond being a simple add-on for other methods. Indeed, it opens the way to more generalized poverty measures which are more sophisticated than, for instance, the average functions of the axiomatic approach (Maasoumi and Lugo 2008, p. 8).
} 


\section{The Axiomatic Foundation for Ordinal Poverty Measures}

\section{Non-Distributional Axioms}

Anonymity (AN): For any $\mathbf{z} \in Z$ and $\mathbf{X} \in X_{\mathrm{n}}, P(\mathbf{X} ; \mathbf{z})=P(\boldsymbol{\Pi X} ; \mathbf{z})$ where $\boldsymbol{\Pi}$ is any permutation matrix of appropriate order.

AN states that any characteristic of persons apart from the attributes $j$ are irrelevant for poverty measurement.

Monotonicity (MN): For any $\mathbf{z} \in Z$ and $\mathbf{X}, \mathbf{X}^{\prime} \in X_{\mathrm{n}}$, if for any individual $h$ and any attribute $l$ $x_{h l}=x_{h l}^{\prime}+\beta, \quad$ such $\quad$ that $\quad x_{h l}^{\prime}<z_{l}, \beta>0$, and $\quad x_{i l}=x_{i l}^{\prime} \forall i \neq h, \quad x_{i j}=x_{i j}^{\prime} \forall j \neq l, \forall i$, then $P\left(\mathbf{X}^{\prime} ; \mathbf{z}\right) \leq P(\mathbf{X} ; \mathbf{z})$.

MN requires poverty measures not to increase if, ceteris paribus, the condition of individual $h$ that is poor with respect to attribute $l$ improves.

Principle of Population (PP): If for any $\mathbf{z} \in Z, \mathbf{X} \in X_{\mathrm{n}}$, and $m \in \mathbb{N} \mathbf{X}^{m}$ is a $m$-fold replication of $\mathbf{X}$, then $P\left(\mathbf{X}^{m} ; \mathbf{z}\right)=P(\mathbf{X} ; \mathbf{z})$.

PP ensures that poverty measures do not depend on population size, thereby allowing crosspopulation and cross-time comparisons.

Strong Focus (SF): For any $\mathbf{z} \in Z$ and $\mathbf{X} \in X_{\mathrm{n}}$, if for any individual $h$ and any attribute $l$ $x_{h l} \geq z_{l}, x_{h l}^{\prime}=x_{h l}+\beta, \beta>0$, and $x_{i l}^{\prime}=x_{i l} \forall i \neq h, x_{i j}^{\prime}=x_{i j} \forall j \neq l, \forall i$, then $P(\mathbf{X} ; \mathbf{z})=P\left(\mathbf{X}^{\prime} ; \mathbf{z}\right)$.

SF demands that giving a person more of an attribute with respect to which this person is not deprived will not change the poverty measure - even if the person is deprived in (an)other attribute(s). SF provides the theoretical foundation for the component poverty approach ${ }^{13}$.

Subgroup Decomposability (SD): For any $\mathbf{X}^{1}, \ldots, \mathbf{X}^{v} \in X_{\mathrm{n}}$ and $\mathbf{z} \in Z$, $P\left(\mathbf{X}^{1}, \mathbf{X}^{2}, \ldots, \mathbf{X}^{v} ; \mathbf{z}\right)=\sum_{l=1}^{v} n_{l} / n P\left(\mathbf{X}^{l} ; \mathbf{z}\right) \quad$ with $n_{l}$ being the population size of $\operatorname{subgroup} \mathbf{X}^{l}, l=1, \ldots, v$ and $\sum_{l=1}^{v} n_{l}=n$.

$\mathrm{SD}$ requires overall poverty to be expressible as the population share weighted average of subgroup poverty levels. It therefore allows the decomposition of overall poverty into the poverty levels of population subgroups according to ethnic, spatial or other criteria which makes it a valuable property for policy makers.

Factor Decomposability (FD): For any $\mathbf{z} \in Z$ and $\mathbf{X} \in X_{\mathrm{n}}, P(\mathbf{X} ; \mathbf{z})=\sum_{j=1}^{k} a_{j} P\left(x_{. j} ; z_{j}\right)$

FD facilitates the decomposition of poverty measures into different attribute combinations. Joint fulfilment of FD and SD allows a twofold decomposition of overall poverty according to

\footnotetext{
${ }^{13}$ There exists a weaker version of this property that provides the theoretical foundation for the aggregate poverty line approach (e.g. Maasoumi and Lugo 2008).
} 
subgroup-attribute combinations that improves the targeting of poverty-alleviating policies. A rather restricting implication of FD is that it requires poverty indices to be additive.

Normalization (NM): For any $\mathbf{z} \in Z$ and $\mathbf{X} \in X_{\mathrm{n}}, P(\mathbf{X} ; \mathbf{z})=1$ if $x_{i j}=0 \forall i, j$ and $P(\mathbf{X} ; \mathbf{z})=0$ if $x_{i j} \geq z_{j} \forall i, j$. Thus, $P(\mathbf{X} ; \mathbf{z}) \in[0,1]$.

$\mathrm{NM}$ is a technical property that simply requires poverty measures to be equal to zero in case all individuals are non-poor and equal to one in case all individuals are completely deprived.

\section{Distributional Axioms}

I will now turn to the axioms that deal specifically with inter-personal inequality. In the context of ordinal poverty measures, inter-personal inequality is captured by a majorization criterion proposed by Chakravarty and D'Ambrosio (2006) in the context of social exclusion and formally introduced in the context of multidimensional poverty measurement by Jayaraj and Subramanian (2010):

Equality-Promoting Change (EPC): For any $\mathbf{z} \in Z$ and $\mathbf{X}, \mathbf{X}^{\prime} \in X_{\mathrm{n}}, \mathbf{X}^{\prime}$ is obtained from $\mathbf{X}$ by an equality-promoting change, if for some individuals $g$ and $h$, $\mathbf{c}_{g}^{\prime}=\mathbf{c}_{g}+1, \mathbf{c}_{h}^{\prime}=\mathbf{c}_{h}-1, \mathbf{c}_{g}^{\prime} \leq \mathbf{c}_{h}^{\prime}$ and $\mathbf{c}_{i}^{\prime}=\mathbf{c}_{i} \forall i \neq g, h$.

Nonincreasingness under Equality-Promoting Change (NEPC): For any $z \in Z$ and $\mathbf{X}, \mathbf{X}^{\prime} \in \quad X_{\mathrm{n}}$ if $\mathbf{X}^{\prime}$ is obtained from $\mathbf{X}$ by an equality-promoting change, then $P(\mathbf{X} ; \mathbf{z}) \geq P\left(\mathbf{X}^{\prime} ; \mathbf{z}\right)$.

A change is equality-promoting whenever the difference in the number of simultaneously suffered deprivations between two individuals is reduced. Jayaraj and Subramanian (2010) claim that such an equality-promoting change should not increase poverty.

However, the equality-promoting change does not distinguish between the attributes that are affected by the change, i.e. is insensitive to any kind of association among attributes and is therefore unable to account for the concept of efficiency. However, '[a]n attempt to achieve equality of capabilities - without taking note of aggregative considerations - can lead to severe curtailment of the capabilities that people can altogether have' (Sen, 1992, pp. 7-8).

In fact, an increase in inter-personal inequality can increase or decrease poverty, depending on the assumed relationship among attributes. More precisely, poverty will increase as long as there is no complementary relationship among attributes since the two concepts of distributive justice and efficiency work in the same direction. However, this is no longer true in the case of complements. In this case, distributive justice considerations suggest an increase in poverty while efficiency considerations suggest a decrease in poverty. It 
depends on the importance that is attributed to distributive justice considerations as well as on the degree of complementarity which of the effects will predominate in the end.

A rather vivid example is provided by Duclos, Sahn and Younger (2006, p. 950) who observe that complementarities exist between the two poverty dimensions education and nutrition as better nourished children learn better. If the degree of this complementarity is strong enough, the authors argue, it might even overcome the 'usual ethical judgement' (Duclos, Sahn and Younger, 2006, p. 950) that favours those deprived in more dimensions so that overall poverty would actually decrease in case education would be transferred from the poorly to the better nourished.

In order to capture this idea, I introduce the concept of an Inequality Increasing Switch:

Inequality Increasing Switch (IIS): Define $d_{i}=\#\left\{c_{i j} \mid c_{i j}=1\right\}$. Then, for two individuals $g$ and $h$ such that $d_{g}>d_{h}>1$, Matrix $\mathbf{X}$ is said to be obtained from matrix $\mathbf{X}^{\prime}$ by an inequality increasing switch of attribute $l \quad$ if $\quad x_{h l}^{\prime}<z_{l}<x_{g l}^{\prime}, \quad x_{g l}=x_{h l}^{\prime}, x_{h l}=x_{g l}^{\prime} \quad$ and $x_{i l}=x_{i l}^{\prime} \forall i \neq g, h ; x_{i j}=x_{i j}^{\prime} \forall j \neq l, \forall i$.

An inequality increasing switch is a switch of attributes that increases (reduces) the number of deprivations suffered by the person with higher (lower) initial deprivation.

Based on the Inequality Increasing Switch, I introduce the following property that captures the interplay between the two concepts of distributive justice and efficiency:

Sensitivity to Inequality Increasing Switch (SIIS): For any $\mathbf{z} \in Z$ and $\mathbf{X}, \mathbf{X}^{\prime}, \mathbf{X}^{\prime \prime} \in X_{\mathrm{n}}$ if $\mathbf{X}^{\prime}$ is obtained from $\mathbf{X}$ by an inequality increasing switch of non-complementary attributes, then $P(\mathbf{X} ; \mathbf{z}) \leq P\left(\mathbf{X}^{\prime} ; \mathbf{z}\right)$. Further, if $\mathbf{X}^{\prime \prime}$ is obtained from $\mathbf{X}$ by an inequality increasing switch of complement attributes, then $P(\mathbf{X} ; \mathbf{z}) \underset{\leq}{\geq}\left(\mathbf{X}^{\prime \prime} ; \mathbf{z}\right) \leq P\left(\mathbf{X}^{\prime} ; \mathbf{z}\right)$.

As mentioned before, in the case of a non-complementary relationship between attributes there is no conflict between the concepts of distributive justice and efficiency. Thus, SIIS is equivalent to NEPC, i.e. an inequality increasing switch should not decrease. The situation changes, however, in the case of complements. In this case, the concepts of distributive justice and efficiency work in different directions and it depends on the importance attributed to distributive justice considerations as well as on the degree of complementarity whether poverty increases or decreases through an inequality increasing switch.

In the following section I will utilise the axioms developed here in order to derive a new class of poverty indices and compare it to the main other indices that have been developed in an ordinal context. 


\subsection{Correlation-Sensitive Poverty Indices}

There exist different ways to ensure that a poverty index satisfies the new property SIIS. There exists, however, only one way to ensure that a poverty index satisfies FD and SIIS at the same time. This way is especially interesting as it is usually assumed that any poverty index that complies with FD is unavoidably insensitive to inter-personal inequality. The following theorem demonstrates that in case of a modification in the identification step there exists a class of poverty measures that is able to comply with both properties, FD and SIIS.

Theorem 1. A multidimensional poverty measure $\mathrm{P}$ satisfies AN, NM, MN, SF, PP, FD, SD and SIIS if and only if there exists $\mathbf{a}=\left(a_{1}, \ldots, a_{k}\right) \in \mathbb{R}_{++}^{\mathbf{K}}$ with $\sum_{j=1}^{k} a_{j}=1$ such that for all $n \in \mathbf{N}$ and $\mathbf{X} \in X_{\mathrm{n}}: P(\mathbf{X} ; \mathbf{z})=1 / n \sum_{i \in S_{j}} f\left(\mathbf{c}_{i \cdot}\right) \sum_{j \in\{1, \ldots, k\}, c_{i j}=1} a_{j}$

where $f\left(\mathbf{c}_{i .}\right): \quad \mathbb{R}_{+}^{\mathbf{K}} \times \mathbb{R}_{++}^{\mathbf{K}} \rightarrow(0,1]$ is non-decreasing in $d_{i}=\#\left\{c_{i j} \mid c_{i j}=1\right\}$ and has a nondecreasing (nonincreasing) marginal ${ }^{14}$ in case attributes are considered to be substitutes (complements).

The new family of poverty indices is based on a multiple step identification function that replaces the single step function currently used for the identification of the poor:

$$
\rho_{C S}\left(\mathbf{c}_{i} ; \mathbf{z}\right)=\left\{\begin{array}{cll}
f\left(\mathbf{c}_{i .}\right) & \text { if } & \max \left\{c_{i .}=1\right\} \\
0 & \text { if } & \max \left\{c_{i .}=0\right\}
\end{array}\right.
$$

Instead of dividing a society into the poor and non-poor, the new function differentiates between the non-poor on one hand and different degrees of poverty severity on the other (Rippin 2009). While the new function is always nondecreasing in the number of deprivations, the marginal increase in poverty severity is lower the higher the substitutability between attributes. Consequently, individuals suffer different degrees of poverty severity dependent on i) the attributes in which they are simultaneously deprived, and ii) the kind of relationship that exists among these attributes.

For the purpose of illustration, consider the following identification function:

$$
\rho_{c s}\left(\mathbf{c}_{i} ; \mathbf{z}\right)=\left\{\begin{array}{lll}
\delta_{i}^{\alpha} & \text { if } & \max \left\{\mathbf{c}_{i \cdot}\right\}=1 \\
0 & \text { if } & \max \left\{\mathbf{c}_{i .}\right\}=0
\end{array}\right.
$$

Equivalent to the case of intra-personal inequality, alpha is interpreted as an indicator for inequality aversion. However, different from the case of intra-personal inequality, the choice of alpha directly implies assumptions about the relationship among attributes and vice versa.

\footnotetext{
${ }^{14}$ A function $f(x)$ has a nondecreasing marginal if $f\left(x_{g}+1\right)-f\left(x_{g}\right) \geq f\left(x_{h}+1\right)-f\left(x_{h}\right)$ whenever $x_{g} \geq x_{h}$.
} 
More precise, higher inequality aversion implies the assumption of a higher level of substitutability among attributes and vice versa. Figure 1.02 illustrates this fact without loss of generality for the case of three equally weighted attributes.

Figure 1.02 The Correlation Sensitive Identification Method
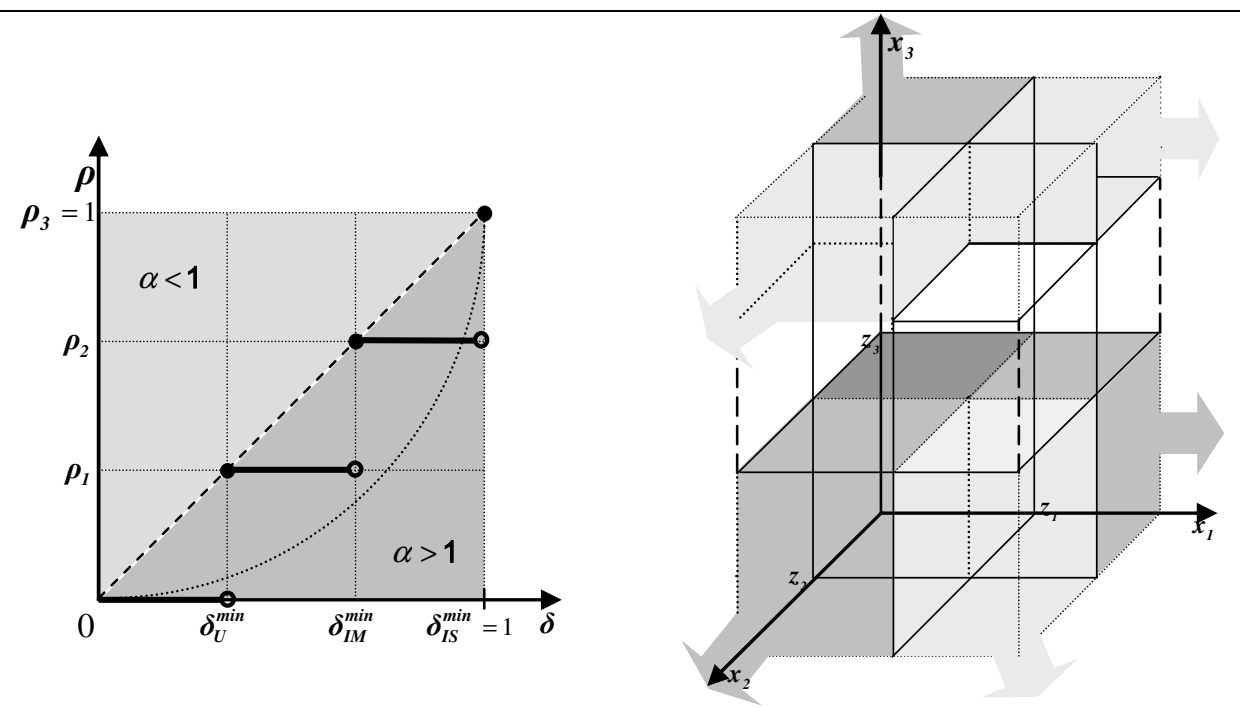

The lighter shade indicates the area in which $\alpha<1 . \rho_{c s}\left(\mathbf{c}_{i} ; \mathbf{z}\right)$ approximates a concave shape, i.e. the increase in the level of poverty severity is marginally decreasing in the number of deprivations as the loss in even one attribute can hardly be compensated. This, in turn, implies a low level of inequality aversion: as already the loss in one attribute can barely be compensated, there is in fact no need for a strong focus on inequality. In the extreme case of $\alpha=0$, inequality can be neglected as attributes are perfect complements and therefore nonsubstitutable. This is the representation of the union method.

The darker shade highlights the area in which $\alpha>1 . \rho_{c s}\left(\mathbf{c}_{i} ; \mathbf{z}\right)$ approximates a convex shape, i.e. the increase in the level of poverty severity is marginally increasing in the number of deprivations: the lack in only one attribute leads to a rather low poverty degree as $k-1$ other attributes can compensate for the loss. Obviously, there is a need for a strong focus on inequality as expressed in a high value of alpha. In the extreme case of perfect substitutes and infinite inequality aversion, i.e. $\alpha \rightarrow \infty$, the resulting identification function approximates a sort of "Rawlsian" function where poverty is defined solely by those who suffer maximal deprivation (see Jayaraj and Subramanian 2010). This is the representation of the intersection method.

Finally, in the case $\alpha=1, \rho_{c s}\left(\mathbf{c}_{i} ; \mathbf{z}\right)$ coincides with the $45^{\circ}$ degree line. Attributes are independent, implying that inequality aversion is linearly increasing in the number of deprivations. Please note that the new identification method does not naturally comprise the 
intermediate method - though it can easily be modified to do so ${ }^{15}$. It's a direct consequence of the method's inconsistent assumptions about the relationship among attributes.

The second part of figure 1.02 illustrates the different attribute constellations according to which individuals are characterised as poor. To account for all those who are deprived in at least one dimension acknowledges the essentiality of every single poverty attribute and, as we will see later, ensures the efficiency of the new class of poverty indices. This number, however, is too high to be useful; in fact, it coincides with the poor as identified by the union method. Therefore, the distinction between different degrees of poverty severity as illustrated in the figure by different shades is crucial. One possibility to use the additional information for poverty headcount purposes is to divide the poor in different groups. For example, in the empirical application in section five of this chapter, I differentiate between the deprivation affected $\left(0<\delta_{i} \leq 1 / 3\right)$, the poor $\left(1 / 3<\delta_{i} \leq 2 / 3\right)$ and the severely poor $\left(2 / 3<\delta_{i} \leq 1\right)$.

In the following, I will compare this new class of poverty indices with three other wellknown classes of ordinal poverty measures. Thereby, I will make also use of the valuable property of the information theory approach, i.e. that it can be utilized as an add-on tool to scrutinize whether a particular poverty index makes efficient use of the information that is available. As described in section 1.3.3, the 'optimal' Information Technology (IT) aggregation function in the case of ordinal poverty measures is (Maasoumi and Lugo, 2008):

$$
S_{i} \propto\left[\sum_{j \in\{1, \ldots, k\}: c_{j j}=1} w_{j}\right]^{1 / \lambda}
$$

with $\lambda \neq 0$, and $w_{j}$ being the weight attached to the Generalized Entropy divergence from each attribute. This is the formula I will utilize in the following comparison.

The multidimensional Bossert, Chakravarty and D'Ambrosio class of poverty measures

$$
P_{B C D}=\left[1 / n \sum_{i \in S_{j}}\left[\sum_{j \in\{1, \ldots, k\}\}_{c i j}=1} a_{j}\right]^{r}\right]^{1 / r}
$$

with $r \geq 1$.

The restriction $r \geq 1$ excludes the possibility that attributes might be complements, i.e. the index satisfies NEPC but not SIIS. In other words, it is only able to capture considerations of distributive justice but neglects the concept of efficiency. This becomes also obvious if one

15 Define $\hat{\rho}_{C S}\left(\mathbf{c}_{i} ; \mathbf{z}\right)=\left\{\begin{array}{ccc}\delta_{i}^{\alpha} & \text { if } & \delta_{i} \geq \delta_{I M}^{\min } \\ 0 & & \text { otherwise }\end{array}\right.$. For the special case $\alpha=0, \hat{\rho}_{c s}\left(\mathbf{c}_{i} ; \mathbf{z}\right)$ coincides with the intermediate method itself. 
compares $P_{B C D}$ to formula (1.06): this class of poverty measures satisfies the efficiency criterion of the information theory approach only if a non-complementary relationship exists between attributes, i.e. only in case the two concepts of distributive justice and efficiency do not diverge.

\section{The multidimensional Chakravarty and D'Ambrosio class of poverty measures}

$$
P_{C D}=1 / n \sum_{i \in S_{j}}\left[\sum_{j \in\{1, \ldots, k\}, c_{i j}=1} a_{j}\right]^{r}
$$

$P_{C D}$ imposes the same non-decreasing marginal assumption as $P_{B C D}$, i.e. $r \geq 1$, rejecting the possibility that attributes might be complements. Thus, like $P_{B C D}$, this class of poverty indices accounts only for considerations of distributive justice but not efficiency, a fact that is concealed as long as the two concepts do not diverge, i.e. in case a non-complementary relationship exists between attributes.

The multidimensional Alkire and Foster class of poverty measures

$$
M_{0}=1 / n \sum_{\substack{\left.i \in S_{j} \\ j \in\{1, \ldots, k\}\right\}_{c}=1 \\ \wedge \delta_{i} \geq \delta_{I M}^{\text {min }}}} a_{j}
$$

Contrary to the other two classes of poverty measures, aggregation of attributes according to $M_{0}$ is never efficient in an information theory sense: the optimal IT aggregation function imposes a union definition of poverty (Maasoumi and Lugo 2008, p. 10). This comes at no surprise as any other definition of poverty - including the intermediate method - discards information about the distribution of attributes that would be required to ensure efficiency. Thus, this class of poverty measures does not fulfil NEPC nor the more demanding SIIS, i.e. it is insensitive to both, considerations of distributive justice as well as efficiency.

The multidimensional Correlation-Sensitive class of poverty measures

$$
P_{C S}(\mathbf{X} ; \mathbf{z})=1 / n \sum_{i \in S_{j}} \delta_{i}^{\alpha} \sum_{j \in\{1, \ldots, k\} c_{i j}=1} a_{j}=1 / n \sum_{i \in S_{j}} \delta_{i}^{\alpha+1}
$$

Contrary to $M_{0}, P_{C S}$ aggregates attributes in an efficient manner. Its specific identification function includes all deprivation affected individuals and therefore all available information on the distribution of attributes of those who are deprived. In this sense it is comparable to the union method that is mandatory for IT efficiency. At the same time, the fact that $\alpha$ can assume positive as well as negative values ensures that attributes can be substitutes or complements (or independent). Therefore, it is the only class of poverty indices that accounts for both concepts, distributive justice as well as efficiency. Also note that the 
final formula of $P_{C S}\left(1 / n \sum_{i \in S_{j}} \delta_{i}^{\alpha+1}\right)$ is the result of a two-step calculation with $\sum_{i \in S_{j}} \delta_{i}^{\alpha}$ being the individual weight calculated in the identification step. This clear separation between the identification and the aggregation step enables the additivity of the index in the aggregation step - and thus the fulfillment of FD.

Table 1.01 compares the different classes of poverty measures according to the properties that they do or do not satisfy.

Table 1.01 Axiomatic Foundation of Selected Ordinal Poverty Measures

\begin{tabular}{lccccc}
\hline Axioms & & $M_{0}$ & $P_{C D}$ & $P_{B C D}$ & $P_{C S}$ \\
\hline Anonymity (AN) & $\checkmark$ & $\checkmark$ & & $\checkmark$ & $\checkmark$ \\
Monotonicity (MN) & & $\checkmark$ & $\checkmark$ & $\checkmark$ & $\checkmark$ \\
Principle of Population (PP) & & $\checkmark$ & $\checkmark$ & $\checkmark$ \\
Strong Focus (SF) & & $\checkmark$ & $\checkmark$ & $\checkmark$ & $\checkmark$ \\
Normalization (NM) & $\checkmark$ & $\checkmark$ & $\checkmark$ & $\checkmark$ \\
Subgroup Decomposability (SD) & & $\checkmark$ & $\checkmark$ & $\times$ & $\checkmark$ \\
Factor Decomposability (FD) & & $\times$ & $\times$ & $\checkmark$ \\
Sensitivity to Inequality Increasing Switch (SIIS) & $\times$ & $(\checkmark)$ & $(\checkmark)$ & $\checkmark$ \\
\hline
\end{tabular}

Please note that the special merit of $P_{C S}$ is the simultaneous fulfilment of SIIS, FD and SD. Though $P_{C S}$ is additive, the precondition for the fulfilment of FD, the differentiation between degrees of poverty severity ensures its compliance with considerations of distributive justice as well as efficiency.

The close connection between the classes of poverty indices presented above can easily be established for the case of equal weights, i.e. $a_{j}=1 / k$ for all $j \in\{1, \ldots, k\}^{16}$, and under the condition $\alpha>0$, i.e. in case attributes are substitutes.

\section{Proposition 1.}

Define $\pi_{\alpha}=\sum_{j=1}^{k}(j / k)^{\alpha} H_{j}$ where $H_{j}=q_{j} / n$ is the proportion of the population that is deprived in $j$ poverty dimensions and $\alpha>0$. Then:

$$
\begin{gathered}
P_{C D}=\sum_{j=1}^{k}(j / k)^{\alpha+1} H_{j}=\pi_{\alpha+1} \\
M_{0}=\sum_{j=1}^{k}(j / k)^{1} H_{j}=\pi_{1} \\
P_{B C D}=\left(\sum_{j=1}^{k}(j / k)^{\alpha+1} H_{j}\right)^{1 /(\alpha+1)}=\left(\pi_{\alpha}\right)^{1 /(\alpha+1)} \\
P_{C S}=\sum_{j=1}^{k}(j / k)^{\alpha+1} H_{j}=\pi_{\alpha+1}
\end{gathered}
$$

Please note that $P_{C D}$ and $P_{C S}$ come to the same result $\pi_{\alpha+1}$. However, the former reaches it by assuming a substitute relationship among attributes. The latter, on the other hand, reaches it through the additional weight that individuals receive in the identification step in dependence of their degree of poverty severity. It is this differentiation between the

\footnotetext{
${ }^{16}$ Compare Jayaraj and Subramanian (2010), p.55
} 
identification and the aggregation step that ensures the additivity of $P_{C S}$. Thus, $M_{0}$ and $P_{C S}$ are the only additive classes of poverty measures and the remainder of the chapter focuses on a comparison of the two.

\section{Poverty Decompositions}

A direct consequence of the insensitivity of $M_{0}$ to inter-personal inequality is that the index can only be decomposed into the product of poverty incidence and intensity (Alkire and Santos 2010).

\section{Proposition 2.}

$$
M_{0}=\hat{H} \cdot \hat{\mu}(\boldsymbol{\delta})
$$

with the censored headcount ratio $\hat{H}=(\hat{q} / n)$ measuring the incidence of poverty, and

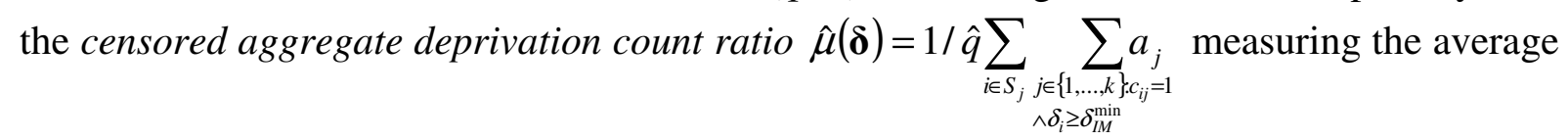
intensity of poverty breadth.

$P_{C S}$, on the other hand, can be decomposed into all three 'I's of poverty' (Jenkins and Lambert 1997): incidence, intensity and inequality. Let me first introduce an inequality index that is based on the distribution of deprivation counts and is a representative of the Generalized Entropy (GE) class:

$$
G E(\mathbf{x})= \begin{cases}{\left[1 / n\left(\theta^{2}-\theta\right)\right] \sum_{i=1}^{n}\left[\left(x_{i} / \mu\right)^{\theta}\right]-1} & \theta \neq 0,1 \\ -1 / n \sum_{i=1}^{n} \log \left(x_{i} / \mu\right) & \theta=0 \\ 1 / n \sum_{i=1}^{n}\left(x_{i} / \mu\right) \log \left(x_{i} / \mu\right) & \theta=1\end{cases}
$$

With the GE class for $\theta \neq 0,1, P_{C S}$ can be decomposed as follows.

\section{Proposition 3.}

$$
P_{C S}(\mathbf{X} ; \mathbf{z})=H \cdot \hat{\mu}(\boldsymbol{\delta})^{\theta} \cdot\left[1+2 G E_{\theta}(\boldsymbol{\delta})\right]
$$

with $\theta=\alpha+1$, the headcount ratio $H=(q / n)$ measuring the incidence of poverty, the aggregate deprivation count ratio $\hat{\mu}(\boldsymbol{\delta})=(1 / q) \sum_{i \in S_{j}} \sum_{j \in\{1, \ldots, k\} c_{i j}=1} a_{j}$ measuring the intensity of poverty breadth and the GE inequality measure of deprivation counts $G E(\boldsymbol{\delta})=\left[1 / q\left(\theta^{2}-\theta\right)\right] \sum_{i \in S_{j}}\left[\delta_{i} / \mu(\boldsymbol{\delta})\right]^{\theta}-1$ capturing inter-personal inequality.

Please note that the poverty incidence is in fact the headcount of the deprivation affected, a number too high to be useful as has already been pointed out. But again, the new class of 
poverty indices has a special feature that is illustrated in the empirical application: it allows the decomposition of poverty incidence according to different levels of poverty severity.

The theoretical analysis revealed that the new class of correlation-sensitive poverty indices has a couple of methodological advantages over other ordinal poverty measures. In order to analyse the empirical implications of the methodological advantages, the remainder of the chapter concentrates on an application to poverty in India.

\subsection{Poverty in India}

In this section I will illustrate the implications of the theoretical modification developed in this chapter with the Indian data set from the Demographic and Health Surveys (DHS), nationally representative surveys that are mainly funded by the US Agency for International Development (USAID). In particular, I will compare the MPI as the prominent representative of $M_{0}$ with a representative of $P_{C S}$ called Correlation Sensitive Poverty Index (CSPI):

$$
C S P I=1 / n \sum_{i \in S_{j}} \delta_{i} \sum_{j \in\{1, \ldots, k\}: c_{i j}=1} a_{j}=1 / n \sum_{i \in S_{j}} \delta_{i}^{2}
$$

In words, the CSPI is based on the assumption of independent attributes $(\alpha=1)$. The empirical application follows the MPI's choices with regard to poverty dimensions, indicators, thresholds and weights. Though these choices, especially with regard to equal weighting, have been the subject of severe criticism, their utilisation in this context is for illustrative matters only and occurs without loss of generality: the new measures can as well be applied to other choices of parameters, including weights.

Thus, in accordance with the MPI, I focus on the three equally weighted poverty dimensions education, health and living standards which are captured by an overall of ten indicators. The health dimension is measured by the two equally weighted indicators nutrition and child mortality. A household is deprived in nutrition if any woman in reproductive age (15-49) has a Body Mass Index (BMI) smaller than 18.5 or if any child has a weight-for-age z-score below -2.0 according to World Health Organization (WHO) statistics. A household is deprived in child mortality if any child regardless of age died in the household.

The education dimension is captured by the two equally weighted indicators years of schooling and child enrolment. A household is deprived in years of schooling if no member of the household has at least five years of schooling. It is deprived in child enrolment if any child in school age is not attending school. 
The living standard dimension is measured by an overall of six equally weighted indicators, cooking fuel, sanitation, water, electricity, floor and assets. With regard to the first five indicators, a household is deprived if Millennium Development Goals (MDGs) standards are not met. A household is asset deprived if it neither owns a car or truck nor possesses more than one of the following small assets: tv, radio, telephone, refrigerator, bicycle or motorbike.

Figure 1.03 provides an overview of the different dimensions, indicators and thresholds used for the calculation of the MPI and CSPI:

Figure 1.03 The Structure of the MPI

\begin{tabular}{|c|c|c|c|}
\hline Dimension & Main Capability & Indicator & Threshold (Household Level) \\
\hline \multirow[t]{2}{*}{ Health } & \multirow[t]{2}{*}{ Bodily Health } & Nutrition & $\begin{array}{l}\text { At least one of the following: } \\
\text { 1. At least one woman age } 15-49 \text { with BMI }<18.5 \\
\text { 2. At least one child with weight-for-age } \mathrm{z} \text {-score }<-2.0\end{array}$ \\
\hline & & $\begin{array}{l}\text { Child Mortality } \\
\text { Rate }\end{array}$ & At least one child under the age of 18 died \\
\hline \multirow{2}{*}{ Education } & \multirow{2}{*}{$\begin{array}{l}\text { Senses, } \\
\text { Imagination and } \\
\text { Thought }\end{array}$} & Schooling & No member with at least five years of schooling \\
\hline & & Enrolment & At least one child in school age not enrolled \\
\hline \multirow{6}{*}{$\begin{array}{l}\text { Living } \\
\text { Standards }\end{array}$} & \multirow{6}{*}{$\begin{array}{l}\text { Bodily Health } \\
\text { Control over } \\
\text { Environment }\end{array}$} & Cooking Fuel & Harmful material is used for cooking (straw, dung, coal etc.) \\
\hline & & Sanitation & Toilet either unhygienic (no facility, open lid, etc.) or shared \\
\hline & & Water & Water source is unprotected or more than 30 minutes away \\
\hline & & Electricity & No access to electricity \\
\hline & & Floor & Floor material is earth, sand or dung \\
\hline & & Assets & Not more than one small asset and no car/truck \\
\hline
\end{tabular}

For a first illustration of the difference between the MPI and the CSPI consider the following example that is taken from the 2005 Indian DHS (yes indicates deprivation).

Table 1.02 A Comparison of Five Indian Households (DHS 2005)

\begin{tabular}{|c|c|c|c|c|c|c|c|c|c|c|c|c|}
\hline \multirow{2}{*}{ HH } & \multicolumn{2}{|c|}{ Education } & \multicolumn{2}{|c|}{ Health } & \multicolumn{4}{|c|}{ Living Standards } & \multirow{2}{*}{ MPI } & \multirow{2}{*}{ CSPI } \\
\cline { 2 - 10 } & Years & Attendance & Mortality & Nutrition & Electricity & Water & Sanitation & Flooring & Cooking & Assets & & \\
\hline 1 & yes & yes & yes & no & yes & yes & no & yes & no & yes & 0.722 & 0.522 \\
\hline 2 & yes & no & no & no & yes & yes & no & yes & yes & no & 0.389 & 0.151 \\
\hline 3 & no & no & no & no & yes & yes & yes & yes & yes & no & 0.000 & 0.077 \\
\hline 4 & no & yes & no & no & no & no & yes & no & no & no & 0.000 & 0.049 \\
\hline 5 & no & yes & no & no & no & no & no & no & no & no & 0.000 & 0.028 \\
\hline
\end{tabular}

The five households are deprived in different indicators, with household 1 suffering from the highest and household 5 from the lowest number of simultaneous deprivations. The most obvious difference between the two poverty indices is the fact that the MPI considers only households 1 and 2 as poor whereas the CSPI acknowledges the fact that poverty exists in all five households - though to very different degrees.

Consider, for instance, household 2 and 3. Both households are deprived in five indicators yet with different weights. Household 2 is deprived in years of schooling (weight: 
1/6), electricity, water, floor and cooking fuel (weight: 1/18). Therefore, its MPI value is $1 \times 1 / 6+4 \times 1 / 18=0.389$. Household 3 on the other hand is deprived in electricity, water, sanitation, floor and cooking fuel (weight: 1/18). Therefore, its MPI value is zero as $5 \times 1 / 18=0.278<1 / 3$. Thus, due to the difference in the weights, household 3 is not considered poor whereas household 2 enters the index with the considerable weight of 0.389 . The CSPI, on the other hand considers both households as poor though household 2 enters the index with a weight of 0.151 and household 3 with a weight of 0.077 - which seems to reflect the situation of the two households more appropriately.

The following table provides an overview of poverty rates and decompositions for 29 Indian states and union territories according to MPI and CSPI.

Table 1.03 Poverty Rates and Decompositions Selected Indian States

\begin{tabular}{|c|c|c|c|c|c|c|c|}
\hline \multirow[t]{2}{*}{ State } & \multicolumn{3}{|c|}{ MPI } & \multicolumn{4}{|c|}{ CSPI } \\
\hline & Value & $\hat{H}$ & $\hat{\mu}(\boldsymbol{\delta})$ & Value & $H$ & $\mu(\boldsymbol{\delta})$ & $G E(\boldsymbol{\delta})$ \\
\hline Kerala & 0.051 & 0.126 & 0.405 & 0.041 & 0.852 & 0.182 & 0.226 \\
\hline Delhi & 0.057 & 0.132 & 0.432 & 0.039 & 0.596 & 0.212 & 0.228 \\
\hline Goa & 0.084 & 0.195 & 0.429 & 0.054 & 0.705 & 0.234 & 0.195 \\
\hline Mizoram & 0.090 & 0.200 & 0.447 & 0.059 & 0.732 & 0.236 & 0.221 \\
\hline Punjab & 0.114 & 0.248 & 0.459 & 0.074 & 0.817 & 0.250 & 0.225 \\
\hline Himachal Pradesh & 0.121 & 0.294 & 0.412 & 0.074 & 0.895 & 0.253 & 0.148 \\
\hline Tamil Nadu & 0.132 & 0.311 & 0.425 & 0.077 & 0.847 & 0.261 & 0.170 \\
\hline Sikkim & 0.156 & 0.333 & 0.468 & 0.095 & 0.861 & 0.280 & 0.208 \\
\hline Maharashtra & 0.182 & 0.382 & 0.476 & 0.110 & 0.861 & 0.306 & 0.184 \\
\hline Uttaranchal & 0.185 & 0.398 & 0.464 & 0.109 & 0.833 & 0.317 & 0.148 \\
\hline Manipur & 0.185 & 0.398 & 0.465 & 0.112 & 0.951 & 0.295 & 0.178 \\
\hline Haryana & 0.188 & 0.399 & 0.473 & 0.114 & 0.883 & 0.309 & 0.180 \\
\hline Jammu and Kashmir & 0.193 & 0.411 & 0.470 & 0.116 & 0.889 & 0.313 & 0.166 \\
\hline Gujarat & 0.203 & 0.416 & 0.488 & 0.123 & 0.831 & 0.332 & 0.169 \\
\hline Andhra Pradesh & 0.206 & 0.439 & 0.468 & 0.120 & 0.881 & 0.321 & 0.161 \\
\hline Karnataka & 0.208 & 0.439 & 0.474 & 0.123 & 0.888 & 0.323 & 0.165 \\
\hline Nagaland & 0.247 & 0.487 & 0.508 & 0.154 & 0.949 & 0.348 & 0.171 \\
\hline Tripura & 0.266 & 0.534 & 0.497 & 0.160 & 0.953 & 0.360 & 0.149 \\
\hline Arunachal Pradesh & 0.277 & 0.536 & 0.517 & 0.171 & 0.914 & 0.375 & 0.166 \\
\hline Meghalaya & 0.300 & 0.554 & 0.541 & 0.190 & 0.920 & 0.395 & 0.162 \\
\hline West Bengal & 0.308 & 0.575 & 0.536 & 0.192 & 0.922 & 0.400 & 0.151 \\
\hline Assam & 0.318 & 0.603 & 0.527 & 0.196 & 0.937 & 0.407 & 0.129 \\
\hline Orissa & 0.339 & 0.630 & 0.538 & 0.213 & 0.950 & 0.420 & 0.134 \\
\hline Rajasthan & 0.342 & 0.631 & 0.542 & 0.216 & 0.939 & 0.426 & 0.132 \\
\hline Uttar Pradesh & 0.370 & 0.681 & 0.544 & 0.230 & 0.946 & 0.446 & 0.109 \\
\hline Chhattisgarh & 0.372 & 0.698 & 0.533 & 0.227 & 0.951 & 0.443 & 0.111 \\
\hline Madhya Pradesh & 0.374 & 0.681 & 0.550 & 0.236 & 0.940 & 0.452 & 0.113 \\
\hline Jharkhand & 0.443 & 0.748 & 0.593 & 0.296 & 0.960 & 0.506 & 0.103 \\
\hline Bihar & 0.480 & 0.795 & 0.604 & 0.323 & 0.972 & 0.529 & 0.093 \\
\hline
\end{tabular}

The results presented in table 1.03 reveal that, due to the additional cut-off, poverty according to the MPI is deflated in less poor and inflated in poorer states. At first glance this might seem as an advantage as it draws the attention to the poorest states, but these results are actually quite pernicious from a policy perspective. Due to the MPI's insensitivity with regard 
to inequality, highest attention is paid to those closest to the cut-off, and least attention to the poorest of the poor. Yet, in the poorest countries with the most severe budget constraints, targeting the neediest is of utmost importance. Again due to the additional cut-off, the MPI values for poverty incidence are deflated at the cost of an inflation of poverty intensity. In less poor states, poverty intensity is a multiple of poverty incidence which makes the values difficult to interpret. It is a great advantage of the CSPI that it avoids such distortions and allows for better targeting due to its sensitivity to inequality.

As already pointed out, CSPI poverty incidence is the headcount of the deprivation affected. The decision to consider all deprivation affected households is based on the assumption of the essentiality of each poverty attribute and ensures the IT efficiency of the index; the resulting headcount, however, is too high to be useful. But the new identification method additionally provides information about the different degrees of poverty severity that can be utilised to divide poor households in different groups. Here, I differentiate between the deprivation affected $\left(0<\delta_{i} \leq 1 / 3\right)$, the poor $\left(1 / 3<\delta_{i} \leq 2 / 3\right)$ and the severely poor $\left(2 / 3<\delta_{i} \leq 1\right)$.

Table 1.04 The CSPI and Selected Decompositions for India (DHS 2005)

\begin{tabular}{|c|c|c|c|c|c|c|c|c|c|c|c|c|c|c|c|c|}
\hline \multirow[t]{2}{*}{ State } & \multirow[b]{2}{*}{$\vec{v}$} & \multirow[b]{2}{*}{ 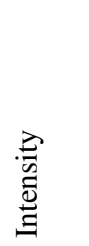 } & \multirow[b]{2}{*}{$\begin{array}{l}\stackrel{\gtrsim}{\Xi} \\
\stackrel{\Xi}{\Xi} \\
\stackrel{\Xi}{\Xi}\end{array}$} & \multicolumn{3}{|c|}{ Headcount } & \multicolumn{10}{|c|}{ Contribution of Indicators to Poverty } \\
\hline & & & & 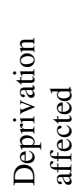 & ¿̈ & 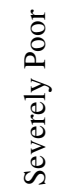 & 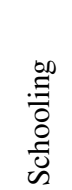 & 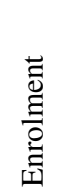 & 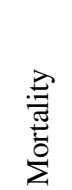 & : & 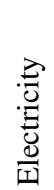 & 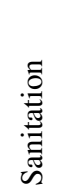 & $\begin{array}{l}\overline{\bar{J}} \\
\bar{E} \\
\bar{z}\end{array}$ & $\frac{\overline{8}}{I}$ & $\begin{array}{l}\bar{\Phi} \\
\text { II } \\
00 \\
\Xi 0 \\
0 \\
0 \\
0\end{array}$ & 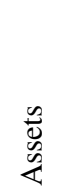 \\
\hline Andhra Pradesh & 0.120 & 0.321 & 0.161 & $44 \%$ & $39 \%$ & $5 \%$ & $14 \%$ & $9 \%$ & $13 \%$ & $23 \%$ & $2 \%$ & $11 \%$ & $2 \%$ & $5 \%$ & $11 \%$ & $9 \%$ \\
\hline Arunachal Pradesh & 0.171 & 0.375 & 0.166 & $38 \%$ & $41 \%$ & $13 \%$ & $14 \%$ & $19 \%$ & $13 \%$ & $17 \%$ & $4 \%$ & $8 \%$ & $3 \%$ & $4 \%$ & $10 \%$ & $9 \%$ \\
\hline Assam & 0.196 & 0.407 & 0.129 & $33 \%$ & $48 \%$ & $13 \%$ & $11 \%$ & $9 \%$ & $11 \%$ & $20 \%$ & $9 \%$ & $9 \%$ & $4 \%$ & $10 \%$ & $10 \%$ & $7 \%$ \\
\hline Bihar & 0.323 & 0.529 & 0.093 & $18 \%$ & $52 \%$ & $28 \%$ & $13 \%$ & $16 \%$ & $13 \%$ & $20 \%$ & $7 \%$ & $8 \%$ & $1 \%$ & $8 \%$ & $9 \%$ & $6 \%$ \\
\hline Chhattisgarh & 0.227 & 0.443 & 0.111 & $25 \%$ & $54 \%$ & $15 \%$ & $10 \%$ & $11 \%$ & $14 \%$ & $22 \%$ & $4 \%$ & $10 \%$ & $3 \%$ & $9 \%$ & $10 \%$ & $7 \%$ \\
\hline Delhi & 0.039 & 0.212 & 0.228 & $46 \%$ & $12 \%$ & $1 \%$ & $10 \%$ & $18 \%$ & $18 \%$ & $25 \%$ & $0 \%$ & $12 \%$ & $4 \%$ & $1 \%$ & $5 \%$ & $7 \%$ \\
\hline Goa & 0.054 & 0.234 & 0.195 & $51 \%$ & $18 \%$ & $1 \%$ & $7 \%$ & $9 \%$ & $8 \%$ & $32 \%$ & $1 \%$ & $11 \%$ & $6 \%$ & $7 \%$ & $11 \%$ & $7 \%$ \\
\hline Gujarat & 0.123 & 0.332 & 0.169 & $41 \%$ & $36 \%$ & $6 \%$ & $10 \%$ & $10 \%$ & $14 \%$ & $27 \%$ & $3 \%$ & $10 \%$ & $3 \%$ & $6 \%$ & $10 \%$ & $8 \%$ \\
\hline Haryana & 0.114 & 0.309 & 0.180 & $48 \%$ & $34 \%$ & $5 \%$ & $7 \%$ & $14 \%$ & $13 \%$ & $26 \%$ & $2 \%$ & $10 \%$ & $3 \%$ & $7 \%$ & $12 \%$ & $7 \%$ \\
\hline Himachal Pradesh & 0.074 & 0.253 & 0.148 & $60 \%$ & $29 \%$ & $1 \%$ & $4 \%$ & $4 \%$ & $11 \%$ & $33 \%$ & $1 \%$ & $14 \%$ & $4 \%$ & $7 \%$ & $15 \%$ & $9 \%$ \\
\hline Jammu \&Kashmir & 0.116 & 0.313 & 0.166 & $48 \%$ & $36 \%$ & $5 \%$ & $7 \%$ & $14 \%$ & $13 \%$ & $22 \%$ & $2 \%$ & $12 \%$ & $5 \%$ & $8 \%$ & $11 \%$ & $7 \%$ \\
\hline Jharkhand & 0.296 & 0.506 & 0.103 & $21 \%$ & $47 \%$ & $28 \%$ & $10 \%$ & $14 \%$ & $12 \%$ & $20 \%$ & $7 \%$ & $9 \%$ & $5 \%$ & $8 \%$ & $9 \%$ & $7 \%$ \\
\hline Karnataka & 0.123 & 0.323 & 0.165 & $45 \%$ & $38 \%$ & $5 \%$ & $9 \%$ & $12 \%$ & $13 \%$ & $25 \%$ & $2 \%$ & $11 \%$ & $3 \%$ & $5 \%$ & $11 \%$ & $8 \%$ \\
\hline Kerala & 0.041 & 0.182 & 0.226 & $73 \%$ & $12 \%$ & $0 \%$ & $3 \%$ & $7 \%$ & $9 \%$ & $33 \%$ & $4 \%$ & $3 \%$ & $9 \%$ & $3 \%$ & $19 \%$ & $10 \%$ \\
\hline Madhya Pradesh & 0.236 & 0.452 & 0.113 & $26 \%$ & $49 \%$ & $19 \%$ & $10 \%$ & $12 \%$ & $14 \%$ & $22 \%$ & $4 \%$ & $9 \%$ & $4 \%$ & $8 \%$ & $9 \%$ & $7 \%$ \\
\hline Maharashtra & 0.110 & 0.306 & 0.184 & $48 \%$ & $34 \%$ & $4 \%$ & $7 \%$ & $10 \%$ & $12 \%$ & $26 \%$ & $4 \%$ & $11 \%$ & $2 \%$ & $8 \%$ & $10 \%$ & $8 \%$ \\
\hline Manipur & 0.112 & 0.295 & 0.178 & $55 \%$ & $35 \%$ & $4 \%$ & $6 \%$ & $13 \%$ & $11 \%$ & $18 \%$ & $3 \%$ & $10 \%$ & $8 \%$ & $12 \%$ & $11 \%$ & $7 \%$ \\
\hline Meghalaya & 0.190 & 0.395 & 0.162 & $37 \%$ & $41 \%$ & $14 \%$ & $15 \%$ & $18 \%$ & $8 \%$ & $18 \%$ & $5 \%$ & $8 \%$ & $6 \%$ & $4 \%$ & $10 \%$ & $9 \%$ \\
\hline Mizoram & 0.059 & 0.236 & 0.221 & $53 \%$ & $19 \%$ & $2 \%$ & $8 \%$ & $14 \%$ & $13 \%$ & $25 \%$ & $3 \%$ & $6 \%$ & $5 \%$ & $1 \%$ & $10 \%$ & $13 \%$ \\
\hline Nagaland & 0.154 & 0.348 & 0.171 & $46 \%$ & $40 \%$ & $9 \%$ & $12 \%$ & $16 \%$ & $10 \%$ & $16 \%$ & $4 \%$ & $8 \%$ & $6 \%$ & $9 \%$ & $11 \%$ & $9 \%$ \\
\hline Orissa & 0.213 & 0.420 & 0.134 & $32 \%$ & $49 \%$ & $14 \%$ & $12 \%$ & $9 \%$ & $12 \%$ & $21 \%$ & $7 \%$ & $10 \%$ & $3 \%$ & $8 \%$ & $10 \%$ & $8 \%$ \\
\hline Punjab & 0.074 & 0.250 & 0.225 & $57 \%$ & $22 \%$ & $3 \%$ & $11 \%$ & $15 \%$ & $13 \%$ & $24 \%$ & $1 \%$ & $10 \%$ & $0 \%$ & $8 \%$ & $12 \%$ & $5 \%$ \\
\hline Rajasthan & 0.216 & 0.426 & 0.132 & $31 \%$ & $47 \%$ & $16 \%$ & $11 \%$ & $14 \%$ & $14 \%$ & $21 \%$ & $5 \%$ & $9 \%$ & $4 \%$ & $6 \%$ & $10 \%$ & $7 \%$ \\
\hline Sikkim & 0.095 & 0.280 & 0.208 & $53 \%$ & $29 \%$ & $4 \%$ & $15 \%$ & $19 \%$ & $9 \%$ & $14 \%$ & $2 \%$ & $7 \%$ & $5 \%$ & $6 \%$ & $11 \%$ & $11 \%$ \\
\hline Tamil Nadu & 0.077 & 0.261 & 0.170 & $54 \%$ & $30 \%$ & $1 \%$ & $9 \%$ & $5 \%$ & $13 \%$ & $26 \%$ & $3 \%$ & $14 \%$ & $2 \%$ & $5 \%$ & $13 \%$ & $10 \%$ \\
\hline Tripura & 0.160 & 0.360 & 0.149 & $42 \%$ & $44 \%$ & $9 \%$ & $10 \%$ & $7 \%$ & $12 \%$ & $23 \%$ & $6 \%$ & $6 \%$ & $5 \%$ & $11 \%$ & $11 \%$ & $8 \%$ \\
\hline Uttar Pradesh & 0.230 & 0.446 & 0.109 & $27 \%$ & $52 \%$ & $16 \%$ & $9 \%$ & $14 \%$ & $16 \%$ & $20 \%$ & $7 \%$ & $9 \%$ & $1 \%$ & $8 \%$ & $10 \%$ & $6 \%$ \\
\hline
\end{tabular}


$\begin{array}{llllllllllllllllll}\text { Uttaranchal } & 0.109 & 0.317 & 0.148 & 43 \% & 36 \% & 4 \% & 7 \% & 8 \% & 13 \% & 26 \% & 4 \% & 10 \% & 3 \% & 9 \% & 12 \% & 8 \%\end{array}$

$\begin{array}{llllllllllllllllllllllll}\text { West Bengal } & 0.192 & 0.400 & 0.151 & 35 \% & 45 \% & 13 \% & 14 \% & 11 \% & 11 \% & 22 \% & 7 \% & 8 \% & 1 \% & 8 \% & 10 \% & 7 \%\end{array}$

\begin{tabular}{llllllllllllllllll} 
India & 0.179 & 0.386 & 0.160 & $37 \%$ & $42 \%$ & $12 \%$ & $11 \%$ & $12 \%$ & $13 \%$ & $22 \%$ & $6 \%$ & $9 \%$ & $2 \%$ & $7 \%$ & $10 \%$ & $7 \%$ \\
\hline
\end{tabular}

Table 1.04 illustrates this decomposition of the overall headcount ratio and the additional information that it provides. For instance, whereas the vast majority of Kerala's population is deprivation affected (73\%), the percentage of those who are severely poor is zero. In Bihar, on the other hand, the percentage of the deprivation affected is rather low (18\%) as the vast majority $(80 \%)$ are either poor $(52 \%)$ or severely poor $(28 \%)$. The differentiation augments the knowledge about the poverty structure in different regions and countries, a valuable property for policy makers.

The table also demonstrates the advantages of the index's additive structure, also especially relevant for policy makers: factor decomposability facilitates the decomposition of the CSPI according to the contribution of the different attributes to overall poverty. For instance, in Himachal Pradesh, the contribution of the health dimension is $44 \%$, other areas of concern include cooking fuel (15\%) and sanitation (14\%). In Sikkim, on the other hand, it is especially the education dimension that contributes to poverty (34\% compared to $8 \%$ in Himachal Pradesh). Thus, it would be advisable for policy-makers to design a different set of policies for each country; in Himachal Pradesh this would include special efforts to enhance the health care system, in Sikkim a stronger focus should be on investments in the education system.

However, the main advantage of the CSPI is the additional information that it provides with regard to inter-personal inequality. The benefits of this feature are best appreciated by means of regional poverty maps. 
Figure 1.04 Indian Poverty Maps according to MPI and CSPI
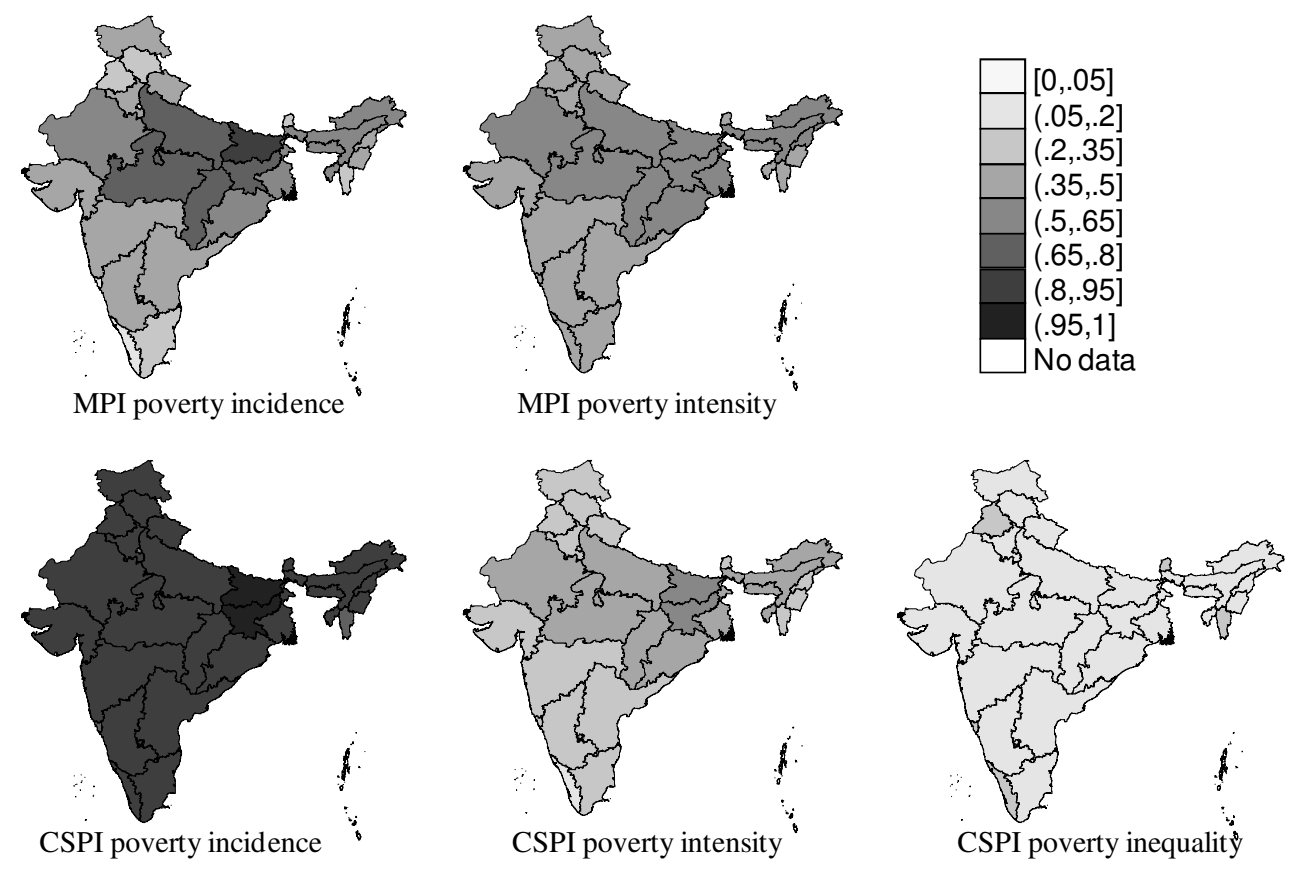

Figure 1.04 presents the regional poverty maps according to the decomposed MPI and CSPI, respectively. Obviously, the MPI is only able to provide poverty maps for poverty incidence and intensity whose explanatory power is in addition reduced as a result of the artificial cut-off. The CSPI, on the other hand, is able to provide a much more detailed and distinct picture of poverty. Without the MPIs sensitivity to an arbitrary cut-off, it is able to clearly identify the best and worst performers with regard to the different poverty components. The states demonstrating the highest levels of poverty incidence are Bihar and Jharkhand. Both states are also the ones with the highest level of poverty intensity, followed by Madhya Pradesh, Uttar Pradesh, Rajasthan and Orissa. Some of the best performers with regard to overall poverty, especially Kerala, but also Punjab, Mizoram and Sikkim, are the worst performers with regard to inequality. The detailed picture of poverty provided by the CSPI allows for more informed policy making; the removal of distortions and the sensitivity with regard to inter-personal inequality enhances targeting.

\subsection{Conclusions}

This chapter takes a more holistic approach to inequality between dimensions than currently done in the literature by defining it as the association-sensitive spread of simultaneous deprivations across the population, a definition that accounts for the two concepts of distributive justice and efficiency. It was demonstrated how the introduced axiomatic pendant 
to the expanded definition implies a new identification method that was used to derive a new class of poverty indices. This class is unique in the sense that it is the first class of additive poverty indices that is simultaneously i) sensitive to distributive justice considerations and therefore explicitly accounts for the fact that households may suffer from simultaneous deprivations, ii) sensitive to the relationship between attributes, thereby explicitly accounting for the concept of efficiency. As a result, it improves the precision and detailedness of poverty profiles, thereby enhancing the targeting of poverty reduction policies.

Obviously, this chapter leaves a lot of issues related to the topic of multidimensional poverty measurement untouched. It does not question the weights and poverty thresholds of the MPI. Also, the chapter follows the literature in assuming the same relationship between each pair of poverty attributes. Though there seems to be good reason to assume an independent relationship between poverty dimensions, the same does not hold in the case of attributes, especially for those displaying the same poverty dimension. A first step to take care of this problem has been introduced by Silber (2011) who suggested to first aggregate attributes into the respective poverty dimensions, taking care of their most likely dependent relationship, and only afterwards to aggregate the most likely independent dimensions themselves. This seems to be a promising approach for future research. Last but not least, the present chapter focuses only on ordinal poverty indices. The issue of cardinal poverty indices will be the subject of the following chapter. 


\section{Chapter 2}

\section{Efficiency and Distributive Justice in Cardinal Poverty Indices: The Inequality Sensitive Poverty Index}

As in the previous chapter, the focus of this chapter is on the two concepts of distributive justice and efficiency, only this time within the context of cardinal multidimensional poverty measurement. Within this framework, the concept of distributive justice is usually equated with the concept of efficiency, i.e. inequality between dimensions is usually equated with association-sensitivity. This chapter follows the argumentation of the previous one, suggesting to define inequality between dimensions as the association-sensitive spread of simultaneous deprivations across the population. As is in the ordinal framework, the more holistic approach to inequality between dimensions facilitates the introduction of a new axiom as well as the utilization of the same new identification method introduced in the previous chapter. As in chapter one, this approach provides the basis for a new class of multidimensional poverty indices. The latter is again unique in the sense that it is the first cardinal class of additive poverty indices that is able to take care of considerations of distributive justice and efficiency at the same time. A representative of this class is the Inequality Sensitive Poverty Index (ISPI) that is exemplarily applied to DHS data sets for a sample of 28 countries and again illustrates the empirical relevance of the new methodological approach.

\subsection{Introduction}

The fact that poverty is a multidimensional phenomenon is undisputed, even in the income poverty literature. In fact, income is not supposed to be important per se but rather to serve as an indicator for economic resources that enable individuals to satisfy their multidimensional needs. However, the suitability of insufficient income as indicator for poverty has been increasingly questioned (e.g. Rawls, 1971; Sen, 1985; Drèze and Sen, 1989; UNDP, 1995). Like the first, also this second chapter utilises a multidimensional approach to 
measure poverty, building on Amartya Sen's capability approach (Sen, 1985; 1992; 1997; 2009). The poor within a society are defined as those unable to achieve a minimum capability set of elementary functionings, like the ability to be well-nourished or to have access to education. Such a definition implies that the opportunity of the poor to develop their human capital and reach their full potential is limited by external circumstances, such as socioeconomic background, race, gender, religion, poor health, or malnutrition.

A direct consequence of this definition of poverty is that inequality among the poor is inequality in the inability to develop the own potential due to external circumstances, i.e. factors that are "beyond the scope of individual responsibility" (Marrero and Rodríguez, 2010, p.3). This explains why Amartya Sen (1976) so forcefully requires any reasonable poverty index to be sensitive to inequality: inequality among the poor is in fact inequality of opportunity that should be fought not only from a distributive justice perspective, but as well from an aggregate perspective as it wastes human capital and thus limits the overall expansion of capabilities in a society (Sen, 1992; Marrero and Rodríguez, 2010).

In a multidimensional framework, inequality persists in two forms: inequality within dimensions (Kolm, 1977) and inequality between dimensions (Atkinson and Bourguignon, 1982). Whereas the former is defined as the spread of distributions within poverty dimensions; the latter is usually treated as association sensitivity (e.g. Bourguignon and Chakravarty, 2003; Seth, 2011).

This chapter claims that the latter approach is inappropriate as it equates the two concepts distributive justice and efficiency which in reality are in tension, sometimes even opposed to one another. Inequality should not be reduced to an evaluation of how efficient poverty attributes are distributed among the poor but also consider who gains and who loses from redistributions. Therefore, the new property "Inequality Sensitivity (IS)" is introduced that basically requires poverty to increase (in the case of substitutes) or to decrease (in the case of complements) if an association increasing switch between two poor persons comes at the expense of the poorer of the two. ${ }^{17}$ It is demonstrated that the new axiom uniquely characterises a class of poverty indices that is actually the first that though additive is nevertheless able to account for both inequality within and between dimensions.

\footnotetext{
${ }^{17}$ I follow the Auspitz-Lieben-Edgeworth-Pareto (ALEP) definition of substitutability and complementarity. The ALEP definition considers two attributes to be substitutes if their second cross partial derivatives are positive. Intuitively, an increase in one attribute decreases poverty the less the higher the achievements in the second attribute. In the same way, attributes are considered to be complements, when the respective cross partial derivatives are negative and independent in case they are zero.
} 
The empirical implications are demonstrated for a sample of 28 developing countries for which three different indices are calculated: i) the $M_{0}$ of the Alkire and Foster class of indices (2011) that is insensitive to either type of inequality, ii) the multidimensional FGT class of indices that is sensitive to inequality within dimensions, and, finally, iii) the new class of inequality sensitive poverty indices $P_{I S}$ that, as the name suggests, is sensitive to within and between dimensional inequality. The relevance of the sensitivity requirement with regard to both types of inequality is easily established once the distinct changes in country rankings induced by the switch from one index to the next are investigated.

The chapter proceeds as follows. The second section provides a brief introduction in the theoretical background of the chapter. Section three lays the axiomatic foundation for the derivation and decomposition of the new class of indices in section four that are utilised in the empirical application presented in section five. Section six concludes. Proofs are relegated to the appendix.

\subsection{Theoretical Background}

Let $\mathbb{R}^{\mathrm{k}}$ denote the Euclidean $k$-space, and $\mathbb{R}_{+}^{\mathrm{k}} \subset \mathbb{R}^{\mathrm{k}}$ the non-negative $k$-space. Further, let $\mathbb{N}$ denote the set of positive integers. $\mathbf{N}=\{1, \ldots, n\} \subset \mathbb{N}$ represents the set of $n$ individuals of a typical society and $\mathbf{D}=\{2, \ldots, d\} \subset \mathbb{N}$ the set of $d$ poverty dimensions captured by a set of $k$ poverty attributes $\mathbf{K}=\{2, \ldots, k\} \subset \mathbb{N}$.

Let $\mathbf{a} \in \mathbb{R}_{+}^{\mathbf{K}}$ denote the weight vector for the different attributes with $\sum_{j=1}^{k} a_{j}=1$. In the following, I will refer to the quantity of an attribute with which an individual is endowed as an achievement. The achievement vector of individual $i$ is represented by $\mathbf{x}_{i \cdot}=\left(x_{i 1}, \ldots, x_{i k}\right)$ and the respective achievement matrix of a society with $n$ individuals by $\mathbf{X} \in \mathbb{R}_{+}^{\mathrm{NK}}$ where the $i j$ th entry represents the achievement $x_{i j}$ of individual $i$ in attribute $j$.

Let $X_{\mathrm{n}}$ be the set of possible achievement matrices of population size $n$ and $X=\mathrm{U}_{\mathbf{N}} \subset_{\mathbb{N}} X_{\mathrm{n}}$ the set of all possible achievement matrices. Let $z_{j}$ denote the poverty threshold of attribute $j$ so that individual $i$ is deprived in $j$ whenever the respective achievement falls short of the threshold level, i.e. whenever $x_{i j}<z_{j}$. Further, let $\mathbf{z} \in \mathbb{R}_{++}^{\mathbf{K}}$ represent the vector of poverty thresholds chosen for the different attributes, with the $j$ th element being $z_{j}$, and $\mathbf{Z}$ being the set of all possible vectors of poverty thresholds.

In the context of this chapter, a poverty index is a function $P: X \times \mathbf{Z} \rightarrow \mathbb{R}$. For any poverty threshold vector $\mathbf{z} \in \mathbf{Z}$, society $\mathcal{A}$ has a higher poverty level than society $\mathscr{B}$ if and only if $P\left(\mathbf{X}^{\mathcal{A}} ; \mathbf{z}\right) \geq P\left(\mathbf{X}^{\mathcal{B}} ; \mathbf{z}\right)$ for any $\mathbf{X}^{\mathcal{A}}, \mathbf{X}^{\mathcal{B}} \in X$. 
Let $\mathbf{c}_{i}=\left(c_{i 1}, \ldots, c_{i k}\right)$ represent the deprivation vector of individual $i$ such that $c_{i j}=1$ if $x_{i j}<z_{j}$ and $c_{i j}=0$ if $x_{i j} \geq z_{j}$. Further, let $S_{j}(\mathbf{X})$ - or simply $S_{j}$ - denote the set of individuals who are poor with respect to attribute $j$ and $q$ the overall number of poor individuals in a society.

For reasons of simplicity, let $\delta_{i}=\sum_{j \in\{1, \ldots, k,\}_{j j}=1} a_{j}$ denote the sum of weighted deprivations suffered by individual $i$, with $\mu(\boldsymbol{\delta})=1 / q \sum_{i \in S_{j}} \delta_{i}$. Also, let $g_{i j}=\left(1-x_{i j} / z_{j}\right)$ denote the poverty gap of individual $i$ and attribute $j$, with $\mu_{j}(\mathbf{g})=1 / q_{j} \sum_{i \in S_{j}} g_{i j}$.

Finally, let $\rho: \mathbb{R}_{+}^{\mathbf{K}} \times \mathbb{R}_{++}^{\mathbf{K}} \rightarrow\{0,1\}$ represent an identification function according to the component poverty line approach so that individual $i$ is poor if $\rho\left(\mathbf{c}_{i} ; \mathbf{z}\right)=1$ and not poor if $\rho\left(\mathbf{c}_{i} ; \mathbf{z}\right)=0$. The approach is theoretically founded in the strong focus axiom considering each poverty attribute as essential in the sense that compensation is impossible. ${ }^{18}$

Three specifications of the identification function have been suggested so far. The union method is based on the assumption that all attributes are perfect complements and thus that every deprived person is considered poor. The intersection method considers all attributes to be perfect substitutes and thus identifies only those individuals as poor who are deprived in every single attribute. Both approaches are extreme cases, repeatedly yielding poverty rates that are plainly inapplicable, being either far too high or far too low (Bérenger and Bresson, 2010; Alkire and Foster, 2011). The third identification method, the intermediate method, has been developed as a loophole, considering only those individuals as poor that are deprived in some pre-determined minimum level of weighted deprivations (Mack and Lindsay, 1985; Foster, 2009; Alkire and Foster, 2011), i.e.:

$$
\rho_{I M}\left(\mathbf{c}_{i} ; \mathbf{z}\right)=\left\{\begin{array}{lll}
1 & \text { if } & \delta_{i} \geq \delta_{I M}^{\min } \\
0 & \text { if } & \delta_{i}<\delta_{I M}^{\min }
\end{array}\right.
$$

Please note that the intermediate method comprises union and intersection method as extreme cases, i.e. in case $\delta_{I M}^{\min } \hat{=} \max \left\{\mathbf{c}_{i .}\right\}=1$ and $\delta_{I M}^{\min } \hat{=} \min \left\{\mathbf{c}_{i .}\right\}=1$, respectively.

Though the intermediate method is a convenient way out of the dilemma of extreme poverty rates, its theoretical justification is questionable. Apart from the fact that the choice of $\delta_{I M}^{\min }$ is arbitrary, the whole method is based on the indirect assumption that up to $\delta_{I M}^{\min }$ attributes are perfect substitutes whereas they are considered perfect complements from $\delta_{I M}^{\mathrm{min}}$ onwards. In response, the first chapter introduced a new identification method that leads to

\footnotetext{
${ }^{18}$ The other main method for the identification of the poor is called aggregate poverty line approach. The special feature of this method is that it allows compensation between attributes below and above threshold levels among those who are poor (Weak Focus Axiom).
} 
applicable poverty rates and is theoretically founded in the concept of inequality between dimensions.

The new identification method is based on a multi- instead of a single step identification function:

$$
\rho_{C S}\left(\mathbf{c}_{i \cdot} ; \mathbf{z}\right)=\left\{\begin{array}{cll}
h\left(\mathbf{c}_{i \cdot}\right) & \text { if } & \max \left\{\mathbf{c}_{i \cdot}\right\}=1 \\
0 & \text { if } & \max \left\{\mathbf{c}_{i \cdot}\right\}=0
\end{array}\right.
$$

$h: \mathbb{R}_{+}^{\mathbf{K}} \rightarrow[0,1]$ being a function of poverty severity that is nondecreasing with a nondecreasing (nonincreasing) marginal ${ }^{19}$ in case attributes are substitutes (complements).

In other words, $\rho_{c s}\left(\mathbf{c}_{i} ; \mathbf{z}\right)$ is in a way a fuzzy approach that differentiates between the poor on one hand and different degrees of poverty severity on the other. The specific shape of the function is of intriguing simplicity: the function is always non-decreasing in the number of deprivations, however, the marginal increase in poverty severity is the less the higher the substitutability between attributes. The former accounts for distributive justice, the latter for efficiency. The relationship between the two is determined by alpha.

Alpha is an indicator for inequality aversion that is inextricably linked with the relationship among attributes and vice versa. More precise, a higher level of substitutability among attributes inevitably implies a higher level of inequality aversion and vice versa.

Figure 2.01 The Correlation Sensitive Identification Method

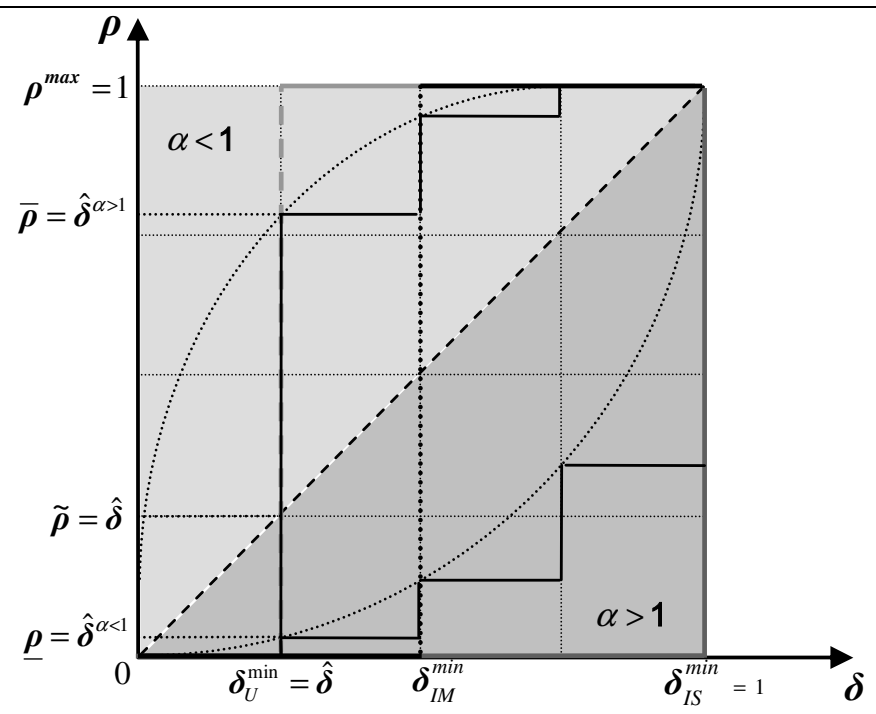

Figure 2.01 demonstrates the functioning of the new identification method: instead of differentiating between the poor and the non-poor, the new function differentiates between the non-poor on one hand and different degrees of poverty severity on the other.

\footnotetext{
${ }^{19}$ A function $f(x)$ has a nondecreasing marginal if $f\left(x_{g}+1\right)-f\left(x_{g}\right) \geq f\left(x_{h}+1\right)-f\left(x_{h}\right)$ whenever $x_{g} \geq x_{h}$.
} 
In case $\alpha<1, \rho_{c s}\left(\mathbf{c}_{i} ; \mathbf{z}\right)$ approximates a concave shape, i.e. the increase in the level of poverty severity is marginally decreasing in the number of deprivations as the loss in even one attribute can hardly be compensated. This, in turn, implies a low level of inequality aversion: as already the loss in one attribute can barely be compensated, there is in fact no need for a strong focus on inequality. This is expressed by a low value of alpha.

In case $\alpha>1, \rho_{c s}\left(\mathbf{c}_{i} ; \mathbf{z}\right)$ approximates a convex shape, i.e. the increase in the level of poverty severity is marginally increasing in the number of deprivations: the lack in only one attribute leads to a rather low poverty degree as $k-1$ other attributes can compensate for the loss. Obviously, there is a need for a strong focus on inequality as expressed by a high value of alpha.

This way, the new identification method accounts for possible association sensitivity among attributes through the specific shape of the function: while it is always nondecreasing in the number of deprivations, the marginal increase in poverty severity is the less the higher the substitutability between attributes.

\subsection{The Axiomatic Foundation for Cardinal Poverty Measures}

Four main aggregation methods have been developed in order to derive a composite index from individual poverty characteristics: i) the fuzzy set approach, ii) the distance function approach, iii) the information theory approach, and iv) the axiomatic approach (see Deutsch and Silber 2005). Based on the same argumentation as in chapter one, I refrain from applying the former two as they do not allow for an attribute-wise consideration of poverty. The information theory approach has recently been extended to cover the component poverty line approach (Maasoumi and Lugo 2008). Its special appeal stems from the fact that it summarizes the information inherent in all attributes in an efficient manner. Nevertheless, the argumentation of this chapter is that inequality is not only a concept of efficiency but also includes normative judgments as captured by the concept of distributive justice. The axiomatic approach provides the most transparent way to take care of these judgments by explicitly defining properties that poverty indices may or may not satisfy. However, as has been pointed out in the previous chapter, the information theory approach can be utilized as an add-on in the sense that all classes of poverty measures can be tested on whether they satisfy the efficiency criteria of the information theory approach or not. This way, both approaches can be combined, using the best of both of them. 
This section starts with a brief overview of the list of core axioms (e.g. Chakravarty, Mukherjee and Ranade 1998, Bourguignon and Chakravarty 1999, Tsui 2002, Bourguignon and Chakravarty 2003, Chakravarty and Silber 2008) that were already introduced in the previous chapter.

\section{Non-Distributional Axioms}

Anonymity (AN): For any $\mathbf{z} \in Z$ and $\mathbf{X} \in X_{\mathrm{n}}, P(\mathbf{X} ; \mathbf{z})=P(\boldsymbol{\Pi X} ; \mathbf{z})$ where $\boldsymbol{\Pi}$ is any permutation matrix of appropriate order.

Continuity $(\mathbf{C N})$ : For any $\mathbf{z} \in Z$ and $\mathbf{X} \in X_{\mathrm{n}}, P(\mathbf{X} ; \mathbf{z})$ is continuous on $\mathbb{R}_{+}^{\mathrm{NK}}$.

Monotonicity (MN): For any $\mathbf{z} \in Z$ and $\mathbf{X}, \mathbf{X}^{\prime} \in X_{\mathrm{n}}$, if for any individual $h$ and any attribute $l$ $x_{h l}=x_{h l}^{\prime}+\beta, \quad$ such $\quad$ that $\quad x_{h l}^{\prime}<z_{l}, \beta>0$, and $\quad x_{i l}=x_{i l}^{\prime} \forall i \neq h, \quad x_{i j}=x_{i j}^{\prime} \forall j \neq l, \forall i$, then $P\left(\mathbf{X}^{\prime} ; \mathbf{z}\right) \leq P(\mathbf{X} ; \mathbf{z})$.

Principle of Population (PP): If for any $\mathbf{z} \in Z, \mathbf{X} \in X_{\mathrm{n}}$, and $m \in \mathbb{N} \mathbf{X}^{m}$ is a $m$-fold replication of $\mathbf{X}$, then $P\left(\mathbf{X}^{m} ; \mathbf{z}\right)=P(\mathbf{X} ; \mathbf{z})$.

Strong Focus (SF): For any $\mathbf{z} \in Z$ and $\mathbf{X} \in X_{\mathrm{n}}$, if for any individual $h$ and any attribute $l$ $x_{h l} \geq z_{l}, x_{h l}^{\prime}=x_{h l}+\beta, \beta>0$, and $x_{i l}^{\prime}=x_{i l} \forall i \neq h, x_{i j}^{\prime}=x_{i j} \forall j \neq l, \forall i$, then $P(\mathbf{X} ; \mathbf{z})=P\left(\mathbf{X}^{\prime} ; \mathbf{z}\right)$.

Subgroup Decomposability (SD): For any $\mathbf{X}^{1}, \ldots, \mathbf{X}^{v} \in X_{\mathrm{n}}$ and $\mathbf{z} \in Z$, $P\left(\mathbf{X}^{1}, \mathbf{X}^{2}, \ldots, \mathbf{X}^{v} ; \mathbf{z}\right)=\sum_{l=1}^{v} n_{l} / n P\left(\mathbf{X}^{l} ; \mathbf{z}\right)$ with $n_{l}$ being the population size of subgroup $\mathbf{X}^{l}, l=1, \ldots, v$ and $\sum_{l=1}^{v} n_{l}=n$.

Factor Decomposability (FD): For any $\mathbf{z} \in Z$ and $\mathbf{X} \in X_{\mathrm{n}}, P(\mathbf{X} ; \mathbf{z})=\sum_{j=1}^{k} a_{j} P\left(x_{. j} ; z_{j}\right)$

Normalization (NM): For any $\mathbf{z} \in Z$ and $\mathbf{X} \in X_{\mathrm{n}}, P(\mathbf{X} ; \mathbf{z})=1$ if $x_{i j}=0 \forall i, j$ and $P(\mathbf{X} ; \mathbf{z})=0$ if $x_{i j} \geq z_{j} \forall i, j$. Thus, $P(\mathbf{X} ; \mathbf{z}) \in[0,1]$.

AN requires that any personal characteristics apart from the respective achievement levels are irrelevant for poverty measurement. $\mathrm{CN}$ is a rather technical requirement precluding the oversensitivity of poverty measures. MN requires poverty measures not to increase if, ceteris paribus, the condition of a deprived individual improves. PP precludes the dependence of poverty measures from population size and thus allows for cross-population and -time comparisons of poverty. SF demands that giving a person more of an attribute with respect to which this person is not deprived will not change the poverty measure. FD and SD facilitate the calculation of the contribution of different subgroup-attribute combinations to overall poverty, improving the targeting of poverty-alleviating policies. NM is a simple technical 
property requiring poverty measures to be equal to zero in case all individuals are non-poor and equal to one in case all individuals are poor.

\section{Distributional Axioms}

I will now turn to the group of axioms that specifically deal with inequality issues. Scale Invariance (SI) requires that a proportional distribution should leave inequality levels unchanged, ensuring that poverty indices do not change with the unit of measurement.

Scale Invariance (SI): For any $\mathbf{z} \in Z$ and $\mathbf{X}, \mathbf{X}^{\prime} \in X_{\mathrm{n}}, P(\mathbf{X} ; \mathbf{z})=P\left(\mathbf{X}^{\prime} ; \mathbf{z}^{\prime}\right)$ where $\mathbf{X}^{\prime}=\mathbf{X} \mathbf{\Lambda}$; $\mathbf{z}^{\prime}=\Lambda \mathbf{z}$ with $\boldsymbol{\Lambda}$ being the diagonal matrix $\operatorname{diag}\left(\lambda_{1}, \ldots, \lambda_{k}\right), \lambda_{j}>0 \forall j$.

In order to capture inequality within dimensions, poverty should not decrease in case the spread of dimension-specific achievements across society increases. In the one-dimensional context, this property is referred to as the Pigou-Dalton Transfer Principle. Different mathematical formulas have been used to extent the property to the multidimensional framework (de la Vega, Urrutia and de Sarachu, 2010). The one most widely used is the Uniform Majorization (UM) axiom.

Uniform Majorization (UM): For any $\mathbf{z} \in Z$ and $\mathbf{X}, \mathbf{X}^{\prime} \in X_{\mathrm{n}}$, if $\mathbf{X}^{P}=\mathbf{B} \mathbf{X}^{\prime P}$ and $\mathbf{B}$ is not a permutation matrix, then $P(\mathbf{X} ; \mathbf{z}) \leq P\left(\mathbf{X}^{\prime} ; \mathbf{z}\right)$, where $\mathbf{X}^{P}\left(\mathbf{X}^{\prime P}\right)$ is the attribute matrix of the poor corresponding to $\mathbf{X}\left(\mathbf{X}^{\prime}\right)$ and $\mathbf{B}=\left(b_{i j}\right)$ is some bistochastic matrix of appropriate order.

UM requires that a transformation of the attribute matrix $\mathbf{X}^{\prime P}$ of the poor in $\mathbf{X}^{\prime}$ into the corresponding matrix $\mathbf{X}^{P}$ of the poor in $\mathbf{X}$ by an equalising operation does not increase poverty.

As has been pointed out, in a multidimensional framework exists yet another aspect of inequality, namely inequality between poverty dimensions. This type of inequality has traditionally been equated with association sensitivity and captured by the concept of an association increasing switch. ${ }^{20}$ The underlying majorization criterion has been proposed by Boland and Proschan (1988) and was generalized and formally introduced by Tsui (1999) as "Correlation Increasing Transfer".

Association Increasing Switch: ${ }^{21}$ For any two vectors $\mathbf{x}=\left(x_{1}, \ldots, x_{k}\right)$ and $\mathbf{x}^{\prime}=\left(x_{1}^{\prime}, \ldots, x_{k}^{\prime}\right)$ define the two operators $\bar{\lambda}$ and $\bar{v}$ as follows: $\mathbf{x} \bar{\wedge} \mathbf{x}^{\prime}=\left(\min \left\{x_{1}, x_{1}^{\prime}\right\}, \ldots, \min \left\{x_{k}, x_{k}^{\prime}\right\}\right)$ and

\footnotetext{
${ }^{20}$ Based on a paper of Chakravarty and D'Ambrosio (2006) on social exclusion measures, Jayaraj and Subramanian (2010) introduce inequality between dimensions as the spread of simultaneous deprivations across a society and based on this definition formulate the property '(Strong) Range Sensitivity'. However, the authors fail to account for association-sensitivity which is why this chapter refrains from employing these properties.

${ }^{21}$ Please note that the concept of the 'Association Increasing Switch' is slightly different from the 'Correlation Increasing Switch' formulated by Bourguignon and Chakravarty (2003). The latter definition is unclear as it
} 
$\mathbf{x} \bar{\nabla} \mathbf{x}^{\prime}=\left(\max \left\{x_{1}, x_{1}^{\prime}\right\}, \ldots, \max \left\{x_{k}, x_{k}^{\prime}\right\}\right)$. For every $\mathbf{X}, \mathbf{X}^{\prime} \in X_{\mathrm{n}}, \mathbf{X}^{\prime}$ is obtained from $\mathbf{X}$ by an association increasing switch if $\mathbf{X}^{\prime}$ is not a permutation of $\mathbf{X}$ and if for some poor individuals $g$ and $h, \mathbf{x}_{g}^{\prime}=\mathbf{x}_{g} \bar{\wedge} \mathbf{x}_{h}, \mathbf{x}_{h}^{\prime}=\mathbf{x}_{g} \bar{\nabla} \mathbf{x}_{h}$ and $\mathbf{x}_{m}^{\prime}=\mathbf{x}_{m} \forall m \notin\{g, h\}$.

Consider two persons who - though both of them deprived in all attributes - face different achievement levels: each person has less than the other of at least one attribute. A switch of achievements is called association increasing if, after the switch, one of the two persons has at least as much as the other of all attributes.

For the purpose of illustration consider the following situation of three individuals and four attributes:

$$
i=3, j=4, \mathbf{z}=\left(\begin{array}{llll}
5 & 5 & 5 & 5
\end{array}\right) \text { and } \mathbf{X}=\left[\begin{array}{llll}
1 & 2 & 4 & 4 \\
4 & 1 & 2 & 2 \\
2 & 4 & 1 & 1
\end{array}\right] \text {. }
$$

Now, consider the following switches of achievements, first between individual one and individuals two and three, afterwards between individual two and three:

$$
\mathbf{X}=\left[\begin{array}{llll}
1 & 2 & 4 & 4 \\
4 & 1 & 2 & 2 \\
2 & 4 & 1 & 1
\end{array}\right] \rightarrow\left[\begin{array}{llll}
4 & 4 & 4 & 4 \\
1 & 1 & 2 & 2 \\
2 & 2 & 1 & 1
\end{array}\right] \rightarrow\left[\begin{array}{llll}
4 & 4 & 4 & 4 \\
2 & 2 & 2 & 2 \\
1 & 1 & 1 & 1
\end{array}\right]
$$

Through the switches, individual one receives strictly higher, individual three strictly lower achievements in all attributes. Thus, the switches lead to a concentration of attributes and thus higher inequality. Based on the concept of association increasing switches, Tsui (1999) introduced the following property.

Nondecreasingness under Association Increasing Switch (NDA): For any $\mathbf{X}, \mathbf{X}^{\prime} \in X_{\mathrm{n}}$ such that $\mathbf{X}^{\prime}$ is obtained from $\mathbf{X}$ by an association increasing switch of substitute attributes, $P(\mathbf{X} ; \mathbf{z}) \leq P\left(\mathbf{X}^{\prime} ; \mathbf{z}\right)$.

Bourguignon and Chakravarty (2003), however, claimed that in case attributes are complements, poverty should decrease even though association increasing switches lead to an increase in within dimensional inequality. In response, they introduce the following additional property.

Nonincreasingness under Association Increasing Switch (NIA): For any $\mathbf{X}, \mathbf{X}^{\prime} \in X_{\mathrm{n}}$ such that $\mathbf{X}^{\prime}$ is obtained from $\mathbf{X}$ by an association increasing switch of complement attributes, $P(\mathbf{X} ; \mathbf{z}) \geq P\left(\mathbf{X}^{\prime} ; \mathbf{z}\right)$.

requires an increase in the correlation between two attributes but leaves the correlation between all other attributes unaltered. 
For the purpose of illustration consider left and right shoes to be the poverty attributes in question. Obviously, the two attributes are complements; a right shoe is only valuable in case it comes along with a left shoe to make it a pair. Let's assume an economy with two poor individuals and given poverty thresholds of ten left and ten right shoes per persons. Further, let one person have an initial endowment of eight left and two right shoes and the other an initial endowment of one left and three right shoes:

$$
i=2, j=2, \mathbf{z}=\left(\begin{array}{ll}
10 & 10
\end{array}\right) \text { and } \mathbf{X}=\left[\begin{array}{ll}
8 & 2 \\
1 & 3
\end{array}\right] \text {. }
$$

In other words, person one faces a surplus of six left shoes, person two a surplus of two right shoes. Obviously, the situation is not efficient. Indeed, two association increasing switches are possible that would enhance the overall situation. In the first one, the persons would exchange their amount of left shoes, i.e.:

$$
\mathbf{X}^{\prime}=\left[\begin{array}{ll}
1 & 2 \\
8 & 3
\end{array}\right]
$$

in the second their amount of right shoes, i.e.:

$$
\mathbf{X}^{\prime \prime}=\left[\begin{array}{ll}
8 & 3 \\
1 & 2
\end{array}\right] \text {. }
$$

Figure 2.02 Pareto-Efficiency and Association Increasing Switches

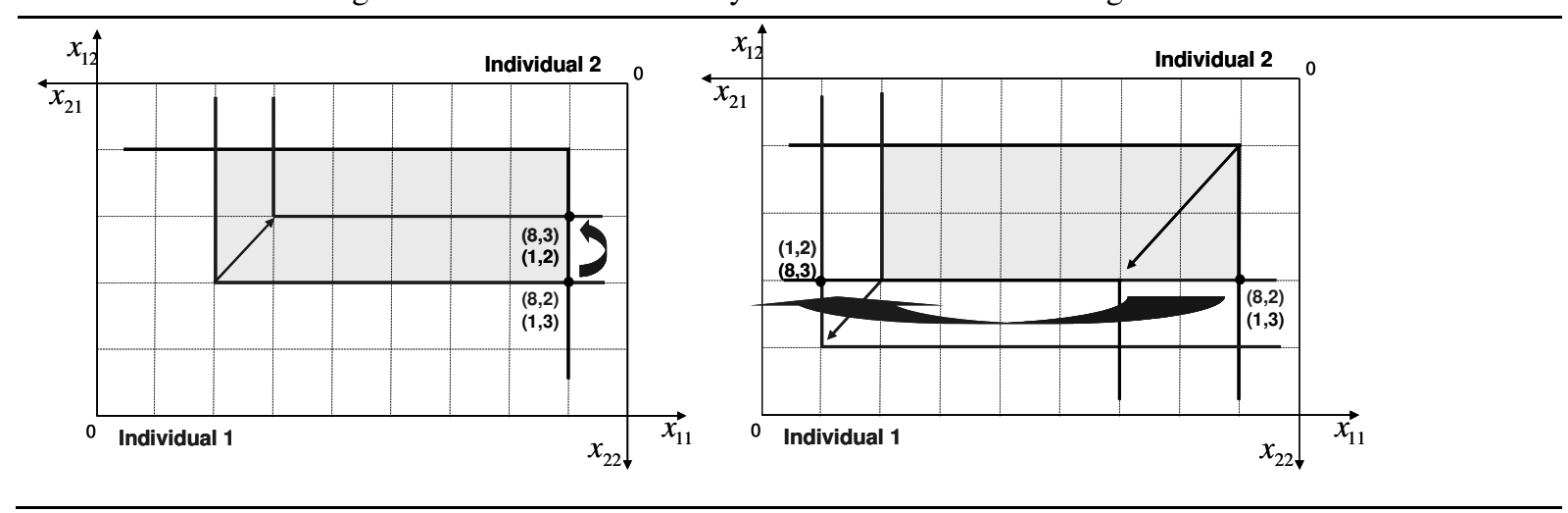

Both situations should be preferred to the initial one as an additional pair of shoes is made available. This is exactly the consideration behind NIA.

But there is an important difference between the two switches that is illustrated in figure 2.02. Under the first switch, the second person gains two additional pairs of shoes, the first person, however, actually looses one pair. Under the second switch, the first person gains an additional pair of shoes whereas the situation of the second person remains unchanged. Though the overall outcome is the same, one person possessing one and the other three pairs of shoes, the processes that led to the respective outcomes are different. Whereas the first 
switch would be strongly opposed by the first person, the second switch would encounter much less opposition as it improves the situation of one person without worsening the situation of the other, a characteristic that has become known in economic theory as paretoefficiency. For reasons that are obvious, pareto-efficiency is a rather valuable property for policy-makers. In the case of complements, pareto-efficiency can always be ensured if switches are restricted to cases in which the respective minimum achievement levels are not undercut. Thus, I extend the property NIA to ensure pareto-efficiency.

Nonincreasingness under Pareto-efficient Association Increasing Switch (NIPA): For any $\mathbf{X}, \mathbf{X}^{\prime} \in X_{\mathrm{n}}$ such that $\mathbf{X}^{\prime}$ is obtained from $\mathbf{X}$ by an association increasing switch of complement attributes between two poor individuals $g$ and $h$ with $\min \left\{\mathbf{x}_{g}\right\} \leq \min \left\{\mathbf{x}_{h}\right\}$ and $\mathbf{x}_{g}^{\prime}=\mathbf{x}_{g} \bar{\wedge} \mathbf{x}_{h}, \mathbf{x}_{h}^{\prime}=\mathbf{x}_{g} \bar{\nabla} \mathbf{x}_{h}$ and $\mathbf{x}_{m}^{\prime}=\mathbf{x}_{m} \forall m \notin\{g, h\}$, then $P(\mathbf{X} ; \mathbf{z}) \geq P\left(\mathbf{X}^{\prime} ; \mathbf{z}\right)$.

In case all individuals are deprived in all dimensions, sensitivity to (pareto-efficient) association increasing switches in connection with UM accounts satisfactory for both inequality within and between dimensions.

But what if individuals suffer from different numbers of simultaneous deprivations? This is a more than legitimate question, especially since this case serves as the main justification for poverty measures that go beyond simple averages.

Consider the following situation:

$$
i=2, j=5, \mathbf{z}=\left(\begin{array}{llll}
5 & 5 & 5 & 5
\end{array}\right) \text { and } \mathbf{X}=\left[\begin{array}{llll}
1 & 2 & 5 & 5 \\
2 & 1 & 5 & 1
\end{array}\right]
$$

and the following two possible switches:

$$
\mathbf{X}^{\prime}=\left[\begin{array}{llll}
2 & 2 & 5 & 5 \\
1 & 1 & 5 & 1
\end{array}\right] ; \mathbf{X}^{\prime \prime}=\left[\begin{array}{llll}
1 & 1 & 5 & 5 \\
2 & 2 & 5 & 1
\end{array}\right]
$$

Both switches constitute a weaker version of the original association increasing switches as they are not limited to persons who are deprived in all attributes. Instead, switches among persons who are deprived in different numbers of attributes are allowed as long as the respective switches concern only attributes in which all persons affected by the switch are deprived. Thus, in the example above, the focus would be on the first two attributes.

This chapter suggests that it is impossible to formulate any reasonable property that is based on a switch from $\mathbf{X}$ to either $\mathbf{X}^{\prime}$ or $\mathbf{X}^{\prime \prime}$. The reason is that such a general property would be obliged to include in some way value judgments that weight the severity of inequality within against inequality between dimensions. As we will see later on, the new class of poverty indices derived in this chapter captures this specific aspect with an interaction term. 
A general assessment, however, can be made with regard to the question who - given the association increasing switch takes place - should be the beneficiary of the switch, i.e. should the switch to $\mathbf{X}^{\prime}$ or $\mathbf{X}^{\prime \prime}$ be preferred? I suggest that the response to that question depends on the relationship between attributes. In case attributes are substitutes, the beneficiary of the switch should be the individual that is deprived in more attributes. In the example above, that would be $\mathbf{X}^{\prime \prime}$ as the beneficiary of the switch is the second individual that is deprived in three attributes instead of two. However, in case attributes are complements, pareto-efficient switches should be preferred, i.e. the individual with the higher minimum achievement level should be the beneficiary of the switch. In the example, that would be $\mathbf{X}^{\prime}$ as the second individual has only one unit of the fourth attribute and therefore no use for any additional amount of attribute one or two. In response, I introduce the following concept of an extended version of the association increasing switch and, based on that definition, a new property called Inequality Sensitivity (IS).

Weak Association Increasing Switch: Define $d_{i}=\#\left\{c_{i j} \mid c_{i j}=1\right\}$. For any two vectors $\mathbf{x}=\left(x_{1}, \ldots, x_{k}\right)$ and $\mathbf{x}^{\prime}=\left(x_{1}^{\prime}, \ldots, x_{k}^{\prime}\right)$ define the two operators $\stackrel{\wedge}{\wedge}$ and $\bar{\vee}$ as follows:

$$
\begin{aligned}
& \mathbf{x} \wedge \mathbf{x}^{\prime}=\left(\min \left\{x_{1}, x_{1}^{\prime}\right\}, \ldots, \min \left\{x_{k}, x_{k}^{\prime}\right\} \forall x_{j}<z_{j} ; x_{j}=x_{j}^{\prime} \forall x_{j} \geq z_{j}\right) \text { and } \\
& \mathbf{x} \vee \mathbf{x}^{\prime}=\left(\max \left\{x_{1}, x_{1}^{\prime}\right\}, \ldots, \max \left\{x_{k}, x_{k}^{\prime}\right\} \forall x_{j}<z_{j} ; x_{j}=x_{j}^{\prime} \forall x_{j} \geq z_{j}\right) .
\end{aligned}
$$

For every $\mathbf{X}, \mathbf{X}^{\prime} \in X_{\mathrm{n}}, \mathbf{X}^{\prime}$ is obtained from $\mathbf{X}$ by a weak association increasing switch if $\mathbf{X}^{\prime}$ is not a permutation of $\mathbf{X}$ and if for some poor individuals $g$ and $h, \mathbf{x}_{g}^{\prime}=\mathbf{x}_{g} \stackrel{=}{\wedge} \mathbf{x}_{h}$, $\mathbf{x}_{h}^{\prime}=\mathbf{x}_{g}=\bar{\nabla} \mathbf{x}_{h}$ and $\mathbf{x}_{m}^{\prime}=\mathbf{x}_{m} \forall m \notin\{g, h\}$.

Inequality Sensitivity (IS): Define $d_{i}=\#\left\{c_{i j} \mid c_{i j}=1\right\}$. For some $\mathbf{X}, \mathbf{X}^{\prime}, \mathbf{X}^{\prime \prime} \in X_{\mathrm{n}}$, if $\mathbf{X}^{\prime}$ and $\mathbf{X}^{\prime \prime}$ are obtained from $\mathbf{X}$ by a weak association increasing switch between two poor individuals $g$ and $h$ with $d_{g}>d_{h}>1$ such that:

$$
\begin{gathered}
\mathbf{x}_{g}^{\prime}=\mathbf{x}_{g} \wedge \mathbf{x}_{h}, \mathbf{x}_{h}^{\prime}=\mathbf{x}_{g} \vee \bar{\vee} \mathbf{x}_{h} \text { and } \mathbf{x}_{m}^{\prime}=\mathbf{x}_{m} \text { for all } m \notin\{g, h\} \text { and } \\
=\mathbf{x}_{g}^{\prime \prime}=\mathbf{x}_{g} \vee \mathbf{x}_{h}, \mathbf{x}_{h}^{\prime \prime}=\mathbf{x}_{g} \wedge \mathbf{x}_{h} \text { and } \mathbf{x}_{m}^{\prime \prime}=\mathbf{x}_{m} \text { for all } m \notin\{g, h\},
\end{gathered}
$$

then in case attributes are substitutes $P\left(\mathbf{X}^{\prime \prime} ; \mathbf{z}\right) \leq P\left(\mathbf{X}^{\prime} ; \mathbf{z}\right)$; in case attributes are complements, $P\left(\mathbf{X}^{\prime \prime} ; \mathbf{z}\right) \leq P\left(\mathbf{X}^{\prime} ; \mathbf{z}\right)$ if and only if $\min \left\{\mathbf{x}_{g}^{\prime \prime}\right\} \geq \min \left\{\mathbf{x}_{h}^{\prime \prime}\right\}$.

The concept of inequality increasing switches illustrates the previous observation that inequality between dimensions is closely related to the relationship between attributes yet not the same. The central theme of the following section is the derivation and comparison of poverty indices satisfying different levels of sensitivity to inequality within and between dimensions. 


\subsection{Inequality-Sensitive Poverty Indices}

Property 1. A multidimensional poverty measure P satisfies AN, CN, NM, MN, SF, PP, FD, $\mathrm{SD}, \mathrm{SI}, \mathrm{UM}$ and IS if and only if for all $n \in \mathbf{N}$ and $\mathbf{X} \in X_{\mathrm{n}}$ :

$$
P(\mathbf{X} ; \mathbf{z})=1 / n \sum_{i \in S_{j}} h\left(\mathbf{c}_{i}\right) \sum_{j=1}^{k} a_{j} f\left(x_{i j} / z_{j}\right)
$$

with $f:[0, \infty] \rightarrow R^{1}$ continuous, non-increasing and convex, with $f(0)=1$ and $f(t)=c$ for all $t \geq 1$ where $c<1$ is a constant. Also, $a_{j}>0$ are constants with $\sum_{j=1}^{k} a_{j}=1$.

Finally, $h: \mathbb{R}_{+}^{\mathbf{K}} \rightarrow[0,1]$ is nondecreasing with a nondecreasing (nonincreasing) marginal in case attributes are substitutes (complements).

The additive structure of the poverty measure is mandatory for the fulfilment of FD and automatically precludes sensitivity to association increasing switches. It also implies that sensitivity to inequality between dimensions can only be integrated in the final index through an adaptation in the identification step, as has been argued in the previous chapter.

The aggregation of individual poverty characteristics into the overall index should comprise i) normative judgements in order to guide the allocation of scarce resources to the most needy, and ii) efficiency considerations in order to ensure that no scarce resources get wasted. The functional forms of $h(\cdot)$ and $f(\cdot)$ should ensure both.

$h\left(\mathbf{c}_{i}\right)$ is derived from the identification function $\rho_{C S}: \mathbb{R}_{+}^{\mathbf{K}} \times \mathbb{R}_{++}^{\mathbf{K}} \rightarrow[0,1]$ that differentiates individuals according to the severity with which they suffer deprivation, thereby ensuring that the neediest receive appropriate attention. In the following, I will concentrate on the following specific functional form of $h\left(\mathbf{c}_{i}\right)$ that has been chosen due to its appealing intuitive and simple design:

$$
h\left(\mathbf{c}_{i}\right)=\left\{\begin{array}{lll}
\delta_{i}^{\alpha} & \text { if } & \max \left\{\mathbf{c}_{i}\right\}=1 \\
0 & \text { if } & \max \left\{\mathbf{c}_{i}\right\}=0
\end{array}\right.
$$

In other words, the degree of poverty severity is measured by the sum of weighted deprivations to the power $\alpha$. The parameter $\alpha$ can be interpreted as an indicator for aversion towards inequality between dimensions, the value of which ought to depend on the relationship among attributes. In fact, choosing a value for $\alpha$ that is smaller than one directly implies the assumption that attributes are complements, enforcing a concave shape of $h\left(\mathbf{c}_{i}\right)$. In this specific case, inequality between dimensions would actually be preferred, very much in the same sense as the intuition behind NIPA and IS. Choosing a value for $\alpha$ that is greater than one, on the other hand, directly determines a substitute relationship between attributes, enforcing a convex shape of $h\left(\mathbf{c}_{i}\right)$. 
Several suggestions have been made with regard to the functional form of $f(\cdot)$. However, as already noted, it seems that the axiomatic approach with its normative judgements does well in aggregating across individuals but not across attributes. Thus, I utilise the following "optimal" IT aggregation functions to ensure that attributes are aggregated in an efficient manner, wasting no scarce resources (Maasoumi and Lugo, 2008):

$$
\begin{gathered}
S_{i} \propto\left[\sum_{j=1}^{k} w_{j} v_{i j}^{\lambda}\right]^{1 / \lambda} \text { when } \lambda \neq 0 \\
S_{i} \propto \prod_{j=1}^{k} v_{i j}^{w_{j}} \text { when } \lambda=0
\end{gathered}
$$

$w_{j}$ being the weight attached to the Generalized Entropy divergence from each attribute. Please note that the optimal IT aggregation function imposes a union definition of poverty in the sense that all information about the distribution of attributes is taken care of (Maasoumi and Lugo 2008, p. 10).

Utilising the component poverty approach, the following family of IT-efficient multidimensional poverty indices can be derived as the $\alpha$ th moment of the distribution $\mathbf{S}_{v}=\left(S_{v 1}, \ldots, S_{v n}\right)$ :

$$
P\left(\mathbf{S}_{v} ; \mathbf{z}\right)=1 / n \sum_{i \in S_{j}} S_{v i}^{\gamma}
$$

with $S_{v i}$ representing the respective relative deprivation function according to (2.05) and (2.06).

In the following, I will introduce five of the most well-known (cardinal) classes of multidimensional poverty measures and discuss them under the aspect of IT-efficiency.

The first three classes have an additive structure and therefore lend themselves as functional forms for $f(\cdot)$ as specified in (1). The last two are non-additive.

\section{The multidimensional Foster-Greer-Thorbecke (FGT) class of poverty measures}

This class of poverty measures is a multidimensional extension of the Foster-GreerThorbecke index from 1984. The class is IT-efficient; the IT measure for $\delta \neq 0$ as specified in (2) is a version of this class of poverty measures in case $\gamma=\delta$ and $v_{i j}=1-x_{i j} / z_{j}$.

$$
P_{F G T}=1 / n \sum_{i \in S_{j}} \sum_{j \in\{1, \ldots, k\} c_{i j}=1} a_{j}\left(1-x_{i j} / z_{j}\right)^{\theta}
$$

with $a_{j}>0 ; \sum_{j=1}^{k} a_{j}=1 ; \theta>1$

Like $\alpha, \theta$ can be interpreted as an indicator for aversion towards inequality within dimensions. However, different from $\alpha, \theta$ is limited to values greater than one, reflecting the fact that it measures the aversion against inequality within every single dimension separately. 


\section{The first multidimensional Chakravarty class of poverty measures}

This class of poverty measures is a direct multidimensional extension of the Chakravarty index from 1983. Like the previous class, this class of poverty measures is also IT efficient; the IT measure for $\delta \neq 0$ as specified in (2) is a version of this class of poverty measures in case $\gamma=\delta$ and $v_{i j}^{\delta}=1-\left(x_{i j} / z_{j}\right)^{\theta}$.

$$
P_{C_{1}}=1 / n \sum_{i \in S_{j}} \sum_{j \in\{1, \ldots, k\}: c_{i j}=1} a_{j}\left(1-\left(x_{i j} / z_{j}\right)^{\theta}\right)
$$

with $a_{j}>0 ; \sum_{j=1}^{k} a_{j}=1 ; \theta \in(0,1)$

This class of indices satisfies is comparable to the previous one, except for the fact that the progression of the function $f(\cdot)$ in this case is less regular in the sense that it is rather steep for very small values of $x_{i j}$ and almost linear afterwards.

\section{The multidimensional Watts class of poverty measures}

This class of poverty measures is a direct multidimensional extension of the Watts index from 1968.

$$
P_{W}=1 / n \sum_{i \in S_{j}} \sum_{j \in\{1, \ldots, k\}, c_{i j}=1} a_{j} \log \left(z_{j} / x_{i j}\right)
$$

with $a_{j}>0 ; \sum_{j=1}^{k} a_{j}=1$

A disadvantage of this class of poverty measures is that the degree of inequality aversion cannot be chosen, as it is simply the logarithm. Another disadvantage is that the measure is unbounded, i.e. its upper bound depends on the units chosen for the poverty thresholds $z_{j}$, and not defined for $x_{i j}=0$. It is, however, IT-efficient; the IT measure for $\delta \neq 0$ as specified in (2) is a normalized version of this class of indices in case $\gamma=\delta$ and $v_{i j}^{\delta}=\log \left(z_{j} / x_{i j}\right)$.

\section{The second multidimensional Chakravarty class of poverty measures}

This class of poverty measures is a non-additive multidimensional extension of the Chakravarty index from 1983, and has been introduced by Tsui (2002).

$$
P_{C_{2}}=1 / n \sum_{i \in S_{j}}\left[\prod_{j \in\{1, \ldots, k\} c_{i j}=1}\left(z_{j} / x_{i j}\right)^{r_{j}}-1\right]
$$

with $r_{j} \in[0,1]$.

This class of poverty measures is no longer additive. Like the former class this class too is unbounded, i.e. its upper bound depends on the units chosen for $z_{j}$. Like the other indices, it is IT-efficient, precisely the IT measure for $\delta=0$ (3) is a normalized version of this class of indices in case $\gamma=1$ and $v_{i j}^{w_{j}}=\left(z_{j} / x_{i j}\right)^{r_{j}}-1$ (Maasoumi and Lugo, 2008, p. 9). 
Bourguignon and Chakravarty (2003) criticised this class of poverty measures for restricting attributes to substitutes. In response, they introduced the following class of poverty measures:

\section{The multidimensional Bourguignon-Chakravarty class of poverty measures}

$$
P_{B C}=1 / n \sum_{i \in S_{j}}\left[\sum_{j \in\{1, \ldots, k\} c_{i j}=1} a_{j}\left(1-x_{i j} / z_{j}\right)^{\theta}\right]^{\beta / \theta}
$$

with $a_{j}>0 ; \sum_{j=1}^{k} a_{j}=1 ; \quad \theta>1 ; \beta \geq \theta \vee \beta \leq \theta$

As Chakravarty and Silber (2008) point out, this class of indices is less simple than Tsui's multidimensional extension since constant elasticity i) is defined between shortfalls rather than attributes, and ii) does not necessarily equal one. However, the most significant difference is that this class does not require attributes to be substitutes but instead allows them to be either substitutes $(\beta>\theta)$ or complements $(\beta<\theta)$. This class of indices resembles the class of IT measures for $\delta \neq 0$ with $v_{i j}=1-x_{i j} / z_{j}, \gamma=\beta$ and $\delta=\theta$.

Please note that the new identification method can be applied to all poverty measures. In case of the additive indices the new identification method ensures fulfilment of IS. All nonadditive poverty measures satisfy IS anyway, however, in case of the multidimensional Bourguignon-Chakravarty class of poverty measures, no solution has been suggested so far that would also ensure the fulfilment of NIPA. Due to considerations with regard to the fulfilment of normalization, factor decomposability and the more regular progression of the function, the remainder of the chapter will focus on the FGT functional form of $f(\cdot)$, introducing the following new class of poverty indices:

\section{The multidimensional Inequality-Sensitive class of poverty measures}

$$
P_{I S}=1 / n \sum_{i \in S_{j}} \delta_{i}^{\alpha} \sum_{j \in\{1, \ldots, k\} c_{c_{j}}=1} a_{j}\left(1-x_{i j} / z_{j}\right)^{\theta}
$$

with $a_{j}>0 ; \sum_{j=1}^{k} a_{j}=1 ; \theta>1 ; 0 \leq \alpha \leq 1$ in case attributes are complements and $\alpha \geq 1$ in case attributes are substitutes.

Table 2.01 compares the different classes of poverty measures according to the properties that they do or do not satisfy.

\begin{tabular}{|c|c|c|c|c|c|c|}
\hline Axioms & $P_{F G T}$ & $P_{C 1}$ & $P_{W}$ & $P_{C 2}$ & $P_{B C}$ & $P_{I S}$ \\
\hline Anonymity (AN) & $\checkmark$ & $\checkmark$ & $\checkmark$ & $\checkmark$ & $\checkmark$ & $\sqrt{L}$ \\
\hline Continuity (CN) & $\checkmark$ & $\checkmark$ & $\checkmark$ & $\checkmark$ & $\checkmark$ & $\checkmark$ \\
\hline Monotonicity (MN) & $\checkmark$ & $\checkmark$ & $\checkmark$ & $\checkmark$ & $\checkmark$ & $\checkmark$ \\
\hline Principle of Population (PP) & $\checkmark$ & $\checkmark$ & $\checkmark$ & $\checkmark$ & $\checkmark$ & $\checkmark$ \\
\hline
\end{tabular}

Table 2.01 Axiomatic Foundation of Selected Cardinal Poverty Measures 
Strong Focus (SF)

Subgroup Decomposability (SD)

Factor Decomposability (FD)

Normalization (NM)

Scale Invariance (SI)

Uniform Majorization (UM)

Nondecreasingness under Association Increasing Switch (NDA)

Nonincreasingness under Association Increasing Switch (NIA)

Nonincreasingness under Pareto-efficient Association Increasing Switch (NIPA)

Inequality Sensitivity (IS)

${ }^{15}$ Only satisfied in case attributes are substitutes, i.e. for $\delta>\alpha$

${ }^{16}$ Only satisfied in case attributes are complements, i.e. for $\delta<\alpha$

In order to analyse the effects of within and between dimensional inequality on poverty measurement, I will utilise the following representative of Alkire and Foster's $M_{0}$ class of indices as a base case:

$$
M_{0}=1 / n \sum_{\substack { i \in S_{j} \\
\begin{subarray}{c}{j \in\{1, \ldots, k\} ; c_{i j} \\
\wedge \delta_{i} \geq \delta_{I M}^{\min }{ i \in S _ { j } \\
\begin{subarray} { c } { j \in \{ 1 , \ldots , k \} ; c _ { i j } \\
\wedge \delta _ { i } \geq \delta _ { I M } ^ { \operatorname { m i n } } } }\end{subarray}} a_{j}
$$

To this index, I will compare the multidimensional FGT class of poverty measures, i.e.:

$$
P_{F G T}(\mathbf{X} ; \mathbf{z})=1 / n \sum_{i \in S_{j}} \sum_{j \in\{1, \ldots, k\}, c_{i j}=1} a_{j}\left(1-x_{i j} / z_{j}\right)^{\theta}
$$

As well as the new multidimensional Inequality-Sensitive class of poverty measures, i.e.:

$$
P_{I S}(\mathbf{X} ; \mathbf{z})=1 / n \sum_{i \in S_{j}} \delta_{i}^{\alpha} \sum_{j \in\{1, \ldots, k\} ; c_{i j}=1} a_{j}\left(1-x_{i j} / z_{j}\right)^{\theta}
$$

However, before turning to the empirical application, I will decompose the two latter indices according to the three poverty components incidence, intensity and inequality ${ }^{22}$.

\section{The Decomposition of the multidimensional FGT class of poverty measures}

The following draws on a decomposition done by Aristondo, Lasso de la Vega and Urrutia (2010) for the one-dimensional case.

\section{Proposition 2.}

$$
P_{F G T}(\mathbf{X} ; \mathbf{z})=H \sum_{j \in\{1, \ldots, k\}: c_{i j}=1} a_{j}\left(q_{j} / q\right)\left\{\left[\mu_{j}(\mathbf{g})\right]^{\theta}\left[1+(\theta(\theta-1)) G E_{\theta}(\mathbf{g})\right]\right\}
$$

With:

i) the headcount ratio, i.e. $H=(q / n)$, measuring the incidence of poverty,

ii) the aggregate poverty gap ratio for attribute $j$, i.e. $\mu_{j}(\mathbf{g})=1 / q_{j} \sum_{i \in S_{j}} g_{i j}$, measuring the intensity of poverty, and

\footnotetext{
${ }^{22}$ Please note that due to its insensitivity with regard to any kind of inequality, $M_{0}$ can only be decomposed into the product of poverty incidence and intensity (Alkire and Santos 2010).
} 
iii) the Generalized Entropy inequality index of the poverty gaps for attribute $j$, i.e. $G E_{\theta}(\mathbf{g})=[1 /(\theta(\theta-1))]\left[1 / q_{j}\right] \sum_{i \in S_{j}}\left\{\left[g_{i j} / \mu_{j}(\mathbf{g})\right]^{\theta}-1\right\}$, capturing within dimensional inequality.

While the multidimensional FGT index does account for inequality within dimensions, it fails to do so for inequality between dimensions. This failure has been justified with the explanation that the index's (wanted) additivity prevents its sensitivity to associationincreasing switches. However, as argued before, association-sensitivity influences inequality between dimensions yet is not the same. The implication of the more holistic approach taken in this chapter becomes obvious once we consider the decomposition of the additive ISPI that comprises both components, within as well as between dimensional inequality.

\section{The Decomposition of the multidimensional Inequality-Sensitive class of poverty} measures

\section{Proposition 3.}

$$
P_{I S}(\mathbf{X} ; \mathbf{z})=H \sum_{j \in\{1, \ldots, k\}: c_{i j}=1} a_{j}\left(q_{j} / q\right)[\mu(\boldsymbol{\delta})]^{\alpha}\left[\mu_{j}(\mathbf{g})\right]^{\theta}\left[1+(\alpha(\alpha-1)) G E_{\alpha}(\boldsymbol{\delta})\right]\left[1+(\theta(\theta-1)) G E_{\theta}(\mathbf{g})\right][I(\mathbf{g}, \boldsymbol{\delta})]
$$

With:

i) the headcount ratio, i.e. $H=(q / n)$, measuring the incidence of poverty,

ii) the aggregate deprivation count ratio, i.e. $\mu(\boldsymbol{\delta})=1 / q \sum_{i \in S_{j}} \delta_{i}$, measuring the intensity of poverty breadth,

iii) the aggregate poverty gap ratio for attribute $j$, i.e. $\mu_{j}(\mathbf{g})=1 / q_{j} \sum_{i \in S_{j}} g_{i j}$, measuring the intensity of poverty depth for attribute $j$,

iv) the GE inequality measure of deprivation counts, i.e. $G E_{\alpha}(\boldsymbol{\delta})=[1 / q(\alpha(\alpha-1))] \sum_{i \in S_{j}}\left[\left[\delta_{i} / \mu(\boldsymbol{\delta})\right]^{\alpha}-1\right], \quad$ measuring inequality between dimensions,

v) the GE inequality measure of poverty gaps for attribute $j$, i.e. $G E_{\theta}(\mathbf{g})=\left[1 / q_{j}(\theta(\theta-1))\right] \sum_{i \in S_{j}}\left[\left[g_{i j} / \mu_{j}(\mathbf{g})\right]^{\theta}-1\right]$, measuring within dimensional inequality for attribute $j$, and, finally,

vi) an interaction term $I(\mathbf{g}, \boldsymbol{\delta})=\left[1 / q_{j} \sum_{i \in S_{j}} \delta_{i} g_{i j}^{\theta} /\left\{\left[1 / q \sum_{i \in S_{j}} \delta_{i}\right]\left[1 / q_{j} \sum_{i \in S_{j}} g_{i j}^{\theta}\right]\right\}\right]$, mapping the interaction between poverty gaps and deprivation counts.

The new class of Inequality-Sensitive poverty measures explicitly accounts for the fact that individuals may suffer from multiple simultaneous deprivations, a fact that is 
axiomatically captured by sensitivity to inequality and enables the most comprehensive decomposition of any additive index developed so far.

\subsection{Empirical Application}

This sub-section illustrates the implications of the new methodology developed in this chapter with data from the Demographic and Health Survey (DHS). As the empirical application is based on a comparison with the inequality insensitive $M_{0}$ as base case it follows many of the choices of its most prominent representative, the Multidimensional Poverty Index (MPI) (Alkire and Santos 2010). Like the choice of the DHS data, nationally representative surveys that are mainly funded by the US Agency for International Development (USAID) and that Alkire and Santos (2010) privilege over other internationally comparable surveys. The final country sample consists of 28 countries for which more or less recent DHS surveys exist and that do not lack any of the indicators chosen for the poverty calculations.

In order to be able to apply cardinal poverty indices, a reasonably meaningful cardinal interpretation of attributes needs to be ensured though these kinds of choices are, admittedly, always problematic and disputable.

The index that I will use for the empirical application is a representative of the new Inequality-Sensitive class of poverty measures called Inequality Sensitive Poverty Index (ISPI). The ISPI consists of the following five equally weighted indicators: maternal health, child health, education, living conditions and asset endowment.

A household is deprived in maternal health if any woman in reproductive age (15-49) has a Body Mass Index (BMI) smaller than 18.5, and in child health if any child has a weight-forage z-score below -2.5 according to World Health Organization (WHO) statistics. These two indicators differ from the rest of the indicators in the sense that they lack definite lower boundaries. Thus, appropriate boundaries are chosen on the basis of medical reports. In the case of the BMI, encyclopedia.com states that " $a$ BMI between 13 and 15 corresponds to 48 to 55 percent of desirable body weight for a given height and describes the lowest body weight that can sustain life". In the case of weight-for-age z-scores, medical research of Bern et al. (1997) revealed that weight-for-age z-scores below -4.4 were no longer associated with an increased risk of mortality. In response, the minimum levels of 14 and -4.5 were chosen for the normalisation of BMI and z-scores, respectively. For all other indicators, the minimum level utilised for normalisation is the natural boundary zero. 
A household is deprived in education if none of its members has at least five years of schooling.

In order to capture the living conditions of a household, I follow a methodology suggested by Bérenger and Bresson (2010) and derive a Composite Living Index (CLI) that comprises quantitative and qualitative aspects of living conditions. Precisely, the number of sleeping rooms per head adjusted by household composition (children under five years of age receive a weight of 0.5 ) is utilised as an indicator for overcrowding. This figure is afterwards refined through the multiplication with a coefficient of penalty that addresses i) structural quality as indicated by flooring conditions and connection for power supply, and ii) the quality of physical amenities as indicated by the quality of drinking water, sanitation, and cooking fuel. For each of these equally weighted indicators, the threshold is the respective MDG standard as used for the calculation of the MPI (compare chapter one, p. 28). Thus, the CLI is calculated as:

$$
\text { CLI }=\operatorname{rooms}(\operatorname{adj} .) *(1-0.1 *(\text { floor }+ \text { electricity }+ \text { water }+ \text { sanitation }+ \text { fuel }))
$$

Following Bérenger and Bresson (2010), I choose 0.3 as threshold for the CLI.

Finally, a Weighted Asset Index (WAI) captures household deprivation in asset endowments. The WAI is the sum of the prevalence-weighted ${ }^{23}$ MPI items, i.e. i) television $(0.15)$, ii) bicycle $(0.16)$, iii) radio $(0.10)$, iv) telephone $(0.18)$, v) motorbike $(0.21)$, and vi) refrigerator (0.20). According to the characteristics of the distribution, households with a WAI below 0.27 that do not own a car or truck are considered deprived.

Figure 2.03 provides an overview of the different dimensions, indicators and thresholds used for the calculation of the ISPI:

Figure 2.03 The Structure of the ISPI

\begin{tabular}{llll}
\hline Dimension & Main Capability & Indicator & Threshold (Household Level) \\
\hline \multirow{2}{*}{ Health } & Bodily Health & Maternal Health & At least one woman age 15-49 with BMI $<18.5$ \\
\cline { 2 - 4 } Education & $\begin{array}{l}\text { Senses, Imagination } \\
\text { and Thought }\end{array}$ & Schooling & No member with at least five years of schooling \\
\hline \multirow{2}{*}{$\begin{array}{l}\text { Living } \\
\text { Standards }\end{array}$} & Bodily Health & Housing & Composite Living Index $(\mathrm{CLI})<0.3$ \\
\cline { 2 - 4 } & $\begin{array}{l}\text { Control over } \\
\text { Environment }\end{array}$ & Assets & $\begin{array}{l}\text { Weighted Asset Index }<0.27 \text { and does not own car or } \\
\text { truck }\end{array}$ \\
\hline
\end{tabular}

$M_{0}$ is calculated with a dual cut-off of $20 \%$ of the weighted sum of indicators. The multidimensional FGT class of indices and the ISPI are calculated for the cases $\theta=\alpha=1.5$ and $\theta=\alpha=2$.

\footnotetext{
${ }^{23}$ Brackets contain the prevalence weights of the respective items. Prevalence weights means that all weights are calculated as the inverse of the frequency with which these items are observed across the sample.
} 


\section{[Table 2.02]}

It is immediately obvious from table 2.02 that distinct rank changes are caused by utilising cardinal indices instead of the ordinal $M_{0}$. Sixteen countries experience rank changes once the multidimensional FGT class of indices is applied instead of $M_{0}$, the highest change being a loss of seven places in the case of Liberia, which is actually huge given the relatively small sample size. As is obvious from the table, this change is mainly due to the high levels of poverty intensity within the two dimensions years of schooling and assets that only cardinal indices are able to capture. Interestingly, Liberia experiences yet another distinct rank change in case the ISPI is utilised instead of the FGT class of indices. Intuitively, since poverty in Liberia is mainly concentrated in two dimensions, inequality between dimensions can be expected to be relatively low, reflected in a lower ISPI value. This is indeed the case. Liberia reduces a lot of the losses induced by its within-dimensional failures in the dimensions education and assets and gains five places back in the ranking once the ISPI is utilised instead of the FGT class of indices.

India, on the other hand, has a rather low degree of inequality within dimensions so that it gains four places in the ranking once the FGT class of indices is utilised in place of $M_{0}$. However, poverty intensity and inequality between dimensions, though not high, are nevertheless distinct, reducing the places gained to two once the ISPI is utilised in place of the FGT class of indices.

Yet another interesting case is Nigeria. Nigeria demonstrates a combination of slightly increased within and between dimensional inequality when compared to its reference countries in the ranking. This characteristic induces a loss of two places once the FGT class of indices is applied instead of $M_{0}$ and a loss of yet another two places once the ISPI is applied instead of the FGT class of indices.

These examples plainly illustrate that the characteristics of poverty in a specific country are more and more uncovered through the change from $M_{0}$ to the FGT class of indices to the ISPI. The importance that is attributed to these characteristics depends of course on the individual choices of $\theta$ and $\alpha$, the parameters that express the aversion against within and between dimensional inequality.

[Table 2.03]

Table 2.03 summarizes the results for the case that parameter values are increased from $\theta=\alpha=1.5$ to $\theta=\alpha=2$, indicating increased levels of inequality aversion. The resulting changes affect especially those countries that either show rather low or rather high levels of 
inequality, as the significance of outliers gets more pronounced as the level of inequalityaversion increases. Nigeria, for instances, looses two additional places in the ranking, one place is lost through the change from $M_{0}$ to the FGT class of indices, the other through the change from the FGT class of indices to the ISPI.

The empirical results reveal the importance of accounting for within and between dimensional inequality: The character of poverty is very different from country to country and the more comprehensively a poverty measure accounts for this, the more accurate is the insight gained into the very character of poverty in a region, country, district etc. This additional insight bears the potential to increase precision and effectiveness of poverty reducing strategies.

\subsection{Conclusions}

In the case of cardinal poverty indices, inequality between dimensions is usually treated as association-sensitivity. However, such an equation seems to be too narrow and has some serious implications on the axiomatic foundation of multidimensional poverty indices. The definition of association-increasing switches as defined so far concentrates solely on efficiency, i.e. whether the attributes that are switched are substitutes or complements. It neglects the issue of who the beneficiary of the respective switch is and how poverty indices might or might not change with a switch of beneficiaries.

In fact, in case the respective attributes are complements, association-increasing switches as they are defined today violate the economic principle of pareto-efficiency. This chapter introduces an additional axiom that ensures pareto-efficiency of association-increasing switches.

But the issue goes even further; in fact it comprises the broader question what happens in case of switches between individuals that are deprived in a different number of dimensions. It is a highly relevant question that is a direct consequence of the restrictive interpretation of inequality between dimensions and in fact reveals that inequality is more than associationsensitivity. More precisely, this chapter follows the approach of the first in defining inequality between dimensions as the association-sensitive spread of simultaneous deprivations across a society. In consequence, the chapter suggests the introduction of a switch between individuals that are deprived in a different number of dimensions whose effect on poverty does not only depend on the relationship among attributes but also on the choice of the beneficiary of the respective switch. It has been demonstrated how the new axiom can be utilised to derive a 
whole new class of cardinal poverty indices. This class is unique in the sense that it is the first cardinal class of additive poverty indices that i) explicitly accounts for inequality between dimensions as the association-sensitive spread of simultaneous deprivations across society, and, as a result, ii) improves the precision and detailedness of poverty profiles, thereby enhancing the targeting of poverty reduction policies.

Though this chapter can only be a first step towards the measurement of inequality between dimensions in a broader sense, the empirical application already provides enough evidence for its relevance and the need for further research in this important area. 


\section{Chapter 3}

\section{Operationalising the Capability Approach The German Correlation Sensitive Poverty Index}

The official measure to analyse poverty in Germany is the at-risk-of-poverty rate, defined as 60 per cent of the median net equivalence income. However, the concept of poverty that is used as the conceptual framework for the national 'Poverty and Wealth' Reports is Sen's capability approach. This chapter provides the first operationalisation of the capability approach in Germany. The rich data source of the German Socio-Economic Panel is utilized in order to introduce a multidimensional poverty index for Germany that is based on the capability approach, or to be exact, on the mathematical formula of the multidimensional Correlation-Sensitive class of poverty measures introduced in the first chapter of this volume. In addition, a multidimensional happiness index is introduced, a concept that is enjoying increasing popularity. All three indices are compared across dimensions, regions and over time, and the results seem to indicate one thing above all: the high added value that is created through poverty indices that complement the traditional income-based at-risk-of-poverty rate.

\subsection{Introduction}

It is not so long ago that the existence of poverty in the social welfare state Germany was officially denied. This became particularly evident during the aftermath of the 1995 World Summit for Social Development in Copenhagen. The Copenhagen Declaration explicitly acknowledges that 'profound social problems, especially poverty, unemployment and social exclusion [...] affect every country' (Copenhagen Declaration on Social Development, Introduction, point 2) and calls upon ratifiers to implement national poverty reports. Yet the German government revealed considerable reluctance to meet the commitment (Kemming and Borbach, 2003, p. 3):

'But while poverty enjoys a top ranking in the 90 s scientific debate, the existence of poverty in Germany was denied by a Federal Government pointing out to a well-functioning social 
security system. That is in line with the fact that the Federal Government believed there was no need for a national report on poverty.'

When the first 'Poverty and Wealth Report' was finally published on 25 April 2001, the German government ventured into uncharted territory. When it did, it relied on the guidance of the income-based at-the-risk-of-poverty rate (AROPR $)^{24}$ that defines well-being in terms of opulence. Besides its advantages, this index suffers from the same weaknesses as every income measure, i.e. in particular the following two restrictive assumptions: i) the existence of perfect and complete markets that is unable to properly account for public goods provision for instance in the area of health and education; and ii) equality of individual conversion factors that disregards the diversity in social and physical environments as well as the whole range of personal heterogeneities that influence the ability of individuals to convert economic resources into whatever they may need (Sen, 2009, p. 255).

At the same time, the German government followed the recommendations of a German research team (Volkert et al., 2004) and decided to use Sen's capability approach (Sen, 1979; $1985 ; 1992 ; 1999 ; 2009)$ as the conceptual framework for the national 'Poverty and Wealth Reports'. That decision was endorsed after the 2005 elections by the new government (Arndt and Volkert, 2007). The Capability Approach has a very intriguing way to define poverty, especially in the context of affluent countries. By making capabilities and functionings, i.e. what a person is actually able to do and be, the subject of analyses rather than economic resources, it can essentially dispense with the two assumptions of perfect and complete markets and equal individual conversion factors. Then, by utilising a concept of equality of opportunity, poverty is defined as a restricted set of essential capabilities that are needed to pursue whatever one has reason to value.

It is the respect for the freedom and responsibility of the individual that makes this approach so attractive, especially in the context of affluent countries. Income disparities are bad if and only if they are caused by inequality of opportunity, i.e. in case access to such essential institutions as the education system, the labour market and health care are restricted on grounds of gender, origin or anything else of a discriminatory nature, irrespective of

\footnotetext{
${ }^{24}$ The rate is defined as the percentage of the population with a net equivalence income below $60 \%$ of the median. The concept of the net equivalence income accounts for the fact that bigger households have saving opportunities through the joint use of household items. Therefore, the new OECD scale attributes a weight of one to the first adult, a weight of 0.5 for every additional person aged 15 or over and a weight of 0.3 for persons below the age of 15 . Thus, the net equivalence income is the household's net income divided by the weighted sum of household members.
} 
whether the individual concerned lives in a poor or affluent country ${ }^{25}$. On the other hand, inequality that is caused by free individual choice might even be desirable: Rewarding investments attracts additional investments that expand the overall capability set of a society which is, obviously, to everyone's benefit (see Marrero and Rodgriguéz, 2010). ${ }^{26}$

This chapter takes a first step towards the operationalisation of the official German poverty definition by proposing a multidimensional poverty index that is based on the capability approach, the so called German Correlation Sensitive Poverty Index (GCSPI). In addition, it introduces a subjective poverty index, the so called Subjective Correlation Sensitive Poverty Index (SCSPI), a representative of traditional welfarism theory that defines well-being in terms of happiness. ${ }^{27}$

Afterward, a thorough poverty analysis is conducted for the period 2002 to 2010, including dimensional and regional decompositions, in order to compare the three indices that represent the main theories of welfare economics. The empirical analysis provides evidence how the different indices might arrive at entirely different assessments of poverty and poverty trends in Germany, a fact that reveals the high additional value that is generated in case the traditional income-based AROPR is complemented by other, multidimensional poverty indices.

First, there is a strong discrepancy in the identification of the poor according to the AROPR and the GCSPI. In fact, the overlap between the AROPR and the different nonincome GCSPI dimensions is always less than $50 \%$ and in one case even less than $10 \%$. In addition, there is a non-negligible amount of individuals that are not deprived in any of the six non-income dimensions of the GCSPI and yet income-poor.

Second, there exists a considerably discrepancy in the regional composition of the three indices. With only few exceptions, the SCSPI is highest in the Western Bundeslaender, followed by the GCSPI and, finally the AROPR. In the Eastern Bundeslaender, the trend is just the opposite, i.e. the AROPR is highest, followed by the GCSPI and, finally, the SCSPI.

\footnotetext{
${ }^{25}$ The fact that the children of uneducated families are far less likely to make it into grammar schools than the children of the educated middle class shows that these barriers also exist in Germany (Education in Germany, 2012)

${ }^{26}$ These considerations receive strong evidentiary support by a recent study of Marrero and Rodgriguéz (2010) who demonstrate that no robust relationship can be established between overall inequality and growth. However, there exists a robust and significant positive relationship between inequality of choice and economic growth and a robust significant negative relationship between inequality of opportunity and economic growth.

${ }^{27}$ The latter has recently experienced a rather sudden resurrection in the empirical analysis of poverty despite the fact that the related theory has suffered tremendous setbacks in the nineteen-thirties due to the problems connected with the comparison of individual happiness (Robbins, 1938, p. 640; Sen, 2009, p.277-278).
} 
Third, the three indices do not only demonstrate rather different degrees of volatility over time, poverty dynamics can even be opposite. The SCSPI is rather volatile and tends to be higher in more populous, urbanised areas. The AROPR, on the other hand, is less volatile and tends to be higher in less populous, rural areas. By definition it is rather sensitive to the overall inequality in the society and, as a consequence, might decrease during a time in which the situation of the poor actually worsened, i.e. whenever the situation of the poor worsens less than the situation of the wealthy. The GCSPI, finally, is unable to reflect these changes in the overall inequality of the society; it only captures inequality changes among the poor. As a consequence, the poverty trends indicated by these two indices diverge in times of economic crisis in which, generally speaking, all population parts suffer but in which, due to the German social protection system, the situation of the poor usually worsens less than the situation of the better-off.

In summary, the results all seem to point in one direction, namely that the introduction of additional multidimensional poverty indices that complement the AROPR seems to be very worthwhile as these indices provide information about poverty that traditional income-based poverty measures are unable to discover.

The chapter proceeds as follows. The second section provides a brief introduction in the theoretical background, followed by a brief overview in section three of the axiomatic foundation of the multidimensional class of indices that is utilised for the multidimensional indices GCSPI and SCSPI. The fourth section is dedicated to the thorough development of the GCSPI, in particular the choice of dimensions, indicators, thresholds and weights. The empirical application is presented in section five, section six concludes.

\subsection{Theoretical Background}

Let $\mathbb{R}^{\mathrm{k}}$ denote the Euclidean $k$-space, and $\mathbb{R}_{+}^{\mathrm{k}} \subset \mathbb{R}^{\mathrm{k}}$ the non-negative $k$-space. Further, let $\mathbb{N}$ denote the set of positive integers. $\mathbf{N}=\{1, \ldots, n\} \subset \mathbb{N}$ represents the set of $n$ individuals of a typical society and $\mathbf{D}=\{2, \ldots, d\} \subset \mathbb{N}$ the set of $d$ poverty dimensions captured by a set of $k$ poverty attributes $\mathbf{K}=\{2, \ldots, k\} \subset \mathbb{N}$. Let $\mathbf{a} \in \mathbb{R}_{+}^{\mathbf{K}}$ denote the weight vector for the different attributes with $\sum_{j=1}^{k} a_{j}=1$.

In the following, I will refer to the quantity of an attribute with which an individual is endowed as an achievement. The achievement vector of individual $i$ is represented by $\mathbf{x}_{i}=\left(x_{i 1}, \ldots, x_{i k}\right)$ and the respective achievement matrix of a society with $n$ individuals by $\mathbf{X} \in \mathbb{R}_{+}^{\mathrm{NK}}$ where the $i j$ th entry represents the achievement $x_{i j}$ of individual $i$ in attribute $j$. Let 
$X_{\mathrm{n}}$ be the set of possible achievement matrices of population size $n$ and $X=\mathrm{U}_{\mathrm{N}} \subset_{\mathbb{N}} X_{\mathrm{n}}$ the set of all possible achievement matrices. Let $z_{j}$ denote the poverty threshold of attribute $j$ so that individual $i$ is deprived in $j$ whenever the respective achievement falls short of the threshold level, i.e. whenever $x_{i j}<z_{j}$. Further, let $\mathbf{z} \in \mathbb{R}_{++}^{\mathbf{K}}$ represent the vector of poverty thresholds chosen for the different attributes, with the $j$ th element being $z_{j}$, and $\mathbf{Z}$ being the set of all possible vectors of poverty thresholds.

In the context of this chapter, a poverty index is a function $P: X \times \mathbf{Z} \rightarrow \mathbb{R}$. For any poverty threshold vector $\mathbf{z} \in \mathbf{Z}$, society $\mathcal{A}$ has a higher poverty level than society $\mathscr{B}$ if and only if $P\left(\mathbf{X}^{\mathcal{A}} ; \mathbf{z}\right) \geq P\left(\mathbf{X}^{\mathcal{B}} ; \mathbf{z}\right)$ for any $\mathbf{X}^{\mathcal{A}}, \mathbf{X}^{\mathscr{B}} \in X$.

Let $\mathbf{c}_{i}=\left(c_{i 1}, \ldots, c_{i k}\right)$ represent the deprivation vector of individual $i$ such that $c_{i j}=1$ if $x_{i j}<z_{j}$ and $c_{i j}=0$ if $x_{i j} \geq z_{j}$. Further, let $S_{j}(\mathbf{X})$ - or simply $S_{j}$ - denote the set of individuals who are poor with respect to attribute $j$, and $q$ the overall number of poor individuals in a society.

For reasons of simplicity, let $\delta_{i}=\sum_{j \in\{1, \ldots, k\} c_{c_{j}}=1} a_{j}$ denote the sum of weighted deprivations suffered by individual $i$.

However, in a multidimensional framework it does not suffice to determine those who are deprived. In addition, it has to be defined how deprived a person has to be in order to be considered poor. This is the task of the identification function $\rho: \mathbb{R}_{+}^{\mathbf{K}} \times \mathbb{R}_{++}^{\mathbf{K}} \rightarrow\{0,1\}$ so that individual $i$ is poor if $\rho\left(\mathbf{c}_{i} ; \mathbf{z}\right)=1$ and not poor if $\rho\left(\mathbf{c}_{i} ; \mathbf{z}\right)=0$.

As has been demonstrated in the previous two chapters, there is only one identification function so far that considers the relationship between inequality and correlation-sensitivity. ${ }^{28}$ It is a multi-step function of the following type:

$$
\rho_{c s}\left(\mathbf{c}_{i} ; \mathbf{z}\right)=\left\{\begin{array}{clc}
\delta_{i}^{\alpha} & \text { if } & \max \left\{\mathbf{c}_{i \cdot}\right\}=1 \\
0 & \text { if } & \max \left\{\mathbf{c}_{i \cdot}\right\}=0
\end{array}\right.
$$

$\rho_{c s}\left(\mathbf{c}_{i} ; \mathbf{z}\right)$ is in a way a fuzzy approach that differentiates between the poor on one hand and different degrees of poverty severity on the other. The specific shape of the function is of intriguing simplicity: the function is always non-decreasing in the number of deprivations, however, the marginal increase in poverty severity is the less the higher the substitutability between attributes. Whereas the former accounts for inequality, the latter ensures correlationsensitivity. The relationship between the two is determined by alpha.

\footnotetext{
${ }^{28}$ Usually, the two concepts are equated rendering a sound distinction between the two concepts of distributive justice and efficiency impossible, despite the fact that such a distinction is indispensable for sound poverty measurement.
} 
As has been demonstrated in the previous two chapters, this inequality- and correlationsensitive way to identify the poor allows composite indices to satisfy two core properties that were hitherto considered to be impossible to be fulfilled at the same time. The first is Factor Decomposability (FD) that allows the decomposition of the index according to poverty dimensions and indicators. The second is Sensitivity to Inequality Increasing Switch (SIIS) in the case of ordinal poverty indices, and Inequality Sensitivity (IS) in the case of cardinal indices. The latter properties ensure the sensitivity of the final indices to both distributive justice as well as efficiency considerations.

As the purpose of this chapter is to make a first proposal for a multidimensional poverty index for Germany and to study its strengths and weaknesses, I decided to concentrate on the simpler ordinal approach, i.e. to use the mathematical formula of the Correlation Sensitive Poverty Index (CSPI), i.e. formula 1.10. However, as suggested in the previous chapter, the clear distinction between the concepts of distributive justice and efficiency can as well be introduced in the case of cardinal poverty indices. The resulting Inequality Sensitive Poverty Index (ISPI) could be utilised in the German context in the same way as the Correlation Sensitive Poverty Index (CSPI) suggested in this chapter - of course only for those data that permit a cardinal approach.

\subsection{The Axiomatic Foundation and Decomposition}

The axiomatic approach provides the most transparent way to derive a poverty index by explicitly defining properties that it may or may not satisfy (Deutsch and Silber, 2005). This is the reason why the CSPI has been derived axiomatically. As the latter has already been discussed extensively in the first chapter, this section will only provide a brief overview of the core axioms that the index satisfies (e.g. Chakravarty, Mukherjee and Ranade, 1998; Bourguignon and Chakravarty, 1999; Tsui, 2002; Bourguignon and Chakravarty, 2003; Chakravarty and Silber, 2008).

Anonymity (AN): For any $\mathbf{z} \in Z$ and $\mathbf{X} \in X_{\mathrm{n}}, P(\mathbf{X} ; \mathbf{z})=P(\boldsymbol{\Pi X} ; \mathbf{z})$ where $\boldsymbol{\Pi}$ is any permutation matrix of appropriate order.

Continuity (CN): For any $\mathbf{z} \in Z$ and $\mathbf{X} \in X_{\mathrm{n}}, P(\mathbf{X} ; \mathbf{z})$ is continuous on $\mathbb{R}_{+}^{\mathrm{NK}}$.

Monotonicity (MN): For any $\mathbf{z} \in Z$ and $\mathbf{X}, \mathbf{X}^{\prime} \in X_{\mathrm{n}}$, if for any individual $h$ and any attribute $l$ $x_{h l}=x_{h l}^{\prime}+\beta, \quad$ such $\quad$ that $\quad x_{h l}^{\prime}<z_{l}, \beta>0$, and $\quad x_{i l}=x_{i l}^{\prime} \forall i \neq h, \quad x_{i j}=x_{i j}^{\prime} \forall j \neq l, \forall i$, then $P\left(\mathbf{X}^{\prime} ; \mathbf{z}\right) \leq P(\mathbf{X} ; \mathbf{z})$. 
Principle of Population (PP): If for any $\mathbf{z} \in Z, \mathbf{X} \in X_{\mathrm{n}}$, and $m \in \mathbb{N} \mathbf{X}^{m}$ is a $m$-fold replication of $\mathbf{X}$, then $P\left(\mathbf{X}^{m} ; \mathbf{z}\right)=P(\mathbf{X} ; \mathbf{z})$.

Strong Focus (SF): For any $\mathbf{z} \in Z$ and $\mathbf{X} \in X_{\mathrm{n}}$, if for any individual $h$ and any attribute $l$ $x_{h l} \geq z_{l}, x_{h l}^{\prime}=x_{h l}+\beta, \beta>0$, and $x_{i l}^{\prime}=x_{i l} \forall i \neq h, x_{i j}^{\prime}=x_{i j} \forall j \neq l, \forall i$, then $P(\mathbf{X} ; \mathbf{z})=P\left(\mathbf{X}^{\prime} ; \mathbf{z}\right)$.

Subgroup Decomposability (SD): For any $\mathbf{X}^{1}, \ldots, \mathbf{X}^{v} \in X_{\mathrm{n}}$ and $\mathbf{z} \in Z$, $P\left(\mathbf{X}^{1}, \mathbf{X}^{2}, \ldots, \mathbf{X}^{v} ; \mathbf{z}\right)=\sum_{l=1}^{v} n_{l} / n P\left(\mathbf{X}^{l} ; \mathbf{z}\right)$ with $n_{l}$ being the population size of subgroup $\mathbf{X}^{l}, l=1, \ldots, v$ and $\sum_{l=1}^{v} n_{l}=n$.

Factor Decomposability (FD): For any $\mathbf{z} \in Z$ and $\mathbf{X} \in X_{\mathrm{n}}, P(\mathbf{X} ; \mathbf{z})=\sum_{j=1}^{k} a_{j} P\left(x_{\cdot j} ; z_{j}\right)$

Normalization (NM): For any $\mathbf{z} \in Z$ and $\mathbf{X} \in X_{\mathrm{n}}, P(\mathbf{X} ; \mathbf{z})=1$ if $x_{i j}=0 \forall i, j$ and $P(\mathbf{X} ; \mathbf{z})=0$ if $x_{i j} \geq z_{j} \forall i, j$. Thus, $P(\mathbf{X} ; \mathbf{z}) \in[0,1]$.

Scale Invariance (SI): For any $\mathbf{z} \in Z$ and $\mathbf{X}, \mathbf{X}^{\prime} \in X_{\mathrm{n}}, P(\mathbf{X} ; \mathbf{z})=P\left(\mathbf{X}^{\prime} ; \mathbf{z}^{\prime}\right)$ where $\mathbf{X}^{\prime}=\mathbf{X} \mathbf{\Lambda}$; $\mathbf{z}^{\prime}=\Lambda \mathbf{z}$ with $\boldsymbol{\Lambda}$ being the diagonal matrix $\operatorname{diag}\left(\lambda_{1}, \ldots, \lambda_{k}\right), \lambda_{j}>0 \forall j$.

Sensitivity to Inequality Increasing Switch (SIIS): For any $\mathbf{z} \in Z$ and $\mathbf{X}, \mathbf{X}^{\prime}, \mathbf{X}^{\prime \prime} \in X_{\mathrm{n}}$ if $\mathbf{X}^{\prime}$ is obtained from $\mathbf{X}$ by an inequality increasing switch of non-complementary attributes, then $P(\mathbf{X} ; \mathbf{z}) \leq P\left(\mathbf{X}^{\prime} ; \mathbf{z}\right)$. Further, if $\mathbf{X}^{\prime \prime}$ is obtained from $\mathbf{X}$ by an inequality increasing switch of complement attributes, then $P(\mathbf{X} ; \mathbf{z}) \underset{\leq}{\geq}\left(\mathbf{X}^{\prime \prime} ; \mathbf{z}\right) \leq P\left(\mathbf{X}^{\prime} ; \mathbf{z}\right)$.

AN requires that any personal characteristics apart from the respective achievement levels are irrelevant for poverty measurement. $\mathrm{CN}$ is a rather technical requirement precluding the oversensitivity of poverty measures. MN requires poverty measures not to increase if, ceteris paribus, the condition of a deprived individual improves. PP precludes the dependence of poverty measures from population size and thus allows for cross-population and -time comparisons of poverty. SF demands that giving a person more of an attribute with respect to which this person is not deprived will not change the poverty measure. FD and SD facilitate the calculation of the contribution of different subgroup-attribute combinations to overall poverty, improving the targeting of poverty-alleviating policies. NM is a simple technical property requiring poverty measures to be equal to zero in case all individuals are non-poor and equal to one in case all individuals are poor. SI requires that a proportional distribution should leave inequality levels unchanged, ensuring that poverty indices do not change with the unit of measurement. Finally, SIIS requires that a switch of attributes that increases (reduces) the number of deprivations suffered by the person with higher (lower) initial deprivation will increase poverty in case of a non-complementary relationship between 
attributes. In case attributes are complements, however, it depends on the degree of complementarity as well as the level of importance that is attributed to considerations of distributive justice whether poverty increase or decrease through such a switch.

As has been demonstrated in chapter one, the following family of poverty indices is the only one able to satisfy all aforementioned properties:

$$
P(\mathbf{X} ; \mathbf{z})=1 / n \sum_{i \in S_{j}} f\left(\mathbf{c}_{i \cdot}\right) \sum_{j \in\{1, \ldots, k\}, c_{i j}=1} a_{j}
$$

with $\sum_{j=1}^{k} a_{j}=1$ and $f\left(\mathbf{c}_{i}\right): \mathbb{R}_{+}^{\mathbf{K}} \times \mathbb{R}_{++}^{\mathbf{K}} \rightarrow(0,1]$ non-decreasing in $d_{i}=\#\left\{c_{i j} \mid c_{i j}=1\right\}$ with a nondecreasing (nonincreasing) marginal ${ }^{29}$ in case attributes are considered to be substitutes (complements).

An intuitively appealing index that belongs to this family is the CSPI that is defined as follows:

$$
P_{C S P I}(\mathbf{X} ; \mathbf{z})=1 / n \sum_{i \in S_{j}} \delta_{i}^{\alpha} \sum_{j \in\{1, \ldots, k\}: c_{i j}=1} a_{j}
$$

As demonstrated in chapter one, the CSPI is the only ordinal and additive poverty index that can be decomposed into all three "I's of poverty" (Jenkins and Lambert, 1997, p. 317), i.e. incidence, intensity and inequality:

$$
P_{C S}(\mathbf{X} ; \mathbf{z})=H \cdot \mu(\boldsymbol{\delta})^{\alpha+1} \cdot\left[1+2 G E_{\alpha+1}(\boldsymbol{\delta})\right]
$$

with $\alpha>0$, the headcount ratio $H=(q / n)$ measuring the incidence of poverty, the aggregate deprivation count ratio $\mu(\boldsymbol{\delta})=(1 / q) \sum_{i \in S_{j}} \sum_{j \in\{1, \ldots, k\} c_{i j}=1} a_{j}$ measuring the intensity of poverty breadth and the GE inequality measure of deprivation counts $G E(\boldsymbol{\delta})=\left[1 / q\left(\theta^{2}-\theta\right)\right] \sum_{i \in S_{j}}\left[\delta_{i} / \mu(\boldsymbol{\delta})\right]^{\theta}-1$ capturing the inequality of the distribution of deprivations among the poor.

Please note that poverty incidence as defined in this decomposition is in fact the headcount of all those who are deprived. In other words, it is very high due to the fact that it neglects the differences in the levels of poverty severity that exist between the individuals. However, this differentiation is a key element of the fuzzy approach to poverty measurement as described in the previous section. Thus, in the empirical application, I will separate the overall headcount into the following three categories:

Category one: Deprivation affected. Individuals are classified as deprivation affected whenever the weighted sum of their deprivations is one third or less, i.e. $\sum_{i \in S_{j}} \delta_{i} \leq 1 / 3$. It is

\footnotetext{
${ }^{29}$ A function $f(x)$ has a nondecreasing marginal if $f\left(x_{g}+1\right)-f\left(x_{g}\right) \geq f\left(x_{h}+1\right)-f\left(x_{h}\right)$ whenever $x_{g} \geq x_{h}$.
} 
important from a policy perspective to have them on the radar in order to ensure that their situation does not further deteriorate. However, no action has to be taken at that level. Thus, whenever I will compare different headcounts in the following empirical analysis, I will only concentrate on the headcount of those who belong either to category two or category three.

Category two: Poor. Individuals are classified as poor whenever the weighted sum of deprivations is higher than one third but not higher than two thirds, i.e. $1 / 3<\sum_{i \in S_{j}} \delta_{i} \leq 2 / 3$.

Category three: Severely poor. Individuals are classified as severely poor whenever the weighted sum of their deprivations is higher than two third, i.e. $\sum_{i \in S_{j}} \delta_{i}<2 / 3$. These are the poorest of the poor whose capability set is limited in such a way that it is almost impossible for them pursue their goals in life. At least out of considerations of distributive justice, they are the ones who should be high on the political agenda.

I shall come back to that specific issue if classification in the empirical application.

\subsection{The German Correlation Sensitive Poverty Index}

The following section is devoted to the derivation of the German Correlation Sensitive Poverty Index (GCSPI). The mathematical formula is that of the CSPI for $\alpha=1:^{30}$

$$
C S P I_{\alpha=1}=1 / n \sum_{i \in S_{j}} \delta_{i} \sum_{j \in\{1, \ldots, k\} c_{c i j}=1} a_{j}
$$

In the following, I will utilise the German Socio-Economic Panel (GSOEP) in order to suggest poverty dimensions and indicators specifically for the German context. The GSOEP is a representative longitudinal panel data set collecting socio-economic information at the household level in Germany since 1984. After the German reunification in 1990, the data set has been expanded in order to cover the former German Democratic Republic (DDR). The survey is repeated annually with every adult in a household aged sixteen years or older being surveyed (Wagner, Frick and Schupp, 2007).

I decided to take advantage of the fact that the GSOEP surveys every household member aged sixteen years or older by using this rare opportunity to measure poverty on the individual rather than the household level. Hence, only responses for adults are utilized for the calculations. Also, the existence of missing values in one or more of the chosen indicators was countered with the removal of the whole observation. This treatment led to a considerable

\footnotetext{
${ }^{30}$ This conservative way to choose $\alpha$ suits particularly well as long as a deeper analysis of the relationship between poverty dimensions is lacking. Once more is known about the relationship between poverty dimensions, other levels of $\alpha$ might prove to be more appropriate.
} 
reduction in the final sample size. ${ }^{31}$ Please note that this reduction in the sample size is the reason for the discrepancy between the at-risk-of poverty rates (AROPR) calculated in this chapter and those that are officially reported in the German poverty reports.

Finally, many of the indicators chosen in the following are only available from 2002 onwards and in some cases have been collected only every two years. Thus, I will restrict the empirical analysis to the time period 2002 to 2010, calculating all indexes every two years (i.e. 2002, 2004, 2006, 2008, 2010).

In order to identify the minimum capability set, comprising those central functionings that are necessary in order to pursue whatever one has reason to value, this chapter starts with the theoretical approach of Martha Nussbaum (Nussbaum, 2003). Martha Nussbaum's work is typically considered to be the most influential and thorough operationalisation of the capability approach developed so far. The female philosopher draws heavily on the work of Aristotle in proposing the following list of 'central human capabilities' (Nussbaum 2003, pp. 41-42):

1. Life: 'Being able to live to the end of a human life of normal length; not dying prematurely, or before one's life is so reduced as to be not worth living.'

2. Bodily Health: 'Being able to have good health, including reproductive health; to be adequately nourished; to have adequate shelter.'

3. Bodily Integrity: 'Being able to move freely from place to place; to be secure against violent assault, including sexual assault and domestic violence; having opportunities for sexual satisfaction and for choice in matters of reproduction.'

4. Senses, Imagination and Thought: 'Being able to use the senses, to imagine, think, and reason - and to do these things in a 'truly human' way, a way informed and cultivated by an adequate education, including, but by no means limited to, literacy and basic mathematical and scientific training. Being able to use imagination and thought in connection with experiencing and producing works and events of one's own choice, religious, literary, musical, and so forth. Being able to use one's mind in ways protected by guarantees of freedom of expression with respect to both political and artistic speech, and freedom of religious exercise. Being able to have pleasurable experiences and to avoid nonbeneficial pain.'

5. Emotions: 'Being able to have attachments to things and people outside ourselves; to love those who love and care for us, to grieve at their absence; in general, to love, to

\footnotetext{
${ }^{31}$ Figure 3.02 on p. 81 provides a detailed overview of the missing values for the different indicators of the GCSPI.
} 
grieve, to experience longing, gratitude, and justified anger. Not having one's emotional development blighted by fear and anxiety.'

6. Practical Reason: 'Being able to form a conception of the good and to engage in critical reflection about the planning of one's life.'

7. Affiliation: 'A. Being able to live with and toward others, to recognize and show concern for other human beings, to engage in various forms of social interaction; to be able to imagine the situation of another. [...] B. Having the social bases of self-respect and nonhumiliation; being able to be treated as a dignified being whose worth is equal to that of others. This entails provisions of nondiscrimination on the basis of race, sex, sexual orientation, ethnicity, caste, religion, national origin.'

8. Other Species: 'Being able to live with concern for and in relation to animals, plants, and the world of nature.'

9. Play: 'Being able to laugh, to play, to enjoy recreational activities.'

10. Control Over One's Environment: 'A. Political. Being able to participate effectively in political choices that govern one's life; having the right of political participation, protections of free speech and association. B. Material. Being able to hold property (both land and movable goods), and having property rights on an equal basis with others; having the right to seek employment on an equal basis with others; having the freedom from unwarranted search and seizure. In work, being able to work as a human being, exercising practical reason, and entering into meaningful relationships of mutual recognition with other workers.'

Though such a theoretically derived list can of course never have universal validity and can only serve as a useful guide it nevertheless receives additional legitimacy by the fact that the same list served as a basis for the roundtable discussions of public advisors and scientific experts involved in the development of the German Poverty and Wealth Report (Arndt and Volkert, 2007).

In this context, it is also interesting to take note of the results of a recently conducted survey of German families that questioned respondents about what they conceive to be the most important political tasks in Germany (Monitor Familienleben (i.e. Family Life), 2012). The respondents identified the following four areas: i) to 'fight unemployment' (79\%), related to capability number ten, ii) to 'promote young families with children' (50\%), related to capability number four and seven, iii) to 'reform the health system' (49\%), related to capabilities number one, two and three, and iv) to improve the reconciliation of family and 
work life' $(47 \%)$, related to capabilities number seven and nine and, though to a minor degree, capability number eight.

Interestingly enough, the capabilities that were not covered by the responses are capabilities number five and six, i.e. those capabilities that can hardly be captured by any indicator. The latter is also the reason why these capabilities are not considered in the following drawing up of the GCSPI, a decision that is considerably cushioned by the fact that they have not been mentioned in the survey. That does, however, not apply to capability number one whose operationalisation would be desirable and also seems to be feasible. For instance "life expectancy" would be a good indictor to capture it. Nevertheless this specific capability could not be considered in the drawing up of the GCSPI as the GSOEP does not provide enough information to calculate such an indicator.

Thus, the following drawing up of the GCSPI is based on the capabilities 'bodily health'; 'bodily integrity'; 'senses, imagination and thought'; 'affiliation'; 'other species'; 'play'; and 'control over one's environment'. Whenever possible, the choice of indicators to capture these capabilities is based on the indicators that were proposed during the aforementioned roundtable discussions (Arndt and Volkert, 2007).

However, when it comes to the choice of threshold levels, I deliberately refrain to follow the suggestions. The reason is that the threshold levels proposed during the discussion are rather often relative, typically a percentage of the median value. Despite the fact that such an approach in a composite index with several dimensions would lead to inapplicably high poverty rates - even if categorized as suggested in the previous section - I also want to set a counterexample to the relative nature of indices like the AROPR. Thus, I will utilise the legal minimum requirements as threshold levels whenever possible.

In the following, I will discuss the dimensions, indicators and threshold levels that have been chosen for the drawing up of the GCSPI.

The first dimension chosen for the GCSPI is health and captures mainly capability number two, i.e. 'bodily health'. However, it influences a lot of other capabilities as well. Suffering from bad health limits a person's capability to participate in social life, negatively influences emotions and might even prevent the person to practise his or her occupation. Also, those concerned would typically need more money than their fellow citizens as they are often forced to invest considerable amounts of money in medical treatment. Two indicators are used to capture this dimension, "bad health condition" and "severe health impairments".

Bad health condition is based on the self-evaluation of respondents on a scale ranging from one to five (i.e. 'very good', 'good', 'satisfactory', 'poor', 'bad'). Anyone considering 
his or her health status to be either 'poor' or 'bad' is considered to be deprived according to this indicator. Severe health impairments is also based on a self-evaluation of respondents according to the following five impairments: i) has trouble climbing stairs, ii) health limits vigorous activities, iii) achieved less due to physical health condition, iv) achieved less due to mental health condition, v) reduced social contacts due to health problems. Anyone who reports to suffer from at least four of these impairments is considered to be deprived according to this indicator.

Since I just argued that my intention is to base the GCSPI on absolute and objective criteria instead of always subjective self-evaluations, this exception from my own principle needs some explanation. There has been a lot of discussion recently about the best way to capture individual health conditions that is due to a new research direction in anthropology initiated by Arthur Kleinman and others (Kleinman, Eisenberg, and Good, 1978; Kleinman, 1988; Sen, 2009). The experts strongly criticise the traditional way of utilising health statistics to evaluate health in a society. Their argument is simple but strong: bad health is first and foremost a matter of self-evaluation for if an individual claims to feel bad, i.e. to suffer impairments, to feel pain, etc., who can by any means claim this self-evaluation to be wrong? Thus, Kleinman defines illness as the innately human experiences of symptoms and suffering' (Kleinman, 1988, p. 3) that has to be captured by patient interviews. The questions he proposes for this self-evaluation have become known as 'Kleinman's Questions'. Considering the strength of arguments and the fact that this is the current state-of-the-art approach in anthropology, I decided to use, for once, these subjective indicators.

Arndt and Volkert (2007) suggest yet another indicator. This indicator is "insufficient access to health care" and is based on whether individuals who suffered health impairments within the last three months prior to the interview visited a physician. In case they did not, they are considered to be deprived according to this indicator. I decided not to utilise this indicator for mainly two reasons.

The first reason is that a crucial question is missing in the survey, the question why a person who suffered health impairments did not visit a physician. As pointed out, intention is a crucial aspect in the capability approach: a person should only be considered deprived if he or she would prefer to visit a physician but, for whatever reason, lacks access. Since such a question is missing in the survey, the indicator would involuntarily also consider those persons as deprived who actually have access to a physician but, for whatever reason, prefer not to visit one. 
The second reason is related to the so called "Praxisgebühr", an extra payment of 10 EUR that all patients covered by public health insurance had to make when visiting a physician once in each quarter. This extra payment - which was in effect from 2004-2012 has often been considered one of the main limitations in access to health care and would be a justification for including the suggested "insufficient access" indicator in the calculations of the GCSPI. In order to assess the validity of this statement, I took advantage of the fact that the payment was introduced in 2004. If the payment indeed limited the access to health care, there should be a noticeable difference between the percentage of the population not visiting a physician despite health impairments in 2002 (i.e. before the introduction of the payment) and the subsequent years. However, no such difference can be detected, the respective percentages remain almost constant. This fact is an additional reason why I refrained to include the indicator, though the first reason is the main one.

The second dimension of the GCSPI is education and captures mainly capability number four, i.e. 'senses, imagination and thought', but has a distinct influence on other capabilities as well, such as occupational choice and future income, but also on emotional issues like self-confidence and, connected with this, the ability to engage in social interaction. In fact, there is hardly any other dimension that has such a potential to seriously limit the size of the capability set available in the future. It is then all the more worrying that educational achievements in Germany are strongly correlated with children's social background, introducing rather strong distortions in the objective of a level playing field (Education in Germany, 2012). In addition, the strong influence of the parents on the educational achievements of their children makes income a rather bad indicator: a recent analysis revealed that children with at least one working parent have better chances than children whose parents are unemployed - even if the former have to get along with less money than the latter. This is yet another argument why it might be worthwhile to complement traditional income poverty measures with multidimensional poverty measures.

The dimension education is captured by two indicators. The first one is "school drop out" and is again based on Arndt and Volkert (2007). The deprivation threshold for this indicator, however, is based on compulsory schooling, which in Germany is either nine or ten years of schooling, depending on the respective Bundesland. Thus, any person who dropped out of school with less than nine years of schooling is considered deprived according to this indicator.

The second indicator is "no graduation or training qualification". That indicator captures the aspect that a person might have spent nine years in school, however, without 
graduation. Or, even in case a person was able to complete secondary education, he or she might not have received any further training qualification that is part of the German dual education system and would be needed in order to enter the labour market. Thus, any person who left school without graduation and / or training qualification will be considered deprived according to this indicator.

The third dimension in the GCSPI is employment and directly captures the capabilities number nine and ten, i.e. 'play' and 'control over one's environment'. Since I would otherwise run into a problem with missing values, I decided to capture this dimension by a composite indicator with the following three components: i) "main personal activity status unemployed", ii) "working poor", and iii) "time poor".

With regard to the first component, "main personal activity status unemployed", it is crucial to note that employment is a lot more than just a source of income. Indeed, a recent study analysing poverty in Europe finds 'evidences that income sources and socio-economic endowments, and not only income level, matter for the individual well-being' (Figari, 2012, p. 416). This finding is strongly connected with issues like self-respect, something what Adam Smith described as the ability to appear in public without shame (Smith, 1776: p. 466-67). Though there are of course those who seemingly enjoy the fact that they do not need to work, there are also many persons who would willingly even sacrifice money in order to be able to claim that he or she earned what he or she has. Otherwise the considerable amount of working poor, i.e. those who work for such small income that they depend on additional social security benefits to get along, could never be explained. Thus, I consider those as deprived who are registered as seeking employment but whose main personal activity status over the year has been unemployed.

However, to consider only unemployment within the employment dimension falls way too short. For instance, such a minimalist approach would mask the just mentioned problem of "working poverty" that is of increasing importance for Germany. The phenomenon is caused by an increase in labour market flexibility through short-time work ("Kurzarbeit") and temporary work ("Leiharbeit" or "Zeitarbeit"). From 2008 to 2009, in the midst of the economic crisis, the number of short-term workers increased from about 100,000 to more than 1.1 million (Faik, 2012, p. 6). In addition, according to the Federal Employment Agency, more than 870,000 people were employed by one of almost 18,000 recruitment service companies by the end of 2011. This is a precarious situation since, again according to the Federal Employment Agency, temporary workers earn considerably less for the same type of work than those normally employed, leaving many dependent on additional social security 
benefits. Thus, the state in fact subsidises low wages and contributes to the problem of poverty in old age (Burmeister, 2012, p. 4).

In order to account for the working poor, a minimum wage comes in handy as a possible indicator. As Germany does not have an official minimum wage, I draw on a suggestion of the Hans Böckler Stiftung to utilise the limit of exemption from execution in order to derive an appropriate minimum wage (Böckler Impuls, 2006, p. 1). Until 1 July 2011, the limit of exemption from execution was 989.99 Euro, an amount that can be easily translated into a minimum wage of 8.29 Euro per hour ${ }^{32}$. In order to ensure the comparability of this amount over time, the value is indexed by the CPI (base year 2010).

Whereas the income aspect of employment has traditionally received a lot of attention, there exists another aspect that suffered chronic neglect: "time poverty". This is the third component of the deprivation indicator. Especially from a capability perspective, the ability to have a sufficient amount of leisure time at command is crucial as it is an elementary precondition for the ability to participate in social life. The aforementioned survey of families in Germany (Monitor Familienleben, 2012) highlights the importance of the topic in the German context. The majority of parents with children under the age of sixteen expressed their desire to be able to spend more $(45 \%)$ or even much more $(28 \%)$ time with their families. Only $23 \%$ of the respondents declared to have sufficient time for their families. I account for this fact in the following way.

The so called 'working hour tension' captures the disparity between actual and preferred working hours, whereby the related income changes induced by changes in working hours are explicitly taken into account. This indicator thus accounts for the fact that people might be over- or underemployed (Merz, 2002). The indicator is based on two questions in the GSOEP that question respondents about i) their actual weekly working hours, and ii) the weekly hours they would prefer to work if explicitly accounting for the fact that income changes with working hours. Working hour tension is then calculated as the difference between actual and preferred working hours. In the following, I will consider an individual as time poor if his or her working hour tension is ten hours or more.

The fourth dimension is housing, directly capturing capabilities number two, 'bodily health' and seven, 'affiliation'. Of course the requirement to have adequate shelter is especially important from the perspective of the 'affiliation' capability. In fact, it is one of the

\footnotetext{
${ }^{32}$ This is a monthly wage of 1,370 Euro (based on a 38-hour work-week) which is higher than the official minimum wage of the United Kingdom (1,202 Euro), but considerably lower than the official minimum wages of France (1,398 Euro), Belgium (1,444 Euro), Netherlands (1,447 Euro), Ireland (1,462 Euro), and Luxembourg (1,802 Euro).
} 
perspectives in which the capability approach is most convincing. In order to be able to participate in the social life of the community, a certain minimum standard of living is absolutely necessary. Children who are ashamed of their living conditions will deliberately prevent any relationship from becoming more than superficial as they are afraid that the relationship might reach a point where they would be forced to invite someone home. These children grow up isolated and deprived of social contacts that would be crucial for their development. In order to capture this aspect, I follow the suggestions of Arndt and Volkert (2007) and use the following three indicators: "unacceptable housing", "lack of socially necessary amenities", and "overcrowding".

I again follow Arndt and Volkert (2007) by characterising anyone as deprived according to "unacceptable housing" whose housing is characterised as either "in urgent need of complete renovation' or 'in danger of breaking down' (Arndt and Volkert, 2007, p. 28). Persons are identified as deprived according to a "lack of socially necessary amenities", if they lack either of the following "in-house bath/shower", "in-house toilet", "warm water", "central heating" (Arndt and Volkert, 2007, p. 28). However different from Arndt and Volkert (2007) out of aforementioned reasons, I refrain to utilise the subjective notion of overcrowding as the threshold level. Instead, persons are considered to suffer from "overcrowding" if their living space is below which was, at least until 2010, the appropriate living space for those receiving welfare payments under the so-called Hartz-IV-scheme: $45 \mathrm{sqm}$ for the first and $15 \mathrm{sqm}$ for every additional person (infants below two years of age excluded).

The fifth dimension is mobility, capturing basically capabilities three, 'bodily integrity', and seven, 'affiliation'. Mobility is increasingly a prerequisite for pursuing a profession. Especially in more rural areas many people have to commute out to find work, in addition many couples have not been able to find work in the same city, requiring them to commute. Mobility is also a precondition for the ability to participate in social life, all the more in case children are concerned who are especially vulnerable. Mobility can be restricted due to i) limited access to transportation and/ or ii) an insecure environment. ${ }^{33}$

The first aspect is captured by the "inability to afford a much-needed car". In the cities, mobility is usually ensured by public transportation systems; however, this system is often not very well developed outside of towns. Thus, a person is considered deprived in this indicator

\footnotetext{
${ }^{33}$ Please note that a bad health condition could also limit a person's mobility and could therefore be included as a third indicator. I nevertheless refrain to do so in order to prevent double-counting as a bad health condition is already included in the health dimension.
} 
if he or she lives in a household without a car and public transport is more than 20 minutes away.

The second aspect is captured by "insecure environment". A person is considered to be deprived according to this indicator, if he or she lives in an environment that is classified as either "insecure" or "dangerous". A disadvantage of this indicator is that the GSOEP retrieves this information only every five years, thus the inclusion of this indicator creates missing values whenever there is no information about a person's whereabouts in the years for which the respective question was not included in the survey.

The sixth dimension is environment, capturing parts of capability eight, 'other species'. This dimension is again captured by two indicators, "noise" and "pollution". A person is considered to be deprived with regard to "noise" if he or she suffers strong or very strong impairments through a very noisy neighbourhood. Similarly, a person is considered to be deprived with regard to "pollution" if he or she suffers strong or very strong impairments through a heavily polluted neighbourhood. As in the case of "insecure environment" these two indicators are retrieved only every five years, thus their inclusion again creates missing values whenever there is no information about a person's whereabouts in the years for which the respective question was not included in the survey.

Finally, the seventh dimension is income, capturing directly capability number ten, 'control over one's environment'. Though income is definitely not the only indicator for poverty measurement, it is obviously an important one. I will consider a person as income deprived if his or her disposable (i.e. after debt service) household income is below the official German breadline as defined in the seventh Existenzminimumbericht (breadline report) for 2010, i.e. below 638 EUR for single persons; 1,083 for couples; and 322 EUR for each child. As the issue of additional persons in the household is not captured by the report, I utilise the difference in the amount allowed to single persons and to couples, i.e. 356 EUR, for each additional adult in the household. In order to ensure the comparability of the breadline over time, the value is indexed by the CPI (base year 2010). Please note that these figures are indeed breadline figures that happen to be considerably below those of the AROPR.

Figure 3.01 provides an overview of the different dimensions, indicators and thresholds used for the calculation of the GCSPI.

Figure 3.01 The Structure of the GCSPI

\begin{tabular}{|c|c|c|c|}
\hline Dimension & Main Capability & Indicator & Threshold \\
\hline \multirow{2}{*}{ Health } & \multirow{2}{*}{ Bodily Health } & $\begin{array}{l}\text { Health } \\
\text { Condition }\end{array}$ & Subjective health condition either poor or bad \\
\hline & & $\begin{array}{l}\text { Health } \\
\text { Impairments }\end{array}$ & $\begin{array}{l}\text { At least four of the following: } \\
\text { 1. Have trouble climbing stairs }\end{array}$ \\
\hline
\end{tabular}




\begin{tabular}{|c|c|c|c|}
\hline & & & $\begin{array}{l}\text { 2. Health limits vigorous activities } \\
\text { 3. Achieved less due to physical health condition } \\
\text { 4. Achieved less due to mental health condition } \\
\text { 5. Reduced social contacts due to health problems }\end{array}$ \\
\hline \multirow{2}{*}{ Education } & \multirow{2}{*}{$\begin{array}{l}\text { Senses, } \\
\text { Imagination and } \\
\text { Thought }\end{array}$} & Schooling & Less than nine years of schooling \\
\hline & & Graduation & Neither graduation nor training qualification \\
\hline Employment & $\begin{array}{l}\text { Affiliation } \\
\text { Control over } \\
\text { Environment } \\
\text { Play }\end{array}$ & Employment & $\begin{array}{l}\text { At least one of the following: } \\
\text { 1. Activity status 'unemployed' } \\
\text { 2. Working poor (wage below minimum) } \\
\text { 3. Time poor (working hour tension at least } 10 \text { hours) }\end{array}$ \\
\hline \multirow{3}{*}{ Housing } & \multirow{3}{*}{$\begin{array}{l}\text { Bodily Health } \\
\text { Affiliation }\end{array}$} & $\begin{array}{l}\text { Housing } \\
\text { Condition }\end{array}$ & $\begin{array}{l}\text { Condition of housing either: } \\
\text { 1. In urgent need of complete renovation } \\
\text { 2. In danger of breaking down }\end{array}$ \\
\hline & & $\begin{array}{l}\text { Socially } \\
\text { Necessary } \\
\text { Amenities }\end{array}$ & $\begin{array}{l}\text { Lack of either of the following: } \\
\text { 1. In-house bath / shower } \\
\text { 2. Warm water } \\
\text { 3. In-house toilet } \\
\text { 4. Central heating }\end{array}$ \\
\hline & & Living Space & $\begin{array}{l}\text { Living space below minimum ( } 45 \mathrm{sqm} \text { for first, } 15 \mathrm{sqm} \\
\text { for every additional household member (infants } \\
\text { excluded)) }\end{array}$ \\
\hline \multirow{2}{*}{ Mobility } & \multirow{2}{*}{ Bodily Integrity } & Transport & $\begin{array}{l}\text { Public transport more than } 20 \text { minutes away and no car } \\
\text { available }\end{array}$ \\
\hline & & Crime & Neighbourhood either insecure or dangerous \\
\hline \multirow{2}{*}{ Environment } & & Pollution & Strong or very strong impairments through pollution \\
\hline & & Noise & Strong or very strong impairments through noise \\
\hline Income & $\begin{array}{l}\text { Control over } \\
\text { Environment }\end{array}$ & $\begin{array}{l}\text { Disposable } \\
\text { Income }\end{array}$ & $\begin{array}{l}\text { Disposable income below breadline ( } 638 \text { EUR for first, } \\
356 \text { EUR for every additional adult, } 322 \text { EUR for } \\
\text { every additional child per household) }\end{array}$ \\
\hline
\end{tabular}

Once dimensions, indicators and threshold levels have been chosen, the next exercise concerns the choice of weights for dimensions and indicators. Several options can be applied in order to choose the weights. As far as the dimensions are concerned, I utilise a rather conservative approach by applying equal weights, i.e. each dimension contributes to overall deprivation in the same way. The considerations leading up to that decision are primarily motivated by the fact that the dimensions are directly derived from Martha Nussbaum's list of central human capabilities, thus it seems somewhat inappropriate to utilise different weights for them - at least as long as no participatory approach is available that would provide a convincing basis for a deviation.

Things are different for the choice of indicators. In that case, I apply two different approaches. The first one is again the equal weighting approach, i.e. each indicator contributes to the respective poverty dimension in the same way. The second approach is called prevalence or frequency-based weighting. With this approach, each indicator is weighted in dependence of the proportion of the individuals in the population who are not deprived in that indicator at each point in time. The higher the proportion of those who are not deprived in a given indicator, the higher is the weight assigned to it. The reasoning behind this approach is 
that the lower the likelihood that a person is deprived in an indicator, the more he or she has reason to feel deprived. Thus, the higher weight acknowledges the stronger indicative nature of this specific indicator with regard to deprivation. Moreover, as prevalence weights are calculated for each point in time, this weighting approach is able to account for a situation in which the condition of a person does not change although the overall situation in the society improves: as the proportion of those not deprived in a given indicator increases, the weight of that indicator increases as well, implying a ceteris paribus increase in the deprivation score of such person (e.g. Figari, 2012).

Due to its appealing nature, I will base the following analysis on the prevalence weighted GCSPI unless stated otherwise. However, in order to test the robustness of the results, all results were calculated for the equal weighting approach as well. The results for both equal as well as prevalence weighted GCSPI for the years 2002-2010 can be found in tables 3.05 to 3.14 in the appendix. They are highly correlated, as the following table demonstrates.

Table 3.01 Spearman Rank Correlation Equal and Prevalence Weights

\begin{tabular}{|c|c|c|c|c|c|}
\hline & 2002 & 2004 & 2006 & 2008 & 2010 \\
\hline Spearman correlation & 0.9980 & 0.9982 & 0.9980 & 0.9980 & 0.9979 \\
\hline Number obs. & 15,564 & 18,341 & 14,211 & 15,326 & 14,672 \\
\hline $\mathrm{p}$ value & 0.0000 & 0.0000 & 0.0000 & 0.0000 & 0.0000 \\
\hline $\begin{array}{l}\text { Spearman correlation } \\
\text { (obs. different from zero) }\end{array}$ & 0.9934 & 0.9940 & 0.9933 & 0.9928 & 0.9922 \\
\hline Number obs. & 10,382 & 12,198 & 9,433 & 9,933 & 9,436 \\
\hline $\mathrm{p}$ value & 0.0000 & 0.0000 & 0.0000 & 0.0000 & 0.0000 \\
\hline
\end{tabular}

Spearman rank correlation coefficients range from 0.9979 (2010) to 0.9982 (2004) considering all observations, and from 0.9922 (2010) to 0.9940 (2004) considering only those observations for which the index is larger than zero. Also, the respective ranking of the Bundeslaender is the same for both weighting methods with only very few minor exceptions $^{34}$.

Figure 3.02 Sum of Missing Values 2002-2010

\begin{tabular}{llrrrrr}
\hline Dimension & Indicator & 2002 & 2004 & 2006 & 2008 & 2010 \\
\hline \multirow{2}{*}{ Health } & Health Condition & 4,257 & 3,506 & 4,103 & 4,013 & 4,801 \\
\cline { 2 - 7 } & Health Impairments & 419 & 357 & 568 & 485 & 442 \\
\hline \multirow{2}{*}{ Education } & Schooling & 1,201 & 1,238 & 1,197 & 1,061 & 953 \\
\cline { 2 - 7 } & Graduation & 0 & 0 & 0 & 0 & 0 \\
\hline \multirow{2}{*}{ Employment } & Employment & 254 & 251 & 285 & 247 & 266 \\
\cline { 2 - 7 } Housing & Housing Condition & 59 & 78 & 55 & 40 & 66 \\
\cline { 2 - 8 } & Socially Necessary Amenities & 211 & 230 & 0 & 1 & 0 \\
\hline
\end{tabular}

\footnotetext{
${ }^{34}$ When changing from the equal weighting approach to prevalence weighting, there are six minor rank changes, one in 2002 (Hesse, initially rank 14, switches places with Saxony, initially rank 15); two in 2004 (Bavaria, initially rank 5, switches places with North Rhine-Westphalia, initially rank 6; Hesse, initially rank 11, switches places with Saxony, initially rank 10), one in 2006 (Bavaria, initially rank 7, switches places with Hesse, initially rank 8) and two in 2008 (Bremen, initially rank 3, switches places with Lower Saxony, initially rank 2; Saarland, initially rank 14, switches places with Saxony-Anhalt, initially rank 15).
} 


\begin{tabular}{llrrrrr}
\hline & Living Space & 0 & 0 & 0 & 0 & 0 \\
\hline \multirow{2}{*}{ Mobility } & Transport & 826 & 14 & 858 & 268 & 293 \\
\cline { 2 - 7 } & Crime & 4,248 & 81 & 4,470 & 1,261 & 1,032 \\
\hline \multirow{2}{*}{ Environment } & Pollution & 44 & 47 & 34 & 50 & 40 \\
\cline { 2 - 7 } & Noise & 10 & 6 & 8 & 10 & 9 \\
\hline \multirow{2}{*}{ Income } & Disposable Income & 1,014 & 1,334 & 943 & 1,166 & 1,335 \\
\hline Sum & & 12,543 & 7,142 & 12,521 & 8,602 & 9,237 \\
\hline Original Adult Sample Size & 28,107 & 25,438 & 26,732 & 23,928 & 23,909 \\
\hline
\end{tabular}

In order to get a first impression how the GCSPI works in practice, i.e. when applied to real data, it is crucial to compare the results with those of other poverty indices. This chapter will utilise two other poverty indices besides the GCSPI that are based on the two main theories of welfare economics, i.e. the income approach and traditional welfarism that defines well-being in terms of happiness. Thus, the following section will start with a brief introduction of these two indices.

\subsection{Empirical Application}

The first index is the AROPR that is based on the theoretical approach to measure poverty as a lack of income and is the only official index used to evaluate poverty in Germany. The results for the AROPR for the years 2002-2010 can be found in table 3.04 in the appendix.

The second index belongs to the group of happiness indices that recently received a lot of attention. This development is rather astonishing considering the fact that the corresponding theory, i.e. traditional welfarism, had been abandoned just because of the impossibility of inter-personal happiness comparisons (Robbins, 1938, p. 640; Sen, 2009, p.277-278). The recent interest in happiness indices appears like a late resurrection of the traditional approach.

Aristotle's concept of happiness differentiates between instant feelings on one hand and long-term happiness one the other, claiming that only the latter, the so called 'Eudaimonia' (Bartlett and Collins, 2012, p. x) is adequate for evaluation. Thus, the hereafter introduced Subjective Correlation Sensitive Poverty Index (SCSPI) is based on questions related to individual satisfaction, reflecting the conviction that the concept of satisfaction comes very close to the concept of 'Eudaimonia' that Aristotle suggested for evaluation. Methodologically, the SCSPI is based on the same mathematical formula (1) as the GCSPI, thus satisfies the same properties.

Different from the GCSPI, the SCSPI is based on only four dimensions: 1. health, 2. employment, 3. housing, and 4. income. The other three dimensions of the GCSPI, i.e. education, mobility and environment, could not be included due to a lack of data. The SCSPI is based on a self-evaluation on a scale ranging from zero (completely dissatisfied) to ten 
(completely satisfied). The chosen threshold level for all the dimensions is three, i.e. considerably dissatisfied. Figures 3.03 to 3.06 in the appendix illustrate the distribution of responses across the German population for the year 2010.

Please note that the same classification of poverty degrees is used in the case of the SCSPI that has been introduced for the GCSPI. Again, category one describes those who are deprivation affected, i.e. those whose weighted sum of deprivations is one third or less. As in the case of the GCSPI, the responding headcount will not be considered in the following analysis. Category two defines those who are poor, i.e. those whose weighted sum of deprivations is higher than one third but not higher than two thirds. Finally, category three describes the severely poor, i.e. those whose weighted sum of deprivations is higher than two third. The results for the SCSPI for the years 2002-2010 can be found in tables 3.15 to 3.19 in the appendix.

As described in the introduction, both theoretical approaches to poverty measurement, traditional welfarism as well as the income approach, received a lot of criticism over the years. They left a theoretical gap to be filled, a gap that motivated Amartya Sen to introduce a whole new theory of welfare economics, i.e. the capability approach on which the GCSPI is based. The following analysis addresses the question whether and if yes in how far the differences in the theoretical approaches do indeed make a difference "on the ground". In other words, when applied to real data, do the evaluations of poverty and poverty trends really differ? Is there indeed a need for the GCSPI?

For a start, the following table provides the results of the statistical correlations between the poverty dimensions of GCSPI, SCSPI, and AROPR based on the respective Kendall tau $b$ correlations for 2010 (Number of observations: 8,534).

Table 3.02 Kendall Tau b Correlation all Dimensions

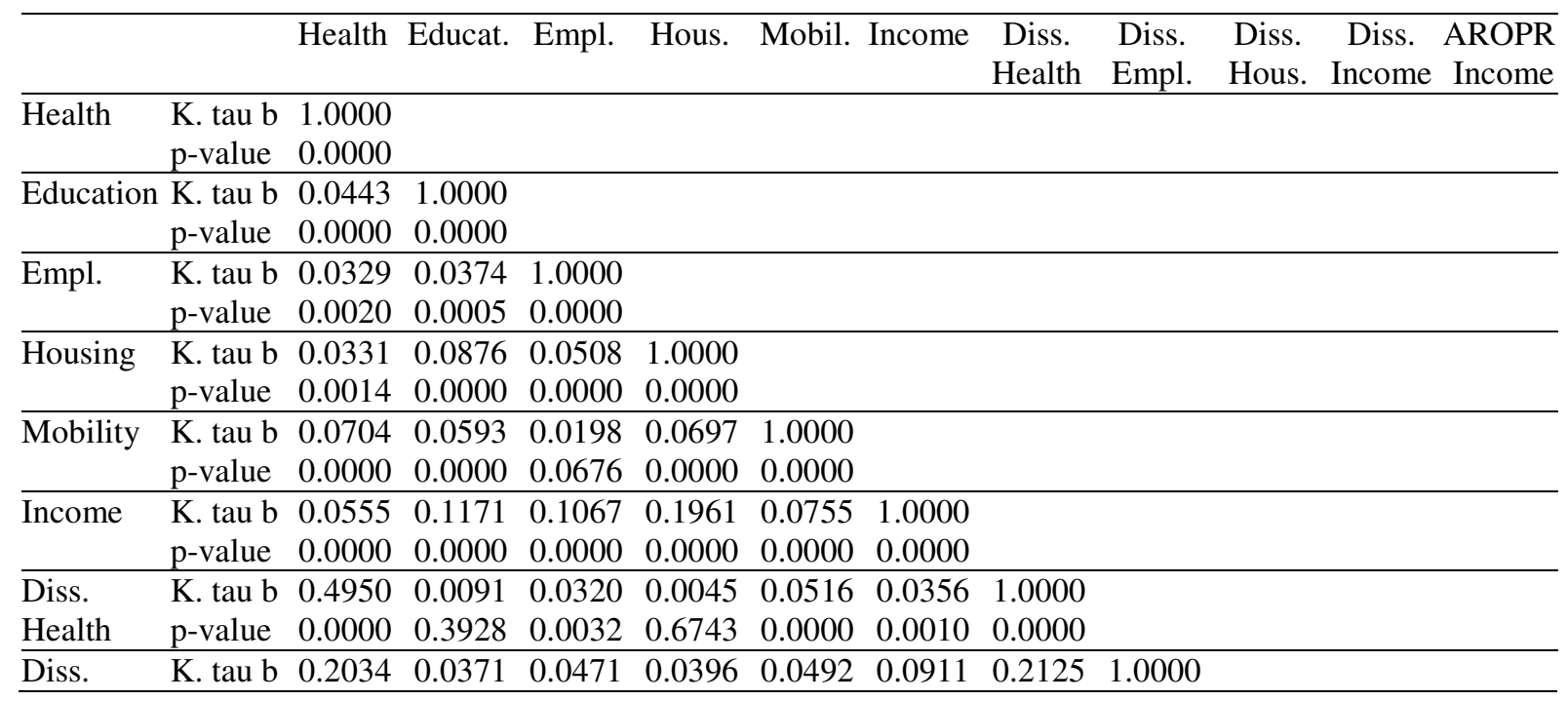




\begin{tabular}{|c|c|c|c|c|c|c|c|c|c|c|c|c|}
\hline Empl. & $\mathrm{p}$-value & 0.0000 & 0.0005 & 0.0000 & 0.0002 & 0.0000 & 0.0000 & 0.0000 & 0.0000 & & & \\
\hline Diss. & K. tau b & 0.1055 & 0.0463 & 0.0462 & 0.1592 & 0.0936 & 0.0827 & 0.1253 & 0.1202 & & & \\
\hline Housing & $\mathrm{p}-\mathrm{v}$ & 0.0000 & 0.0000 & 0.0000 & 0.0 & 0.0000 & 0.0000 & 0.0000 & 0.0000 & & & \\
\hline Diss. & K. t & 0.1924 & 0.0624 & 0.0957 & 922 & 764 & 009 & 821 & 0.2694 & 812 & & \\
\hline Income & p-value & 0.0000 & 0.0000 & 0.0000 & 0.0000 & 0.0000 & 0.0000 & 0.0000 & 0.0000 & 0.0000 & 0.0000 & \\
\hline$\overline{\mathrm{AROPR}}$ & K. tau b & 0.0650 & 0.1031 & & & & & & 0.1008 & 0.0807 & 85 & \\
\hline Income & p-value & 0.0000 & 0.0000 & 0.0000 & 0.0000 & 0.0000 & 0.0000 & 0.0001 & 0.0000 & 0.0000 & 0.0000 & 0.0000 \\
\hline
\end{tabular}

p-values correspond to the null hypothesis that the two indicators are independent.

All poverty dimensions demonstrate a positive correlation - though not always statistically significant - that indicates that they all indeed measure the same thing, i.e. poverty. At the same time, Kendall tau $\mathrm{b}$ is considerably lower than 0.80 in all cases except for the correlation between the breadline and AROPR, demonstrating that each dimension measures a distinctively different aspect of poverty.

It is interesting to note that the strongest correlation exists between the different dimensions of dissatisfaction. It might point to a problem with subjective evaluation: a person that is dissatisfied in one dimension is rather likely to transfer this dissatisfaction to other dimensions as well. Imagine, for instance, a person suffering from a bad health condition that might be as severe as to even limit his or her ability to move freely. Or imagine a person suffering from a bad mental condition. It seems rather likely to suggest that the overall bad feeling of the person is not only reflected in questions directly related to health but as well with regard to any other dimension. This would explain why the correlation between the two dimensions health and housing is rather low, as would be expected, whereas the correlation between dissatisfaction with health and dissatisfaction with housing is non-negligible.

What is also rather interesting is the fact that, while the correlation between income and all other poverty dimensions is always highly significant, it is at the same time in many cases astonishingly low. Especially interesting is the fact that the correlation between income and dissatisfaction with income is highly significant but much weaker than one would expect at first sight. This supports the fact that though income is of course correlated with other poverty dimensions, it is not an equally good proxy for all of them.

These findings are also supported by the following figure that compares the number of persons who are deprived according to the AROPR with those who are deprived according to the six non-income dimensions of the GCSPI (number of observations: 14,655). 
Figure 3.07 Headcounts AROPR and GCSPI

$\begin{array}{ll}\text { Overlap of AROPR and: } & \\ \text { - Environment: } & 117(9 \%) \\ \text { - Mobility: } & 192(14 \%) \\ \text { - Education: } & 420(31 \%) \\ \text { - Health: } & 533(39 \%) \\ \text { - Employment: } & 577(42 \%) \\ \text { - Living Conditions: } & 595(44 \%)\end{array}$

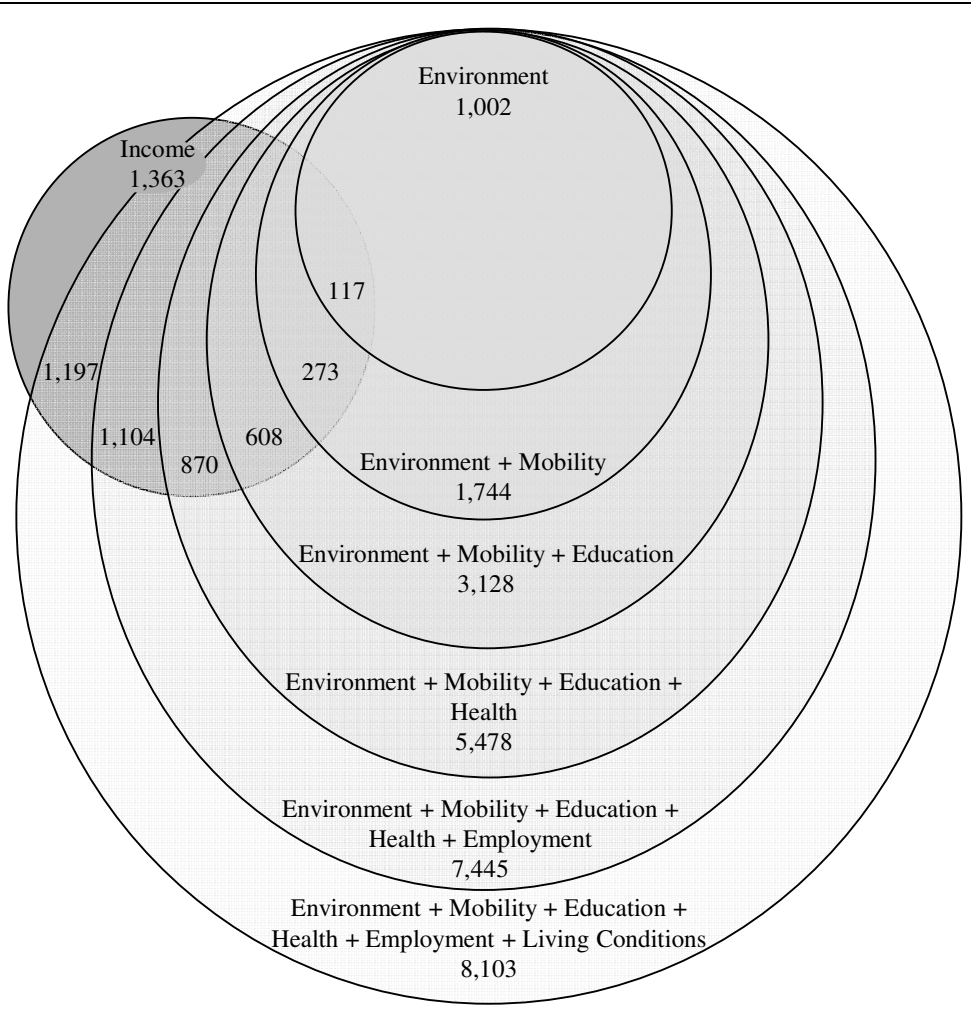

As one can easily see, the overlap - or the match - between the population parts that are at-the-risk-of-poverty and those that are deprived in the different non-income dimensions of the GCSPI is not at all distinct. In fact, the figures reveal a considerable failure of the income approach to account for other poverty dimensions apart from income. As the low Kendall tau b correlation coefficients already indicate, the congruence between those who are deprived in income and those who are deprived in the non-income GCSPI dimensions ranges between merely $9 \%$ (environment) and 44\% (living conditions).

What the figure also captures is the fact that even after accounting for all six non-income GCSPI dimensions, there are still individuals left who are not deprived in any of these dimensions but are still at-the-risk-of-poverty. As has already been mentioned, the AROPR is considerably higher than the official breadline. Thus, I additionally verify whether there are also individuals whose net equivalence income is below the official breadline but who are nevertheless not deprived in any of the six non-income GCSPI dimensions. This is indeed the case: overall, $6.8 \%$ of the respondents have a net equivalence income below the official breadline. Of those, $12.4 \%$ are not captured by any of the six non-income GCSPI dimensions. This observation provides additional support for the decision to include income as a poverty dimension in the GCSPI - apart from the theoretical argument that it is the main indicator able to capture capability number ten. 
The question that inevitably comes to mind when considering the weak correlation between the dimensions of the three poverty indices is how this affects poverty measurement. The following figure provides the deprivation headcounts (categories two and three) for GCSPI, SCSPI and AROPR for the German Bundeslaender. Please note that in this specific case all indices refer to the same persons. All observations have been dropped that did not provide enough information to calculate all three indices.

Figure 3.08 Headcounts GCSPI, SCSPI and AROPR Germany 2010

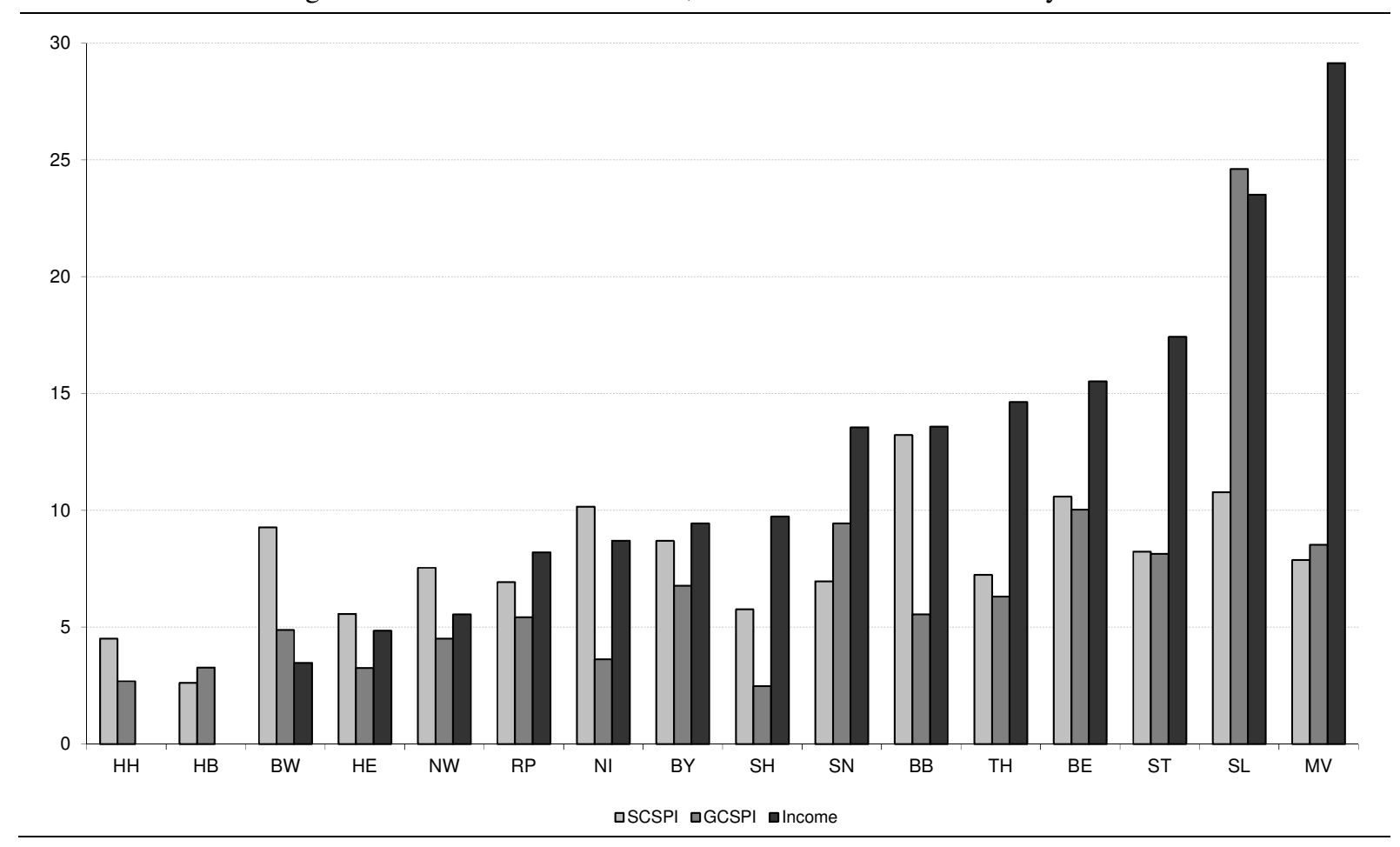

The first thing that immediately strikes the eye is the considerable discrepancy between old and new Bundeslaender, i.e. West and East Germany. All three indices yield comparably higher values for the Eastern Bundeslaender but their relevance for the different parts of Germany is rather different. AROPR plays a much stronger role in the Eastern Bundeslaender whereas the SCSPI plays a much stronger role in the Western Bundeslaender, especially in those that are more populous and urbanised. In fact, the figure almost acts like a mirror reflecting these two different trends.

In order to get a more dynamic impression of how the different approaches to measurement may differ, the following figure compares them over time and within the context of the development of the most important figures of the German economy. Please note that in this figure GCSPI and SCSPI are illustrated, not just their headcounts. 
Figure 3.09 Development of Economic Figures Germany 2002-2010

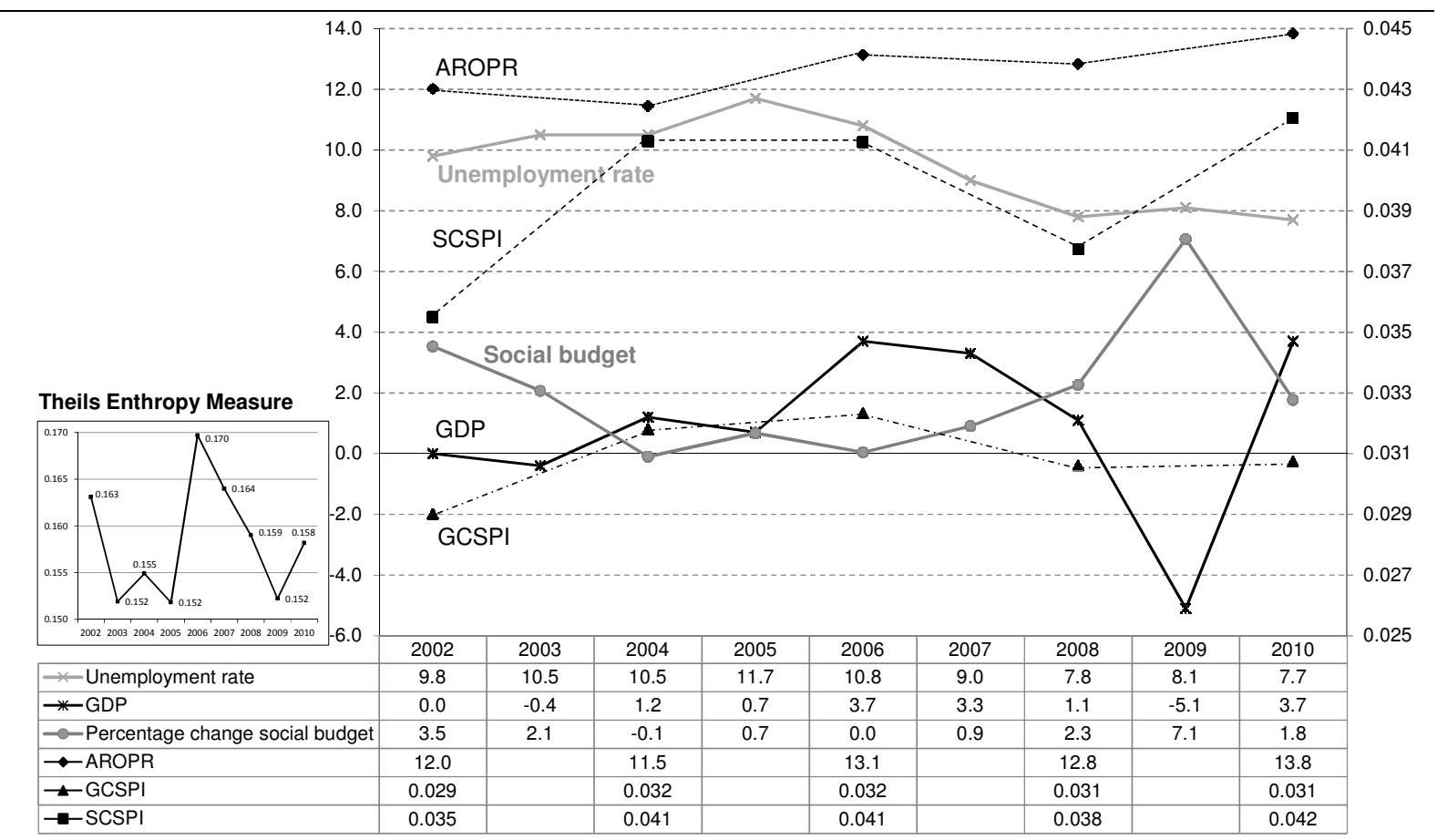

The first thing to mention is the obvious volatility of the SCSPI over time, just as one would expect to be the case for subjective indices. It seems that the SCSPI is so busy in reflecting short-term changes that it is actually unable to provide an overall trend. The other two indices, the AROPR and the GCSPI, are able to provide such an overall trend. However, the trends they indicate diverge during two very interesting time periods, i.e. between 2002 and 2004 and between 2008 and 2010. The reason why these two time periods are especially interesting is that they capture both economic crises that Germany faced during the considered time period, i.e. one in 2003 and one in 2009.

As Faik (2012, p. 8) points out, both crises are insofar comparable as income inequality decreased during the crisis, followed by an increase in the following year when the economy recovered. It is a typical outcome of a social welfare state whose social security system cushions the effect of the economic crises for the poorest parts of the population whereas the wealthiest parts typically experience its full force. In order to get an impression of the development of income inequality over time, I included a picture of the development of Theil's enthropy measure over time. The measure belongs to the same class of GE inequality measures that is utilised in the GCSPI, though in this case for $\theta=1^{35}$ :

\footnotetext{
${ }^{35}$ Any other value of $\theta$ would already imply an evaluation of inequality - like in the formula the GCSPI uses which belongs to the same class of GE inequality measures but for $\theta \neq 0,1$. The purpose here, however, is a description, not an evaluation, of the development of income inequality in Germany over time.
} 


$$
T=\frac{1}{n} \sum_{i=1}^{n}\left[\frac{y_{i}}{\mu} \cdot \log \left(\frac{y_{i}}{\mu}\right)\right]
$$

with $y_{i}$ representing the equivalent income for person $i$ and $\mu$ representing the arithmetic mean of equivalent incomes. Data are taken from Faik (2012, p. 9).

Despite the same trends in income inequality during the two time period 2002-2004 and 2008-2010, there are two important differences. During the first recession in 2003, the percentage change of the social budget was reduced (from 3.5\% in 2002 to $2.1 \%$ in 2003) whereas it was significantly increased during the second recession in 2009 (from $2.3 \%$ in 2008 to $7.1 \%$ in 2009). ${ }^{36}$ Also, the unemployment rate happened to be higher after the first crisis, increasing from $9.8 \%$ in 2002 to $10.5 \%$ in 2004, whereas it was slightly lower after the second crisis, decreasing from $7.8 \%$ in 2008 to $7.7 \%$ in 2010 . Whatever induced these differences, they provide a rather unique opportunity to compare the way of functioning of the AROPR and the GCSPI.

During the first economics crisis (2002-2004), rising unemployment and the decline in the percentage change in social benefits together with the implications of the crisis provide a more than convincing explanation for the noticeable increase in the GCSPI. The AROPR, however, fell slightly during the same time period. This can only be explained if the trend in inequality is taken into account: the loss of the wealthier parts of the society has been stronger than the loss of the poorer parts, causing a reduction in the income inequality of the society as a whole. This is reflected in a decrease of the AROPR. To put it more plainly, the AROPR decreases not because of an improvement in the living conditions of the poor but because the deterioration in the living conditions of the poor was weaker than the deterioration in the living conditions of the wealthy.

In the case of the second, much more severe, economic recession and completely different from the first, the AROPR increases, reflecting again the development of overall inequality in the society that is in 2010 almost as high as in 2008. Just as in the case of the recession of 2003, the GCSPI shows a different trend: the GCSPI remains stable, capturing the slight decreases in the unemployment rate as well as in the social benefit expenditures between 2008 and 2010.

Other interesting stories could be told from the figure, for instance with regard to the steep increase in income inequality from 2005 to 2006, the year in which a new set of rules

\footnotetext{
${ }^{36}$ The reduction of the economic recession of 2003 is unusual but might be due to the fact that there had been a rather strong increase in the previous year's percentage change as the social budget was raised from almost 662 billion in 2001 to more than 685 billion in 2002, maybe an election gift.
} 
for the long-term unemployed and social welfare assistance was introduced, the Hartz IV regulations, to only name one. To tell them all, however, would go beyond the scope of this chapter.

I will commence the analysis with an illustration of the usefulness of the regional and dimensional decomposability of the multidimensional indices GCSPI and SCSPI. It is already apparent from figure 3.09 that the changes in the GCSPI have been rather minor over time. With this observation in mind, the question inevitably arises as to how able the index is to reflect changes in the living conditions of the poor. Is the reason for the minor changes only the result of an unwanted inflexibility of the poverty measure? In order to be able to respond to that so important question, I take advantage of the subgroup decomposability of GCSPI and compare the development of poverty rates across the Bundeslaender. Some of the results are illustrated in the following figure.

Figure 3.10 Different Poverty Paths in Germany 2002-2010

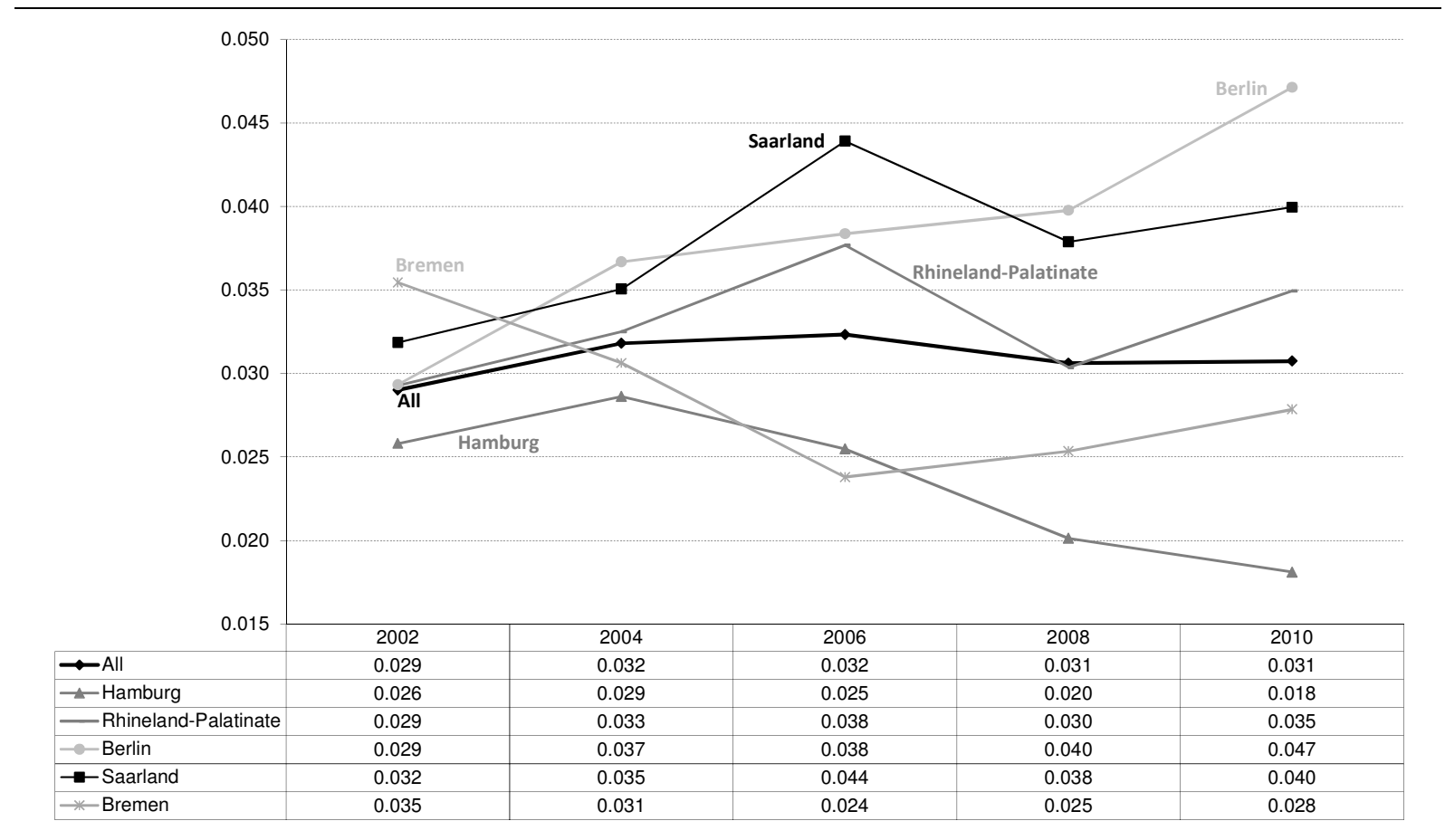

The figure provides evidence enough for the index's flexibility to react to changes in living conditions. The development of poverty has indeed been very diverse across the Bundeslaender, it highlights stories of success and failure that would be worth to be told, however go beyond the scope of this chapter. But one question that is related to these trends shall be studied in the following: in face of the obvious diversity of poverty trends are there also indications for an overall regional trend? Figure 3.08 already demonstrated that in 2010 
poverty levels in East Germany have been higher than in West Germany regardless of which poverty index was utilised. Are these differences stable or did they evolve over time?

Table 3.03 in the appendix compares the GCSPI as well as the respective decompositions according to poverty incidence, intensity and inequality across the Bundeslaender and over time. It seems indeed to indicate an overall regional trend. The maximum values for the overall index as well as all its three components increased over time and they originate in the vast majority of cases from Eastern Germany. The observation suggests that the picture drawn by figure 3.08 is not a coincidence but rather the result of a worrisome overall trend.

In order to shed further light on this first impression, the following figures provide poverty maps for Germany with regard to the AROPR, the GCSPI, and the SCSPI from 2002 to 2010 .

Figure 3.11 German Poverty Maps AROPR 2002-2010

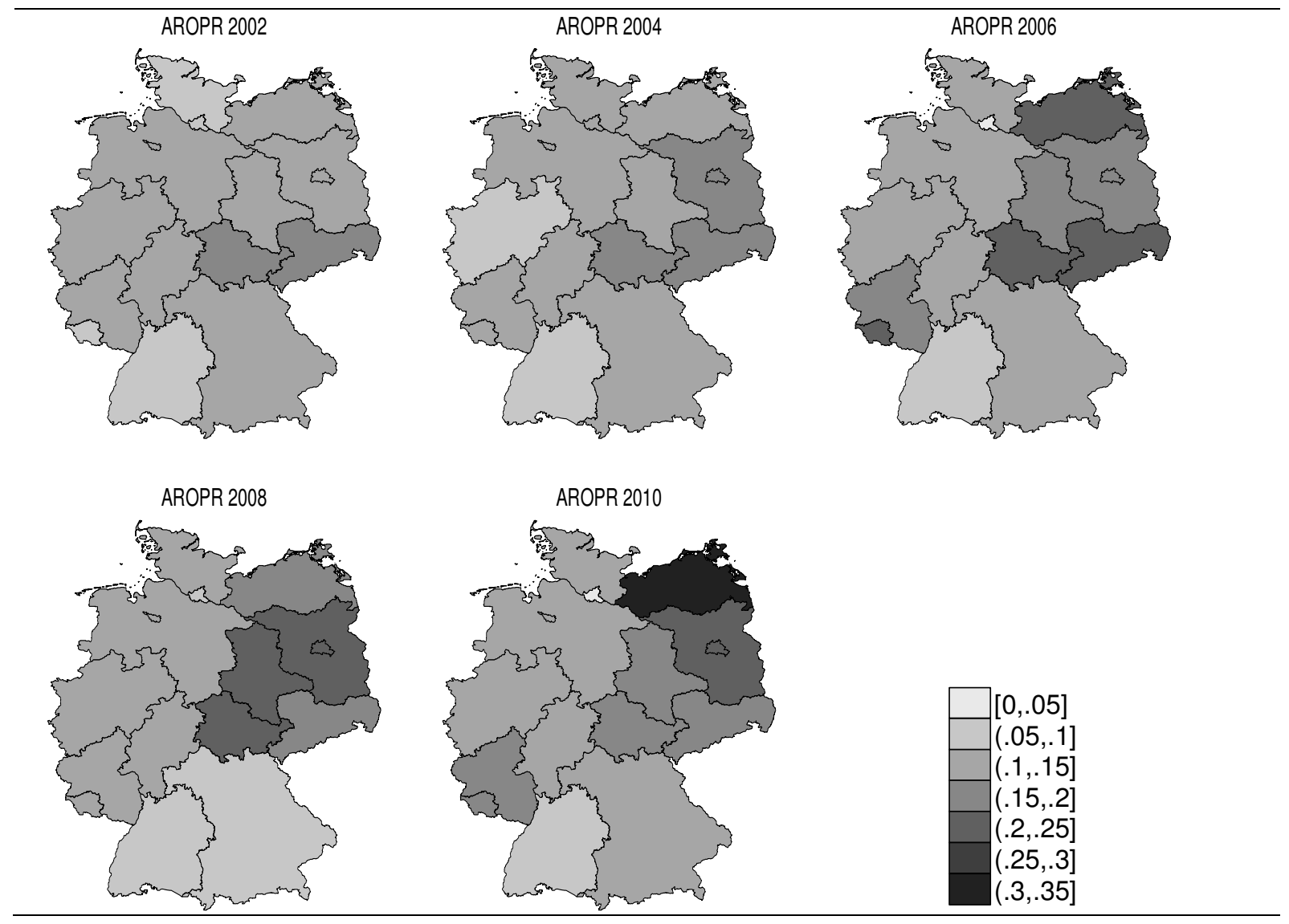


Figure 3.12 German Poverty Maps GCSPI 2002-2010
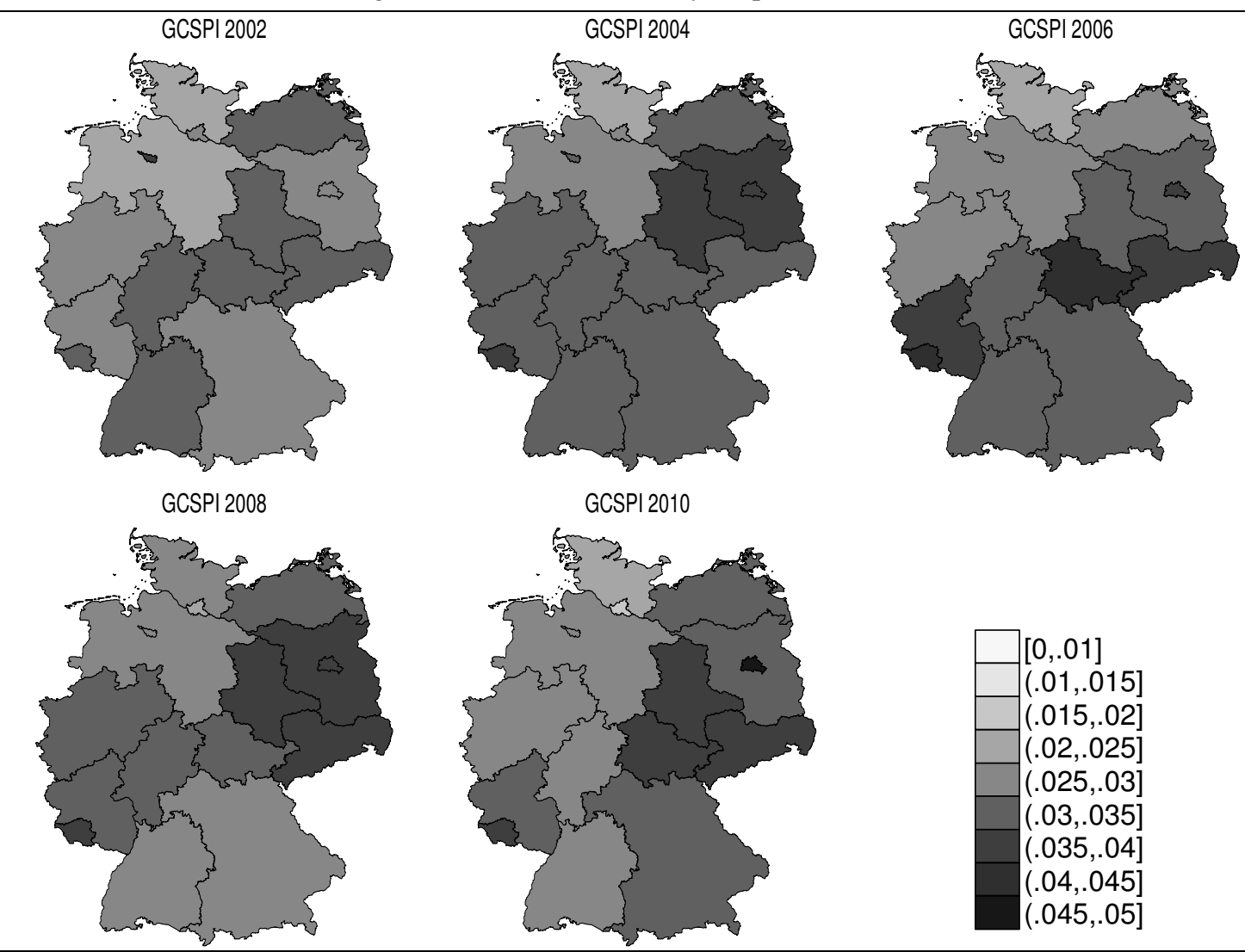

Figure 3.13 German Poverty Maps SCSPI 2002-2010

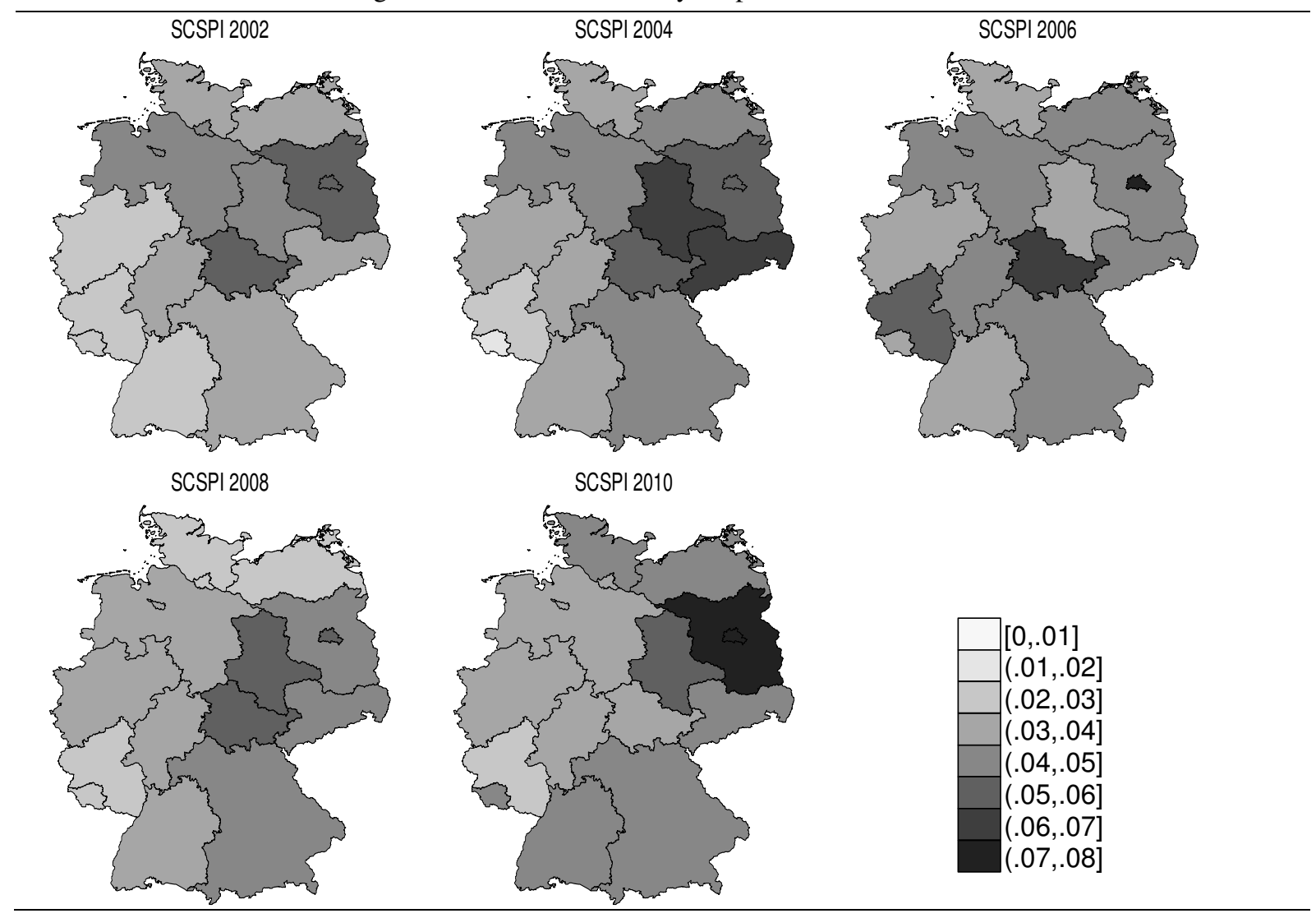


All poverty indices tell the same story: though there has only been a slight increase in overall poverty over time, a concentration process seems to have taken place that makes poverty more and more a problem of East Germany. However, there seems to be a small ray of hope: It seems as though in 2010 the worrisome trend weakened for the first time since 2002.

Considering the overall trend that the regional decomposition made visible, the first question that vies for attention is whether a similar overall trend can be detected with regard to dimensional decompositions. As has been pointed out before, the GCSPI as well as the SCSPI belong to the first class of additive poverty indices that satisfy the property of Factor Decomposability (FD). It is the fulfilment of that specific property that allows the decomposition of the indices according to poverty dimensions. Such decomposition is illustrated in the following figure for the GCSPI as well as the SCSPI across the Bundeslaender and over time.

Figure 3.14 Development of Dimensional Decompositions 2002-2010

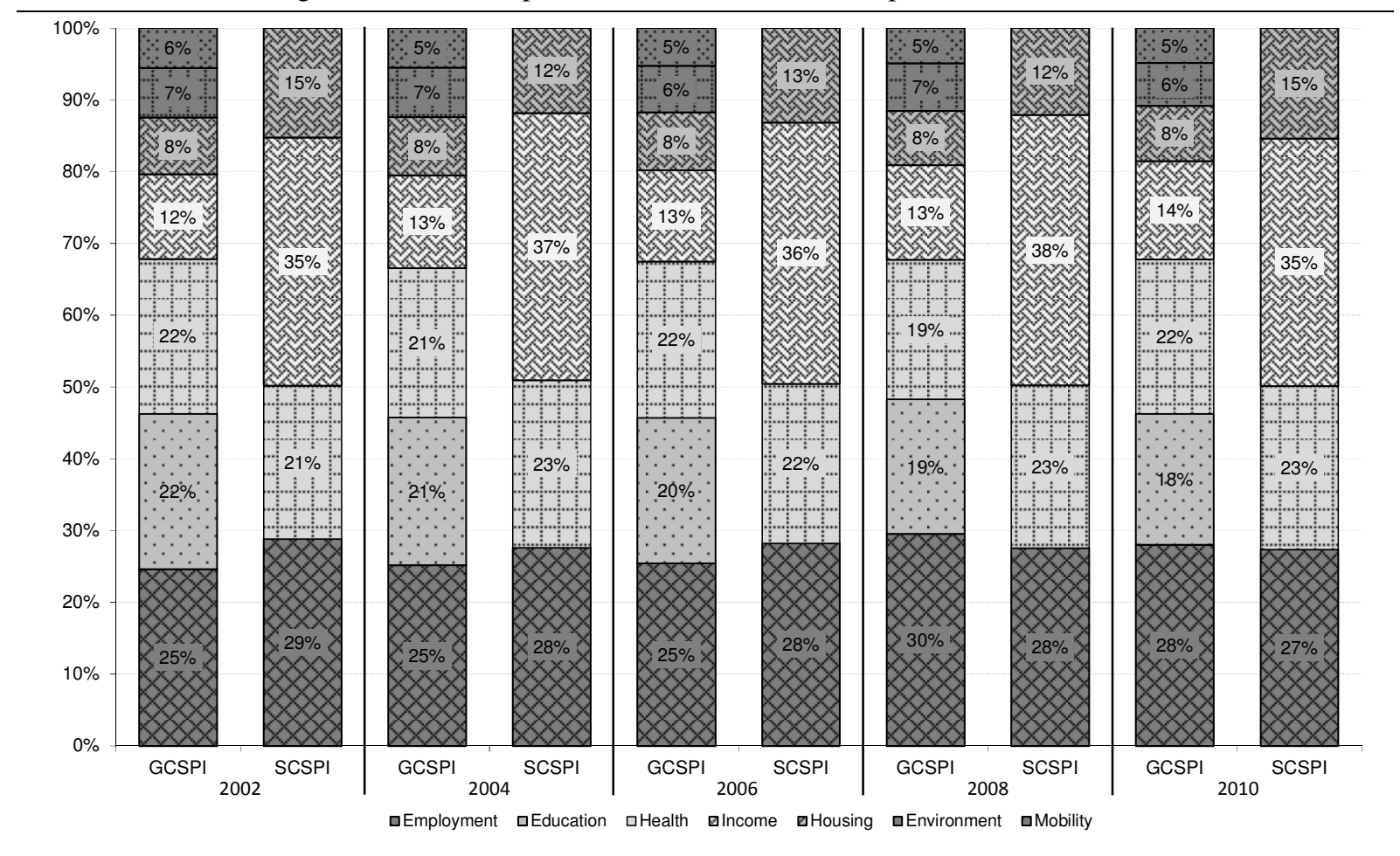

The high volatility of the SCSPI is also reflected in the dimensional decomposition. Clear trends in the contribution of the different dimensions are often not recognisable. One exception is the contribution of the dimension dissatisfaction with employment to overall poverty that shows a continuous decrease from $29 \%$ in 2002 to $27 \%$ in 2010 . The GCSPI, on the other hand, shows some clear trends. There exists a slight increase in the contribution of income, from $12 \%$ in 2002 to $14 \%$ in 2010, and a (stronger) decrease in the contribution of 
education, from $22 \%$ in 2002 to $18 \%$ in 2010. In addition, the contribution of employment shows a strong increase in the years 2008 and 2010, from 25\% in 2002 to $30 \%$ and 28\%, respectively. These trends have special significance if they are associated with the decomposition according to regions and dimensions which is illustrated by the following figure for 2010 .

Figure 3.15 Dimensional Decomposition of GCSPI 2010

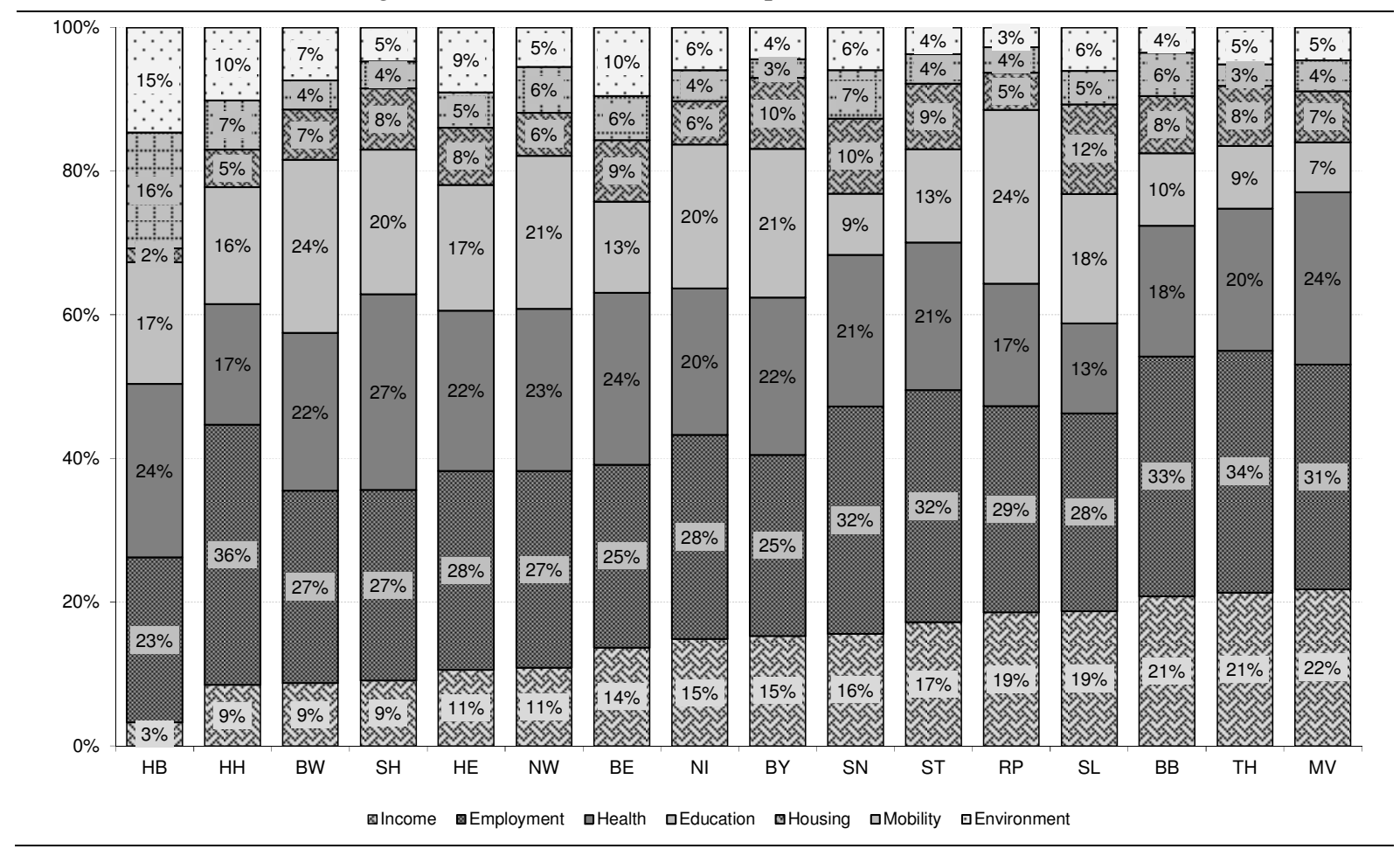

As expected, the dimension environment shows the strongest contribution in the urban areas, irrespective of whether they are located in the Eastern or Western parts of Germany (e.g. Berlin, Bremen, Hamburg). But the figure also indicates two regional peculiarities. It seems that in Eastern Germany income deprivation is a stronger contributor to overall deprivation compared to Western Germany, as already indicated by the stronger importance of the AROPR for this region (figure 3.08). In Western Germany, on the other hand, education seems to be the stronger contributor. This corresponds to the two different types of support programs of the European Social Funds in Germany. The Eastern Bundeslaender with the exception of Berlin - belong to the 'Convergence Regions', i.e. regions that are characterised by weak economic performance. The objective of the respective support programs is to speed up GDP growth in those regions so that they are able to catch up with the rest of Germany. The Western Bundeslaender belong - without exception - to the 'Competitiveness and Employment Regions'. The objective of the support programs in those 
regions is to promote life-long learning, training and to improve the reconciliation of work and family life.

If this regional peculiarity is associated with the trends in the dimensional decomposition it reveals two important sources for the pronounced drift apart in East and West German poverty levels as captured by the poverty maps: the declining significance of the contribution of educational deprivation, that has been the much stronger contributor to poverty in West Germany, combined with the increasing significance of the contribution of income deprivation that has been the much stronger contributor to poverty in East Germany.

By far not all stories have been told, the attempt would have gone well beyond the scope of this chapter. However, the stories that have been told all get to the same conclusion: the operationalisation of the capability approach by means of the GCSPI seems to be very worthwhile.

\subsection{Conclusions}

With the publication of the second German 'Poverty and Wealth' the German government adopted the decision to define poverty in Germany on the basis of the capability approach. However, so far the decision did not in any way impact on the way poverty is measured. Germany still utilises exclusively the at-risk-of-poverty rate (AROPR), defined as the population share with a net equivalence income below $60 \%$ of the median of the population, to assess poverty.

This chapter made a first suggestion to operationalise the capability approach by means of a multidimensional index, the German Correlation Sensitive Poverty Index (GCSPI). In addition, another subjective multidimensional poverty index is introduced, the Subjective German Correlation Sensitive Poverty Index (SGCSPI) that is based on the self-evaluation of satisfaction of the respondents - a procedure that became rather popular in recent years. The analysis revealed the high additional value that is generated in case the traditional incomebased AROPR is complemented by other, multidimensional poverty indices.

First, there is a strong discrepancy in the identification of the deprived according to the AROPR and the GCSPI. In fact, the analysis reveals a considerable failure of the income approach to account for other poverty dimensions apart from income: the congruence between those who are deprived in income and those who are deprived in the non-income GCSPI dimensions ranges between merely $9 \%$ and $44 \%$. In addition, even after accounting for all six non-income dimensions, there are still income-poor individuals left who are not deprived in 
any of these dimensions. And even in case the official breadline is used that is considerably lower than the AROPR there are individuals left whose net equivalence income is below the official breadline but who are nevertheless not deprived in any of the six non-income GCSPI dimensions: $12.4 \%$ of the $6.8 \%$ of the respondents with a net equivalence income below the official breadline are not captured by any of the six non-income GCSPI dimensions.

Second, there exists a considerably discrepancy in the regional composition of the three indices. The poverty rates provided by the AROPR and the SCSPI for the Western and the Eastern Bundeslaender seem to be an almost perfect reflection of each other. With only few exceptions, the SCSPI is highest in the Western Bundeslaender, followed by the GCSPI and, finally the AROPR. In the Eastern Bundeslaender, the trend is just the opposite, i.e. the AROPR is highest, followed by the GCSPI and, finally, the SCSPI.

Third, the three indices do not only demonstrate rather different degrees of volatility over time, poverty dynamics can even be opposite. The SCSPI is rather volatile and tends to be higher in more populous, urbanised areas. It seems to be distorted as dissatisfaction in one dimension influences the evaluation of other dimensions, especially if respondents suffer from bad health conditions. The AROPR, on the other hand, is less volatile and tends to be higher in less populous, rural areas. By definition it is rather sensitive to the overall inequality in the society and, as a consequence, might decrease during a time in which the situation of the poor actually worsened, i.e. whenever the situation of the poor worsens less than the situation of the wealthy. The GCSPI, finally, is unable to reflect these changes in the overall inequality of the society; it only captures inequality changes among the poor.

The results all seem to point in one direction, i.e. that the introduction of multidimensional poverty indices that complement the AROPR seems to be very worthwhile as these indices provide information about poverty that traditional income-based poverty measures are unable to discover. In addition, the empirical analysis offered a series of interesting trends. For once, there is the worrisome trend towards a concentration of poverty in Eastern Germany. There are also the very interesting results of the development of poverty rates in the different Bundeslaender over time that highlight stories of success and failure. Also, the differences in the dimensional decompositions, according to regions and over time, provide lots of material to study. It seems that further research in this area could provide valuable insight. In addition, direct capability-related questions will for the first time ever be available in the GSOEP in 2013. 


\section{Bibliography}

Alkire, S., Santos, M.E. Acute multidimensional poverty: a new index for developing countries. OPHI Working Paper No. 38 (2010)

Alkire, S., Foster, J. Counting and multidimensional poverty measurement. OPHI Working Paper No. 7 (2007)

Alkire, S., Foster, J. Counting and multidimensional poverty measurement. J Public Econ 95, 476-487 (2011)

Anand, S., Kanbur, R. (1993). The Kuznets process and the inequality-development relationship. J Dev Econ 40, 25-52 (1993)

Aristondo, O., de la Vega, C.L., Urrutia, A. A new multiplicative decomposition for the Foster-GreerThorbecke poverty indices. B Econ Res 62, 259-267 (2010)

Arndt, C., Volkert, J. A capability approach for official German Poverty and Wealth reports: conceptual background and first empirical results. IAW Working Paper No. 27. Institute for Applied Economic Research, Tübingen (2007)

Arrow, K. A difficulty in the concept of social welfare. J Polit Econ 58, 328-346 (1950)

Atkinson, A., Bourguignon, F. The comparison of multi-dimensioned distributions of economic status. Rev Econ Stud 49, 183-201 (1982)

Bartlett, R. C., Collins, S. D. Aristotle's Nicomachean ethics: a new translation. The University of Chicago Press, Chicago (2011)

Bentham, J. An introduction to the principles of morals and legislation. Dover Publications, Inc., New York (2007)

Bérenger, V., Bresson, F. Axiomatic and robust multidimensional poverty measurements in five South Mediterranean countries. Paper presented at the 2010 HDCA Conference

Bergson, A. A reformulation of certain aspects of welfare economics. Q J Econ 52, 310-334 (1938)

Bern, C. et al. Assessment of potential indicators for protein-energy malnutrition in the algorithm for integrated management of childhood illness. B World Health Organ 75, 87-96 (1997) 
BMFSFJ. Monitor Familienleben 2012. BMFSFJ, Berlin (2012)

Böckler Impuls. Mindestlohn: die Linie nach unten. Böckler Impuls 11, 1 (2006)

Boland, P.J., Proschan, F.: Multivariate arrangement increasing functions with applications in probability and statistics. J Multivar Anal 25, 286-298 (1988)

Bossert, W., Chakravarty, S.R., D'Ambrosio, C. Multidimensional poverty and material deprivation. ECINEQ Working Paper No. 129 (2009)

Bossert, W., Chakravarty, S.R., D’Ambrosio, C. Multidimensional poverty and material deprivation with discrete data.

http://pages.videotron.com/wbossert/multidimensional_poverty_jun12.pdf (2012)

Bourguignon, F. The poverty-growth-inequality triangle. Indian Council for Research on International Economic Relations Working Paper No. 125 (2004)

Bourguignon, F. The growth elasticity of poverty reduction: explaining heterogeneity across countries and time-periods. In: Eichler, T., Turnovsky, S. (eds.) Growth and Inequality: Theory and Policy Implications, pp. 3-27. MIT Press, Cambridge, Mass. (2003)

Bourguignon, F., Chakravarty, S.R. A family of multidimensional poverty measures. In: Slottje, D. (ed.) Advances in Econometrics, Income Distribution and Scientific Methodology, pp. 331-344. Physica-Verlag, Heidelberg (1999)

Bourguignon, F., Chakravarty, S.R. The measurement of multidimensional poverty. J. Econ. Inequal. 1, 25-49 (2003)

Burmeister, K. Leiharbeit 2012: Zwischen gewerkschaftlichem Erfolg, unternehmerischen Ausweichmanövern und politischer Verantwortung. Friedrich-Ebert-Stiftung, Bonn (2012)

Chakravarty, S.R., D'Ambrosio, C. The measurement of social exclusion. Rev Income Wealth 52, 377-398 (2006)

Chakravarty, S.R., Mukherjee, D., Ranade, R.R. On the family of subgroup and factor decomposable measures of multidimensional poverty. Res Econ Inequal 8, 175-194 (1998)

Chakravarty, S.R., Silber, J. Measuring multidimensional poverty: the axiomatic approach. In: Kakwani, N., Silber, J. (eds.) Quantitative Approaches to Multidimensional Poverty Measurement, pp. 192-209. Palgrave Macmillan, New York (2008)

Coelli, T., Rao, D.S.P., Battese, G.E. An introduction to efficiency and productivity analysis. Kluwer Academic Publishers, Boston (1998) 
Cover, T. M., Thomas, J.A. Elements of Information Theory. John Wiley \& Sons Inc., Second Edition, New Jersey (2006)

Dasgupta, P., Ray, D. Inequality as a determinant of malnutrition and unemployment: theory. Econ J 96, 1011-1034 (1986)

De la Vega, C.L., Urrutia, A., de Sarachu, A. Characterizing multidimensional inequality measures which fulfil the Pigou-Dalton bundle principle. Soc Choice Welf 35, 319-329 (2010)

Deininger, K. Causes and consequences of civil strife: micro-level evidence from Uganda. Oxford Econ Pap 55, 579-606 (2003)

Deininger, K., Squire, L. New ways of looking at old issues: inequality and growth. J Dev Econ 57, 259-287 (1998)

Deutsch J., Silber, J. Measuring multidimensional poverty: an empirical comparison of various approaches. Rev Income Wealth 51, 145-174 (2005)

Deutscher Bundestag. Lebenslagen in Deutschland: Entwurf des 4. Armuts- und Reichtumsberichts des Bundesregierung. Stand 17.09.2012. http://www.sozialpolitik-aktuell.de/tl_files/sozialpolitikaktuell/_Politikfelder/Einkommen-Armut/Dokumente/Entwurf\%204.\%20Armutsbericht\%20der\%20 Bundesregierung\%2017.9.2012.pdf

Drèze, J., Sen, A. Hunger and Public Action. Oxford University Press, Oxford (1989)

Duclos, J-Y., Sahn, D.E., Younger, S.D. Robust multidimensional poverty comparisons. Econ J 116, 943-968 (2006)

Dworkin, R. What is equality? Part 1: equality of welfare. Philos Public Aff 10 (3), 185-246 (1981a)

Dworkin, R. What is equality? Part 2: equality of resources. Philos Public Aff 10 (4), 283-345 (1981b)

Educational Reporting Consortium. Education in Germany: An indicator-based report including an analysis of education and migration. (2012)

Faik, J. Income inequality and poverty in front of and during the economic crisis - an empirical investigation for Germany 2002-2010. ECINEQ Working Paper No. 255 (2012)

Fields, G. Distribution and development: a summary of the evidence for the developing world. Background paper prepared for the World Development Report 2000 (1999)

Figari, F. Cross-national differences in determinants of multiple deprivation in Europe. J Econ Inequal 10, 397-418 (2012) 
Foster, J. A class of chronic poverty measures. In: Addison, T., Hulme, D., Kanbur, R. (eds.) Poverty Dynamics: Interdisciplinary Perspectives, pp. 59-76. Oxford University Press, Oxford (2009)

Foster, J., Greer, J., Thorbecke, E. A class of decomposable poverty measures. Econometrica 52, 761$766(1984)$

Grimm, M., Harttgen, K., Klasen, S., Misselhorn, M., Munzi, T., Smeeding, T. Inequality in human development: an empirical assessment of 32 countries. Soc Indic Res 97, 191-211 (2010)

Harsanyi, J. Cardinal utility in welfare economics and in the theory of risk-taking. J Polit Econ 61, 434-435 (1953)

Jayaraj, D., Subramanian, S. A Chakravarty-D' Ambrosio view of multidimensional deprivation: some estimates for India. Econ Polit Wkly 45, 53-65 (2010)

Jenkins, S., Lambert, P. Three I's of poverty curves, with an analysis of UK poverty trends. Oxford Econ Papers 49, 317-327 (1997)

Kakwani, N., Silber, J. Introduction: on quantitative approaches to multidimensional poverty measurement In: Kakwani, N., Silber, J. (eds.) Quantitative Approaches to Multidimensional Poverty Measurement, pp. xviii-xxiv. Palgrave Macmillan, New York (2008)

Kanbur, R. Income distribution and development. In: Atkinson, A., Bourguignon, F. (eds.) Handvolume of Income Distribution, Vol. 1, pp. 791-841. Elsevier, Amsterdam (2000)

Kanbur, R., Lustig, N. Why is Inequality Back on the Agenda? Department of Agricultural, Resource and Managerial Economics Cornell University Working Paper No. 99-14 (1999)

Kemming, H., Borbach, C. Transport and social exclusion: a G7 comparison: an overview of the German position, Research Institute for Regional and Urban Development of the Federal State of North Rhine-Westphalia (2003)

Klasen, S. Measuring poverty and deprivation in South Africa. Rev Income Wealth 46, 33-58 (2000)

Kleinman, A., Eisenberg, L., Good, B. Culture, illness, and care: clinical lessons from anthropologic and cross-cultural research. Ann Intern Med 88, 251-258 (1978)

Kleinman, A. The illness narratives: suffering, healing, and the human condition. Basic Volumes, New York (1988)

Kolm, S.C. Multidimensional egalitarianisms. Q J Econ 91, 1-13 (1977) 
Lovell, C.A.K., Richardson, S. Travers, P. Wood, L. Resources and functionings: a new view of inequality in Australia. In: Eichhorn, W. (ed.) Models and Measurement of Welfare and Inequality, pp. 787-807. Springer, Heidelberg (1994)

Maasoumi, E. The measurement and decomposition of multidimensional inequality. Econometrica 54(4), 991-997 (1986)

Maasoumi, E., Lugo, M.A. The information basis of multivariate poverty assessments. In: Kakwani, N., Silber, J. (eds.) Quantitative Approaches to Multidimensional Poverty Measurement, pp. 1-29. Palgrave Macmillan, New York (2008)

Mack, J., Lindsay, S. Poor Britain. George Allen and Unwin Ltd., London (1985)

Marrero, G., Rodríguez, J. Inequality of opportunity and growth. ECINEQ Working Paper No. 154 (2010)

Merz, J. Time and economic well-being - a panel analysis of desired versus actual working hours. Rev Income Wealth 48, 317-346 (2002)

Muller, E.N., Seligson, M.A. Inequality and insurgency. Am Polit Sci Rev 81, 425-452 (1987)

Nussbaum, M. Capabilities as fundamental entitlements: Sen and social justice. Fem Econ 9, 33-59 (2003)

Pigou, A. 1932. The economics of welfare: Volume I, Band 1. Cosimo, Inc., New York (2005)

Qizilbash, M., Clark, D. The capability approach and fuzzy poverty measures: an application to the South African context. Soc Indic Res 74, 103-139 (2005)

Ravallion, M. Growth, inequality and poverty: looking beyond averages. World Dev 29 (11), 1803$1815(2001)$

Ravallion, M. Inequality is bad for the poor. In: Jenkins, S. P., John Micklewright, J. (eds.) Inequality and Poverty Re-Examined, pp. 37-62. Oxford University Press, Oxford (2007)

Rawls, J. A theory of justice. Harvard University Press, Cambridge (1971)

Rippin, N. The concept of multidimensional poverty: accounting for dimensional poverty. IAI Discussion Paper No. 179. University of Göttingen, Göttingen (2009)

Robbins, L. Interpersonal comparisons of utility: a comment. Econ J 48, 635-641 (1938)

Roemer, J. E. Theories of distributive justice. Havard University Press, Cambridge Massachusetts (1998)

Samuelson, P. Consumption theory in terms of revealed preference. Economica 15, 243-253 (1948) 
Sen, A. Poverty: an ordinal approach to measurement. Econometrica 44, 219-231 (1976)

Sen, A. Commodities and capabilities. Oxford University Press, Oxford (1985)

Sen, A. The standard of living. Cambridge University Press, Cambridge (1987)

Sen, A. Inequality Reexamined. Oxford University Press, Oxford (1992)

Sen, A. On economic inequality. Oxford University Press, Oxford (1997)

Sen, A. The idea of justice. Harvard University Press, Cambridge Massachusetts (2009)

Seth, S. A class of distribution and association sensitive multidimensional welfare indices. J Econ Inequal DOI 10.1007/s10888-011-9210-3 (2011)

Silber, J. A comment on the MPI index. J Econ Inequal 9, 479-481 (2011)

Smith, A. 1776. An Inquiry into the Nature and Causes of the Wealth of Nations, Volume II. Oxford: Clarendon Press (1869)

Sugden, R. Welfare, resources, and capabilities: a review of inequality reexamined by Amartya Sen. J Econ Lit 31(4), 1947-1962 (1993)

Tsui, K.Y. Multidimensional inequality and multidimensional generalized entropy measures: An axiomatic derivation. Soc Choice Welf 16, 145-157 (1999)

Tsui, K.Y. Multidimensional poverty indices. Soc Choice Welf 19, 69-93 (2002)

UNDP Human Development Report 1995. Oxford University Press, New York (1995)

Volkert, J. et al. Operationalisierung der Armuts- und Reichtumsmessung. In: Lebenslagen in Deutschland, Bundesministerim für Gesundheit und Soziale Sicherung, Bonn (2004)

Wagner, G., Frick, J., Schupp, J. The German Socio-Economic Panel study (SOEP) - scope, evolution and enhancements. German Institute for Economic Research, Berlin (2007)

White, H., Anderson, E. Growth versus distribution: does the pattern of growth matter? Dev Policy Rev 19 (3), 267-289 (2001)

Zadeh, L.A. Fuzzy Sets. Inform Control 8, 338-353 (1965) 


\section{Appendix 1}

\section{Proof of Theorem 1.1}

The 'if' part of the proposition is straightforward to verify. To prove the 'only if' part, I proceed by induction on population size (see also Seth, 2011; Bossert, Chakravarty and D'Ambrosio, 2012). Suppose that the new index $P(\mathbf{X} ; \mathbf{z})$ satisfies the axioms stated in the proposition. Since the scope of this paper is restricted to the ordinal case, assume without loss of generality that $\mathbf{X}$ is a dichotomous matrix such that $x_{i j} \in\{0,1\}$ for all $i \in\{1, \ldots, n\}$ and for all $j \in\{1, \ldots, k\}$. It immediately follows that individual $i$ is deprived with regard to attribute $j$ if $x_{i j}=0$, i.e. $x_{i j}=0 \Leftrightarrow c_{i j}=1$ and $x_{i j}=1 \Leftrightarrow c_{i j}=0$. Thus, $P(\mathbf{X} ; \mathbf{z})$ can be expressed as $P(\mathbf{X})$.

Now suppose $\mathbf{X} \in X_{1}$. Be $\mathbf{0}_{1}$ a vector of zeros and $\mathbf{1}_{1}$ a vector of ones. Then by normalization (NM), $P\left(\mathbf{0}_{1}\right)=1$ and $P\left(\mathbf{1}_{1}\right)=0$. These equations in combination with strong focus (SF) lead to the following general identification function:

$$
\rho_{C S}\left(\mathbf{c}_{1} ; \mathbf{z}\right)=\left\{\begin{array}{cll}
f\left(\mathbf{c}_{1 .}\right) & \text { if } & \max \left\{\mathbf{c}_{1 .}=1\right\} \\
0 & \text { if } & \max \left\{\mathbf{c}_{1 .}=0\right\}
\end{array}\right.
$$

Suppose $\mathbf{X} \in X_{1} \backslash\left\{\mathbf{1}_{1}\right\}$. Let $0_{j}$ denote deprivation in some attribute $j \in\{1, \ldots, k\}$ so that $P\left(0_{j}\right)=f\left(\mathbf{c}_{1}\right) a_{j}$. Aggregating under factor decomposability (FD) leads to:

$$
P(\mathbf{X})=\sum_{j \in\{1, \ldots, k\}: c_{1 j}=1} P\left(\mathbf{0}_{j}\right)=\sum_{j \in\{1, \ldots, k\} ; c_{1 j}=1} f\left(\mathbf{c}_{1}\right) a_{j}=f\left(\mathbf{c}_{1}\right) \sum_{j \in\{1, \ldots, k\}, c_{1 j}=1} a_{j} .
$$

From monotonicity (MN) and sensitivity to inequality increasing switch (SIIS) it follows that $f\left(\mathbf{c}_{1}\right.$ ) is increasing in $\mathbf{c}_{1}$. with a nondecreasing (nonincreasing) marginal in case attributes are substitutes (complements). From monotonicity (MN) and normalization (NM) it follows that $f\left(\mathbf{c}_{1 .}\right) \in(0,1]: \rho_{C S}\left(\mathbf{c}_{1} ; \mathbf{z}\right)=f\left(\mathbf{c}_{1}\right)$ only if $c_{1 j}=1$ for at least one $j \in\{1, \ldots, k\}$, so that due to monotonicity $(\mathrm{MN}) f\left(\mathbf{c}_{1}\right)>0$. Also, due to normalization $(\mathrm{NM})$ :

$$
P\left(\mathbf{0}_{1}\right)=f\left(\mathbf{c}_{1 .}\right) \sum_{j \in\{1, \ldots, k\} ; c_{1 j}=1} a_{j}=f\left(\mathbf{c}_{1}\right) \sum_{j=1}^{k} a_{j}=f\left(\mathbf{c}_{1 .}\right)=1 .
$$

Thus, $f\left(\mathbf{c}_{1 .}\right) \in(0,1]$.

Suppose proposition 1 is true for all $n \in \mathbf{N}$. Now, let

$$
\mathbf{X} \in X_{\mathrm{n}+1}, \mathbf{X}^{\prime}=\left\{\mathbf{x}_{i j} \mid i \in\{1, \ldots, n\}, j \in\{1, \ldots, k\}\right\} \text { and } \mathbf{X}^{\prime \prime}=\left\{\mathbf{x}_{i j} \mid i=n+1, j \in\{1, \ldots, k\}\right\}
$$


When extending $f\left(\mathbf{c}_{1}\right)$ to a society with $n$ individuals, the identification function in its most general form may $i$ ) depend on the deprivation vectors of other individuals, $i i$ ) differ across individuals, iii) depend on the population size $n$.

The first possibility is immediately ruled out by subgroup decomposability (SD), i.e. $f_{i}^{n}\left(\mathbf{c}_{i} \times\left\{\mathbf{c}_{1}, \ldots, \mathbf{c}_{i-1}, \mathbf{c}_{i+1}, \ldots, \mathbf{c}_{n}, \mathbf{c}_{n+1}\right\}\right)=f_{i}^{n}\left(\mathbf{c}_{i}\right)$ for all $i \in \mathbf{N}$. With this, I can rewrite (1.01) as:

$$
\begin{gathered}
P(\mathbf{X})=\frac{n}{n+1} P\left(\mathbf{X}^{\prime}\right)+\frac{1}{n+1} P\left(\mathbf{X}^{\prime \prime}\right) \Leftrightarrow \\
P(\mathbf{X})=\left(\frac{n}{n+1}\right)\left(\frac{1}{n}\right) \sum_{i=1}^{n} f_{i}^{n+1}\left(\mathbf{c}_{i \cdot}\right) \sum_{j \in\{1, \ldots, k\} c_{c j}=1} a_{j}+\left(\frac{1}{n+1}\right) f_{n+1}^{n+1}\left(\mathbf{c}_{n+1}\right) \sum_{j \in\{1, \ldots, k\} c_{n+1 j}=1} a_{j}
\end{gathered}
$$

Next, I will show that the second possibility can be excluded, i.e. $f_{i}^{n}=f_{i^{\prime}}^{n}$ for all $i, i^{\prime} \in \mathbf{N}$.

Consider any $\hat{i}, \tilde{i} \in \mathbf{N}$. Let $\mathbf{X} \in X_{\mathrm{n}}$ whereby $\mathbf{c}_{\hat{i}}=\overline{\mathbf{c}}$. with $\mathbf{0}_{\hat{\mathrm{i}}} \neq \overline{\mathbf{c}} \neq \mathbf{1}_{\hat{i}}$ and $\mathbf{c}_{i}=\mathbf{1}_{i}$ for all $i \neq \hat{i}$. Likewise, let $\mathbf{X}^{\prime} \in X_{\mathrm{n}}$ be such that $\mathbf{c}_{\tilde{i}}=\overline{\mathbf{c}}$ and $\mathbf{c}_{i .}=\mathbf{1}_{i}$ for all $i \neq \tilde{i}$. Using normalization (NM) and subgroup decomposition (SD):

$$
P(\mathbf{X})=(n-1) / n+f_{\hat{i}}^{n}(\overline{\mathbf{c}}) \sum_{j \in\{1, \ldots, k\} \bar{c}_{j}=1} a_{j} \text { and } P\left(\mathbf{X}^{\prime}\right)=(n-1) / n+f_{\tilde{i}}^{n}(\overline{\mathbf{c}}) \sum_{j \in\left\{1, \ldots, k, \bar{c}_{j}=1\right.} a_{j}
$$

From anonymity (AN) it follows that $P(\mathbf{X})=P\left(\mathbf{X}^{\prime}\right)$ and thus $f_{\hat{i}}^{n}(\overline{\mathbf{c}})=f_{\tilde{i}}^{n}(\overline{\mathbf{c}})$. Hence, $f_{i}^{n}=f_{i^{\prime}}^{n}$ for all $i, i^{\prime} \in \mathbf{N}$. I denote this common function $f^{n}$.

Finally, also the third possibility can be excluded, i.e. $f^{n}=f^{n^{\prime}}$ for all $n, n^{\prime} \in \mathbf{N}$ :

Consider any $\mathbf{X} \in X_{1}$ so that $\mathbf{c}_{1}=\overline{\mathbf{c}}$ is any deprivation vector resulting from the achievement matrix $\mathbf{X}$.

Thus, $P(\mathbf{X})=f^{1}(\overline{\mathbf{c}}) \sum_{j \in\{1, \ldots, k\}, c_{j}=1} a_{j}$.

Now, consider any $\overline{\mathbf{X}} \in X_{\mathrm{n}}$ so that $\overline{\mathbf{X}}=[\mathbf{X}]_{n}$. Then, by population principle (PP)

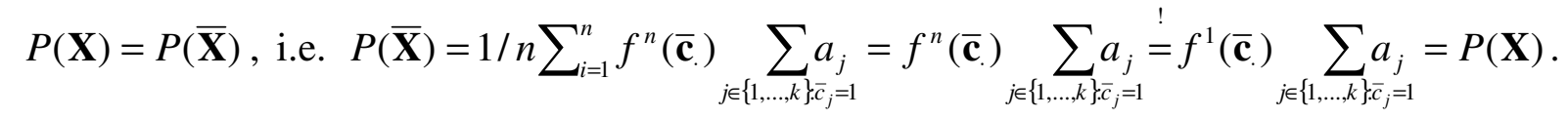

As a result, $f^{1}(\overline{\mathbf{c}})=f^{n}(\overline{\mathbf{c}})$ and thus $f^{n^{\prime}}=f^{n}$ for all $n, n^{\prime} \in \mathbf{N}$. I denote this common function $f$.

With this I can rewrite equation (1.02) as

$$
\begin{gathered}
P(\mathbf{X})=\left(\frac{n}{n+1}\right)\left(\frac{1}{n}\right) \sum_{i=1}^{n} f\left(\mathbf{c}_{i \cdot}\right) \sum_{j \in\{1, \ldots, k\} c_{c_{j j}=1} a_{j}}+\left(\frac{1}{n+1}\right) f\left(\mathbf{c}_{n+1}\right) \sum_{j \in\{1, \ldots, k\} c_{n+1}=1} a_{j} \Leftrightarrow \\
P(\mathbf{X})=\frac{1}{n+1} \sum_{i=1}^{n+1} f\left(\mathbf{c}_{i:}\right) \sum_{j \in\{1, \ldots, k\} c_{i j j}=1} a_{j}
\end{gathered}
$$

QED. 


\section{Proof of Proposition 1.1}

I proof proposition 1 for the new class of indices, the proof for the others is equivalent.

Define $d_{i}=\#\left\{c_{i j} \mid c_{i j}=1\right\}$ and further $R_{j} \equiv\left\{i \in \mathbf{N}\left\{d_{i}=j\right\}, j=1, \ldots, k\right\}$ with $\# R_{j}=q_{j} ; j=1, \ldots, k$ and $H_{j}=q_{j} / n$. Then

$$
P_{C S}(\mathbf{X} ; \mathbf{z})=1 / n \sum_{i \in S_{j}} \delta_{i}^{\alpha} \sum_{j \in\{1, \ldots, k\} c_{c_{i j}=1}} a_{j}=1 / n\left[\left(d_{1} / k\right)^{\alpha+1}+\ldots+\left(d_{n} / k\right)^{\alpha+1}\right]=\sum_{j=1}^{k}(j / k)^{\alpha+1} H_{j}
$$

QED.

\section{Proof of Proposition 1.3}

$$
\begin{aligned}
& P_{C S}(\mathbf{X} ; \mathbf{z})=\frac{1}{n} \sum_{i \in S_{j}} \delta_{i}^{\alpha} \sum_{j \in\{1, \ldots, k\}: c_{i j}=1} a_{j}=\frac{1}{n} \sum_{i \in S_{j}}\left[\sum_{j \in\{1, \ldots, k\}: c_{i j}=1} a_{j}\right]^{\alpha} \sum_{j \in\{1, \ldots, k\}: c_{i j}=1} a_{j}=\frac{1}{n} \sum_{i \in S_{j}}\left(\sum_{j \in\{1, \ldots, k\}: c_{i j}=1} a_{j}\right)^{\theta:=\alpha+1} \Leftrightarrow \\
& P_{C S}(\mathbf{X} ; \mathbf{z})=\left(\frac{q}{n}\right)\left(\frac{1}{q}\right)\left\{\sum_{i \in S_{j}}\left(\left[\frac{1}{q} \sum_{i \in S_{j}} \sum_{j \in\{1, \ldots, k\}: c_{i j}=1} a_{j}\right]^{\theta}\left[\sum_{j \in\{1, \ldots, k\}: c_{i j}=1} a_{j}\right]^{\theta} /\left[\frac{1}{q} \sum_{i \in S_{j}} \sum_{j \in\{1, \ldots, k\} ; c_{i j}=1} a_{j}\right]^{\theta}\right)\right\} \Leftrightarrow \\
& P_{C S}(\mathbf{X} ; \mathbf{z})=\frac{q}{n}\left\{\left[\frac{1}{q} \sum_{i \in S_{j}} \sum_{j \in\{1, \ldots, k\}\}_{c_{i j}=1}} a_{j}\right]^{\theta}\left[\frac{(\theta(\theta-1))}{q(\theta(\theta-1))}+1-1\right] \sum_{i \in S_{j}}\left[\sum_{j \in\{1, \ldots, k\} c_{c i j}=1} a_{j} / \frac{1}{q} \sum_{i \in S_{j}} \sum_{j \in\{1, \ldots, k\} ; c_{i j}=1} a_{j}\right]^{\theta}\right\} \Leftrightarrow \\
& P_{C S}(\mathbf{X} ; \mathbf{z})=\frac{q}{n}\left[\frac{1}{q} \sum_{i \in S_{j}} \sum_{j \in\{1, \ldots, k\} c_{c i j}=1} a_{j}\right]^{\theta}\left[1+(\theta(\theta-1))\left[\frac{1}{q(\theta(\theta-1))} \sum_{i \in S_{j}}\left[\left[\sum_{j \in\{1, \ldots, k\} ; c_{i j}=1} a_{j} / \frac{1}{q} \sum_{i \in S_{j}} \sum_{j \in\{1, \ldots, k\} ; c_{i j}=1} a_{j}\right]^{\theta}-1\right]\right]\right]
\end{aligned}
$$

Thus, $P_{C S}(\mathbf{X} ; \mathbf{z})=H[\mu(\boldsymbol{\delta})]^{\theta}\left[1+(\theta(\theta-1)) G E_{\theta}(\boldsymbol{\delta})\right]$

QED. 


\section{Appendix $2 \mathrm{~A}$}

\section{Proof of Proposition 2.1.}

The 'if' part of the proposition is straightforward to verify. To prove the 'only if' part, I proceed by induction on population size (see also Rippin, 2012a). Suppose that the new index $P(\mathbf{X} ; \mathbf{z})$ satisfies the axioms stated in the proposition.

Individual $i$ is deprived in attribute $j$ if $x_{i j}<z_{j}$, i.e. $c_{i j}=1$. Likewise, $c_{i j}=0$ if $x_{i j} \geq z_{j}$.

Now suppose $\mathbf{X} \in X_{1}$. Let $\underline{\mathbf{x}}_{1}$ denote a vector of achievements with $\underline{x}_{1 j}<z_{j}$ for all $j$ and $\underline{\underline{\mathbf{x}_{1}}}$ a vector with zero achievement in all attributes, i.e. $\underline{\underline{x_{1 j}}}=0$ for all $j$. Finally, let $\overline{\mathbf{x}}_{1}$ be a vector of achievements with $\bar{x}_{1 j} \geq z_{j}$ for all $j$. Then $\overline{\overline{\text { by }}}$ normalization $(\mathrm{NM}), P\left(\underline{\underline{\mathbf{x}_{1}}}\right)=1$ and $P\left(\overline{\mathbf{x}}_{1}\right)=0$. Let $f\left(\mathbf{c}_{1}\right) \in[0,1]$ denote the general identification function of the poor. From monotonicity (MN) and inequality sensitivity (IS) it follows that $f\left(\mathbf{c}_{1}\right)$ is increasing in $\mathbf{c}_{1}$. with a nondecreasing (nonincreasing) marginal in case attributes are substitutes (complements). Thus, $\max \left\{f\left(\mathbf{c}_{1}\right)\right\}=1$ for all $\mathbf{x}_{1} \in \underline{\mathbf{x}_{1}}$, expressing absolute poverty and $\min \left\{f\left(\mathbf{c}_{1}\right)\right\}=0$ for all $\mathbf{x}_{1} \in \overline{\mathbf{x}_{1}}$, identifying the case of no poverty.

Suppose $\mathbf{X} \in X_{1} \backslash\left\{\overline{\mathbf{x}}_{1}\right\}$. Then there exists at least one achievement level $\tilde{x}_{1 j} \in \mathbf{X}$ with $\tilde{x}_{i j} \leq z_{j}$ for some $j \in\{1, \ldots, k\}$. Then, $P\left(\tilde{x}_{1 j}\right)=f\left(\mathbf{c}_{1 .}\right) a_{j} g\left(\tilde{x}_{1 j} ; z_{j}\right)$.

Aggregating under factor decomposability (FD) leads to the general formula:

$$
P(\mathbf{X} ; \mathbf{z})=\sum_{j \in\{1, \ldots, k\} ; c_{j}=1} f\left(\mathbf{c}_{1 .}\right) a_{j} g\left(x_{1 j} ; z_{j}\right)=f\left(\mathbf{c}_{1 .}\right) \sum_{j \in\{1, \ldots, k\} ; c_{1 j}=1} a_{j} g\left(x_{1 j} ; z_{j}\right)
$$

where $a_{j}>0$ and $\sum_{j=1}^{k} a_{j}=1$. Due to scale invariance (SI), $g\left(x_{i j} ; z_{j}\right)=g\left(x_{i j} / z_{j}\right)$ for all $(\mathbf{X} ; \mathbf{z}) \in \mathbf{K} \times \mathbf{Z}$ so that I can rewrite $(2.01)$ as

$$
P(\mathbf{X} ; \mathbf{z})=f\left(\mathbf{c}_{1 .}\right) \sum_{j \in\{1, \ldots, k\} ; c_{1 j}=1} a_{j} g\left(x_{1 j} / z_{j}\right)
$$

with $g[0, \infty] \rightarrow R^{1}$ being continuous and non-increasing due to continuity (CN) and monotinicity (MN). Also, fulfilment of uniform majorization (UM) requires convexity of $g($. (see Chakravarty, Mukherjee and Ranade 1998, p. 184). Finally, due to normalization,

$$
P\left(\underline{\underline{\mathbf{x}_{1}}}\right)=\sum_{j=1}^{k} a_{j} g(0)=g(0) \sum_{j=1}^{k} a_{j}=g(0)=1 .
$$


In addition, strong focus (SF) implies that $g(t)=c$ for all $t \geq 1$ with $c<1$ being a constant. Please note that $P\left(\overline{\mathbf{x}_{1}}\right)=0$ as required by normalization (NM) is already satisfied by $\min \left\{f\left(\mathbf{c}_{1 .}\right)\right\}=0$ for all $\mathbf{x}_{1} \in \overline{\mathbf{x}_{1}}$.

Suppose proposition 1 is true for all $n \in \mathbf{N}$. Now, let:

$$
\mathbf{X} \in X_{\mathrm{n}+1}, \mathbf{X}^{\prime}=\left\{\mathbf{x}_{i j} \mid i \in\{1, \ldots, n\}, j \in\{1, \ldots, k\}\right\} \text { and } \mathbf{X}^{\prime \prime}=\left\{\mathbf{x}_{i j} \mid i=n+1, j \in\{1, \ldots, k\}\right\}
$$

When extending $f\left(\mathbf{c}_{1}\right.$. to a society with $n$ individuals, the identification function in its most general form may $i$ ) depend on the deprivation vectors of other individuals, $i$ ) differ across individuals, iii) depend on the population size $n$.

The first possibility is immediately ruled out by subgroup decomposability (SD), i.e. $f_{i}^{n}\left(\mathbf{c}_{i \cdot} \times\left\{\mathbf{c}_{1}, \ldots, \mathbf{c}_{i-1}, \mathbf{c}_{i+1}, \ldots, \mathbf{c}_{n}, \mathbf{c}_{n+1}\right\}\right)=f_{i}^{n}\left(\mathbf{c}_{i \cdot}\right)$ for all $i \in \mathbf{N}$.With this, I can rewrite (2.03) as:

$$
\begin{gathered}
P(\mathbf{X} ; \mathbf{z})=\frac{n}{n+1} P\left(\mathbf{X}^{\prime} ; \mathbf{z}\right)+\frac{1}{n+1} P\left(\mathbf{X}^{\prime \prime} ; \mathbf{z}\right) \Leftrightarrow \\
P(\mathbf{X} ; \mathbf{z})=\left(\frac{n}{n+1}\right)\left(\frac{1}{n}\right) \sum_{i=1}^{n} f_{i}^{n+1}\left(\mathbf{c}_{i:}\right) \sum_{j \in\{1, \ldots, k\} c_{i j}=1} a_{j} g\left(x_{i j} / z_{j}\right)+\left(\frac{1}{n+1}\right) f_{n+1}^{n+1}\left(\mathbf{c}_{n+1}\right) \sum_{j \in\{1, \ldots, k\} ; c_{n+1 j}=1} a_{j} g\left(x_{i j} / z_{j}\right)
\end{gathered}
$$

Next, I will show that the second possibility can be excluded, i.e. $f_{i}^{n}=f_{i^{\prime}}^{n}$ for all $i, i^{\prime} \in \mathbf{N}$.

Consider any $\hat{i}, \tilde{i} \in \mathbf{N}$. Let $\mathbf{X} \in X_{\mathrm{n}}$ whereby $\mathbf{x}_{\hat{i}}=\hat{\mathbf{x}}$ with $\underline{\mathbf{x}} \neq \hat{\mathbf{x}} \neq \overline{\mathbf{x}}$ and $\mathbf{x}_{i .}=\overline{\mathbf{x}}$ for all $i \neq \hat{i}$. Likewise, let $\mathbf{X}^{\prime} \in X_{\mathrm{n}}$ be such that $\mathbf{x}_{\tilde{i}}^{\prime}=\hat{\mathbf{x}}$ and $\mathbf{x}_{i}^{\prime}=\overline{\mathbf{x}}$ for all $i \neq \tilde{i}$. Using normalization (NM) and subgroup decomposition (SD):

$$
P(\mathbf{X} ; \mathbf{z})=(n-1) / n+f_{\hat{i}}^{n}(\hat{\mathbf{c}}) \sum_{j \in\{1, \ldots, k\} \hat{c}_{j}=1} a_{j} g\left(\hat{x}_{. j} / z_{j}\right)
$$

And

$$
P\left(\mathbf{X}^{\prime} ; \mathbf{z}\right)=(n-1) / n+f_{\tilde{i}}^{n}(\hat{\mathbf{c}}) \sum_{j \in\{1, \ldots, k\} \hat{f}_{j}=1} a_{j} g\left(\hat{x}_{. j} / z_{j}\right)
$$

From anonymity (AN) it follows that $P(\mathbf{X} ; \mathbf{z})=P\left(\mathbf{X}^{\prime} ; \mathbf{z}\right)$ and thus $f_{\hat{i}}^{n}(\hat{\mathbf{c}})=f_{\tilde{i}}^{n}(\hat{\mathbf{c}})$. Hence, $f_{i}^{n}=f_{i^{\prime}}^{n}$ for all $i, i^{\prime} \in \mathbf{N}$. I denote this common function $f^{n}$.

Finally, also the third possibility can be excluded, i.e. $f^{n}=f^{n^{\prime}}$ for all $n, n^{\prime} \in \mathbf{N}$.

Consider any $\mathbf{X} \in X_{1}$ so that $\mathbf{x}_{1}=\hat{\mathbf{x}}$. is any achievements vector in $\mathbf{X}$. Thus,

$$
P(\mathbf{X} ; \mathbf{z})=f^{1}(\hat{\mathbf{c}} .) \sum_{j \in\{1, \ldots, k\} \hat{c}_{j}=1} a_{j} g\left(\hat{x}_{. j} / z_{j}\right)
$$

Now, consider any $\hat{\mathbf{X}} \in X_{\mathrm{n}}$ so that $\hat{\mathbf{X}}=[\mathbf{X}]_{n}$ and $\mathbf{z} \in \mathbf{Z}=\hat{\mathbf{z}} \in \mathbf{Z}$. Then, by population principle (PP) $P(\mathbf{X} ; \mathbf{z})=P(\hat{\mathbf{X}} ; \mathbf{z})$, i.e. 


$$
\begin{gathered}
P(\hat{\mathbf{X}} ; \mathbf{z})=1 / n \sum_{i=1}^{n} f^{n}(\hat{\mathbf{c}}) \sum_{j \in\{1, \ldots, k\} ; \hat{j}_{j}=1} a_{j} g\left(\hat{x}_{. j} / z_{j}\right)=f^{n}(\hat{\mathbf{c}}) \sum_{j \in\{1, \ldots, k\}, \hat{c}_{j}=1} a_{j} g\left(\hat{x}_{. j} / z_{j}\right) \stackrel{!}{=} \\
f^{1}(\hat{\mathbf{c}}) \sum_{j \in\{1, \ldots, k\} \hat{c}_{j}=1} a_{j} g\left(\hat{x}_{. j} / z_{j}\right)=P(\mathbf{X} ; \mathbf{z})
\end{gathered}
$$

As a result, $f^{1}(\hat{\mathbf{c}})=f^{n}(\hat{\mathbf{c}})$ and thus $f^{n^{\prime}}=f^{n}$ for all $n, n^{\prime} \in \mathbf{N}$. I denote this common function $f$.With this I can rewrite equation (2.04) as

$$
\begin{aligned}
& P(\mathbf{X} ; \mathbf{z})=\left(\frac{n}{n+1}\right)\left(\frac{1}{n}\right) \sum_{i=1}^{n} f\left(\mathbf{c}_{i}\right) \sum_{j \in\{1, \ldots, k\}, c_{i j}=1} a_{j} g\left(x_{i j} / z_{j}\right)+\left(\frac{1}{n+1}\right) f\left(\mathbf{c}_{n+1}\right) \sum_{j \in\{1, \ldots, k\} c_{n+1}=1} a_{j} g\left(x_{n+1 j} / z_{j}\right) \Leftrightarrow \\
& P(\mathbf{X} ; \mathbf{z})=\frac{1}{n+1} \sum_{i=1}^{n+1} f\left(\mathbf{c}_{i: .}\right) \sum_{j \in\{1, \ldots, k\} \cdot c_{i j}=1} a_{i} g\left(x_{i j} / z_{j}\right)
\end{aligned}
$$

QED.

Proof of Proposition 2.2

$$
\begin{aligned}
& P_{F G T}(\mathbf{X} ; \mathbf{z})=1 / n \sum_{i \in S_{j}} \sum_{j \in\{1, \ldots, k\}: c_{i j}=1} a_{j} g_{i j}^{\theta} \Leftrightarrow \\
& P_{F G T}(\mathbf{X} ; \mathbf{z})=1 / n \sum_{j \in\{1, \ldots, k\}, c_{i j}=1} a_{j} \sum_{i \in S_{j}}\left[g_{i j}\left(\left(z_{j}-\mu_{j}(\mathbf{g})\right) / z_{j}\right)\left(z_{j} /\left(z_{j}-\mu_{j}(\mathbf{g})\right)\right)\right]^{\theta} \Leftrightarrow \\
& P_{F G T}(\mathbf{X} ; \mathbf{z})=1 / n \sum_{j \in\{1, \ldots, k\} ; c_{i j}=1} a_{j}\left[\left(z_{j}-\mu_{j}(\mathbf{g})\right) / z_{j}\right]^{\theta} \sum_{i \in S_{j}}\left[g_{i j}\left(z_{j} /\left(z_{j}-\mu_{j}(\mathbf{g})\right)\right)\right]^{\theta} \Leftrightarrow \\
& \left.P_{F G T}(\mathbf{X} ; \mathbf{z})=1 / n \sum_{j \in\{1, \ldots, k\} c_{i j}=1} a_{j} \mid 1 / z_{j}\left(1 / q_{j} \sum_{i \in S_{j}} z_{j}-1 / q_{j} \sum_{i \in S_{j}} x_{i j}\right)\right]^{\theta} . \\
& \left.\sum_{i \in S_{j}} \mid g_{i j} /\left(1 / z_{j}\left|1 / q_{j} \sum_{i \in S_{j}} z_{j}-1 / q_{j} \sum_{i \in S_{j}} x_{i j}\right|\right)\right]^{\theta} \Leftrightarrow \\
& \left.P_{F G T}(\mathbf{X} ; \mathbf{z})=1 / n \sum_{j \in\{1, \ldots, k\} c_{i j}=1} a_{j} \mid 1 / q_{j} \sum_{i \in S_{j}} g_{i j}\right]_{i \in S_{j}}^{\theta}\left\lfloor g_{i j} /\left(1 / q_{j} \sum_{i \in S_{j}} g_{i j}\right)\right]^{\theta} \Leftrightarrow \\
& P_{F G T}(\mathbf{X} ; \mathbf{z})=q / n \sum_{j \in\{1, \ldots, k\} c_{c i j}=1} a_{j}(1 / q)\left[\mu_{j}(\mathbf{g})\right]^{\theta} q_{j}\left[1+\left(\theta^{2}-\theta\right)\left(1 / q_{j}\left(\theta^{2}-\theta\right)\right) \sum_{i \in S_{j}}\left[g_{i j} / \mu_{j}(\mathbf{g})\right]^{\theta}-1 \rrbracket\right. \\
& \text { Thus, } P_{F G T}(\mathbf{X} ; \mathbf{z})=H \sum_{j \in\{1, \ldots, k\} ; c_{i j}=1} a_{j}\left(q_{j} / q\right)\left[\mu_{j}(\mathbf{g})\right]^{\theta}\left[1+\left(\theta^{2}-\theta\right) G E_{\theta}(\mathbf{g})\right]
\end{aligned}
$$

QED.

\section{Proof of Proposition 2.3.}

$$
\begin{gathered}
P_{I S}(\mathbf{X} ; \mathbf{z})=1 / n \sum_{i \in S_{j}} \sum_{j \in\{1, \ldots, k\} ; c_{i j}=1} a_{j} \delta_{i}^{\alpha} g_{i j}^{\theta} \Leftrightarrow \\
\left.P_{I S}(\mathbf{X} ; \mathbf{z})=1 / n \sum_{j \in\{1, \ldots, k\} c_{i j}=1} a_{j} \sum_{i \in S_{j}} \delta_{i}^{\alpha}\left|\sum_{i \in S_{j}}\left(\delta_{i} / \mu(\boldsymbol{\delta})\right)^{\alpha}\right| \mathbb{1} / \sum_{i \in S_{j}}\left(\delta_{i} / \mu(\boldsymbol{\delta})\right)^{\alpha}\right] .
\end{gathered}
$$


APPENDIX 2 A

109

$$
\begin{aligned}
& \left.g_{i j}^{\theta} \mid \sum_{i \in S_{j}}\left(g_{i j} / \mu_{j}(\mathbf{g})\right)^{\theta} \mathbb{1} / \sum_{i \in S_{j}}\left(g_{i j} / \mu_{j}(\mathbf{g})\right)^{\theta}\right] \Leftrightarrow \\
& \left.P_{I S}(\mathbf{X} ; \mathbf{z})=q / n \sum_{j \in\{1, \ldots, k\} ; c_{i j}=1} a_{j}\left(q_{j} / q\right) \sum_{i \in S_{j}}\left(1 / q_{j}\right) \delta_{i}^{\alpha} g_{i j}^{\theta} \mid \mathbb{l} / \sum_{i \in S_{j}}\left(\delta_{i} / \mu(\boldsymbol{\delta})\right)^{\alpha} \| \mathbb{1} / \sum_{i \in S_{j}}\left(g_{i j} / \mu_{j}(\mathbf{g})\right)^{\theta}\right] \text {. } \\
& \left\lfloor\sum_{i \in S_{j}}\left(g_{i j} / \mu_{j}(\mathbf{g})\right)^{\theta} \| \sum_{i \in S_{j}}\left(\delta_{i} / \mu(\boldsymbol{\delta})\right)^{\alpha}\right\rfloor \Leftrightarrow \\
& P_{I S}(\mathbf{X} ; \mathbf{z})=q / n \sum_{j \in\{1, \ldots, k\}: c_{i j}=1} a_{j}\left(q_{j} / q\right)[\mu(\boldsymbol{\delta})]^{\alpha}\left[\mu_{j}(\mathbf{g})\right]^{\theta}\left(1 / q_{j} \sum_{i \in S_{j}} \delta_{i}^{\alpha} g_{i j}^{\theta} /\left(1 / q \sum_{i \in S_{j}} \delta_{i}^{\alpha}\right)\left(1 / q_{j} \sum_{i \in S_{j}} g_{i j}^{\theta}\right) \|\right) . \\
& \left.\left.\left[1+\left(\theta^{2}-\theta\right) / q_{j}\left(\theta^{2}-\theta\right)\right]\right] \sum_{i \in S_{j}}\left[g_{i j} / \mu_{j}(\mathbf{g})\right]^{\theta}-1\right] . \\
& \left.\left[1+\left(\alpha^{2}-\alpha\right) / q\left(\alpha^{2}-\alpha\right)\right] \mid \sum_{i \in S_{j}}\left[\delta_{i} / \mu(\boldsymbol{\delta})\right]^{\alpha}-1\right]
\end{aligned}
$$

Thus,

$$
P_{I S}(\mathbf{X} ; \mathbf{z})=H \sum_{j \in\{1, \ldots, k\} ; c_{i j}=1} a_{j}\left(q_{j} / q\right)[\mu(\boldsymbol{\delta})]^{\alpha}\left[\mu_{j}(\mathbf{g})\right]^{\theta} I(\mathbf{g}, \boldsymbol{\delta})\left(1+\left(\theta^{2}-\theta\right) G E_{\theta}(\mathbf{g})\right)\left(1+\left(\alpha^{2}-\alpha\right) G E_{\alpha}(\mathbf{\delta})\right)
$$

QED. 
Appendix 2 B

TABLES 
Table 2.02: Decomposition of FGT and ISPI $\alpha=1.5$ (alphabetical ordering)

\begin{tabular}{|c|c|c|c|c|c|c|c|c|c|c|c|c|c|c|c|c|c|c|c|c|c|c|c|c|c|c|c|c|}
\hline \multirow[b]{3}{*}{ Country } & \multicolumn{18}{|c|}{ FGT $(\alpha=1.5)$} & \multicolumn{10}{|c|}{ ISPI $(\alpha=1.5)$} \\
\hline & \multirow[b]{2}{*}{$\Delta$} & \multirow[b]{2}{*}{ FGT } & \multirow[b]{2}{*}{$\mathrm{H}$} & \multicolumn{5}{|l|}{$\sigma$} & \multicolumn{5}{|l|}{$\mu(\mathrm{g})$} & \multicolumn{5}{|l|}{$\mathrm{GE}(\mathrm{g})$} & \multirow[b]{2}{*}{$\mu(\mathrm{d})$} & \multirow[b]{2}{*}{$\mathrm{GE}(\mathrm{d})$} & \multicolumn{5}{|l|}{$\mathrm{I}(\mathrm{g}, \mathrm{d})$} & \multirow[b]{2}{*}{ ISPI } & \multirow[b]{2}{*}{$\Delta$} & \multirow[b]{2}{*}{ Country } \\
\hline & & & & $\mathrm{g}_{1}$ & $\mathrm{~g}_{2}$ & $\mathrm{~g}_{3}$ & $\mathrm{~g}_{4}$ & $\mathrm{~g}_{5}$ & $\mathrm{~g}_{1}$ & $\mathrm{~g}_{2}$ & $\mathrm{~g}_{3}$ & $\mathrm{~g}_{4}$ & $\mathrm{~g}_{5}$ & $\mathrm{~g}_{1}$ & $\mathrm{~g}_{2}$ & $\mathrm{~g}_{3}$ & $\mathrm{~g}_{4}$ & $\mathrm{~g}_{5}$ & & & $\mathrm{~g}_{1}$ & $\mathrm{~g}_{2}$ & $\mathrm{~g}_{3}$ & $\mathrm{~g}_{4}$ & $\mathrm{~g}_{5}$ & & & \\
\hline Armenia & +1 & 0.009 & 0.246 & 0.24 & 0.05 & 0.02 & 0.46 & 0.37 & 0.245 & 0.298 & 0.444 & 0.253 & 0.491 & 0.352 & 0.378 & 0.205 & 0.229 & 0.104 & $\overline{0.203}$ & 0.145 & $\overline{0.960}$ & 1.005 & 2.109 & 1.637 & 0.686 & 0.001 & +1 & Armenia \\
\hline Azerbaijan & - & 0.016 & 0.360 & 0.17 & 0.10 & 0.02 & 0.66 & 0.33 & 0.248 & 0.327 & 0.538 & 0.262 & 0.440 & 0.340 & 0.331 & 0.182 & 0.174 & 0.160 & 0.250 & 0.107 & 1.157 & 1.436 & 1.896 & 1.374 & 0.888 & 0.003 & - & Azerbaijan \\
\hline Bangladesh & +4 & 0.117 & 0.829 & 0.35 & 0.27 & 0.30 & 0.63 & 0.79 & 0.310 & 0.319 & 0.574 & 0.350 & 0.748 & 0.270 & 0.311 & 0.145 & 0.142 & 0.099 & 0.424 & 0.178 & 1.369 & 1.655 & 1.857 & 1.469 & 1.220 & 0.057 & +2 & Bangladesh \\
\hline Benin & -2 & 0.147 & 0.841 & 0.13 & 0.24 & 0.52 & 0.66 & 0.60 & 0.246 & 0.359 & 0.687 & 0.335 & 0.459 & 0.342 & 0.356 & 0.112 & 0.158 & 0.355 & 0.452 & 0.121 & 1.462 & 1.562 & 1.424 & 1.255 & 1.078 & 0.066 & -2 & Benin \\
\hline Bolivia & -1 & 0.063 & 0.663 & 0.03 & 0.06 & 0.16 & 0.77 & 0.70 & 0.188 & 0.325 & 0.472 & 0.398 & 0.404 & 0.479 & 0.397 & 0.165 & 0.133 & 0.396 & 0.322 & 0.128 & 1.033 & 2.016 & 1.959 & 1.245 & 1.295 & 0.018 & -1 & Bolivia \\
\hline Cambodia & +1 & 0.140 & 0.927 & 0.24 & 0.15 & 0.28 & 0.92 & 0.44 & 0.260 & 0.302 & 0.462 & 0.508 & 0.483 & 0.321 & 0.332 & 0.169 & 0.070 & 0.286 & 0.436 & 0.097 & 1.424 & 1.924 & 1.765 & 1.053 & 1.396 & 0.055 & +5 & Cambodia \\
\hline Cameroon & - & 0.097 & 0.785 & 0.10 & 0.16 & 0.32 & 0.47 & 0.85 & 0.306 & 0.355 & 0.661 & 0.301 & 0.626 & 0.310 & 0.311 & 0.118 & 0.175 & 0.166 & 0.328 & 0.262 & 1.901 & 2.159 & 1.946 & 1.685 & 0.998 & 0.037 & -1 & ameroon \\
\hline Congo, Rep. & - & 0.075 & 0.824 & 0.21 & 0.14 & 0.08 & 0.67 & 0.79 & 0.303 & 0.329 & 0.538 & 0.319 & 0.717 & 0.299 & 0.343 & 0.162 & 0.166 & 0.100 & 0.314 & 0.163 & 1.505 & 2.011 & 2.098 & 1.416 & 1.127 & 0.021 & +1 & Zongo, Rep. \\
\hline DR Congo & - & 0.114 & 0.916 & 0.22 & 0.24 & 0.17 & 0.70 & 0.84 & 0.279 & 0.412 & 0.563 & 0.371 & 0.679 & 0.325 & 0.310 & 0.153 & 0.136 & 0.162 & 0.374 & 0.151 & 1.557 & 1.706 & 1.835 & 1.311 & 1.105 & 0.042 & - & DR Congo \\
\hline Ethiopia & - & 0.305 & 0.982 & 0.24 & 0.25 & 0.63 & 0.91 & 0.98 & 0.289 & 0.409 & 0.724 & 0.537 & 0.869 & 0.342 & 0.310 & 0.095 & 0.069 & 0.030 & 0.602 & 0.068 & 1.279 & 1.442 & 1.262 & 1.087 & 1.056 & 0.177 & - & Ethiopia \\
\hline Ghana & -3 & 0.076 & 0.711 & 0.06 & 0.06 & 0.23 & 0.70 & 0.60 & 0.241 & 0.306 & 0.666 & 0.335 & 0.543 & 0.300 & 0.433 & 0.115 & 0.158 & 0.231 & 0.325 & 0.144 & 1.671 & 1.882 & 1.882 & 1.210 & 1.095 & 0.023 & -2 & Ghana \\
\hline Haiti & -3 & 0.146 & 0.883 & 0.19 & 0.11 & 0.40 & 0.67 & 0.85 & 0.317 & 0.428 & 0.635 & 0.385 & 0.683 & 0.329 & 0.320 & 0.116 & 0.132 & 0.141 & 0.419 & 0.151 & 1.616 & 1.850 & 1.572 & 1.386 & 1.200 & 0.064 & -3 & Haiti \\
\hline India & +4 & 0.132 & 0.846 & 0.47 & 0.26 & 0.22 & 0.74 & 0.68 & 0.356 & 0.423 & 0.679 & 0.407 & 0.582 & 0.244 & 0.283 & 0.122 & 0.121 & 0.186 & 0.440 & 0.135 & 1.264 & 1.737 & 1.910 & 1.302 & 1.247 & 0.064 & +2 & ndia \\
\hline Kenya & - & 0.102 & 0.887 & 0.17 & 0.14 & 0.14 & 0.71 & 0.91 & 0.276 & 0.344 & 0.635 & 0.399 & 0.529 & 0.322 & 0.373 & 0.144 & 0.133 & 0.260 & 0.347 & 0.183 & 1.874 & 2.097 & 2.368 & 1.452 & 1.213 & 0.040 & - & Kenya \\
\hline Liberia & -7 & 0.150 & 0.904 & 0.14 & 0.18 & 0.33 & 0.69 & 0.79 & 0.238 & 0.377 & 0.734 & 0.370 & 0.807 & 0.335 & 0.335 & 0.098 & 0.133 & 0.046 & 0.398 & 0.144 & 1.498 & 1.518 & 1.711 & 1.244 & 1.135 & 0.061 & -2 & Liberia \\
\hline Malawi & +1 & 0.120 & 0.951 & 0.09 & 0.16 & 0.30 & 0.69 & 0.97 & 0.257 & 0.344 & 0.528 & 0.384 & 0.498 & 0.374 & 0.398 & 0.139 & 0.131 & 0.334 & 0.389 & 0.173 & 1.698 & 1.762 & 1.840 & 1.368 & 1.123 & 0.050 & +2 & Malawi \\
\hline Mali & - & 0.228 & 0.909 & 0.19 & 0.32 & 0.68 & 0.67 & 0.62 & 0.279 & 0.434 & 0.797 & 0.357 & 0.461 & 0.327 & 0.291 & 0.076 & 0.144 & 0.336 & 0.531 & 0.093 & 1.321 & 1.402 & 1.242 & 1.261 & 1.161 & 0.118 & - & Mali \\
\hline Moldova & -1 & 0.009 & 0.228 & 0.21 & 0.03 & 0.16 & 0.20 & 0.59 & 0.234 & 0.175 & 0.391 & 0.240 & 0.466 & 0.342 & 0.486 & 0.259 & 0.238 & 0.303 & 0.181 & 0.246 & 1.002 & 0.920 & 2.491 & 1.996 & 0.992 & 0.001 & -1 & Moldova \\
\hline Morocco & +2 & 0.070 & 0.578 & 0.20 & 0.10 & 0.45 & 0.55 & 0.50 & 0.250 & 0.369 & 0.578 & 0.278 & 0.528 & 0.298 & 0.366 & 0.164 & 0.202 & 0.198 & 0.381 & 0.139 & 1.067 & 1.763 & 1.502 & 1.424 & 1.312 & 0.027 & - & Morocco \\
\hline Mozambique & & 0.161 & 0.938 & 0.10 & 0.19 & 0.60 & 0.61 & 0.96 & 0.205 & 0.382 & 0.573 & 0.334 & 0.555 & 0.388 & 0.375 & 0.146 & 0.157 & 0.269 & 0.472 & 0.141 & 1.403 & 1.666 & 1.369 & 1.422 & 1.075 & 0.078 & - & Mozambique \\
\hline Namibia & +3 & 0.059 & 0.632 & 0.33 & 0.18 & 0.14 & 0.53 & 0.60 & 0.314 & 0.309 & 0.584 & 0.334 & 0.670 & 0.313 & 0.426 & 0.153 & 0.189 & 0.104 & 0.310 & 0.188 & 1.330 & 2.022 & 2.243 & 1.599 & 1.196 & 0.019 & +1 & Namibia \\
\hline Nepal & +4 & 0.138 & 0.903 & 0.34 & 0.29 & 0.34 & 0.64 & 0.86 & 0.309 & 0.343 & 0.629 & 0.375 & 0.558 & 0.278 & 0.300 & 0.129 & 0.142 & 0.203 & 0.443 & 0.178 & 1.455 & 1.781 & 1.744 & 1.448 & 1.161 & 0.072 & - & Nepal \\
\hline Niger & - & 0.296 & 0.971 & 0.21 & 0.40 & 0.68 & 0.77 & 0.90 & 0.271 & 0.460 & 0.844 & 0.387 & 0.739 & 0.287 & 0.254 & 0.054 & 0.131 & 0.077 & 0.595 & 0.079 & 1.364 & 1.324 & 1.220 & 1.173 & 1.054 & 0174 & - & Niger \\
\hline Nigeria & -2 & 0.131 & 0.836 & 0.21 & 0.29 & 0.30 & 0.60 & 0.75 & 0.270 & 0.468 & 0.823 & 0.333 & 0.461 & 0.331 & 0.291 & 0.061 & 0.152 & 0.350 & 0.394 & 0.190 & 1.559 & 1.658 & 1.922 & 1.439 & 1.180 & 0.062 & -4 & Nigeria \\
\hline Peru & - & 0.049 & 0.584 & 0.03 & 0.04 & 0.13 & 0.64 & 0.83 & 0.176 & 0.205 & 0.567 & 0.373 & 0.456 & 0.301 & 0.478 & 0.144 & 0.135 & 0.304 & 0.277 & 0.178 & 0.841 & 2.233 & 2.038 & 1.397 & 1.248 & 0.012 & - & Peru \\
\hline Swaziland & - & 0.053 & 0.641 & 0.08 & 0.08 & 0.12 & 0.70 & 0.55 & 0.199 & 0.331 & 0.579 & 0.330 & 0.643 & 0.292 & 0.506 & 0.168 & 0.159 & 0.111 & 0.292 & 0.137 & 1.634 & 1.462 & 2.032 & 1.295 & 1.064 & 0.013 & - & Swaziland \\
\hline Zambia & - & 0.100 & 0.839 & 0.12 & 0.16 & 0.16 & 0.83 & 0.79 & 0.264 & 0.285 & 0.490 & 0.422 & 0.524 & 0.360 & 0.402 & 0.183 & 0.125 & 0.297 & 0.376 & 0.118 & 1.533 & 1.673 & 2.127 & 1.196 & 1.162 & 0.035 & +1 & Zambia \\
\hline Zimbabwe & -1 & 0.066 & 0.799 & 0.13 & 0.11 & 0.04 & 0.59 & 0.92 & 0.261 & 0.307 & 0.523 & 0.338 & 0.703 & 0.374 & 0.392 & 0.166 & 0.147 & 0.150 & 0.269 & 0.215 & 1.983 & 2.044 & 2.378 & 1.559 & 1.095 & 0.016 & +1 & Zimbabwe \\
\hline
\end{tabular}


FGT $(\alpha=2)$ ISPI $(\alpha=2)$

\begin{tabular}{|c|c|c|c|c|c|c|c|c|c|c|c|c|c|c|c|c|c|c|c|c|c|c|c|c|c|c|c|c|}
\hline \multirow[b]{2}{*}{ Country } & \multirow[b]{2}{*}{$\Delta$} & \multirow[b]{2}{*}{ FGT } & \multirow[b]{2}{*}{$\mathrm{H}$} & \multicolumn{5}{|c|}{$\sigma=q_{j} / q$} & \multicolumn{5}{|l|}{$\mu(\mathrm{g})$} & \multicolumn{5}{|l|}{$\mathrm{GE}(\mathrm{g})$} & \multirow[b]{2}{*}{$\mu(\mathrm{d})$} & \multirow[b]{2}{*}{$\mathrm{GE}(\mathrm{d})$} & \multicolumn{5}{|l|}{$\mathrm{I}(\mathrm{g}, \mathrm{d})$} & \multirow[b]{2}{*}{ ISPI } & \multirow[b]{2}{*}{$\Delta$} & \multirow[b]{2}{*}{ Country } \\
\hline & & & & $\mathrm{g}_{1}$ & $\mathrm{~g}_{2}$ & $\mathrm{~g}_{3}$ & $\mathrm{~g}_{4}$ & $\mathrm{~g}_{5}$ & $\mathrm{~g}_{1}$ & $\mathrm{~g}_{2}$ & $\mathrm{~g}_{3}$ & $\mathrm{~g}_{4}$ & $\mathrm{~g}_{5}$ & $\mathrm{~g}_{1}$ & $\mathrm{~g}_{2}$ & $\mathrm{~g}_{3}$ & $\mathrm{~g}_{4}$ & $\mathrm{~g}_{5}$ & & & $\mathrm{~g}_{1}$ & $\mathrm{~g}_{2}$ & $\mathrm{~g}_{3}$ & $\mathrm{~g}_{4}$ & $\mathrm{~g}_{5}$ & & & \\
\hline Armenia & +1 & 0.006 & 0.246 & $\overline{0.24}$ & 0.05 & 0.02 & 0.46 & 0.37 & 0.245 & 0.298 & 0.444 & 0.253 & 0.491 & 0.373 & 0.423 & 0.217 & 0.247 & 0.107 & 0.203 & 0.140 & 0.941 & 0.898 & 2.442 & 1.761 & 0.737 & 0.000 & +1 & Armenia \\
\hline Azerbaijan & - & 0.011 & 0.360 & 0.17 & 0.10 & 0.02 & 0.66 & 0.33 & 0.248 & 0.327 & 0.538 & 0.262 & 0.440 & 0.369 & 0.353 & 0.181 & 0.179 & 0.162 & 0.250 & 0.102 & 1.337 & 1.698 & 2.199 & 1.475 & 0.916 & 0.001 & - & Azerbaijan \\
\hline Bangladesh & +4 & 0.096 & 0.829 & 0.35 & 0.27 & 0.30 & 0.63 & 0.79 & 0.310 & 0.319 & 0.574 & 0.350 & 0.748 & 0.278 & 0.330 & 0.143 & 0.138 & 0.090 & 0.424 & 0.176 & 1.455 & 1.879 & 2.061 & 1.590 & 1.297 & 0.040 & +2 & Bangladesh \\
\hline Benin & -2 & 0.126 & 0.841 & 13 & 0.24 & 0.52 & 0.66 & 0.60 & 0.246 & 0.359 & 0.687 & 0.335 & 0.459 & 368 & 0.378 & 0.106 & 0.156 & 0.338 & 0.452 & 0.118 & 1.632 & 1.775 & 1.515 & 1.318 & 1.107 & 0.047 & -2 & Benin \\
\hline Bolivia & -1 & 0.048 & 0.663 & .03 & 0.06 & 0.16 & 0.77 & 0.70 & 0.188 & 0.325 & 0.472 & 0.398 & 0.404 & 0.573 & 0.431 & 0.172 & 0.129 & 0.386 & 0.322 & 0.126 & 1.014 & 2.510 & 2.190 & 1.302 & 1.441 & 0.010 & -1 & olivia \\
\hline Cambodia & +3 & 0.110 & 0.927 & 0.24 & 0.15 & 0.28 & 0.92 & 0.44 & 0.260 & 0.302 & 0.462 & 0.508 & 0.483 & 0.337 & 0.359 & 0.176 & 0.066 & 0.276 & 0.436 & 0.100 & 1.580 & 2.370 & 1.984 & 1.067 & 1.541 & 0.034 & +5 & ambodia \\
\hline Cameroon & -1 & 0.083 & 0.785 & 0.10 & 0.16 & 0.32 & 0.47 & 0.85 & 0.306 & 0.355 & 0.661 & 0.301 & 0.626 & 0.333 & 0.325 & 0.113 & 0.176 & 0.150 & 0.328 & 0.262 & 2.102 & 2.486 & 2.112 & 1.816 & 1.008 & 0.025 & -1 & Cameroon \\
\hline Congo, Rep. & - & 0.059 & 0.824 & 0.21 & 0.14 & 0.08 & 0.67 & 0.79 & 0.303 & 0.329 & 0.538 & 0.319 & 0.717 & 0.316 & 0.370 & 0.164 & 0.166 & 0.090 & 0.314 & 0.159 & 1.606 & 2.364 & 2.424 & 1.526 & 1.190 & 0.012 & +2 & Congo, Rep. \\
\hline DR Congo & - & 0.093 & 0.916 & 0.22 & 0.24 & 0.17 & 0.70 & 0.84 & 0.279 & 0.412 & 0.563 & 0.371 & 0.679 & 0.345 & 0.330 & 0.152 & 0.134 & 0.143 & 0.374 & 0.147 & 1.757 & 1.940 & 2.067 & 1.376 & 1.156 & 0.026 & - & DR Congo \\
\hline Ethiopia & - & 0.269 & 0.982 & 0.24 & 0.25 & 0.63 & 0.91 & 0.98 & 0.289 & 0.409 & 0.724 & 0.537 & 0.869 & 0.364 & 0.323 & 0.088 & 0.065 & 0.028 & 0.602 & 0.064 & 1.336 & 1.595 & 1.311 & 1.107 & 1.075 & 0.134 & +1 & Ethiopia \\
\hline Ghana & -3 & 0.062 & 0.711 & 0.06 & 0.06 & 0.23 & 0.70 & 0.60 & 0.241 & 0.306 & 0.666 & 0.335 & 0.543 & 316 & 0.477 & 0.110 & 0.155 & 0.213 & 0.325 & 0.144 & 1.870 & 2.204 & 2.133 & 1.232 & 1.168 & 0.014 & -2 & Shana \\
\hline Haiti & -3 & 0.123 & 0.883 & 0.19 & 0.11 & 0.40 & 0.67 & 0.85 & 0.317 & 0.428 & 0.635 & 0.385 & 0.683 & 0.351 & 0.333 & 0.112 & 0.128 & 0.124 & 0.419 & 0.147 & 1.961 & 2.133 & 1.673 & 1.500 & 1.264 & 0.044 & -1 & Haiti \\
\hline India & +5 & 0.108 & 0.846 & 0.47 & 0.26 & 0.22 & 0.74 & 0.68 & 0.356 & 0.423 & 0.679 & 0.407 & 0.582 & 0.249 & 0.295 & 0.115 & 0.116 & 0.177 & 0.440 & 0.134 & 1.318 & 2.006 & 2.198 & 1.387 & 1.341 & 0.045 & +2 & India \\
\hline Kenya & - & 0.083 & 0.887 & 17 & 0.14 & 0.14 & 0.71 & 0.91 & 0.276 & 0.344 & 0.635 & 0.399 & 0.529 & 0.337 & 0.410 & 0.139 & 0.129 & 0.236 & 0.347 & 0.180 & 2.179 & 2.569 & 2.871 & 1.595 & 1.330 & 0.026 & - & enya \\
\hline Liberia & -7 & 0.130 & 0.904 & 0.14 & 0.18 & 0.33 & 0.69 & 0.79 & 0.238 & 0.377 & 0.734 & 0.370 & 0.807 & 0.365 & 0.358 & 0.091 & 0.129 & 0.043 & 0.398 & 0.141 & 1.646 & 1.611 & 1.900 & 1.288 & 1.198 & 0.042 & -2 & Liberia \\
\hline Malawi & +1 & 0.096 & 0.951 & 0.09 & 0.16 & 0.30 & 0.69 & 0.97 & 0.257 & 0.344 & 0.528 & 0.384 & 0.498 & 0.410 & 0.435 & 0.140 & 0.128 & 0.310 & 0.389 & 0.167 & 1.974 & 2.014 & 2.034 & 1.448 & 1.167 & 0.032 & +2 & Malawi \\
\hline Mali & - & 0.205 & 0.909 & 0.19 & 0.32 & 0.68 & 0.67 & 0.62 & 0.279 & 0.434 & 0.797 & 0.357 & 0.461 & 0.345 & 0.302 & 0.070 & 0.142 & 0.318 & 0.531 & 0.090 & 1.452 & 1.530 & 1.285 & 1.329 & 1.205 & 0.089 & - & Mali \\
\hline Moldova & -1 & 0.007 & 0.228 & 0.21 & 0.03 & 0.16 & 0.20 & 0.59 & 0.234 & 0.175 & 0.391 & 0.240 & 0.466 & 0.363 & 0.534 & 0.286 & 0.264 & 0.296 & 0.181 & 0.249 & 0.931 & 0.764 & 2.863 & 2.121 & 1.121 & 0.001 & -1 & Moldova \\
\hline Morocco & +2 & 0.058 & 0.578 & 0.20 & 0.10 & 0.45 & 0.55 & 0.50 & 0.250 & 0.369 & 0.578 & 0.278 & 0.528 & 0.312 & 0.386 & 0.162 & 0.205 & 0.183 & 0.381 & 0.139 & 1.154 & 2.068 & 1.598 & 1.550 & 1.429 & 0.017 & - & Morocco \\
\hline Mozambique & & 0.135 & 0.938 & 0.10 & 0.19 & 0.60 & 0.61 & 0.96 & 0.205 & 0.382 & 0.573 & 0.334 & 0.555 & 0.431 & 0.403 & 0.144 & 0.156 & 0.245 & 0.472 & 0.133 & 1.558 & 1.861 & 1.417 & 1.525 & 1.093 & 0.054 & - & Mozambique \\
\hline Namibia & +3 & 0.048 & 0.632 & 0.33 & 0.18 & 0.14 & 0.53 & 0.60 & 0.314 & 0.309 & 0.584 & 0.334 & 0.670 & 0.327 & 0.473 & 0.151 & 0.189 & 0.095 & 0.310 & 0.192 & 1.460 & 2.486 & 2.664 & 1.777 & 1.302 & 0.012 & - & Namibia \\
\hline Nepal & +4 & 0.113 & 0.903 & .34 & 0.29 & 0.34 & 0.64 & 0.86 & 0.309 & 0.343 & 0.629 & 0.375 & 0.558 & 0.290 & 0.319 & 0.125 & 0.138 & 0.185 & 0.443 & 0.173 & 1.587 & 2.033 & 1.904 & 1.552 & 1.222 & 0.051 & - & Nepal \\
\hline Niger & - & 0.267 & 0.971 & 0.21 & 0.40 & 0.68 & 0.77 & 0.90 & 0.271 & 0.460 & 0.844 & 0.387 & 0.739 & 0.297 & 0.261 & 0.049 & 0.128 & 0.069 & 0.595 & 0.076 & 1.489 & 1.413 & 1.258 & 1.213 & 1.064 & 0.136 & -1 & Niger \\
\hline Nigeria & -5 & 0.114 & 0.836 & 0.21 & 0.29 & 0.30 & 0.60 & 0.75 & 0.270 & 0.468 & 0.823 & 0.333 & 0.461 & 0.355 & 0.299 & 0.055 & 0.150 & 0.333 & 0.394 & 0.188 & 1.759 & 1.858 & 2.163 & 1.541 & 1.248 & 0.046 & -6 & Nigeria \\
\hline Peru & - & 0.038 & 0.584 & 0.03 & 0.04 & 0.13 & 0.64 & 0.83 & 0.176 & 0.205 & 0.567 & 0.373 & 0.456 & 0.310 & 0.588 & 0.143 & 0.132 & 0.287 & 0.277 & 0.172 & 0.753 & 2.650 & 2.284 & 1.447 & 1.346 & 0.006 & - & Peru \\
\hline Swaziland & - & 0.041 & 0.641 & 0.08 & 0.08 & 0.12 & 0.70 & 0.55 & 0.199 & 0.331 & 0.579 & 0.330 & 0.643 & 0.305 & 0.573 & 0.165 & 0.157 & 0.104 & 0.292 & 0.134 & 1.920 & 1.593 & 2.368 & 1.356 & 1.141 & 0.007 & - & Swaziland \\
\hline Zambia & +1 & 0.079 & 0.839 & 0.12 & 0.16 & 0.16 & 0.83 & 0.79 & 0.264 & 0.285 & 0.490 & 0.422 & 0.524 & 0.396 & 0.444 & 0.188 & 0.120 & 0.276 & 0.376 & 0.117 & 1.711 & 1.927 & 2.532 & 1.243 & 1.238 & 0.021 & +1 & Zambia \\
\hline Zimbabwe & -1 & 0.054 & 0.799 & 0.13 & 0.11 & 0.04 & 0.59 & 0.92 & 0.261 & 0.307 & 0.523 & 0.338 & 0.703 & 0.411 & 0.428 & 0.167 & 0.144 & 0.132 & 0.269 & 0.209 & 2.409 & 2.364 & 2.774 & 1.634 & 1.128 & 0.008 & +1 & Zimbabwe \\
\hline
\end{tabular}




\title{
Appendix $3 \mathrm{~A}$
}

\author{
FIGURES
}

Figure 3.03 Frequency Distribution Satisfaction with Health

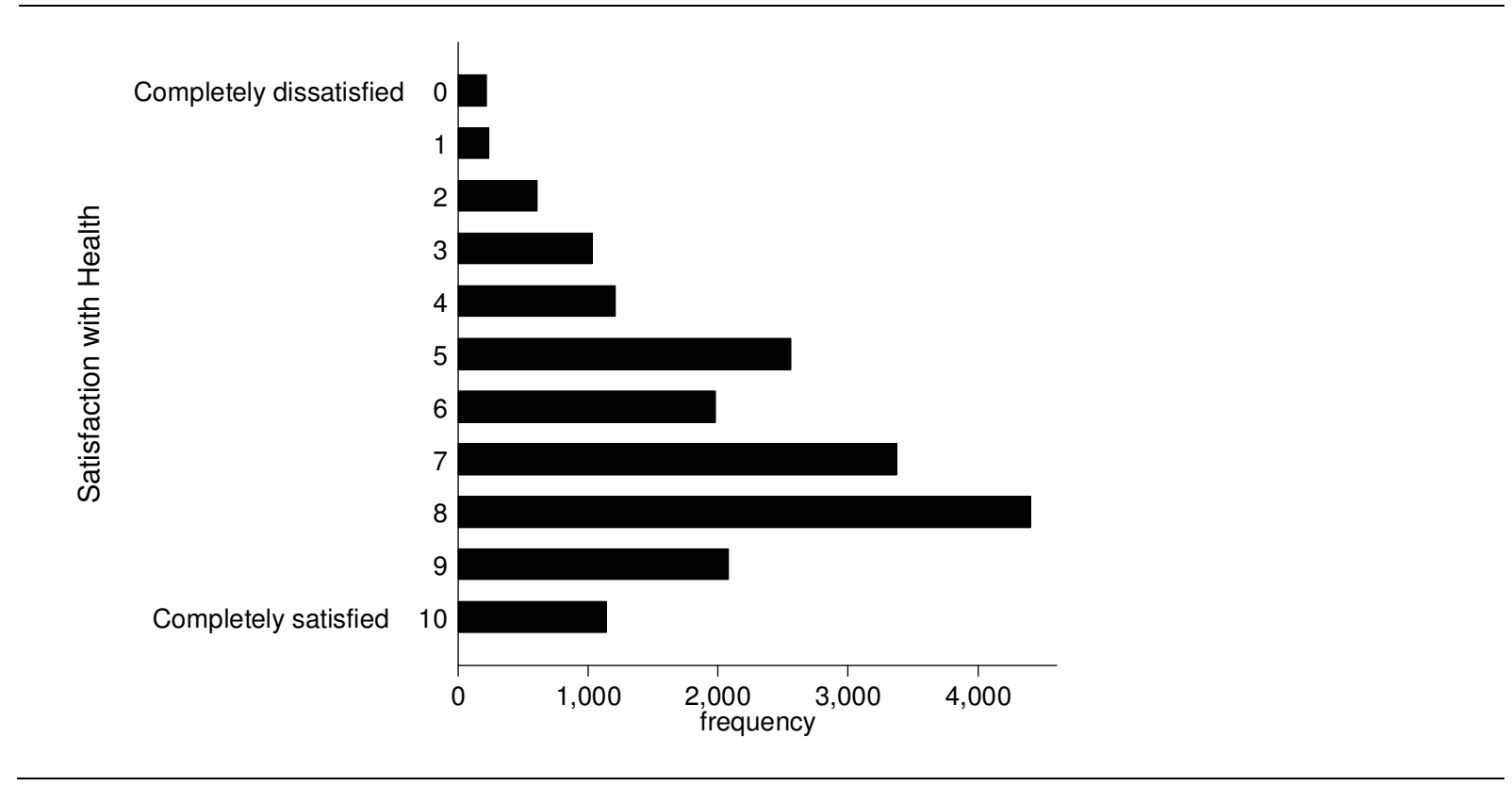

Figure 3.04 Frequency Distribution Satisfaction with Work

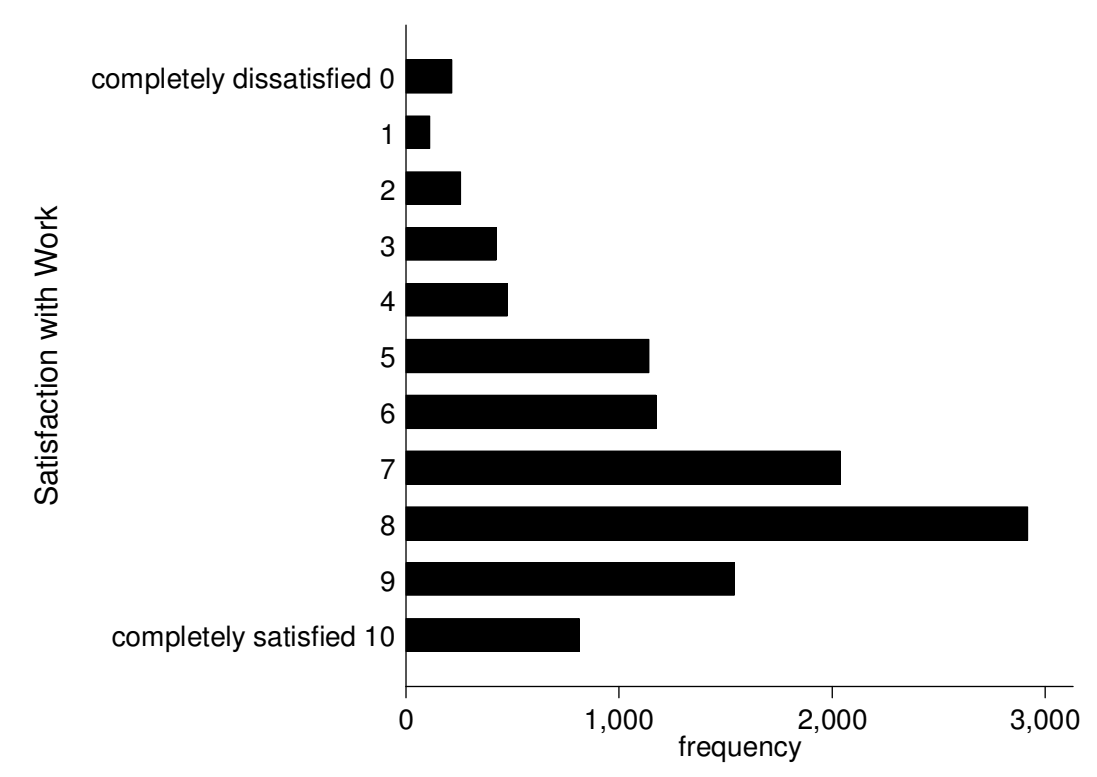


Figure 3.05 Frequency Distribution Satisfaction with Housing

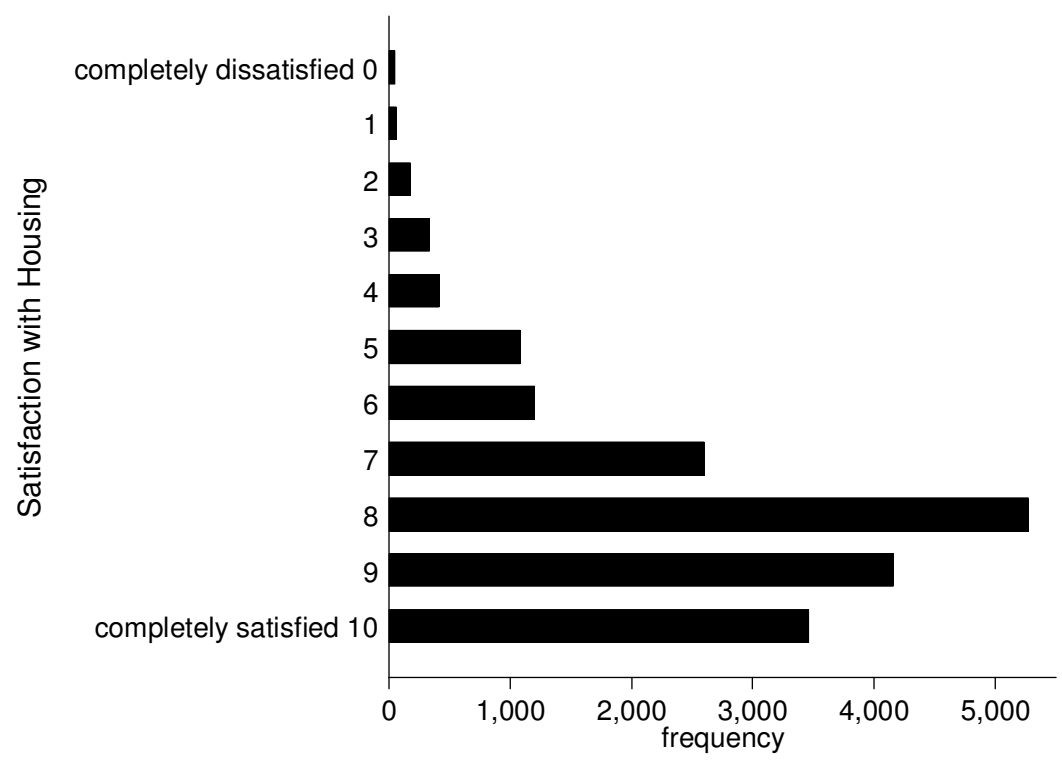

Figure 3.06 Frequency Distribution Satisfaction with Income

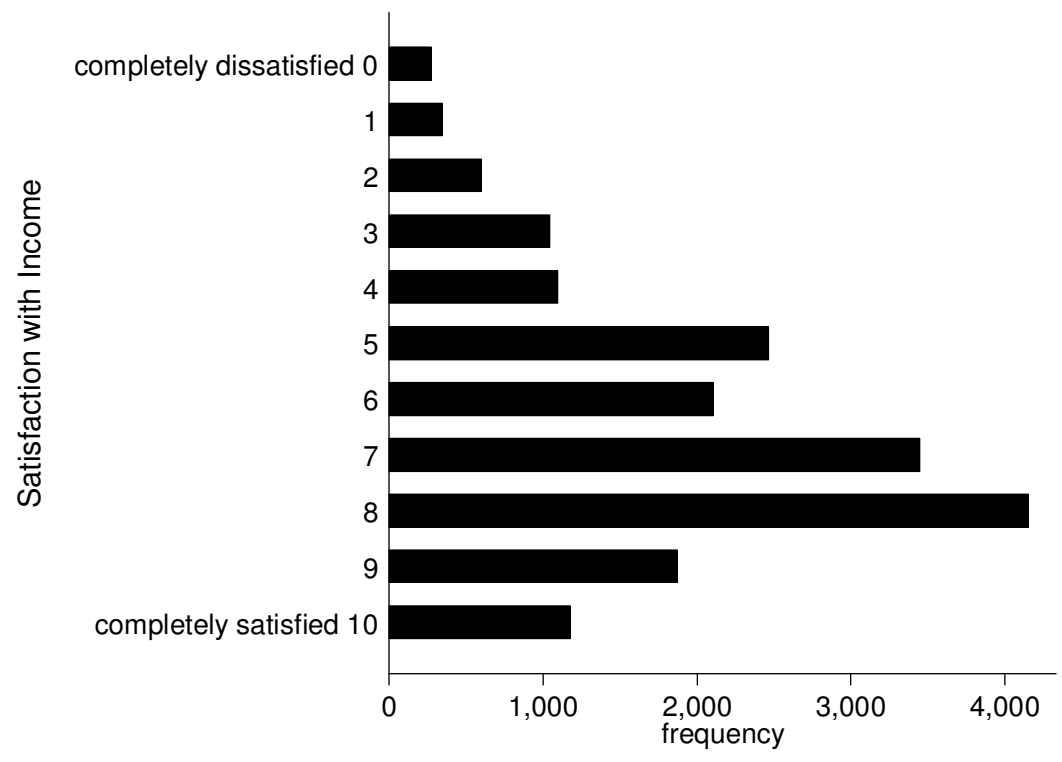




\section{Appendix 3 B}

TABLES 
Table 3.03 GCSPI Decompositions 2002-2010

\begin{tabular}{l}
\hline Bundeslaender \\
\\
\\
\hline Baden-Württemberg \\
Bavaria \\
Berlin \\
Brandenburg \\
Bremen \\
Hamburg \\
Hesse \\
Mecklenburg-W.Pomerania \\
Lower Saxony \\
North Rhine-Westphalia \\
Rhineland-Palatinate \\
Saarland \\
Saxony \\
Saxony-Anhalt \\
Schleswig-Holstein \\
Thuringia \\
\hline All
\end{tabular}
2008 2010

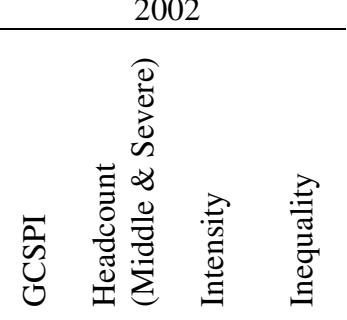

$\begin{array}{llll}0.031 & 0.064 & 0.187 & 0.175\end{array}$ $\begin{array}{llll}0.027 & 0.047 & 0.173 & 0.176\end{array}$ $\begin{array}{llll}0.029 & 0.054 & 0.167 & 0.176\end{array}$ $\begin{array}{llll}0.030 & 0.055 & 0.174 & 0.165 \\ 0.035 & 0.117 & 0.187 & 0.154 \\ 0.026 & 0.038 & 0.161 & 0.205\end{array}$ $\begin{array}{llll}0.035 & 0.117 & 0.187 & 0.154 \\ 0.026 & 0.038 & 0.161 & 0.205\end{array}$ $\begin{array}{llll}0.034 & 0.071 & 0.192 & 0.172\end{array}$ $\begin{array}{lllll}0.033 & 0.072 & 0.184 & 0.190\end{array}$ $\begin{array}{llll}0.024 & 0.047 & 0.168 & 0.163\end{array}$ $\begin{array}{llll}0.028 & 0.057 & 0.175 & 0.170\end{array}$ $\begin{array}{lllll}0.029 & 0.065 & 0.178 & 0.194\end{array}$ $\begin{array}{llll}0.032 & 0.078 & 0.182 & 0.161\end{array}$ $\begin{array}{lllll}0.034 & 0.064 & 0.179 & 0.167\end{array}$ $\begin{array}{lllll}0.032 & 0.079 & 0.180 & 0.175\end{array}$ $\begin{array}{llll}0.022 & 0.043 & 0.160 & 0.188\end{array}$ \begin{tabular}{lllll}
0.031 & 0.055 & 0.177 & 0.171 \\
\hline
\end{tabular} 20042006

(2006

ग)
$\begin{array}{llll}0.032 & 0.078 & 0.188 & 0.172\end{array}$ $\begin{array}{lllll}0.037 & 0.080 & 0.185 & 0.184\end{array}$ $\begin{array}{lllll}0.038 & 0.067 & 0.187 & 0.192\end{array}$ $\begin{array}{lllll}0.031 & 0.089 & 0.177 & 0.192\end{array}$ $\begin{array}{lllll}0.029 & 0.042 & 0.173 & 0.209\end{array}$ $\begin{array}{lllll}0.033 & 0.085 & 0.190 & 0.192\end{array}$ $\begin{array}{llll}0.032 & 0.065 & 0.172 & 0.214\end{array}$ $\begin{array}{llll}0.025 & 0.042 & 0.170 & 0.169\end{array}$ $\begin{array}{lllll}0.032 & 0.072 & 0.182 & 0.190\end{array}$ $\begin{array}{llll}0.033 & 0.074 & 0.190 & 0.171\end{array}$ $\begin{array}{llll}0.035 & 0.086 & 0.195 & 0.179\end{array}$ $\begin{array}{lllll}0.033 & 0.066 & 0.180 & 0.164\end{array}$ $\begin{array}{lllll}0.036 & 0.072 & 0.186 & 0.182\end{array}$ \begin{tabular}{llll}
\hline 0.034 & 0.076 & 0.194 & 0.163
\end{tabular} $\begin{array}{llll}0.033 & 0.079 & 0.190 & 0.185\end{array}$ $\begin{array}{lllll}0.038 & 0.069 & 0.186 & 0.198\end{array}$ $\begin{array}{lllll}0.034 & 0.079 & 0.182 & 0.192\end{array}$ $\begin{array}{lllll}0.024 & 0.033 & 0.172 & 0.118\end{array}$ $\begin{array}{lllll}0.025 & 0.035 & 0.170 & 0.181\end{array}$ $\begin{array}{lllll}0.033 & 0.069 & 0.191 & 0.172\end{array}$ $\begin{array}{lllll}0.029 & 0.046 & 0.168 & 0.160\end{array}$ $\begin{array}{llll}0.028 & 0.053 & 0.175 & 0.188\end{array}$ $\begin{array}{lllll}0.030 & 0.065 & 0.176 & 0.179\end{array}$ $\begin{array}{lllll}0.038 & 0.089 & 0.206 & 0.157\end{array}$ $\begin{array}{lllll}0.044 & 0.129 & 0.206 & 0.208\end{array}$ $\begin{array}{llllll}0.037 & 0.084 & 0.187 & 0.189\end{array}$ $\begin{array}{lllll}0.033 & 0.083 & 0.184 & 0.166\end{array}$ $\begin{array}{lllll}0.022 & 0.032 & 0.166 & 0.142\end{array}$ $\begin{array}{llll}0.024 & 0.035 & 0.167 & 0.177\end{array}$ $\begin{array}{lll}0.042 \quad 0.093 & 0.2\end{array}$

\begin{tabular}{ll}
0.201 & 0.167 \\
\hline
\end{tabular} 
Table 3.04 AROPR Decomposition 2002-2010

\begin{tabular}{|c|c|c|c|c|c|}
\hline Bundeslaender & 2002 & 2004 & 2006 & 2008 & 2010 \\
\hline Baden-Württemberg & 0.094 & 0.078 & $\overline{0.081}$ & 0.080 & 0.088 \\
\hline Bavaria & 0.117 & 0.111 & 0.120 & 0.099 & 0.137 \\
\hline Berlin & 0.146 & 0.185 & 0.171 & 0.188 & 0.201 \\
\hline Brandenburg & 0.142 & 0.160 & 0.190 & 0.220 & 0.218 \\
\hline Bremen & 0.121 & 0.080 & 0.057 & 0.083 & 0.093 \\
\hline Hamburg & 0.077 & 0.103 & 0.041 & 0.058 & 0.038 \\
\hline Hesse & 0.120 & 0.117 & 0.121 & 0.129 & 0.117 \\
\hline Mecklenburg-W.Pomerania & 0.141 & 0.134 & 0.232 & 0.199 & 0.311 \\
\hline Lower Saxony & 0.107 & 0.109 & 0.109 & 0.107 & 0.127 \\
\hline North Rhine-Westphalia & 0.119 & 0.096 & 0.119 & 0.126 & 0.123 \\
\hline Rhineland-Palatinate & 0.131 & 0.123 & 0.150 & 0.134 & 0.163 \\
\hline Saarland & 0.087 & 0.124 & 0.204 & 0.134 & 0.196 \\
\hline Saxony & 0.160 & 0.165 & 0.205 & 0.176 & 0.179 \\
\hline Saxony-Anhalt & 0.149 & 0.133 & 0.188 & 0.211 & 0.186 \\
\hline Schleswig-Holstein & 0.087 & 0.112 & 0.129 & 0.135 & 0.133 \\
\hline Thuringia & 0.181 & 0.171 & 0.224 & 0.217 & 0.194 \\
\hline All & 0.120 & 0.115 & 0.131 & 0.128 & 0.138 \\
\hline
\end{tabular}

Table 3.05 GCSPI Decomposition 2002 (prevalence weights)

\begin{tabular}{|c|c|c|c|c|c|c|c|c|c|c|c|}
\hline \multirow[t]{2}{*}{ Bundeslaender } & \multirow[b]{2}{*}{$\overrightarrow{\tilde{v}}$} & \multirow{2}{*}{ 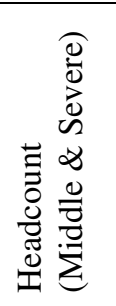 } & \multirow[b]{2}{*}{ 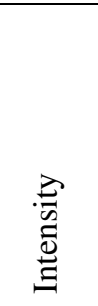 } & \multirow[b]{2}{*}{ 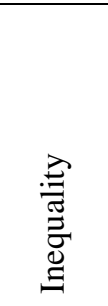 } & \multicolumn{7}{|c|}{ Contribution of Dimensions } \\
\hline & & & & & 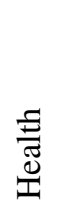 & 总 & 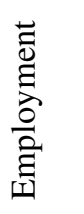 & $\begin{array}{l}\stackrel{00}{\Xi} \\
\stackrel{\Xi}{0} \\
\stackrel{0}{0} \\
\stackrel{0}{I}\end{array}$ & 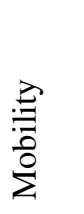 & 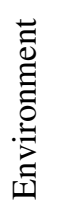 & $\begin{array}{l}\mathscr{\Xi} \\
\stackrel{0}{\Xi} \\
\end{array}$ \\
\hline Baden-Württemberg & 0.031 & $6.4 \%$ & 0.187 & 0.175 & $20 \%$ & $28 \%$ & $21 \%$ & $7 \%$ & $5 \%$ & $9 \%$ & $11 \%$ \\
\hline Bavaria & 0.027 & $4.7 \%$ & 0.173 & 0.176 & $22 \%$ & $25 \%$ & $22 \%$ & $10 \%$ & $3 \%$ & $7 \%$ & $11 \%$ \\
\hline Berlin & 0.029 & $5.4 \%$ & 0.167 & 0.176 & $20 \%$ & $11 \%$ & $30 \%$ & $11 \%$ & $10 \%$ & $9 \%$ & $10 \%$ \\
\hline Brandenburg & 0.030 & $5.5 \%$ & 0.174 & 0.165 & $23 \%$ & $12 \%$ & $30 \%$ & $10 \%$ & $7 \%$ & $7 \%$ & $10 \%$ \\
\hline Bremen & 0.035 & $11.7 \%$ & 0.187 & 0.154 & $23 \%$ & $18 \%$ & $22 \%$ & $5 \%$ & $11 \%$ & $8 \%$ & $14 \%$ \\
\hline Hamburg & 0.026 & $3.8 \%$ & 0.161 & 0.205 & $18 \%$ & $20 \%$ & $22 \%$ & $8 \%$ & $9 \%$ & $16 \%$ & $7 \%$ \\
\hline Hesse & 0.034 & $7.1 \%$ & 0.192 & 0.172 & $21 \%$ & $22 \%$ & $23 \%$ & $8 \%$ & $5 \%$ & $7 \%$ & $14 \%$ \\
\hline Mecklenburg-W.Pomerania & 0.033 & $7.2 \%$ & 0.184 & 0.190 & $22 \%$ & $13 \%$ & $29 \%$ & $9 \%$ & $7 \%$ & $5 \%$ & $15 \%$ \\
\hline Lower Saxony & 0.024 & $4.7 \%$ & 0.168 & 0.163 & $22 \%$ & $25 \%$ & $25 \%$ & $6 \%$ & $5 \%$ & $6 \%$ & $12 \%$ \\
\hline North Rhine-Westphalia & 0.028 & $5.7 \%$ & 0.175 & 0.170 & $22 \%$ & $25 \%$ & $22 \%$ & $7 \%$ & $7 \%$ & $6 \%$ & $12 \%$ \\
\hline Rhineland-Palatinate & 0.029 & $6.5 \%$ & 0.178 & 0.194 & $25 \%$ & $27 \%$ & $20 \%$ & $6 \%$ & $5 \%$ & $7 \%$ & $10 \%$ \\
\hline Saarland & 0.032 & $7.8 \%$ & 0.182 & 0.161 & $24 \%$ & $26 \%$ & $23 \%$ & $7 \%$ & $1 \%$ & $6 \%$ & $13 \%$ \\
\hline Saxony & 0.034 & $6.4 \%$ & 0.179 & 0.167 & $20 \%$ & $12 \%$ & $31 \%$ & $12 \%$ & $4 \%$ & $6 \%$ & $15 \%$ \\
\hline Saxony-Anhalt & 0.032 & $7.9 \%$ & 0.180 & 0.175 & $21 \%$ & $12 \%$ & $33 \%$ & $11 \%$ & $7 \%$ & $6 \%$ & $11 \%$ \\
\hline Schleswig-Holstein & 0.022 & $4.3 \%$ & 0.160 & 0.188 & $20 \%$ & $27 \%$ & $26 \%$ & $6 \%$ & $5 \%$ & $7 \%$ & $9 \%$ \\
\hline Thuringia & 0.031 & $5.5 \%$ & 0.177 & 0.171 & $24 \%$ & $11 \%$ & $31 \%$ & $10 \%$ & $4 \%$ & $6 \%$ & $14 \%$ \\
\hline All & 0.029 & $5.8 \%$ & 0.177 & 0.175 & $22 \%$ & $22 \%$ & $25 \%$ & $8 \%$ & $6 \%$ & $7 \%$ & $12 \%$ \\
\hline
\end{tabular}


Table 3.06 GCSPI Decomposition 2004 (prevalence weights)

\begin{tabular}{|c|c|c|c|c|c|c|c|c|c|c|c|}
\hline \multirow[t]{2}{*}{ Bundeslaender } & \multirow[b]{2}{*}{$\underset{v}{\tilde{v}}$} & \multirow{2}{*}{ 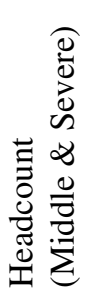 } & \multirow[b]{2}{*}{ 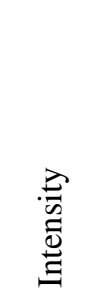 } & \multirow[b]{2}{*}{ 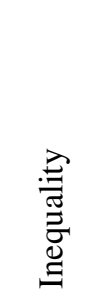 } & \multicolumn{7}{|c|}{ Contribution of Dimensions } \\
\hline & & & & & 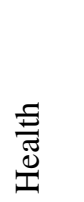 & 芯 & 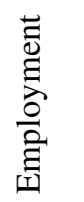 & 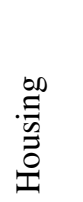 & 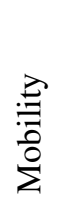 & $\begin{array}{l}\underset{\Xi}{\Xi} \\
\Xi \\
\stackrel{\Xi}{\Xi} \\
\underset{\Xi}{\Xi}\end{array}$ & $\begin{array}{l}\mathscr{\Xi} \\
\text { ठ } \\
\Xi\end{array}$ \\
\hline Baden-Württemberg & 0.033 & $7.6 \%$ & 0.191 & 0.187 & $20 \%$ & $26 \%$ & $21 \%$ & $8 \%$ & $5 \%$ & $9 \%$ & $12 \%$ \\
\hline Bavaria & 0.032 & $7.8 \%$ & 0.188 & 0.172 & $20 \%$ & $23 \%$ & $25 \%$ & $8 \%$ & $3 \%$ & $8 \%$ & $13 \%$ \\
\hline Berlin & 0.037 & $8.0 \%$ & 0.185 & 0.184 & $20 \%$ & $10 \%$ & $30 \%$ & $10 \%$ & $8 \%$ & $8 \%$ & $15 \%$ \\
\hline Brandenburg & 0.038 & $6.7 \%$ & 0.187 & 0.192 & $21 \%$ & $12 \%$ & $28 \%$ & $10 \%$ & $7 \%$ & $6 \%$ & $16 \%$ \\
\hline Bremen & 0.031 & $8.9 \%$ & 0.177 & 0.192 & $23 \%$ & $20 \%$ & $24 \%$ & $7 \%$ & $11 \%$ & $5 \%$ & $11 \%$ \\
\hline Hamburg & 0.029 & $4.2 \%$ & 0.173 & 0.209 & $21 \%$ & $16 \%$ & $23 \%$ & $8 \%$ & $8 \%$ & $14 \%$ & $11 \%$ \\
\hline Hesse & 0.033 & $8.5 \%$ & 0.190 & 0.192 & $21 \%$ & $21 \%$ & $24 \%$ & $9 \%$ & $7 \%$ & $8 \%$ & $11 \%$ \\
\hline Mecklenburg-W.Pomerania & 0.032 & $6.5 \%$ & 0.172 & 0.214 & $20 \%$ & $12 \%$ & $32 \%$ & $8 \%$ & $7 \%$ & $9 \%$ & $13 \%$ \\
\hline Lower Saxony & 0.025 & $4.2 \%$ & 0.170 & 0.169 & $25 \%$ & $25 \%$ & $23 \%$ & $5 \%$ & $5 \%$ & $4 \%$ & $12 \%$ \\
\hline North Rhine-Westphalia & 0.032 & $7.2 \%$ & 0.182 & 0.190 & $22 \%$ & $22 \%$ & $24 \%$ & $7 \%$ & $6 \%$ & $5 \%$ & $13 \%$ \\
\hline Rhineland-Palatinate & 0.033 & $7.4 \%$ & 0.190 & 0.171 & $19 \%$ & $26 \%$ & $21 \%$ & $6 \%$ & $5 \%$ & $10 \%$ & $13 \%$ \\
\hline Saarland & 0.035 & $8.6 \%$ & 0.195 & 0.179 & $21 \%$ & $24 \%$ & $24 \%$ & $9 \%$ & $3 \%$ & $5 \%$ & $13 \%$ \\
\hline Saxony & 0.033 & $6.6 \%$ & 0.180 & 0.164 & $20 \%$ & $12 \%$ & $32 \%$ & $11 \%$ & $4 \%$ & $6 \%$ & $15 \%$ \\
\hline Saxony-Anhalt & 0.036 & $7.2 \%$ & 0.186 & 0.182 & $19 \%$ & $14 \%$ & $30 \%$ & $10 \%$ & $6 \%$ & $7 \%$ & $13 \%$ \\
\hline Schleswig-Holstein & 0.024 & $3.5 \%$ & 0.167 & 0.177 & $18 \%$ & $22 \%$ & $30 \%$ & $8 \%$ & $5 \%$ & $5 \%$ & $11 \%$ \\
\hline Thuringia & 0.035 & $7.0 \%$ & 0.185 & 0.168 & $20 \%$ & $10 \%$ & $31 \%$ & $12 \%$ & $6 \%$ & $5 \%$ & $16 \%$ \\
\hline All & 0.032 & $7.0 \%$ & 0.184 & 0.183 & $21 \%$ & $21 \%$ & $25 \%$ & $8 \%$ & $5 \%$ & $7 \%$ & $13 \%$ \\
\hline
\end{tabular}

Table 3.07 GCSPI Decomposition 2006 (prevalence weights)

\begin{tabular}{|c|c|c|c|c|c|c|c|c|c|c|c|}
\hline \multirow[t]{2}{*}{ Bundeslaender } & \multirow[b]{2}{*}{$\underset{v}{\tilde{v}}$} & \multirow{2}{*}{ 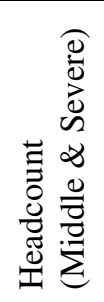 } & \multirow[b]{2}{*}{ 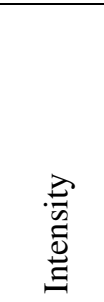 } & \multirow[b]{2}{*}{$\begin{array}{l}\stackrel{\Xi}{\Xi} \\
\stackrel{\Xi}{\Xi} \\
\stackrel{\Xi}{\Xi}\end{array}$} & \multicolumn{7}{|c|}{ Contribution of Dimensions } \\
\hline & & & & & 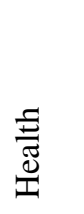 & 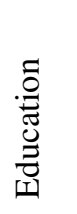 & 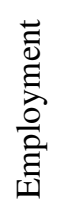 & $\begin{array}{l}\stackrel{\infty}{\Xi} \\
\stackrel{\Xi}{0} \\
\stackrel{0}{0} \\
\stackrel{0}{I}\end{array}$ & 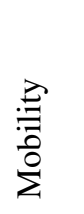 & 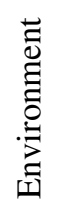 & $\begin{array}{l}\stackrel{\Xi}{\Xi} \\
\stackrel{0}{\Xi} \\
\Xi\end{array}$ \\
\hline Baden-Württemberg & 0.034 & $7.6 \%$ & 0.194 & 0.163 & $20 \%$ & $26 \%$ & $21 \%$ & $8 \%$ & $5 \%$ & $10 \%$ & $9 \%$ \\
\hline Bavaria & 0.033 & $7.9 \%$ & 0.190 & 0.185 & $21 \%$ & $24 \%$ & $24 \%$ & $9 \%$ & $3 \%$ & $7 \%$ & $12 \%$ \\
\hline Berlin & 0.038 & $6.9 \%$ & 0.186 & 0.198 & $22 \%$ & $13 \%$ & $32 \%$ & $8 \%$ & $9 \%$ & $5 \%$ & $12 \%$ \\
\hline Brandenburg & 0.034 & $7.9 \%$ & 0.182 & 0.192 & $21 \%$ & $9 \%$ & $30 \%$ & $9 \%$ & $6 \%$ & $7 \%$ & $19 \%$ \\
\hline Bremen & 0.024 & $3.3 \%$ & 0.172 & 0.118 & $27 \%$ & $27 \%$ & $24 \%$ & $3 \%$ & $11 \%$ & $5 \%$ & $3 \%$ \\
\hline Hamburg & 0.025 & $3.5 \%$ & 0.170 & 0.181 & $22 \%$ & $16 \%$ & $22 \%$ & $9 \%$ & $12 \%$ & $7 \%$ & $11 \%$ \\
\hline Hesse & 0.033 & $6.9 \%$ & 0.191 & 0.172 & $24 \%$ & $19 \%$ & $25 \%$ & $7 \%$ & $6 \%$ & $7 \%$ & $11 \%$ \\
\hline Mecklenburg-W.Pomerania & 0.029 & $4.6 \%$ & 0.168 & 0.160 & $21 \%$ & $11 \%$ & $31 \%$ & $8 \%$ & $6 \%$ & $7 \%$ & $16 \%$ \\
\hline Lower Saxony & 0.028 & $5.3 \%$ & 0.175 & 0.188 & $22 \%$ & $24 \%$ & $25 \%$ & $5 \%$ & $5 \%$ & $4 \%$ & $15 \%$ \\
\hline North Rhine-Westphalia & 0.030 & $6.5 \%$ & 0.176 & 0.179 & $22 \%$ & $25 \%$ & $24 \%$ & $7 \%$ & $7 \%$ & $5 \%$ & $10 \%$ \\
\hline Rhineland-Palatinate & 0.038 & $8.9 \%$ & 0.206 & 0.157 & $20 \%$ & $21 \%$ & $22 \%$ & $7 \%$ & $4 \%$ & $12 \%$ & $15 \%$ \\
\hline Saarland & 0.044 & $12.9 \%$ & 0.206 & 0.208 & $16 \%$ & $17 \%$ & $29 \%$ & $15 \%$ & $2 \%$ & $4 \%$ & $17 \%$ \\
\hline Saxony & 0.037 & $8.4 \%$ & 0.187 & 0.189 & $22 \%$ & $9 \%$ & $31 \%$ & $9 \%$ & $4 \%$ & $8 \%$ & $17 \%$ \\
\hline Saxony-Anhalt & 0.033 & $8.3 \%$ & 0.184 & 0.166 & $23 \%$ & $11 \%$ & $32 \%$ & $10 \%$ & $6 \%$ & $3 \%$ & $16 \%$ \\
\hline Schleswig-Holstein & 0.022 & $3.2 \%$ & 0.166 & 0.142 & $28 \%$ & $20 \%$ & $26 \%$ & $5 \%$ & $4 \%$ & $5 \%$ & $12 \%$ \\
\hline Thuringia & 0.042 & $9.3 \%$ & 0.201 & 0.167 & $21 \%$ & $9 \%$ & $29 \%$ & $10 \%$ & $4 \%$ & $5 \%$ & $21 \%$ \\
\hline All & 0.032 & $7.1 \%$ & 0.185 & 0.179 & $22 \%$ & $20 \%$ & $25 \%$ & $8 \%$ & $5 \%$ & $6 \%$ & $13 \%$ \\
\hline
\end{tabular}


Table 3.08 GCSPI Decomposition 2008 (prevalence weights)

\begin{tabular}{|c|c|c|c|c|c|c|c|c|c|c|c|}
\hline \multirow[t]{2}{*}{ Bundeslaender } & \multirow[b]{2}{*}{$\underset{v}{\tilde{v}}$} & \multirow{2}{*}{ 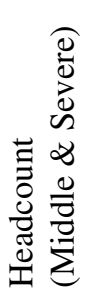 } & \multirow[b]{2}{*}{ 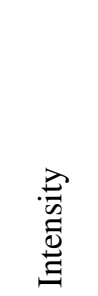 } & \multirow[b]{2}{*}{ 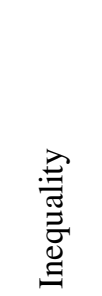 } & \multicolumn{7}{|c|}{ Contribution of Dimensions } \\
\hline & & & & & 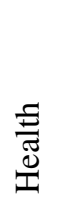 & 芯 & 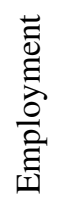 & 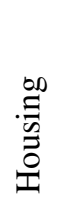 & 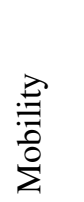 & $\begin{array}{l}\underset{\Xi}{\Xi} \\
\Xi \\
\stackrel{\Xi}{\Xi} \\
\underset{\Xi}{\Xi}\end{array}$ & $\begin{array}{l}\mathscr{\Xi} \\
\text { ठ } \\
\Xi\end{array}$ \\
\hline Baden-Württemberg & 0.029 & $6.3 \%$ & 0.182 & 0.165 & $18 \%$ & $24 \%$ & $28 \%$ & $7 \%$ & $4 \%$ & $8 \%$ & $11 \%$ \\
\hline Bavaria & 0.027 & $5.9 \%$ & 0.178 & 0.160 & $20 \%$ & $22 \%$ & $28 \%$ & $9 \%$ & $3 \%$ & $7 \%$ & $11 \%$ \\
\hline Berlin & 0.040 & $7.1 \%$ & 0.197 & 0.180 & $18 \%$ & $12 \%$ & $33 \%$ & $8 \%$ & $7 \%$ & $7 \%$ & $15 \%$ \\
\hline Brandenburg & 0.036 & $8.7 \%$ & 0.194 & 0.189 & $17 \%$ & $13 \%$ & $32 \%$ & $7 \%$ & $4 \%$ & $3 \%$ & $23 \%$ \\
\hline Bremen & 0.025 & $0.7 \%$ & 0.176 & 0.102 & $14 \%$ & $22 \%$ & $25 \%$ & $10 \%$ & $17 \%$ & $9 \%$ & $3 \%$ \\
\hline Hamburg & 0.020 & $3.8 \%$ & 0.143 & 0.227 & $15 \%$ & $15 \%$ & $34 \%$ & $9 \%$ & $8 \%$ & $10 \%$ & $10 \%$ \\
\hline Hesse & 0.032 & $8.1 \%$ & 0.187 & 0.210 & $21 \%$ & $20 \%$ & $28 \%$ & $7 \%$ & $6 \%$ & $9 \%$ & $10 \%$ \\
\hline Mecklenburg-W.Pomerania & 0.033 & $6.8 \%$ & 0.185 & 0.163 & $17 \%$ & $10 \%$ & $36 \%$ & $7 \%$ & $3 \%$ & $4 \%$ & $22 \%$ \\
\hline Lower Saxony & 0.025 & $4.7 \%$ & 0.173 & 0.169 & $21 \%$ & $22 \%$ & $30 \%$ & $7 \%$ & $4 \%$ & $6 \%$ & $11 \%$ \\
\hline North Rhine-Westphalia & 0.031 & $7.1 \%$ & 0.183 & 0.179 & $20 \%$ & $20 \%$ & $28 \%$ & $7 \%$ & $6 \%$ & $8 \%$ & $12 \%$ \\
\hline Rhineland-Palatinate & 0.030 & $6.7 \%$ & 0.193 & 0.156 & $19 \%$ & $25 \%$ & $30 \%$ & $6 \%$ & $3 \%$ & $4 \%$ & $13 \%$ \\
\hline Saarland & 0.038 & $9.0 \%$ & 0.208 & 0.217 & $17 \%$ & $20 \%$ & $24 \%$ & $9 \%$ & $8 \%$ & $9 \%$ & $14 \%$ \\
\hline Saxony & 0.037 & $6.3 \%$ & 0.190 & 0.185 & $21 \%$ & $9 \%$ & $33 \%$ & $9 \%$ & $6 \%$ & $7 \%$ & $16 \%$ \\
\hline Saxony-Anhalt & 0.038 & $9.5 \%$ & 0.192 & 0.184 & $18 \%$ & $12 \%$ & $34 \%$ & $8 \%$ & $5 \%$ & $3 \%$ & $20 \%$ \\
\hline Schleswig-Holstein & 0.027 & $4.8 \%$ & 0.178 & 0.174 & $22 \%$ & $21 \%$ & $27 \%$ & $6 \%$ & $3 \%$ & $4 \%$ & $16 \%$ \\
\hline Thuringia & 0.034 & $5.8 \%$ & 0.189 & 0.149 & $20 \%$ & $9 \%$ & $34 \%$ & $9 \%$ & $3 \%$ & $4 \%$ & $21 \%$ \\
\hline All & 0.031 & $6.5 \%$ & 0.183 & 0.176 & $19 \%$ & $19 \%$ & $30 \%$ & $8 \%$ & $5 \%$ & $7 \%$ & $13 \%$ \\
\hline
\end{tabular}

Table 3.09 GCSPI Decomposition 2010 (prevalence weights)

\begin{tabular}{|c|c|c|c|c|c|c|c|c|c|c|c|}
\hline \multirow[t]{2}{*}{ Bundeslaender } & \multirow[b]{2}{*}{$\underset{v}{\tilde{v}}$} & \multirow{2}{*}{ 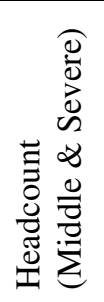 } & \multirow[b]{2}{*}{ 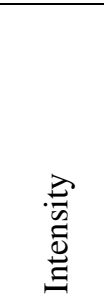 } & \multirow[b]{2}{*}{$\begin{array}{l}\stackrel{\Xi}{\Xi} \\
\stackrel{\Xi}{\Xi} \\
\stackrel{\Xi}{\Xi}\end{array}$} & \multicolumn{7}{|c|}{ Contribution of Dimensions } \\
\hline & & & & & 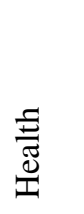 & 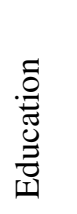 & 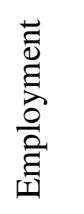 & $\begin{array}{l}\stackrel{\infty}{\Xi} \\
\stackrel{\Xi}{0} \\
\stackrel{0}{0} \\
\stackrel{0}{I}\end{array}$ & 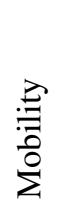 & 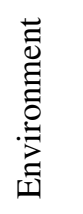 & $\begin{array}{l}\stackrel{\Xi}{\Xi} \\
\stackrel{0}{\Xi} \\
\Xi\end{array}$ \\
\hline Baden-Württemberg & 0.027 & $5.7 \%$ & 0.175 & 0.165 & $22 \%$ & $24 \%$ & $27 \%$ & $7 \%$ & $4 \%$ & $7 \%$ & $9 \%$ \\
\hline Bavaria & 0.032 & $6.9 \%$ & 0.186 & 0.184 & $22 \%$ & $21 \%$ & $25 \%$ & $10 \%$ & $3 \%$ & $4 \%$ & $15 \%$ \\
\hline Berlin & 0.047 & $10.7 \%$ & 0.207 & 0.211 & $24 \%$ & $13 \%$ & $25 \%$ & $9 \%$ & $6 \%$ & $10 \%$ & $14 \%$ \\
\hline Brandenburg & 0.031 & $7.2 \%$ & 0.173 & 0.207 & $18 \%$ & $10 \%$ & $33 \%$ & $8 \%$ & $6 \%$ & $4 \%$ & $21 \%$ \\
\hline Bremen & 0.028 & $4.7 \%$ & 0.184 & 0.102 & $24 \%$ & $17 \%$ & $23 \%$ & $2 \%$ & $16 \%$ & $15 \%$ & $3 \%$ \\
\hline Hamburg & 0.018 & $2.9 \%$ & 0.133 & 0.224 & $17 \%$ & $16 \%$ & $36 \%$ & $5 \%$ & $7 \%$ & $10 \%$ & $9 \%$ \\
\hline Hesse & 0.029 & $6.2 \%$ & 0.184 & 0.181 & $22 \%$ & $17 \%$ & $28 \%$ & $8 \%$ & $5 \%$ & $9 \%$ & $11 \%$ \\
\hline Mecklenburg-W.Pomerania & 0.032 & $7.0 \%$ & 0.185 & 0.127 & $24 \%$ & $7 \%$ & $31 \%$ & $7 \%$ & $4 \%$ & $5 \%$ & $22 \%$ \\
\hline Lower Saxony & 0.027 & $5.4 \%$ & 0.179 & 0.192 & $20 \%$ & $20 \%$ & $28 \%$ & $6 \%$ & $4 \%$ & $6 \%$ & $15 \%$ \\
\hline North Rhine-Westphalia & 0.029 & $5.6 \%$ & 0.176 & 0.183 & $23 \%$ & $21 \%$ & $27 \%$ & $6 \%$ & $6 \%$ & $5 \%$ & $11 \%$ \\
\hline Rhineland-Palatinate & 0.035 & $10.6 \%$ & 0.187 & 0.209 & $17 \%$ & $24 \%$ & $29 \%$ & $5 \%$ & $4 \%$ & $3 \%$ & $19 \%$ \\
\hline Saarland & 0.040 & $16.6 \%$ & 0.213 & 0.181 & $13 \%$ & $18 \%$ & $28 \%$ & $12 \%$ & $5 \%$ & $6 \%$ & $19 \%$ \\
\hline Saxony & 0.037 & $9.9 \%$ & 0.190 & 0.196 & $21 \%$ & $9 \%$ & $32 \%$ & $10 \%$ & $7 \%$ & $6 \%$ & $16 \%$ \\
\hline Saxony-Anhalt & 0.035 & $7.5 \%$ & 0.182 & 0.233 & $21 \%$ & $13 \%$ & $32 \%$ & $9 \%$ & $4 \%$ & $4 \%$ & $17 \%$ \\
\hline Schleswig-Holstein & 0.022 & $2.6 \%$ & 0.165 & 0.148 & $27 \%$ & $20 \%$ & $27 \%$ & $8 \%$ & $4 \%$ & $5 \%$ & $9 \%$ \\
\hline Thuringia & 0.037 & $7.9 \%$ & 0.197 & 0.164 & $20 \%$ & $9 \%$ & $34 \%$ & $8 \%$ & $3 \%$ & $5 \%$ & $21 \%$ \\
\hline All & 0.031 & $6.7 \%$ & 0.183 & 0.176 & $22 \%$ & $18 \%$ & $28 \%$ & $8 \%$ & $5 \%$ & $6 \%$ & $14 \%$ \\
\hline
\end{tabular}


Table 3.10 GCSPI Decomposition 2002 (equal weights)

\begin{tabular}{|c|c|c|c|c|c|c|c|c|c|c|c|}
\hline \multirow[t]{2}{*}{ Bundeslaender } & \multirow[b]{2}{*}{$\overrightarrow{\tilde{v}}$} & \multirow{2}{*}{ 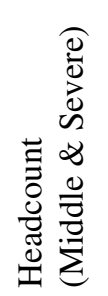 } & \multirow[b]{2}{*}{ 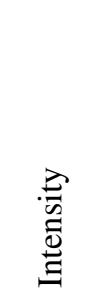 } & \multirow[b]{2}{*}{ 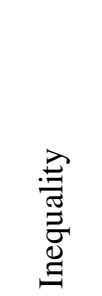 } & \multicolumn{7}{|c|}{ Contribution of Dimensions } \\
\hline & & & & & 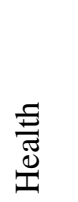 & 芯 & 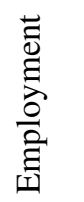 & 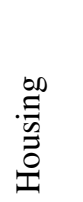 & 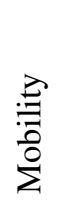 & $\begin{array}{l}\underset{\Xi}{\Xi} \\
\Xi \\
\stackrel{\Xi}{\Xi} \\
\underset{\Xi}{\Xi}\end{array}$ & $\begin{array}{l}\mathscr{\Xi} \\
\text { ठ } \\
\Xi\end{array}$ \\
\hline Baden-Württemberg & 0.032 & $8.1 \%$ & 0.188 & 0.174 & $20 \%$ & $27 \%$ & $21 \%$ & $7 \%$ & $5 \%$ & $9 \%$ & $11 \%$ \\
\hline Bavaria & 0.027 & $6.2 \%$ & 0.175 & 0.175 & $22 \%$ & $25 \%$ & $22 \%$ & $10 \%$ & $4 \%$ & $7 \%$ & $11 \%$ \\
\hline Berlin & 0.030 & $7.5 \%$ & 0.170 & 0.173 & $19 \%$ & $11 \%$ & $29 \%$ & $11 \%$ & $10 \%$ & $9 \%$ & $10 \%$ \\
\hline Brandenburg & 0.030 & $7.2 \%$ & 0.176 & 0.163 & $23 \%$ & $12 \%$ & $30 \%$ & $10 \%$ & $7 \%$ & $7 \%$ & $10 \%$ \\
\hline Bremen & 0.036 & $11.7 \%$ & 0.189 & 0.152 & $23 \%$ & $18 \%$ & $21 \%$ & $5 \%$ & $11 \%$ & $8 \%$ & $14 \%$ \\
\hline Hamburg & 0.026 & $7.9 \%$ & 0.164 & 0.198 & $17 \%$ & $20 \%$ & $22 \%$ & $9 \%$ & $10 \%$ & $16 \%$ & $6 \%$ \\
\hline Hesse & 0.034 & $9.4 \%$ & 0.193 & 0.170 & $21 \%$ & $22 \%$ & $22 \%$ & $8 \%$ & $6 \%$ & $7 \%$ & $14 \%$ \\
\hline Mecklenburg-W.Pomerania & 0.034 & $9.1 \%$ & 0.187 & 0.188 & $22 \%$ & $13 \%$ & $28 \%$ & $10 \%$ & $7 \%$ & $5 \%$ & $15 \%$ \\
\hline Lower Saxony & 0.024 & $5.9 \%$ & 0.169 & 0.161 & $21 \%$ & $25 \%$ & $25 \%$ & $6 \%$ & $5 \%$ & $6 \%$ & $12 \%$ \\
\hline North Rhine-Westphalia & 0.028 & $7.1 \%$ & 0.176 & 0.168 & $22 \%$ & $24 \%$ & $22 \%$ & $7 \%$ & $7 \%$ & $6 \%$ & $12 \%$ \\
\hline Rhineland-Palatinate & 0.029 & $7.3 \%$ & 0.179 & 0.192 & $25 \%$ & $27 \%$ & $20 \%$ & $6 \%$ & $5 \%$ & $8 \%$ & $10 \%$ \\
\hline Saarland & 0.032 & $8.6 \%$ & 0.183 & 0.160 & $24 \%$ & $26 \%$ & $23 \%$ & $7 \%$ & $1 \%$ & $6 \%$ & $13 \%$ \\
\hline Saxony & 0.034 & $9.1 \%$ & 0.181 & 0.163 & $20 \%$ & $12 \%$ & $31 \%$ & $12 \%$ & $5 \%$ & $6 \%$ & $15 \%$ \\
\hline Saxony-Anhalt & 0.033 & $9.4 \%$ & 0.182 & 0.172 & $21 \%$ & $12 \%$ & $33 \%$ & $11 \%$ & $7 \%$ & $6 \%$ & $11 \%$ \\
\hline Schleswig-Holstein & 0.022 & $5.5 \%$ & 0.161 & 0.184 & $19 \%$ & $27 \%$ & $26 \%$ & $7 \%$ & $5 \%$ & $7 \%$ & $9 \%$ \\
\hline Thuringia & 0.032 & $8.5 \%$ & 0.178 & 0.169 & $23 \%$ & $11 \%$ & $31 \%$ & $11 \%$ & $4 \%$ & $6 \%$ & $14 \%$ \\
\hline All & 0.029 & $7.5 \%$ & 0.178 & 0.173 & $21 \%$ & $22 \%$ & $24 \%$ & $8 \%$ & $6 \%$ & $7 \%$ & $12 \%$ \\
\hline
\end{tabular}

Table 3.11 GCSPI Decomposition 2004 (equal weights)

\begin{tabular}{|c|c|c|c|c|c|c|c|c|c|c|c|}
\hline \multirow[t]{2}{*}{ Bundeslaender } & \multirow[b]{2}{*}{$\underset{v}{\tilde{v}}$} & \multirow{2}{*}{ 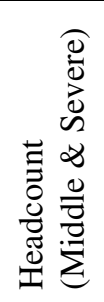 } & \multirow[b]{2}{*}{ 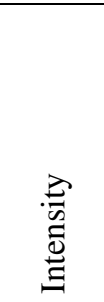 } & \multirow[b]{2}{*}{$\begin{array}{l}\stackrel{\Xi}{\Xi} \\
\stackrel{\Xi}{\Xi} \\
\stackrel{\Xi}{\Xi}\end{array}$} & \multicolumn{7}{|c|}{ Contribution of Dimensions } \\
\hline & & & & & 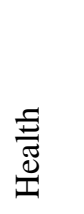 & 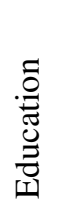 & 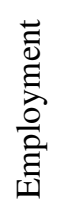 & $\begin{array}{l}\stackrel{\infty}{\Xi} \\
\stackrel{\Xi}{0} \\
\stackrel{0}{0} \\
\stackrel{0}{I}\end{array}$ & 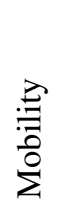 & 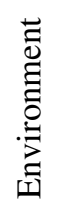 & $\begin{array}{l}\stackrel{\Xi}{\Xi} \\
\stackrel{0}{\Xi} \\
\Xi\end{array}$ \\
\hline Baden-Württemberg & 0.034 & $9.0 \%$ & 0.192 & 0.187 & $20 \%$ & $26 \%$ & $21 \%$ & $8 \%$ & $5 \%$ & $9 \%$ & $12 \%$ \\
\hline Bavaria & 0.032 & $9.3 \%$ & 0.189 & 0.171 & $20 \%$ & $23 \%$ & $25 \%$ & $8 \%$ & $3 \%$ & $8 \%$ & $13 \%$ \\
\hline Berlin & 0.037 & $11.5 \%$ & 0.187 & 0.182 & $19 \%$ & $10 \%$ & $29 \%$ & $10 \%$ & $9 \%$ & $8 \%$ & $14 \%$ \\
\hline Brandenburg & 0.038 & $10.0 \%$ & 0.189 & 0.191 & $21 \%$ & $12 \%$ & $27 \%$ & $11 \%$ & $7 \%$ & $6 \%$ & $16 \%$ \\
\hline Bremen & 0.031 & $12.6 \%$ & 0.178 & 0.191 & $22 \%$ & $20 \%$ & $24 \%$ & $7 \%$ & $11 \%$ & $5 \%$ & $11 \%$ \\
\hline Hamburg & 0.029 & $5.9 \%$ & 0.175 & 0.206 & $21 \%$ & $16 \%$ & $22 \%$ & $8 \%$ & $9 \%$ & $13 \%$ & $11 \%$ \\
\hline Hesse & 0.034 & $10.1 \%$ & 0.191 & 0.192 & $20 \%$ & $21 \%$ & $23 \%$ & $9 \%$ & $7 \%$ & $8 \%$ & $11 \%$ \\
\hline Mecklenburg-W.Pomerania & 0.033 & $8.1 \%$ & 0.174 & 0.212 & $20 \%$ & $12 \%$ & $32 \%$ & $8 \%$ & $7 \%$ & $9 \%$ & $13 \%$ \\
\hline Lower Saxony & 0.025 & $5.7 \%$ & 0.171 & 0.168 & $25 \%$ & $25 \%$ & $23 \%$ & $6 \%$ & $6 \%$ & $4 \%$ & $12 \%$ \\
\hline North Rhine-Westphalia & 0.032 & $8.9 \%$ & 0.183 & 0.188 & $22 \%$ & $22 \%$ & $24 \%$ & $7 \%$ & $7 \%$ & $5 \%$ & $13 \%$ \\
\hline Rhineland-Palatinate & 0.033 & $8.2 \%$ & 0.191 & 0.170 & $19 \%$ & $26 \%$ & $21 \%$ & $6 \%$ & $5 \%$ & $10 \%$ & $13 \%$ \\
\hline Saarland & 0.035 & $9.2 \%$ & 0.196 & 0.178 & $21 \%$ & $24 \%$ & $24 \%$ & $9 \%$ & $3 \%$ & $5 \%$ & $13 \%$ \\
\hline Saxony & 0.034 & $9.4 \%$ & 0.181 & 0.162 & $20 \%$ & $12 \%$ & $32 \%$ & $12 \%$ & $4 \%$ & $6 \%$ & $15 \%$ \\
\hline Saxony-Anhalt & 0.036 & $10.4 \%$ & 0.188 & 0.180 & $19 \%$ & $14 \%$ & $29 \%$ & $11 \%$ & $6 \%$ & $7 \%$ & $13 \%$ \\
\hline Schleswig-Holstein & 0.024 & $5.5 \%$ & 0.169 & 0.174 & $18 \%$ & $22 \%$ & $30 \%$ & $9 \%$ & $5 \%$ & $5 \%$ & $11 \%$ \\
\hline Thuringia & 0.035 & $8.6 \%$ & 0.187 & 0.167 & $19 \%$ & $10 \%$ & $31 \%$ & $12 \%$ & $6 \%$ & $5 \%$ & $16 \%$ \\
\hline All & 0.032 & $8.8 \%$ & 0.185 & 0.181 & $21 \%$ & $20 \%$ & $25 \%$ & $8 \%$ & $6 \%$ & $7 \%$ & $13 \%$ \\
\hline
\end{tabular}


Table 3.12 GCSPI Decomposition 2006 (equal weights)

\begin{tabular}{|c|c|c|c|c|c|c|c|c|c|c|c|}
\hline \multirow[t]{2}{*}{ Bundeslaender } & \multirow[b]{2}{*}{$\overrightarrow{\tilde{v}}$} & \multirow{2}{*}{ 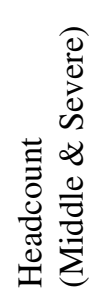 } & \multirow[b]{2}{*}{ 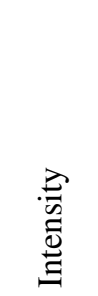 } & \multirow[b]{2}{*}{ 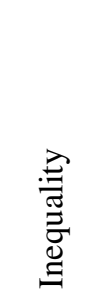 } & \multicolumn{7}{|c|}{ Contribution of Dimensions } \\
\hline & & & & & 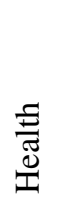 & 芯 & 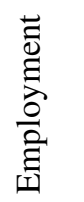 & 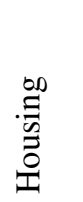 & 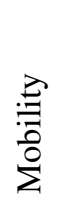 & 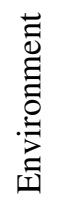 & $\begin{array}{l}\mathscr{\Xi} \\
\text { ठ } \\
\Xi\end{array}$ \\
\hline Baden-Württemberg & 0.034 & $8.8 \%$ & 0.195 & 0.163 & $20 \%$ & $26 \%$ & $21 \%$ & $8 \%$ & $5 \%$ & $10 \%$ & $9 \%$ \\
\hline Bavaria & 0.033 & $10.3 \%$ & 0.191 & 0.184 & $21 \%$ & $24 \%$ & $24 \%$ & $10 \%$ & $3 \%$ & $7 \%$ & $12 \%$ \\
\hline Berlin & 0.039 & $11.8 \%$ & 0.188 & 0.196 & $21 \%$ & $13 \%$ & $31 \%$ & $9 \%$ & $9 \%$ & $5 \%$ & $12 \%$ \\
\hline Brandenburg & 0.035 & $11.2 \%$ & 0.184 & 0.190 & $21 \%$ & $9 \%$ & $30 \%$ & $9 \%$ & $6 \%$ & $7 \%$ & $19 \%$ \\
\hline Bremen & 0.024 & $3.3 \%$ & 0.173 & 0.118 & $26 \%$ & $26 \%$ & $24 \%$ & $3 \%$ & $12 \%$ & $5 \%$ & $3 \%$ \\
\hline Hamburg & 0.026 & $9.0 \%$ & 0.172 & 0.179 & $22 \%$ & $16 \%$ & $22 \%$ & $10 \%$ & $13 \%$ & $7 \%$ & $11 \%$ \\
\hline Hesse & 0.033 & $9.6 \%$ & 0.193 & 0.171 & $24 \%$ & $19 \%$ & $25 \%$ & $7 \%$ & $7 \%$ & $7 \%$ & $11 \%$ \\
\hline Mecklenburg-W.Pomerania & 0.029 & $6.4 \%$ & 0.170 & 0.158 & $20 \%$ & $11 \%$ & $31 \%$ & $8 \%$ & $7 \%$ & $7 \%$ & $16 \%$ \\
\hline Lower Saxony & 0.029 & $7.2 \%$ & 0.176 & 0.187 & $22 \%$ & $23 \%$ & $25 \%$ & $6 \%$ & $5 \%$ & $4 \%$ & $15 \%$ \\
\hline North Rhine-Westphalia & 0.030 & $7.6 \%$ & 0.177 & 0.179 & $22 \%$ & $25 \%$ & $23 \%$ & $8 \%$ & $7 \%$ & $5 \%$ & $10 \%$ \\
\hline Rhineland-Palatinate & 0.038 & $12.3 \%$ & 0.207 & 0.158 & $20 \%$ & $21 \%$ & $21 \%$ & $7 \%$ & $4 \%$ & $12 \%$ & $15 \%$ \\
\hline Saarland & 0.044 & $13.7 \%$ & 0.207 & 0.205 & $16 \%$ & $17 \%$ & $29 \%$ & $15 \%$ & $2 \%$ & $4 \%$ & $17 \%$ \\
\hline Saxony & 0.038 & $11.6 \%$ & 0.188 & 0.187 & $22 \%$ & $9 \%$ & $31 \%$ & $10 \%$ & $4 \%$ & $8 \%$ & $17 \%$ \\
\hline Saxony-Anhalt & 0.034 & $9.6 \%$ & 0.185 & 0.165 & $23 \%$ & $11 \%$ & $32 \%$ & $10 \%$ & $6 \%$ & $3 \%$ & $15 \%$ \\
\hline Schleswig-Holstein & 0.022 & $4.0 \%$ & 0.167 & 0.140 & $28 \%$ & $20 \%$ & $26 \%$ & $6 \%$ & $4 \%$ & $5 \%$ & $12 \%$ \\
\hline Thuringia & 0.042 & $12.4 \%$ & 0.203 & 0.166 & $21 \%$ & $9 \%$ & $29 \%$ & $10 \%$ & $5 \%$ & $5 \%$ & $21 \%$ \\
\hline All & 0.033 & $9.1 \%$ & 0.186 & 0.178 & $22 \%$ & $20 \%$ & $25 \%$ & $8 \%$ & $5 \%$ & $6 \%$ & $13 \%$ \\
\hline
\end{tabular}

Table 3.13 GCSPI Decomposition 2008 (equal weights)

\begin{tabular}{|c|c|c|c|c|c|c|c|c|c|c|c|}
\hline \multirow[t]{2}{*}{ Bundeslaender } & \multirow[b]{2}{*}{$\underset{v}{\tilde{v}}$} & \multirow{2}{*}{ 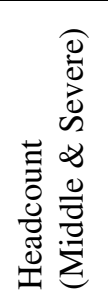 } & \multirow[b]{2}{*}{ 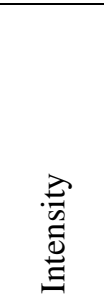 } & \multirow[b]{2}{*}{$\begin{array}{l}\stackrel{\Xi}{\Xi} \\
\stackrel{\Xi}{\Xi} \\
\stackrel{\Xi}{\Xi}\end{array}$} & \multicolumn{7}{|c|}{ Contribution of Dimensions } \\
\hline & & & & & 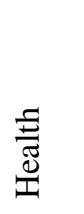 & 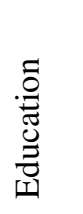 & 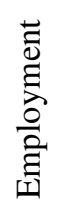 & $\begin{array}{l}\stackrel{0}{\Xi} \\
\stackrel{0}{0} \\
\stackrel{0}{0} \\
\stackrel{I}{I}\end{array}$ & 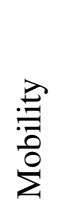 & 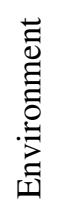 & $\begin{array}{l}\stackrel{\Xi}{\Xi} \\
\stackrel{0}{\Xi} \\
\Xi\end{array}$ \\
\hline Baden-Württemberg & 0.029 & $7.7 \%$ & 0.183 & 0.165 & $18 \%$ & $24 \%$ & $28 \%$ & $7 \%$ & $4 \%$ & $8 \%$ & $11 \%$ \\
\hline Bavaria & 0.028 & $7.1 \%$ & 0.179 & 0.159 & $20 \%$ & $22 \%$ & $28 \%$ & $9 \%$ & $3 \%$ & $7 \%$ & $11 \%$ \\
\hline Berlin & 0.040 & $13.2 \%$ & 0.199 & 0.178 & $17 \%$ & $12 \%$ & $33 \%$ & $9 \%$ & $7 \%$ & $7 \%$ & $15 \%$ \\
\hline Brandenburg & 0.037 & $11.4 \%$ & 0.195 & 0.187 & $17 \%$ & $13 \%$ & $32 \%$ & $8 \%$ & $5 \%$ & $3 \%$ & $23 \%$ \\
\hline Bremen & 0.026 & $4.2 \%$ & 0.177 & 0.102 & $14 \%$ & $22 \%$ & $24 \%$ & $10 \%$ & $18 \%$ & $9 \%$ & $3 \%$ \\
\hline Hamburg & 0.021 & $4.1 \%$ & 0.145 & 0.224 & $15 \%$ & $15 \%$ & $33 \%$ & $10 \%$ & $8 \%$ & $10 \%$ & $10 \%$ \\
\hline Hesse & 0.033 & $10.5 \%$ & 0.188 & 0.208 & $21 \%$ & $19 \%$ & $28 \%$ & $8 \%$ & $6 \%$ & $9 \%$ & $10 \%$ \\
\hline Mecklenburg-W.Pomerania & 0.033 & $9.1 \%$ & 0.186 & 0.161 & $17 \%$ & $10 \%$ & $36 \%$ & $7 \%$ & $3 \%$ & $4 \%$ & $22 \%$ \\
\hline Lower Saxony & 0.026 & $5.6 \%$ & 0.173 & 0.169 & $21 \%$ & $22 \%$ & $29 \%$ & $7 \%$ & $5 \%$ & $6 \%$ & $11 \%$ \\
\hline North Rhine-Westphalia & 0.031 & $8.8 \%$ & 0.185 & 0.177 & $20 \%$ & $20 \%$ & $28 \%$ & $7 \%$ & $6 \%$ & $8 \%$ & $12 \%$ \\
\hline Rhineland-Palatinate & 0.031 & $7.7 \%$ & 0.194 & 0.156 & $19 \%$ & $25 \%$ & $30 \%$ & $6 \%$ & $3 \%$ & $4 \%$ & $13 \%$ \\
\hline Saarland & 0.038 & $9.0 \%$ & 0.208 & 0.216 & $17 \%$ & $20 \%$ & $24 \%$ & $8 \%$ & $8 \%$ & $9 \%$ & $14 \%$ \\
\hline Saxony & 0.037 & $10.3 \%$ & 0.192 & 0.185 & $20 \%$ & $9 \%$ & $32 \%$ & $10 \%$ & $6 \%$ & $7 \%$ & $16 \%$ \\
\hline Saxony-Anhalt & 0.038 & $11.7 \%$ & 0.193 & 0.182 & $18 \%$ & $12 \%$ & $34 \%$ & $8 \%$ & $6 \%$ & $3 \%$ & $20 \%$ \\
\hline Schleswig-Holstein & 0.027 & $6.2 \%$ & 0.179 & 0.173 & $22 \%$ & $21 \%$ & $27 \%$ & $7 \%$ & $3 \%$ & $4 \%$ & $16 \%$ \\
\hline Thuringia & 0.034 & $10.5 \%$ & 0.190 & 0.150 & $20 \%$ & $9 \%$ & $34 \%$ & $9 \%$ & $3 \%$ & $4 \%$ & $21 \%$ \\
\hline All & 0.031 & $8.4 \%$ & 0.184 & 0.175 & $19 \%$ & $19 \%$ & $29 \%$ & $8 \%$ & $5 \%$ & $7 \%$ & $13 \%$ \\
\hline
\end{tabular}


Table 3.14 GCSPI Decomposition 2010 (equal weights)

\begin{tabular}{|c|c|c|c|c|c|c|c|c|c|c|c|}
\hline \multirow[t]{2}{*}{ Bundeslaender } & \multirow[b]{2}{*}{$\overrightarrow{\tilde{v}}$} & \multirow{2}{*}{ 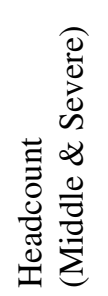 } & \multirow[b]{2}{*}{ 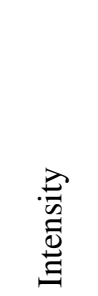 } & \multirow[b]{2}{*}{ 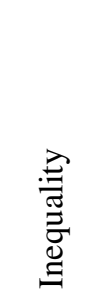 } & \multicolumn{7}{|c|}{ Contribution of Dimensions } \\
\hline & & & & & 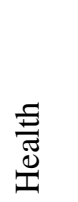 & 芯 & 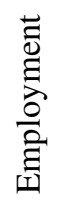 & 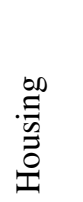 & 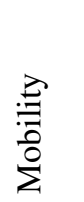 & 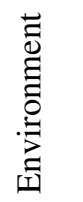 & $\begin{array}{l}\mathscr{\Xi} \\
\text { ठ } \\
\Xi\end{array}$ \\
\hline Baden-Württemberg & 0.028 & $7.5 \%$ & 0.176 & 0.165 & $22 \%$ & $24 \%$ & $27 \%$ & $7 \%$ & $4 \%$ & $7 \%$ & $9 \%$ \\
\hline Bavaria & 0.032 & $8.7 \%$ & 0.187 & 0.183 & $22 \%$ & $21 \%$ & $25 \%$ & $10 \%$ & $3 \%$ & $4 \%$ & $15 \%$ \\
\hline Berlin & 0.048 & $13.4 \%$ & 0.208 & 0.210 & $24 \%$ & $13 \%$ & $25 \%$ & $9 \%$ & $6 \%$ & $9 \%$ & $14 \%$ \\
\hline Brandenburg & 0.031 & $8.8 \%$ & 0.174 & 0.206 & $18 \%$ & $10 \%$ & $33 \%$ & $8 \%$ & $6 \%$ & $4 \%$ & $21 \%$ \\
\hline Bremen & 0.028 & $5.8 \%$ & 0.185 & 0.102 & $24 \%$ & $17 \%$ & $23 \%$ & $2 \%$ & $17 \%$ & $15 \%$ & $3 \%$ \\
\hline Hamburg & 0.018 & $3.6 \%$ & 0.134 & 0.219 & $17 \%$ & $16 \%$ & $36 \%$ & $6 \%$ & $7 \%$ & $10 \%$ & $8 \%$ \\
\hline Hesse & 0.030 & $7.9 \%$ & 0.185 & 0.181 & $22 \%$ & $17 \%$ & $28 \%$ & $8 \%$ & $5 \%$ & $9 \%$ & $11 \%$ \\
\hline Mecklenburg-W.Pomerania & 0.032 & $8.0 \%$ & 0.186 & 0.125 & $24 \%$ & $7 \%$ & $31 \%$ & $7 \%$ & $5 \%$ & $5 \%$ & $22 \%$ \\
\hline Lower Saxony & 0.027 & $6.9 \%$ & 0.180 & 0.193 & $20 \%$ & $20 \%$ & $28 \%$ & $6 \%$ & $4 \%$ & $6 \%$ & $15 \%$ \\
\hline North Rhine-Westphalia & 0.029 & $7.8 \%$ & 0.177 & 0.182 & $22 \%$ & $21 \%$ & $27 \%$ & $6 \%$ & $7 \%$ & $5 \%$ & $11 \%$ \\
\hline Rhineland-Palatinate & 0.035 & $11.2 \%$ & 0.188 & 0.209 & $17 \%$ & $24 \%$ & $29 \%$ & $5 \%$ & $4 \%$ & $3 \%$ & $18 \%$ \\
\hline Saarland & 0.040 & $16.6 \%$ & 0.214 & 0.181 & $13 \%$ & $18 \%$ & $27 \%$ & $12 \%$ & $5 \%$ & $6 \%$ & $19 \%$ \\
\hline Saxony & 0.038 & $11.3 \%$ & 0.191 & 0.195 & $21 \%$ & $8 \%$ & $31 \%$ & $11 \%$ & $7 \%$ & $6 \%$ & $15 \%$ \\
\hline Saxony-Anhalt & 0.036 & $9.7 \%$ & 0.183 & 0.231 & $20 \%$ & $13 \%$ & $32 \%$ & $9 \%$ & $4 \%$ & $4 \%$ & $17 \%$ \\
\hline Schleswig-Holstein & 0.023 & $5.4 \%$ & 0.166 & 0.147 & $27 \%$ & $20 \%$ & $26 \%$ & $9 \%$ & $4 \%$ & $5 \%$ & $9 \%$ \\
\hline Thuringia & 0.037 & $12.4 \%$ & 0.198 & 0.164 & $20 \%$ & $9 \%$ & $33 \%$ & $9 \%$ & $3 \%$ & $5 \%$ & $21 \%$ \\
\hline All & 0.031 & $8.6 \%$ & 0.182 & 0.186 & $21 \%$ & $18 \%$ & $28 \%$ & $8 \%$ & $5 \%$ & $6 \%$ & $14 \%$ \\
\hline
\end{tabular}

Table 3.15 SCSPI Decomposition 2002

\begin{tabular}{|c|c|c|c|c|c|c|c|c|}
\hline \multirow[t]{2}{*}{ Bundeslaender } & \multirow[b]{2}{*}{$\overrightarrow{\tilde{n}}$} & \multirow{2}{*}{ 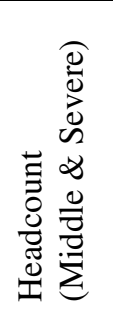 } & \multirow[b]{2}{*}{ 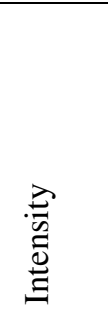 } & \multirow[b]{2}{*}{ 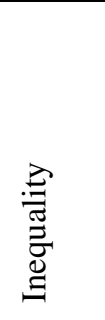 } & \multicolumn{4}{|c|}{ Contribution of Dimensions } \\
\hline & & & & & 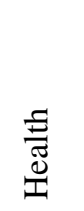 & 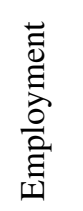 & 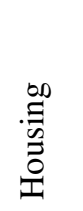 & 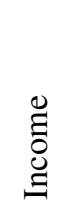 \\
\hline Baden-Württemberg & 0.028 & $6.6 \%$ & 0.345 & 0.090 & $22 \%$ & $29 \%$ & $13 \%$ & $35 \%$ \\
\hline Bavaria & 0.033 & $6.3 \%$ & 0.366 & 0.130 & $21 \%$ & $27 \%$ & $21 \%$ & $31 \%$ \\
\hline Berlin & 0.055 & $11.6 \%$ & 0.386 & 0.132 & $20 \%$ & $28 \%$ & $15 \%$ & $37 \%$ \\
\hline Brandenburg & 0.052 & $11.3 \%$ & 0.346 & 0.104 & $15 \%$ & $26 \%$ & $19 \%$ & $40 \%$ \\
\hline Bremen & 0.038 & $7.2 \%$ & 0.368 & 0.125 & $21 \%$ & $29 \%$ & $15 \%$ & $36 \%$ \\
\hline Hamburg & 0.050 & $12.9 \%$ & 0.388 & 0.090 & $24 \%$ & $29 \%$ & $15 \%$ & $32 \%$ \\
\hline Hesse & 0.038 & $6.7 \%$ & 0.363 & 0.150 & $21 \%$ & $29 \%$ & $19 \%$ & $31 \%$ \\
\hline Mecklenburg-W.Pomerania & 0.039 & $7.3 \%$ & 0.347 & 0.126 & $18 \%$ & $31 \%$ & $10 \%$ & $41 \%$ \\
\hline Lower Saxony & 0.046 & $10.3 \%$ & 0.371 & 0.112 & $25 \%$ & $29 \%$ & $11 \%$ & $36 \%$ \\
\hline North Rhine-Westphalia & 0.028 & $4.7 \%$ & 0.339 & 0.137 & $23 \%$ & $30 \%$ & $16 \%$ & $32 \%$ \\
\hline Rhineland-Palatinate & 0.023 & $4.6 \%$ & 0.344 & 0.111 & $30 \%$ & $23 \%$ & $13 \%$ & $34 \%$ \\
\hline Saarland & 0.024 & $3.3 \%$ & 0.326 & 0.163 & $20 \%$ & $36 \%$ & $15 \%$ & $29 \%$ \\
\hline Saxony & 0.038 & $9.7 \%$ & 0.351 & 0.082 & $11 \%$ & $29 \%$ & $14 \%$ & $46 \%$ \\
\hline Saxony-Anhalt & 0.041 & $9.1 \%$ & 0.349 & 0.099 & $19 \%$ & $23 \%$ & $15 \%$ & $43 \%$ \\
\hline Schleswig-Holstein & 0.038 & $8.3 \%$ & 0.404 & 0.116 & $26 \%$ & $32 \%$ & $14 \%$ & $28 \%$ \\
\hline Thuringia & 0.052 & $12.4 \%$ & 0.377 & 0.104 & $17 \%$ & $36 \%$ & $8 \%$ & $38 \%$ \\
\hline All & 0.035 & $7.3 \%$ & 0.358 & 0.119 & $21 \%$ & $29 \%$ & $15 \%$ & $35 \%$ \\
\hline
\end{tabular}


Table 3.16 SCSPI Decomposition 2004

\begin{tabular}{|c|c|c|c|c|c|c|c|c|}
\hline \multirow[t]{2}{*}{ Bundeslaender } & \multirow[b]{2}{*}{$\overrightarrow{\tilde{n}}$} & \multirow{2}{*}{ 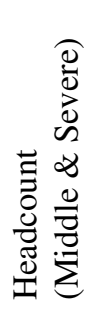 } & \multirow[b]{2}{*}{ 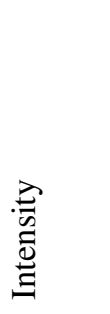 } & \multirow[b]{2}{*}{ 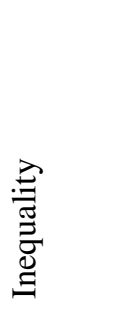 } & \multicolumn{4}{|c|}{ Contribution of Dimensions } \\
\hline & & & & & 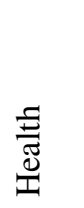 & 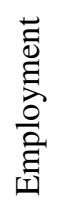 & 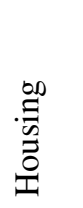 & $\begin{array}{l}\mathscr{\Xi} \\
\text { ठ } \\
\Xi\end{array}$ \\
\hline Baden-Württemberg & 0.040 & $7.3 \%$ & 0.356 & 0.136 & $26 \%$ & $25 \%$ & $15 \%$ & $35 \%$ \\
\hline Bavaria & 0.044 & $8.6 \%$ & 0.378 & 0.135 & $25 \%$ & $25 \%$ & $15 \%$ & $35 \%$ \\
\hline Berlin & 0.048 & $12.4 \%$ & 0.387 & 0.091 & $22 \%$ & $28 \%$ & $6 \%$ & $43 \%$ \\
\hline Brandenburg & 0.059 & $13.3 \%$ & 0.358 & 0.102 & $19 \%$ & $29 \%$ & $3 \%$ & $48 \%$ \\
\hline Bremen & 0.040 & $9.4 \%$ & 0.410 & 0.105 & $17 \%$ & $35 \%$ & $2 \%$ & $46 \%$ \\
\hline Hamburg & 0.047 & $6.7 \%$ & 0.345 & 0.147 & $20 \%$ & $24 \%$ & $21 \%$ & $36 \%$ \\
\hline Hesse & 0.033 & $7.5 \%$ & 0.338 & 0.089 & $19 \%$ & $35 \%$ & $9 \%$ & $37 \%$ \\
\hline Mecklenburg-W.Pomerania & 0.045 & $11.3 \%$ & 0.341 & 0.076 & $15 \%$ & $26 \%$ & $5 \%$ & $54 \%$ \\
\hline Lower Saxony & 0.042 & $9.3 \%$ & 0.363 & 0.108 & $25 \%$ & $30 \%$ & $9 \%$ & $36 \%$ \\
\hline North Rhine-Westphalia & 0.036 & $6.3 \%$ & 0.351 & 0.143 & $25 \%$ & $26 \%$ & $16 \%$ & $33 \%$ \\
\hline Rhineland-Palatinate & 0.024 & $4.2 \%$ & 0.310 & 0.094 & $24 \%$ & $32 \%$ & $9 \%$ & $35 \%$ \\
\hline Saarland & 0.016 & $3.1 \%$ & 0.306 & 0.065 & $31 \%$ & $23 \%$ & $2 \%$ & $44 \%$ \\
\hline Saxony & 0.060 & $12.1 \%$ & 0.392 & 0.135 & $25 \%$ & $29 \%$ & $8 \%$ & $38 \%$ \\
\hline Saxony-Anhalt & 0.065 & $13.3 \%$ & 0.402 & 0.137 & $22 \%$ & $22 \%$ & $14 \%$ & $42 \%$ \\
\hline Schleswig-Holstein & 0.034 & $6.7 \%$ & 0.366 & 0.122 & $24 \%$ & $29 \%$ & $9 \%$ & $38 \%$ \\
\hline Thuringia & 0.052 & $11.0 \%$ & 0.344 & 0.105 & $15 \%$ & $35 \%$ & $10 \%$ & $41 \%$ \\
\hline All & 0.041 & $8.2 \%$ & 0.360 & 0.125 & $23 \%$ & $28 \%$ & $12 \%$ & $37 \%$ \\
\hline
\end{tabular}

Table 3.17 SCSPI Decomposition 2006

\begin{tabular}{|c|c|c|c|c|c|c|c|c|}
\hline \multirow[t]{2}{*}{ Bundeslaender } & \multirow[b]{2}{*}{$\overrightarrow{\tilde{v}}$} & \multirow{2}{*}{ 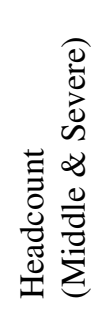 } & \multirow[b]{2}{*}{ 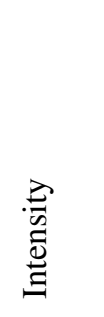 } & \multirow[b]{2}{*}{ 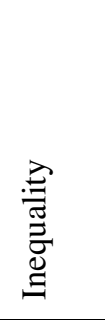 } & \multicolumn{4}{|c|}{ Contribution of Dimensions } \\
\hline & & & & & $\begin{array}{l}\stackrel{\Xi}{\Xi} \\
\stackrel{\Xi}{\mathbb{I}}\end{array}$ & 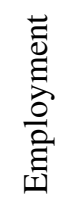 & 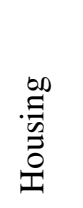 & $\begin{array}{l}\mathscr{\Xi} \\
\Xi \\
\Xi\end{array}$ \\
\hline Baden-Württemberg & 0.036 & $6.8 \%$ & 0.344 & 0.128 & $23 \%$ & $26 \%$ & $14 \%$ & $37 \%$ \\
\hline Bavaria & 0.041 & $8.1 \%$ & 0.355 & 0.123 & $23 \%$ & $26 \%$ & $15 \%$ & $36 \%$ \\
\hline Berlin & 0.070 & $14.0 \%$ & 0.403 & 0.134 & $25 \%$ & $30 \%$ & $10 \%$ & $35 \%$ \\
\hline Brandenburg & 0.045 & $11.3 \%$ & 0.342 & 0.077 & $20 \%$ & $22 \%$ & $12 \%$ & $46 \%$ \\
\hline Bremen & 0.049 & $12.8 \%$ & 0.455 & 0.081 & $32 \%$ & $38 \%$ & $0 \%$ & $30 \%$ \\
\hline Hamburg & 0.032 & $4.5 \%$ & 0.390 & 0.180 & $17 \%$ & $23 \%$ & $21 \%$ & $39 \%$ \\
\hline Hesse & 0.042 & $8.3 \%$ & 0.355 & 0.128 & $21 \%$ & $33 \%$ & $14 \%$ & $32 \%$ \\
\hline Mecklenburg-W.Pomerania & 0.041 & $9.2 \%$ & 0.341 & 0.094 & $20 \%$ & $15 \%$ & $12 \%$ & $52 \%$ \\
\hline Lower Saxony & 0.048 & $11.5 \%$ & 0.379 & 0.103 & $24 \%$ & $31 \%$ & $12 \%$ & $33 \%$ \\
\hline North Rhine-Westphalia & 0.032 & $6.0 \%$ & 0.343 & 0.120 & $23 \%$ & $29 \%$ & $14 \%$ & $34 \%$ \\
\hline Rhineland-Palatinate & 0.057 & $11.9 \%$ & 0.442 & 0.121 & $20 \%$ & $28 \%$ & $18 \%$ & $35 \%$ \\
\hline Saarland & 0.039 & $7.6 \%$ & 0.333 & 0.102 & $25 \%$ & $35 \%$ & $4 \%$ & $36 \%$ \\
\hline Saxony & 0.041 & $8.2 \%$ & 0.360 & 0.121 & $17 \%$ & $29 \%$ & $10 \%$ & $44 \%$ \\
\hline Saxony-Anhalt & 0.035 & $5.6 \%$ & 0.300 & 0.078 & $20 \%$ & $26 \%$ & $6 \%$ & $48 \%$ \\
\hline Schleswig-Holstein & 0.032 & $5.8 \%$ & 0.330 & 0.117 & $22 \%$ & $28 \%$ & $13 \%$ & $37 \%$ \\
\hline Thuringia & 0.062 & $12.9 \%$ & 0.380 & 0.127 & $17 \%$ & $28 \%$ & $12 \%$ & $42 \%$ \\
\hline All & 0.041 & $8.3 \%$ & 0.358 & 0.122 & $22 \%$ & $28 \%$ & $13 \%$ & $36 \%$ \\
\hline
\end{tabular}


Table 3.18 SCSPI Decomposition 2008

\begin{tabular}{|c|c|c|c|c|c|c|c|c|}
\hline \multirow[t]{2}{*}{ Bundeslaender } & \multirow[b]{2}{*}{$\underset{\tilde{n}}{\bar{n}}$} & \multirow{2}{*}{ 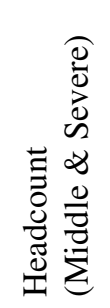 } & \multirow[b]{2}{*}{ 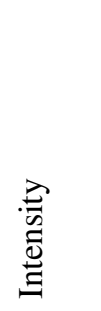 } & \multirow[b]{2}{*}{ 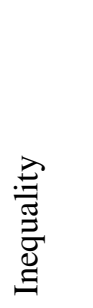 } & \multicolumn{4}{|c|}{ Contribution of Dimensions } \\
\hline & & & & & 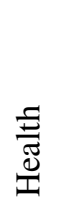 & 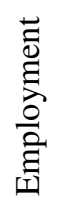 & 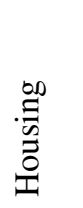 & $\begin{array}{l}\mathscr{\Xi} \\
\text { ठ } \\
\Xi\end{array}$ \\
\hline Baden-Württemberg & 0.033 & $7.2 \%$ & 0.344 & 0.098 & $21 \%$ & $29 \%$ & $8 \%$ & $42 \%$ \\
\hline Bavaria & 0.042 & $9.3 \%$ & 0.373 & 0.113 & $24 \%$ & $27 \%$ & $14 \%$ & $35 \%$ \\
\hline Berlin & 0.055 & $11.1 \%$ & 0.377 & 0.135 & $25 \%$ & $26 \%$ & $12 \%$ & $37 \%$ \\
\hline Brandenburg & 0.041 & $10.3 \%$ & 0.357 & 0.085 & $19 \%$ & $21 \%$ & $12 \%$ & $49 \%$ \\
\hline Bremen & 0.017 & $2.3 \%$ & 0.278 & 0.041 & $17 \%$ & $55 \%$ & $0 \%$ & $28 \%$ \\
\hline Hamburg & 0.030 & $7.2 \%$ & 0.386 & 0.099 & $18 \%$ & $35 \%$ & $11 \%$ & $35 \%$ \\
\hline Hesse & 0.039 & $8.4 \%$ & 0.347 & 0.101 & $23 \%$ & $28 \%$ & $13 \%$ & $36 \%$ \\
\hline Mecklenburg-W.Pomerania & 0.030 & $5.3 \%$ & 0.306 & 0.079 & $17 \%$ & $25 \%$ & $1 \%$ & $57 \%$ \\
\hline Lower Saxony & 0.037 & $7.8 \%$ & 0.370 & 0.121 & $23 \%$ & $28 \%$ & $14 \%$ & $34 \%$ \\
\hline North Rhine-Westphalia & 0.034 & $6.5 \%$ & 0.355 & 0.128 & $26 \%$ & $26 \%$ & $15 \%$ & $32 \%$ \\
\hline Rhineland-Palatinate & 0.029 & $6.3 \%$ & 0.382 & 0.126 & $22 \%$ & $26 \%$ & $19 \%$ & $34 \%$ \\
\hline Saarland & 0.028 & $6.0 \%$ & 0.329 & 0.085 & $21 \%$ & $20 \%$ & $12 \%$ & $46 \%$ \\
\hline Saxony & 0.041 & $9.9 \%$ & 0.359 & 0.092 & $17 \%$ & $30 \%$ & $8 \%$ & $45 \%$ \\
\hline Saxony-Anhalt & 0.057 & $14.2 \%$ & 0.400 & 0.106 & $18 \%$ & $29 \%$ & $7 \%$ & $46 \%$ \\
\hline Schleswig-Holstein & 0.030 & $7.0 \%$ & 0.345 & 0.090 & $25 \%$ & $28 \%$ & $3 \%$ & $45 \%$ \\
\hline Thuringia & 0.054 & $10.2 \%$ & 0.363 & 0.141 & $19 \%$ & $28 \%$ & $11 \%$ & $42 \%$ \\
\hline All & 0.038 & $8.1 \%$ & 0.359 & 0.114 & $23 \%$ & $28 \%$ & $12 \%$ & $38 \%$ \\
\hline
\end{tabular}

Table 3.19 SCSPI Decomposition 2010

\begin{tabular}{|c|c|c|c|c|c|c|c|c|}
\hline \multirow[t]{2}{*}{ Bundeslaender } & \multirow[b]{2}{*}{$\overrightarrow{\tilde{v}}$} & \multirow{2}{*}{ 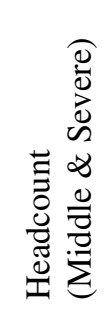 } & \multirow[b]{2}{*}{ 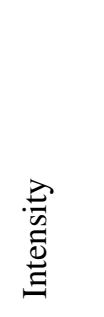 } & \multirow[b]{2}{*}{ 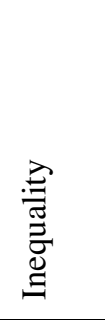 } & \multicolumn{4}{|c|}{ Contribution of Dimensions } \\
\hline & & & & & 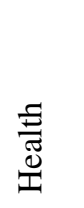 & 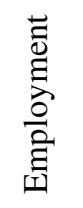 & 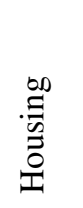 & $\begin{array}{l}\mathscr{\Xi} \\
\Xi \\
\Xi\end{array}$ \\
\hline Baden-Württemberg & 0.043 & $\overline{10.0 \%}$ & 0.364 & 0.106 & $23 \%$ & $28 \%$ & $14 \%$ & $36 \%$ \\
\hline Bavaria & 0.043 & $9.4 \%$ & 0.367 & 0.112 & $18 \%$ & $30 \%$ & $17 \%$ & $35 \%$ \\
\hline Berlin & 0.068 & $12.8 \%$ & 0.428 & 0.149 & $25 \%$ & $25 \%$ & $18 \%$ & $32 \%$ \\
\hline Brandenburg & 0.073 & $17.6 \%$ & 0.419 & 0.103 & $28 \%$ & $22 \%$ & $16 \%$ & $34 \%$ \\
\hline Bremen & 0.021 & $1.9 \%$ & 0.268 & 0.029 & $33 \%$ & $29 \%$ & $2 \%$ & $35 \%$ \\
\hline Hamburg & 0.030 & $6.7 \%$ & 0.310 & 0.061 & $14 \%$ & $30 \%$ & $11 \%$ & $44 \%$ \\
\hline Hesse & 0.036 & $6.9 \%$ & 0.365 & 0.127 & $23 \%$ & $30 \%$ & $16 \%$ & $31 \%$ \\
\hline Mecklenburg-W.Pomerania & 0.050 & $8.5 \%$ & 0.373 & 0.141 & $27 \%$ & $29 \%$ & $5 \%$ & $39 \%$ \\
\hline Lower Saxony & 0.038 & $9.7 \%$ & 0.358 & 0.091 & $23 \%$ & $24 \%$ & $15 \%$ & $38 \%$ \\
\hline North Rhine-Westphalia & 0.037 & $8.1 \%$ & 0.381 & 0.117 & $24 \%$ & $28 \%$ & $17 \%$ & $30 \%$ \\
\hline Rhineland-Palatinate & 0.028 & $6.0 \%$ & 0.371 & 0.117 & $29 \%$ & $29 \%$ & $18 \%$ & $24 \%$ \\
\hline Saarland & 0.047 & $12.4 \%$ & 0.371 & 0.084 & $10 \%$ & $20 \%$ & $22 \%$ & $48 \%$ \\
\hline Saxony & 0.045 & $7.8 \%$ & 0.341 & 0.141 & $22 \%$ & $23 \%$ & $12 \%$ & $43 \%$ \\
\hline Saxony-Anhalt & 0.053 & $8.5 \%$ & 0.376 & 0.166 & $29 \%$ & $25 \%$ & $13 \%$ & $33 \%$ \\
\hline Schleswig-Holstein & 0.044 & $5.4 \%$ & 0.393 & 0.206 & $26 \%$ & $30 \%$ & $15 \%$ & $30 \%$ \\
\hline Thuringia & 0.036 & $7.1 \%$ & 0.312 & 0.082 & $13 \%$ & $32 \%$ & $10 \%$ & $46 \%$ \\
\hline All & 0.042 & $8.9 \%$ & 0.369 & 0.121 & $23 \%$ & $27 \%$ & $15 \%$ & $35 \%$ \\
\hline
\end{tabular}

